Aus der Abteilung Medizinische Mikrobiologie

(Prof. Dr. med. U. Groß)

im Zentrum Hygiene und Humangenetik

der Medizinischen Fakultät der Universität Göttingen

\title{
Epidemiologie und Empfindlichkeit von Pilzisolaten gegenüber sechs Antimykotika aus primär sterilen Materialien in Deutschland
}

\author{
Inaugural-Dissertation \\ zur Erlangung des Doktorgrades \\ der Medizinischen Fakultät \\ der Georg-August-Universität zu Göttingen
}

\author{
vorgelegt von \\ Luisa Kunz \\ aus \\ Henstedt-Ulzburg
}

Göttingen 2012 
Dekan: Prof. Dr. med. C. Frömmel

1. Berichterstatterin: Prof. Dr. med. M. Borg-von Zepelin

2. Berichterstatter: Priv.-Doz. Dr. med. Zietz

3. Berichterstatterin: Prof. Dr. rer. nat. Virsik-Köpp

Tag der mündlichen Prüfung: 20. August 2012 


\section{Inhaltsverzeichnis}

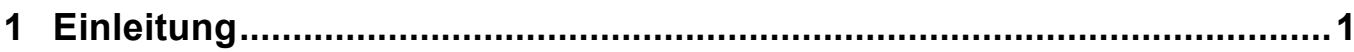

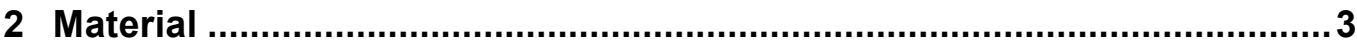

2.1 Herkunft der Verbrauchsmaterialien und Chemikalien ........................... 3

2.2 Herkunft der Antimykotika ........................................................... 4

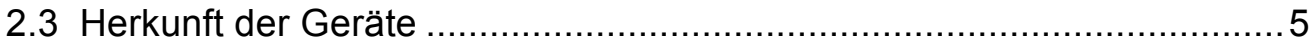

2.4 Herkunft der Referenzstämme ...................................................

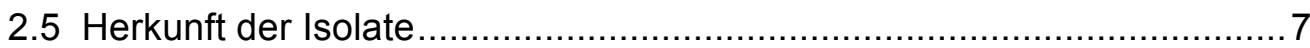

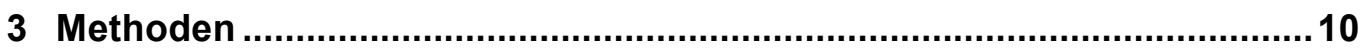

3.1 Puffer und Kulturmedien .................................................................. 10

3.1.1 Physiologische Kochsalzlösung .........................................10

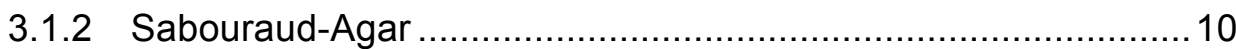

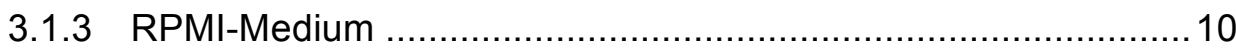

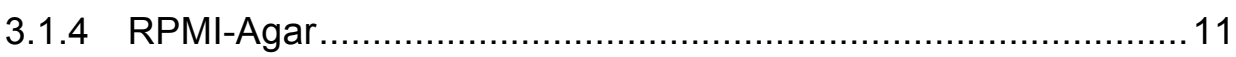

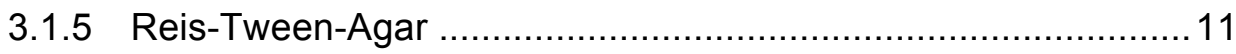

3.2 Differenzierung der Hefen ............................................................ 12

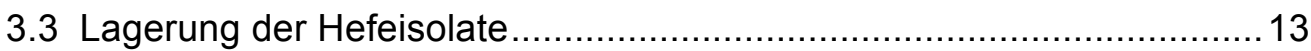

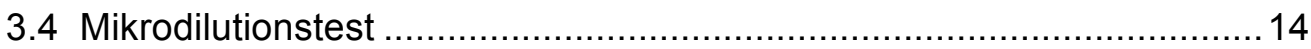

3.4.1 Präparation des Inokulums.................................................... 14

3.4.2 Kontrolle der Einsaat ............................................................. 14

3.4.3 Präparation der Antimykotika-Stammlösungen ........................ 15

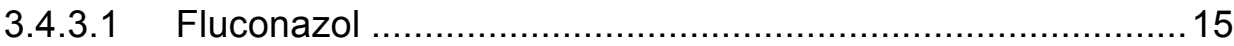

3.4.3.2 Itraconazol, Voriconazol und Amphotericin B .....................15

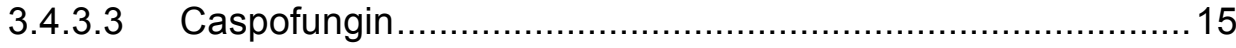

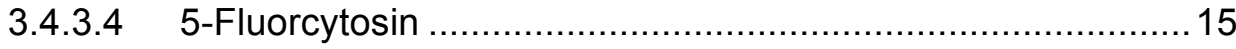

3.4.4 Präparation der Verdünnungsreihen .................................... 16

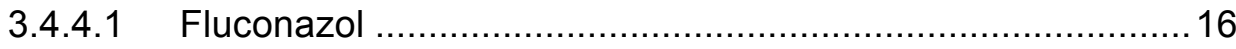

3.4.4.2 Itraconazol, Voriconazol und Amphotericin B ..................... 18

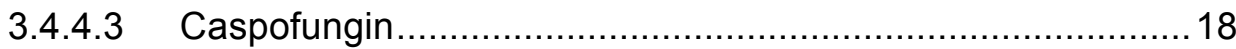




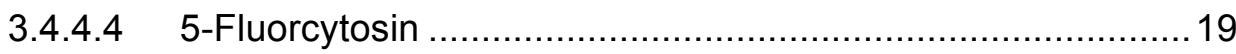

3.4.5 Beschickung der Mikrotestplatten ......................................... 19

3.4.5.1 Antimykotika-Suspensionen und RPMI-Medium ...................19

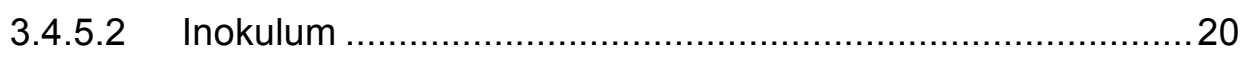

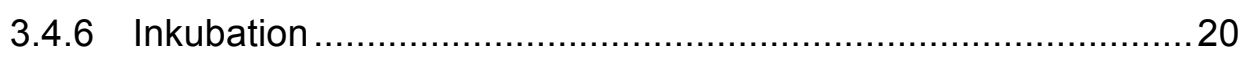

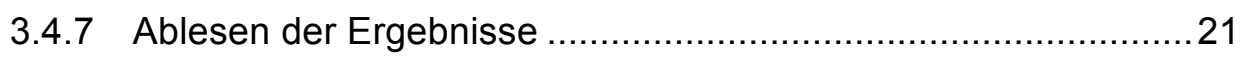

3.4.7.1 Makroskopisches Ablesen ............................................. 21

3.4.7.2 Photometrisches Ablesen .................................................21

3.4.8 Bewertung der Ergebnisse ..................................................22

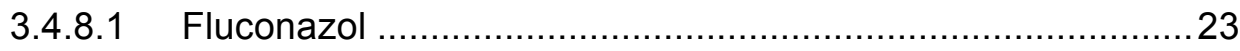

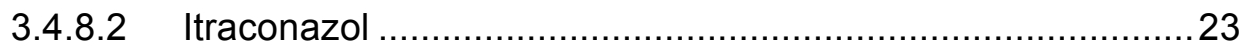

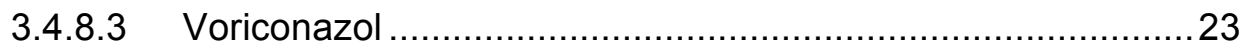

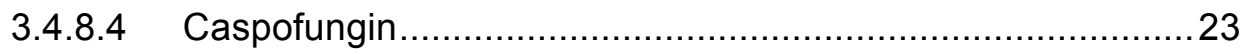

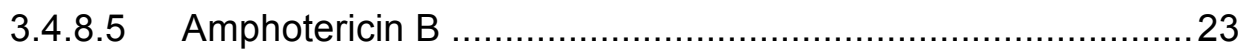

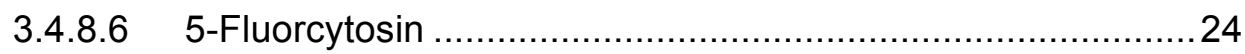

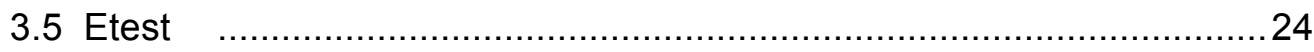

3.5.1 Präparation und Auftragen des Inokulums ...............................25

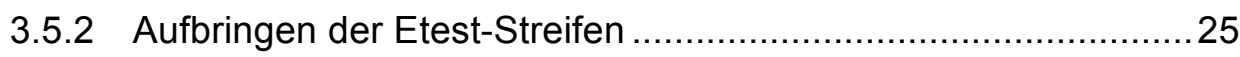

3.5.3 Inkubation und Ablesen der Ergebnisse..................................26

3.5.4 Bewertung der Ergebnisse ............................................26

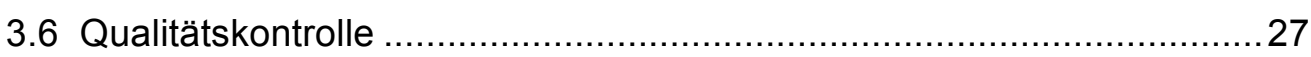

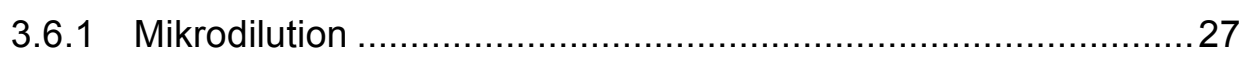

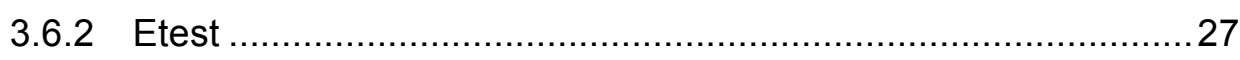

3.7 Korrelation der Testmethoden Mikrodilution und Etest ........................28

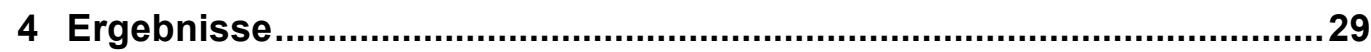

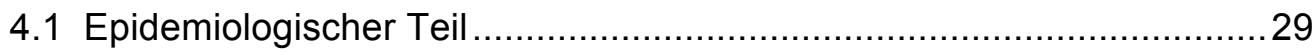

4.1.1 Teilnehmende Institutionen ...........................................29

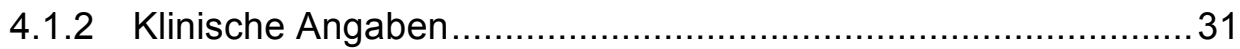

4.1.3 Speziesverteilung der Isolate in allen Materialien .....................31

4.1.4 Speziesverteilung in Bezug auf Geschlecht und Alter...............33

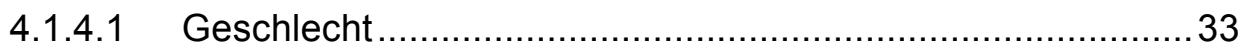




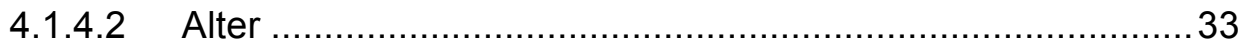

4.1.5 Speziesverteilung der Isolate in den einzelnen Materialien .......34

4.1.5.1 Speziesverteilung in den Proben aus Blutkulturen ................35

4.1.5.2 Speziesverteilung in den Proben aus Liquor ........................ 35

4.1.5.3 Speziesverteilung in den Proben aus ZVK-Spitzen ...............35

4.1.5.4 Speziesverteilung in den Proben aus Brust- und Bauchraum35

4.1.5.5 Speziesverteilung in den Proben aus Bronchiallavagen.........35

4.1.5.6 Speziesverteilung in den Proben übriger Körperregionen ..... 36

4.2 Ergebnisse des Mikrodilutionstests .................................................. 37

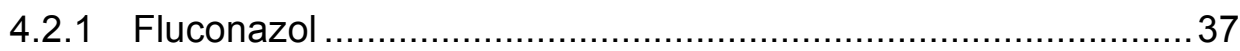

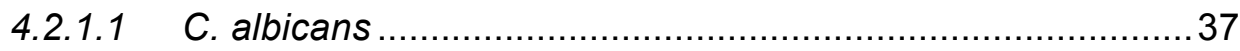

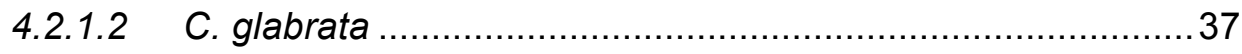

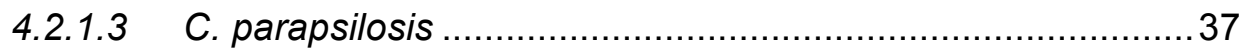

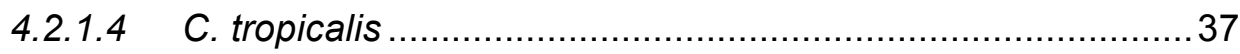

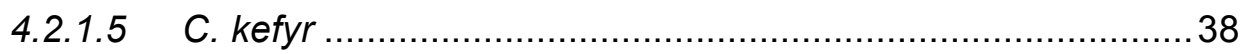

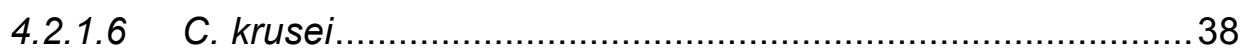

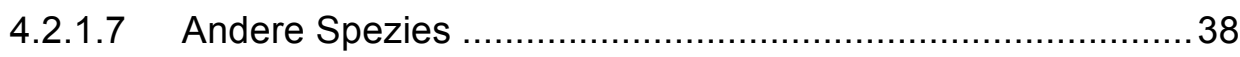

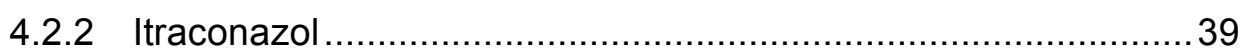

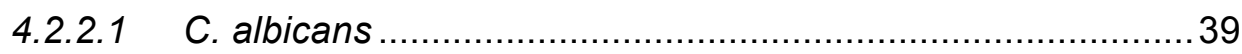

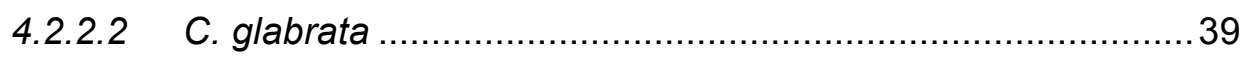

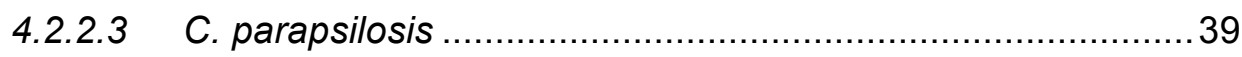

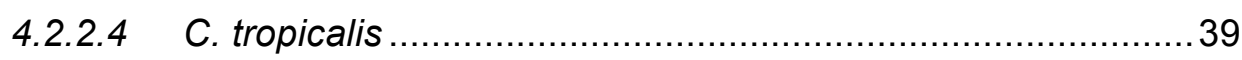

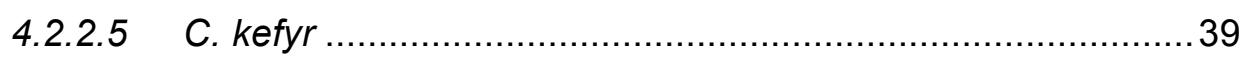

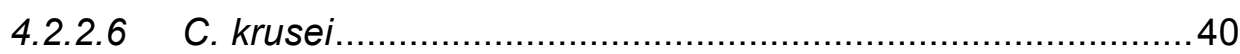

4.2.2.7 Andere Spezies ............................................................ 40

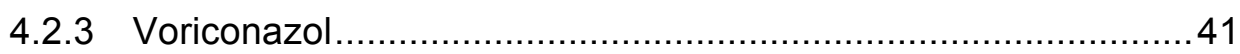

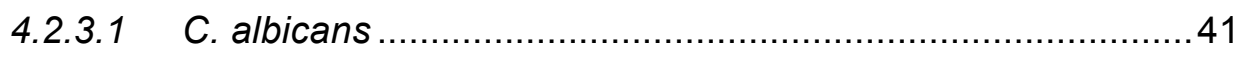

4.2.3.2 C. glabrata .................................................................... 41

4.2.3.3 C. parapsilosis ............................................................. 41

4.2.3.4 C. tropicalis .............................................................. 41

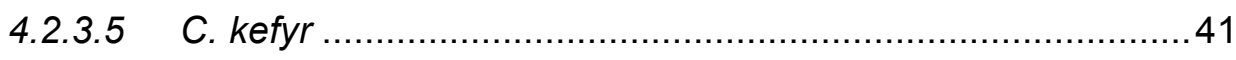




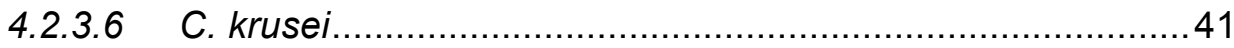

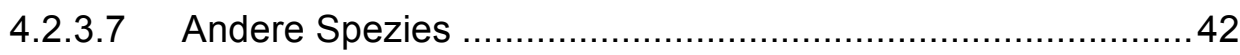

4.2.4 Kreuzresistenzen der Azole ...............................................42

4.2.5 Besonderheiten der Spezies C. glabrata gegenüber den getesteten Antimykotika ................................................... 44

4.2.6 Caspofungin ............................................................... 45

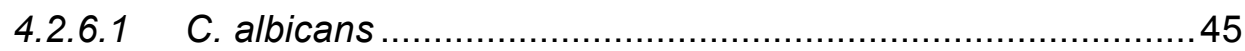

4.2.6.2 C. glabrata ................................................................ 46

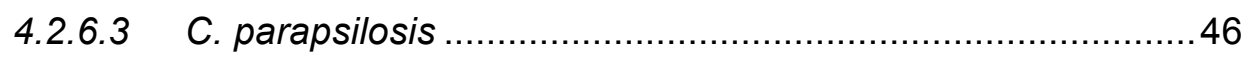

4.2.6.4 C. tropicalis ............................................................. 46

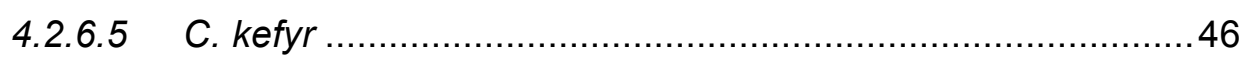

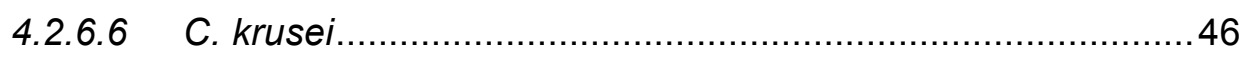

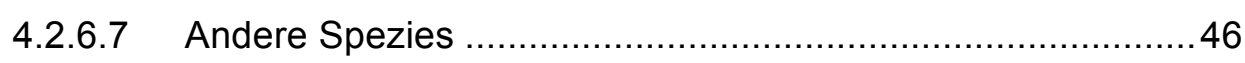

4.2.7 Amphotericin B ........................................................ 47

4.2.7.1 C. albicans .............................................................. 47

4.2.7.2 C. glabrata .................................................................. 47

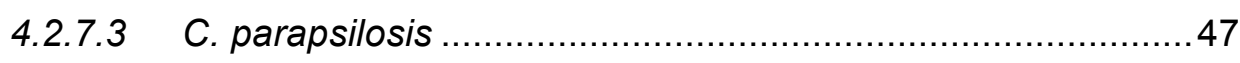

4.2.7.4 C. tropicalis .............................................................. 48

4.2.7.5 C. kefyr .................................................................. 48

4.2.7.6 C. krusei................................................................. 48

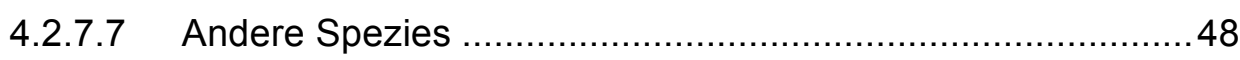

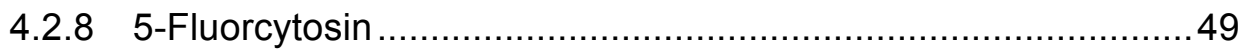

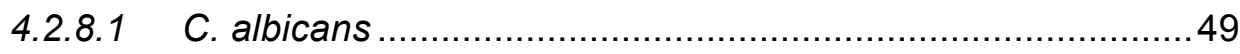

4.2.8.2 C. glabrata ............................................................... 49

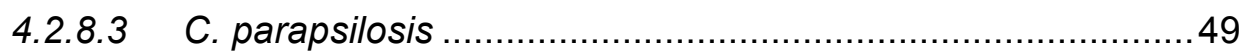

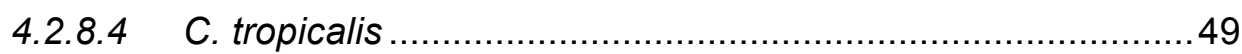

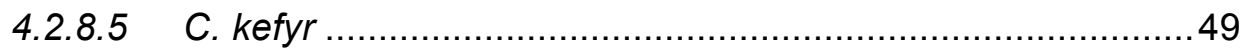

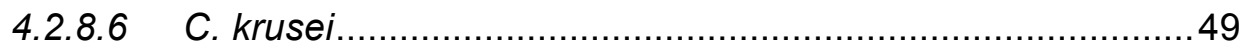

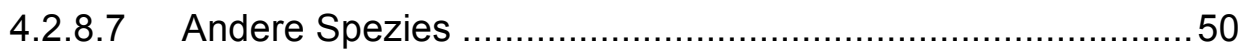

4.2.9 Zusammenfassung aller Mikrodilutions-Ergebnisse .................50

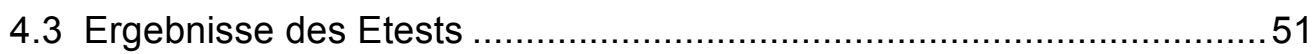




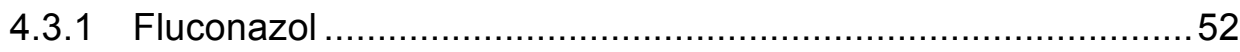

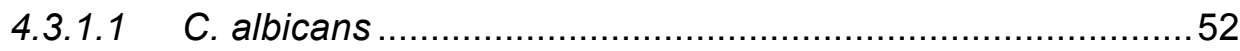

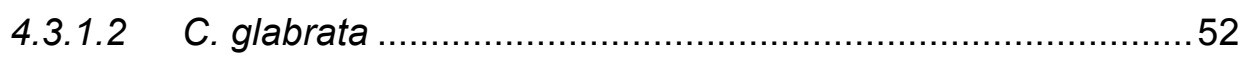

4.3.1.3 C. parapsilosis ........................................................ 52

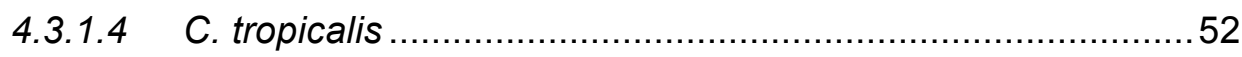

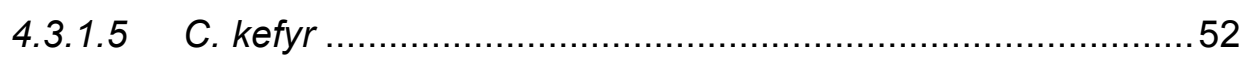

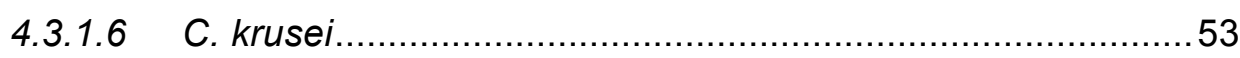

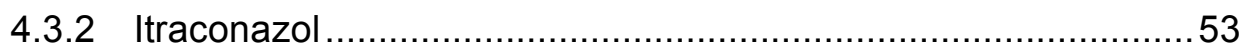

4.3.2.1 C. albicans .................................................................. 53

4.3.2.2 C. glabrata .............................................................. 54

4.3.2.3 C. parapsilosis ........................................................... 54

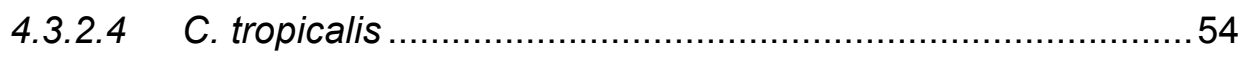

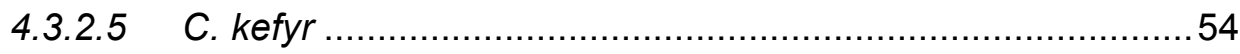

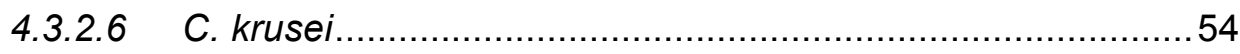

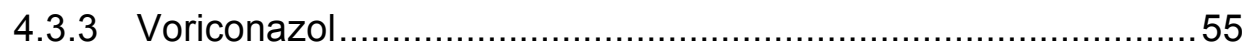

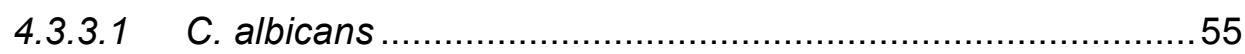

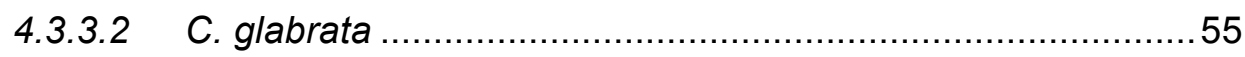

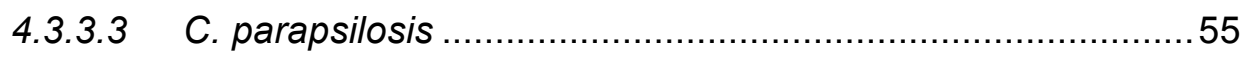

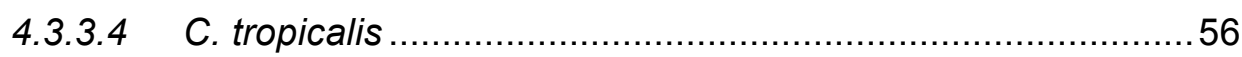

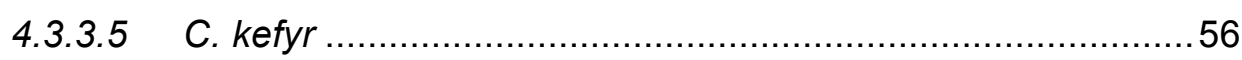

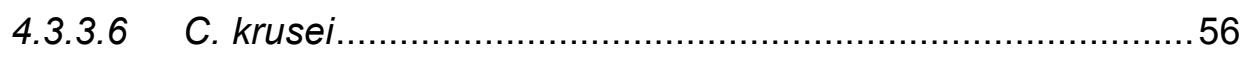

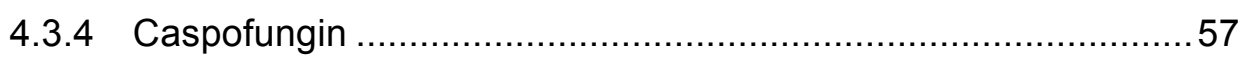

4.3.4.1 C. albicans .............................................................. 57

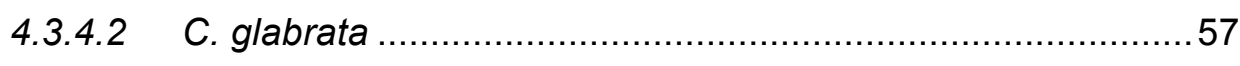

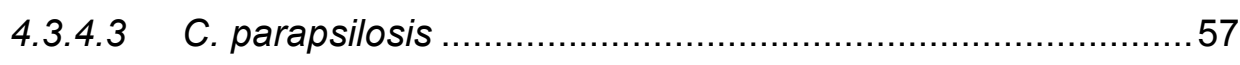

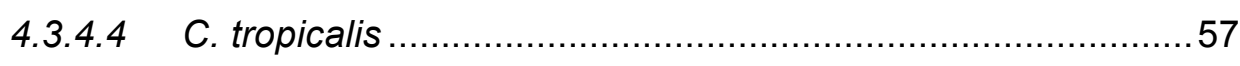

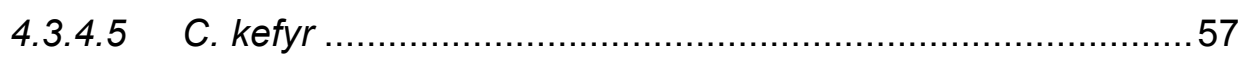

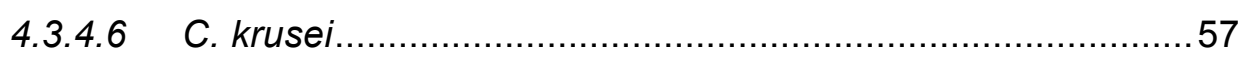

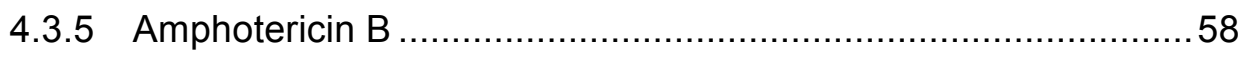

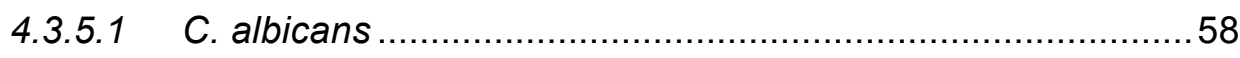

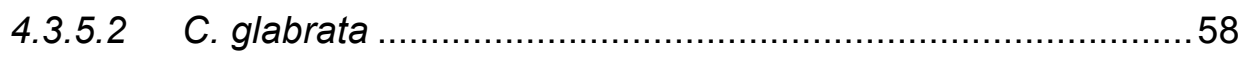




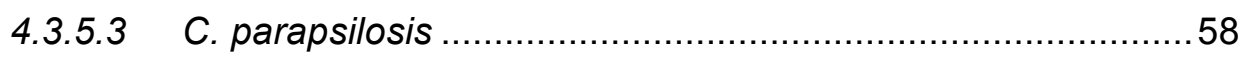

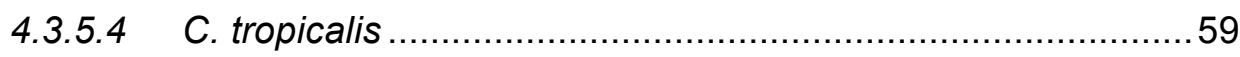

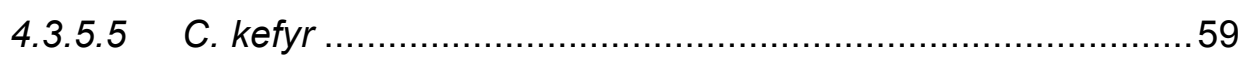

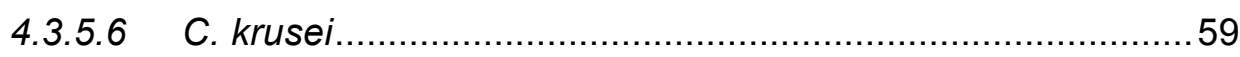

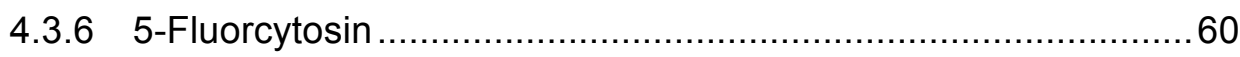

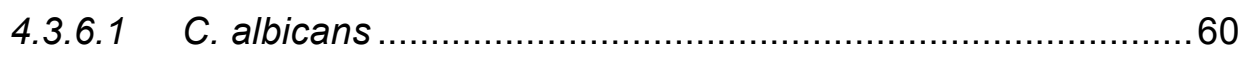

4.3.6.2 C. glabrata ............................................................... 60

4.3.6.3 C. parapsilosis .........................................................60

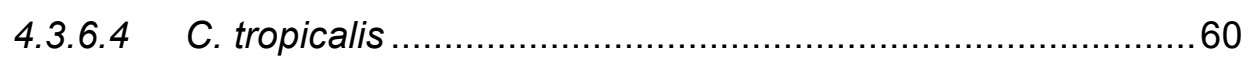

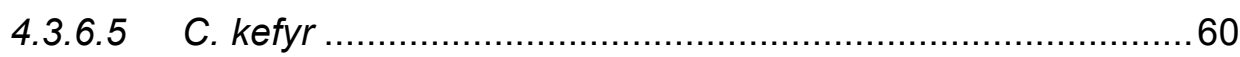

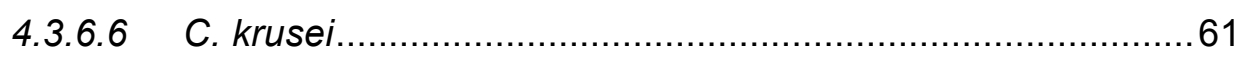

4.3.7 Zusammenfassung aller Etest-Ergebnisse .............................61

4.4 Vergleich von Mikrodilutionstest und Etest .........................................62

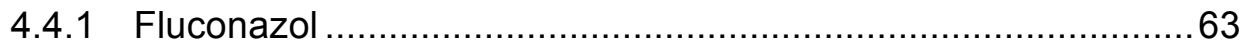

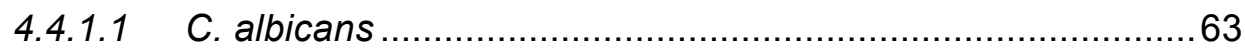

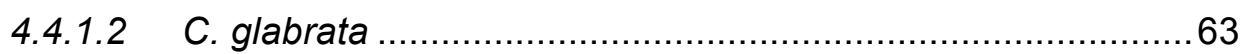

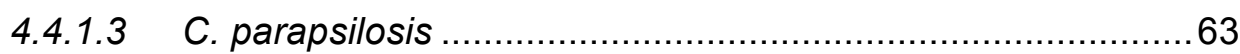

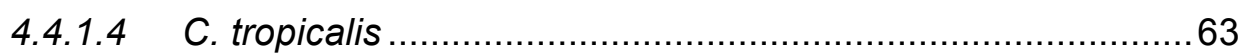

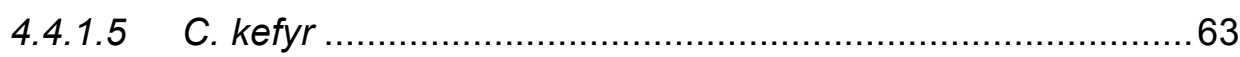

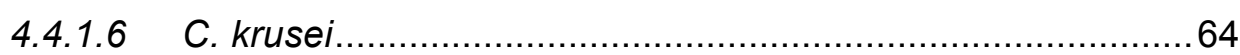

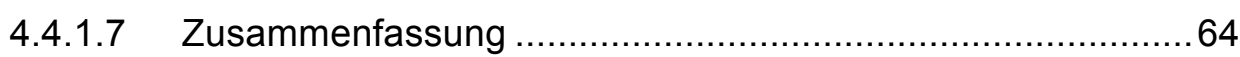

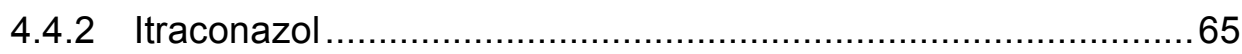

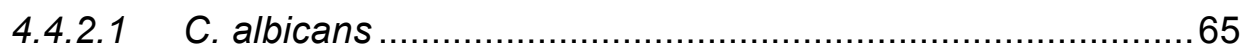

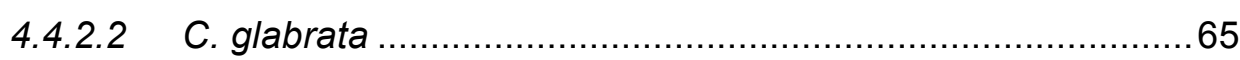

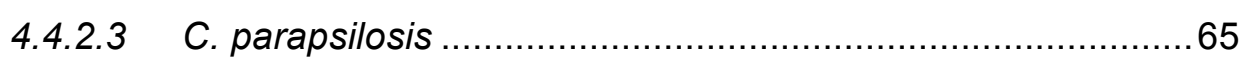

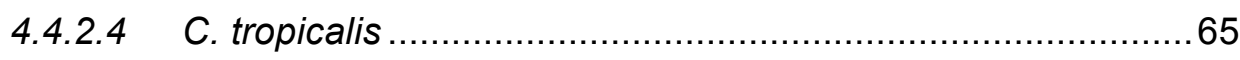

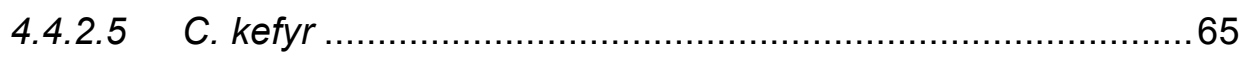

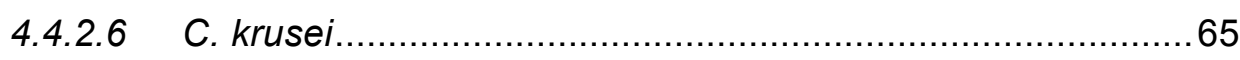

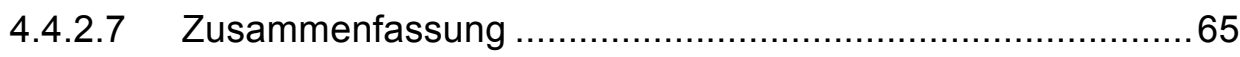

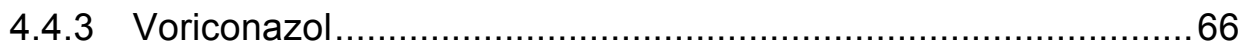

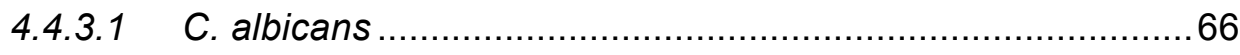




\begin{tabular}{|c|c|}
\hline 1.3.2 & glabrata. \\
\hline 4.3 .3 & C. parapsilosis ...... \\
\hline 4.3 .4 & C. tropicalis ..... \\
\hline 4.3 .5 & 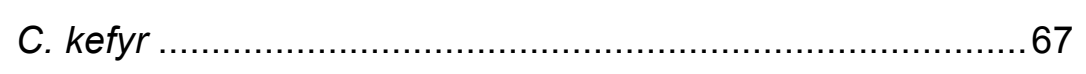 \\
\hline 4.3 .6 & C. krusei... \\
\hline 4.3 .7 & Zusammenfassung .................. \\
\hline $4.4 \mathrm{C}$ & aspofungin .. \\
\hline 4.4 .1 & C. albicans ................ \\
\hline 4.4 .2 & C. glabrata ....... \\
\hline 4.4 .3 & C. parapsilosis . \\
\hline 4.4 .4 & C. tropicalis ...... \\
\hline 4.4 .5 & C. kefyr .............. \\
\hline 4.4 .6 & C. krusei.............. \\
\hline 4.4 .7 & Zusammenfassung ... \\
\hline $4.5 \mathrm{~A}$ & mphotericin B ........... \\
\hline 4.5 .1 & C. albicans ......... \\
\hline 4.5 .2 & C. glabrata .............. \\
\hline 4.5 .3 & C. parapsilosis .... \\
\hline 4.5 .4 & C. tropicalis ............... \\
\hline 4.5 .5 & C. kefyr.. \\
\hline 4.4 .5 .6 & C. krusei.......... \\
\hline 4.5 .7 & Zusammenfassung .. \\
\hline 4.65 & Fluorcytosin ......... \\
\hline 4.6 .1 & C. albicans ........ \\
\hline 4.6 .2 & C. glabrata .......... \\
\hline 4.6 .3 & C. parapsilosis .......... \\
\hline 4.4 .6 .4 & C. tropicalis ........ \\
\hline 4.6 .5 & C. kefyr .............. \\
\hline 4.4 .6 .6 & C. krusei.... \\
\hline 4.6 .7 & 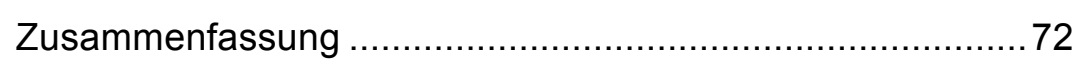 \\
\hline
\end{tabular}




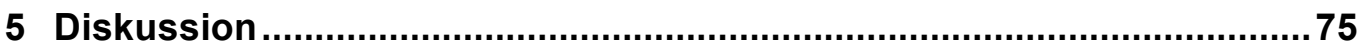

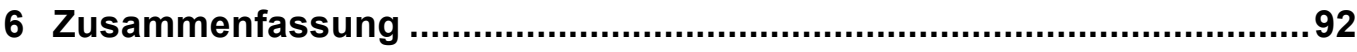

7 Anhang

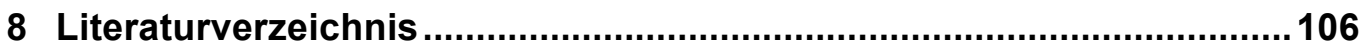




\section{Abkürzungsverzeichnis}

Die verwendeten Abkürzungen entsprechen den im internationalen Einheitensystem vereinbarten Abkürzungen (SI-Einheiten). Übliche Zeichen und Abkürzungen werden hier nicht aufgeführt.

API

ARTEMIS

ATCC

BAL

C.

CLSI

Cr.

d-sensibel

DMSO

ECMM

EIEIO

G.

MHK

MOPS

NCCLS

NRZSM

RPMI

S.

SCOPE

SENTRY
Analytischer Profil-Index

Advanced Research Testbed for Medical Informatics

American Type Culture Collection

Bronchiallavage

Candida

Clinical and Laboratory Standards Institute (früher NCCLS)

Cryptococcus

dosisabhängig sensibel

Dimethylsulfoxid

European Confederation of Medical Mycology

Emerging Infections and the Epidemiology of lowa Organisms

Geotrichum

Minimale Hemmkonzentration

Morpholinproposulfid-Säure

National Committee for Clinical Laboratory Standards

Nationales Referenzzentrum für systemische Mykosen

Zellkulturmedium, das durch das Roswell Park Memorial Institute entwickelt wurde

Saccharomyces

Surveillance and Control of Pathogens of Epidemiologic Importance

Wächter, Überwachung 
Spp.

Spezies (Plural)

Trichosp. Trichosporon

ZVK

Zentraler Venenkatheter 


\section{Abbildungsverzeichnis}

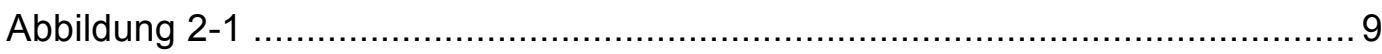

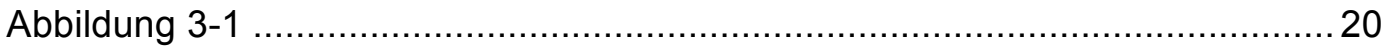

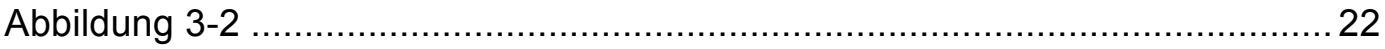

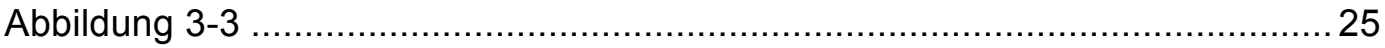

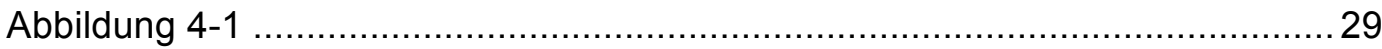

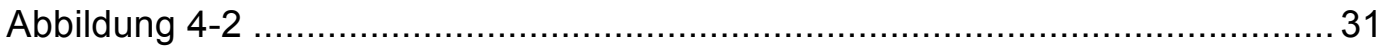

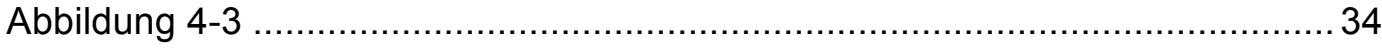

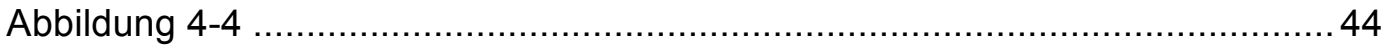




\section{Tabellenverzeichnis}

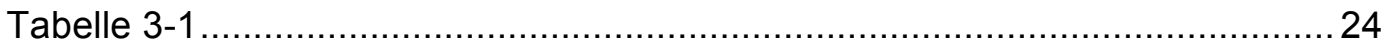

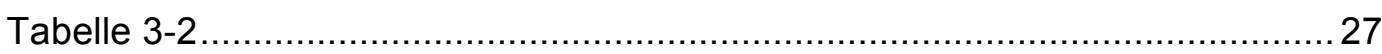

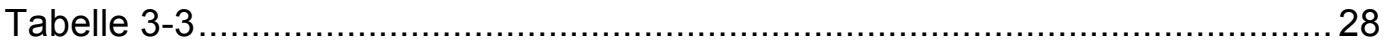

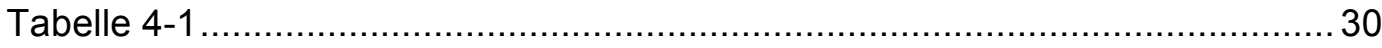

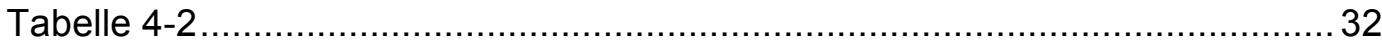

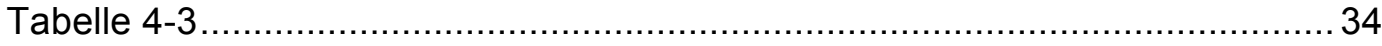

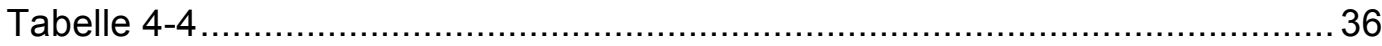

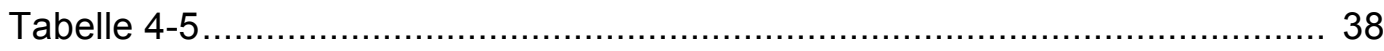

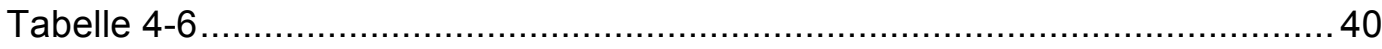

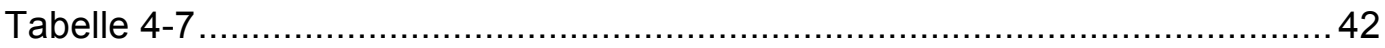

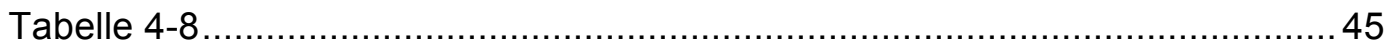

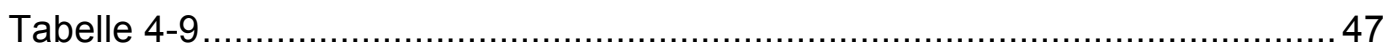

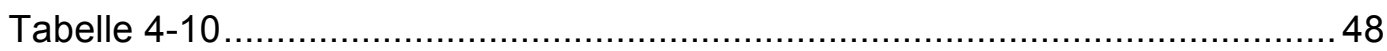

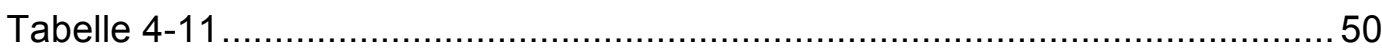

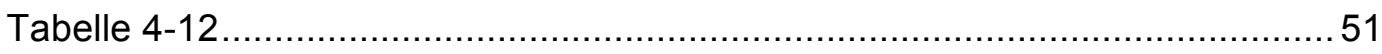

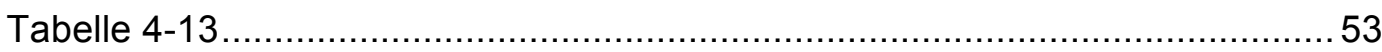

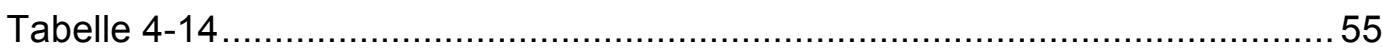

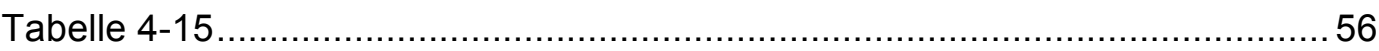

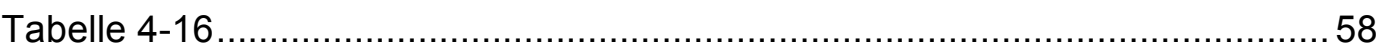

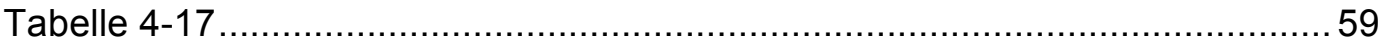

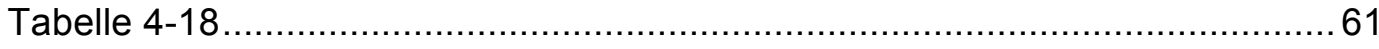

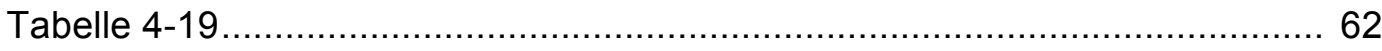

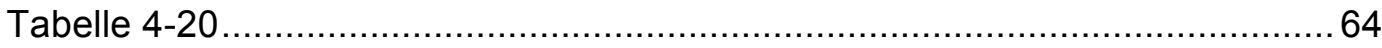




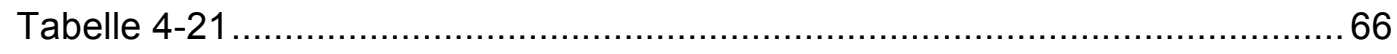

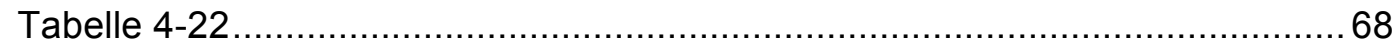

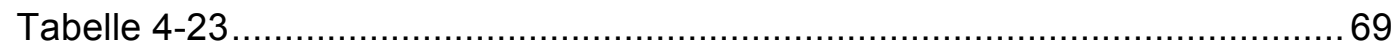

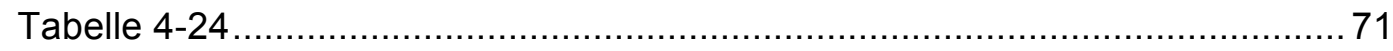

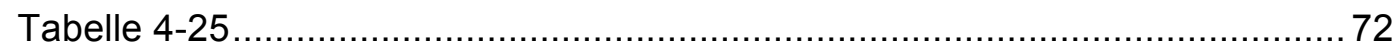

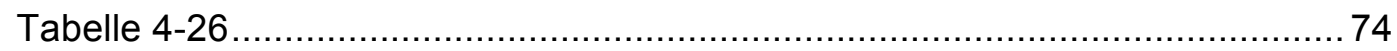

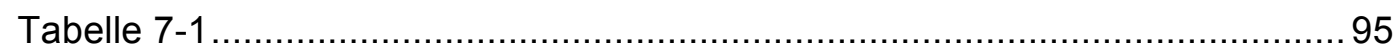

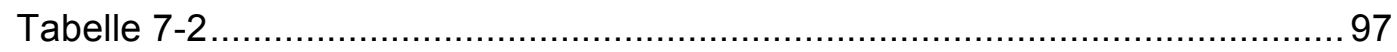

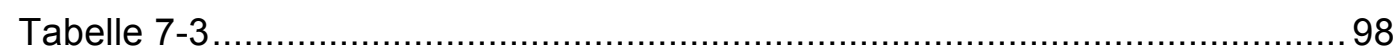

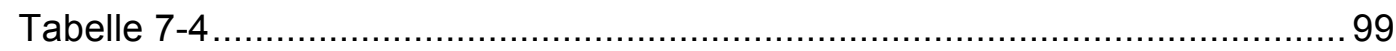

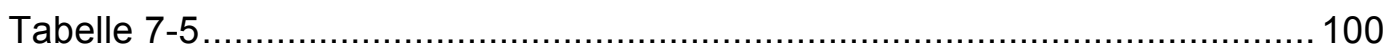

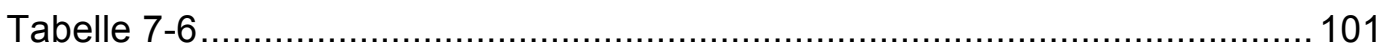

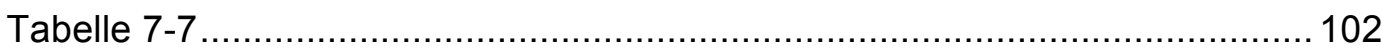

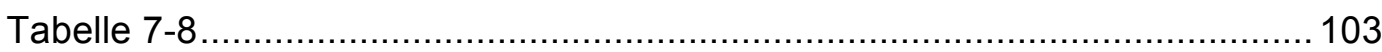




\section{$1 \quad$ Einleitung}

Systemische Mykosen rangieren in Deutschland auf dem fünften, in den USA auf dem vierten Platz der Ursachen nosokomialer Infektionen (Geffers et al. 2004, Edmond et al. 1999, Pfaller et al. 1998a). Während der letzten zwei Dekaden nahm die Inzidenz beständig zu (Pfaller und Diekema 2007). Die invasive Mykose gilt somit als relevantes Problem unseres Gesundheitswesens (Pfaller und Diekema 2002).

Systemische Pilzinfektionen sind durch eine hohe Mortalität gekennzeichnet, die sich doppelt negativ auswirkt, da überwiegend schwer kranke Patienten mit Abwehrschwäche von Candidämien betroffen sind. Daraus resultiert somit eine Mortalität, die höher ist als die aller anderen Erreger nosokomialer Infektionen (Tortorano et al. 2004, Wey et al. 1988, Wenzel und Edmond 2001) und die auf 30-50\% beziffert werden muss (Edmond et al. 1999, Lyytikäinen et al. 2002, Pfaller und Diekema 2007, Pittet et al. 1997). Unter den genannten Voraussetzungen verlaufen die Infektionen oft fulminant. In Deutschland sind Pilzinfektionen für $17,8 \%$ der schweren Verlaufsform einer Sepsis ursächlich (Engel et al. 2007). Diagnostik und Therapie sind dann deutlich erschwert. Rasche und effiziente Therapie setzen Kenntnisse über Speziesverteilung und Resistenzlage voraus, da Differenzierung und Resistenztestung oft erst mehrere Tage nach Gewinnen der Probe vorliegen.

Hinsichtlich der Epidemiologie und Resistenzlage der systemischen Mykosen existieren für Deutschland nur wenige Studien. Sie sind größtenteils regional oder zeitlich eng begrenzt, weisen nur geringe Fallzahlen auf oder beziehen sich auf bestimmte Risikogruppen (Borg-von Zepelin et al. 1993, Büchner et al. 2002, Fleck et al. 2007, Geffers et al. 2004, Glasmacher et al. 1998, Knoke et al. 1997, Rüchel et al. 2002, Schwesinger et al. 2005).

Zur Erhebung repräsentativer Daten generiert das Nationale Referenzzentrum für Systemische Mykosen (NRZSM) eine Stammsammlung aus bundesweiten Pilzisolaten. Für die vorliegende Arbeit wurden die Isolate aus dem Zeitraum Juli 2004 bis August 2005 untersucht.

Haupterreger der nosokomialen Pilzinfektionen sind Candidaspezies; sie stellen 
auch den größten Anteil der für diese Arbeit untersuchten Isolate dar. Neben der Speziesverteilung insgesamt, wurde diese auch in Bezug auf Alter, Geschlecht und Krankenhausabteilung analysiert. Außerdem wurden alle Proben auf ihre Empfindlichkeit gegenüber sechs verschiedener Antimykotika untersucht. Diese Antimykotika finden oder fanden alle einen breiten klinischen Einsatz in Deutschland. Es sind die drei Azole Fluconazol, Itraconazol, Voriconazol, das Echinocandin Caspofungin sowie Amphotericin B und 5-Fluorcytosin. Somit sind sowohl klassische als auch moderne Antimykotika mit erweitertem Wirkspektrum und anderem Nebenwirkungsprofil repräsentiert.

Zur Resistenztestung von Pilzen hat sich die Methode der Mikrodilution als Referenzmethode etabliert. Sie dient der Bestimmung der minimalen Hemmkonzentration und wurde über viele Jahre ständig weiterentwickelt, ist jedoch bis heute zeit- und arbeitsintensiv. Der aktuelle Wissensstand ist in den CLSI-Leitlinien zusammengefasst (CLSI M27-A3 2008, NCCLS M27-A2 2002).

Ein anderes Testverfahren ist der in der Handhabung einfache Etest. Er wird seit langem in anderen Bereichen der mikrobiologischen Resistenztestung verwendet und wurde für die Testung an Pilzen weiterentwickelt. Inzwischen findet er breiten Einsatz im klinischen Alltag.

Die Zielsetzung dieser Arbeit kann folgendermaßen zusammengefasst werden:

1. Sammlung epidemiologischer Daten über systemische Mykosen in Deutschland,

2. Testung der Empfindlichkeit dieser Isolate gegenüber sechs gängigen Antimykotika und

3. Vergleich des Etests mit der Referenzmethode Mikrodilution im Hinblick auf den Routineeinsatz. 


\section{$2 \quad$ Material}

\subsection{Herkunft der Verbrauchsmaterialien und Chemikalien}

Becton, Dickinson \& Company, NJ,USA

Bacto Agar

Tween 80 Polysorbat

Biochrom \& KG, Berlin

Instamed-Medium RPMI 1640

L-Glutamin

Bio- Mérieux, Marcy- I’Etoile, Frankreich

System zur Identifizierung von Hefen API 32 C AUX

Copan Diagnostics, Corona, CA, USA

Sterile Wattetupfer

Fluka Chemie GmbH, Buchs, Schweiz

5-Fluorcytosin

J. T. Baker, Deventer, Niederlande

Methanol

Mast Diagnostica $\mathrm{GmbH}$, Reinfeld

Cryobank Stammhaltungssystem

Merck \& Co, Inc., Whitehouse Station, USA

DMSO

Glucose

Methylenblau

Natriumchlorid

Sabouraud-4\% Glucose-Agar

Sigma-Aldrich Chemie GmbH, Steinheim

Morpholinproposulfid-Säure (MOPS) $\geq 99,5 \%$ Puffer 


\subsection{Herkunft der Antimykotika}

AB Biodisk, Solna, Schweden

Amphotericin-B-Etest

Fluconazol-Etest

5-Fluorcytosin-Etest

Fluka Chemie GmbH, Buchs, Schweiz

5-Fluorcytosin

Sigma-Aldrich Chemie GmbH, Steinheim

Amphotericin B

Die restlichen Antimykotika wurden freundlicherweise durch die folgenden Unternehmen bereitgestellt:

AB Biodisk, Solna, Schweden

Caspofungin-Etest

Itraconazol-Etest

Voriconazol-Etest

MSD Sharp \& Dohme GmbH, Haar

Caspofungin

Ortho Biotech, Neuss

Itraconazol

Pfizer $\mathrm{GmbH}$, Karlsruhe

Fluconazol

Voriconazol 


\subsection{Herkunft der Geräte}

Bio-Mérieux, Marcy-I’ Etoile , Frankreich

Densimat Mc-Farland-Photometer

Brand, Wertheim

Glaspipette $1 \mathrm{ml}, 2 \mathrm{ml}, 5 \mathrm{ml}, 10 \mathrm{ml}, 20 \mathrm{ml}$

Glas-Pasteurpipetten

Transferpette $8 \times 20-200 \mu \mathrm{l}$

Cooke Engineering Company, Alexandria, VA, USA

Ablesespiegel für Mikrotestplatten

Corning Incorporated, Corning, NY, USA

Bottle Top Polyethersulfon-Filter 0,22 $\mu \mathrm{m}$

Dechiney Plastic Packing, Chicago, IL, USA

Parafilm M

Dynex Technologies, Chantilly, VA, USA

MR x TC Revelation Photometer mit 630 nm Filter

Fa Eppendorf, Hamburg

Research Pipette $10 \mu \mathrm{l}, 100 \mu \mathrm{l}, 200 \mu \mathrm{l}, 1000 \mu \mathrm{l}$

Mehrkanal Research Pipette 30-300 $\mu \mathrm{l}$

Multipette plus

Combitips plus $5 \mathrm{ml}$

Flow Laboratories $\mathrm{GmbH}$, Meckenheim

Sterile Werkbank (Laminar Flow) Gelaire HF 48

Greiner GmbH, Frickenhausen

96-Loch Mikrotiterflachbodenplatten

PS-Abdeckplatten

PP-test tubes cellstar $15 \mathrm{ml}, 50 \mathrm{ml}$

Hirschmann Laborgeräte

Pipetus-Akku 
Janke \& Kunkel KG, Staufen im Breisgau

Magnetrührer IKA-Combimag Reo

Kern \& Sohn GmbH, Balingen-Frommern

Waage Typ 572

KNF Neuberger, Freiburg

Vakuumpumpe

Memmert GmbH + CoKG, Schwabach

Brutschrank BE 600

Rettberg, Göttingen

Flachbodengläser Fiolax 80 x $\varnothing 17,75 \times 0,55$ mm

Kulturröhrchen

Sarstedt Aktiengesellschaft \& Co, Nümbrecht

Pipettenspitzen $10 \mu \mathrm{l}, 200 \mu \mathrm{l}, 1000 \mu \mathrm{l}$

Impfschlinge $1 \mu \mathrm{l}, 10 \mu \mathrm{l}$

Scaltec, Heiligenstadt

Feinwaage SBC 33

Schott AG, Mainz

Glasflaschen Duran 50 ml, 100 ml, 200 ml, 500 ml, 1000 ml, 2000 ml

Schütt Labortechnik GmbH, Göttingen

Ph-Meter ph 526

Bunsenbrenner flammy $S$

Scientific Industry, Bohemia, NY, USA

Vortex Genie 2 


\subsection{Herkunft der Referenzstämme}

Als Referenzstämme wurden die beiden Isolate C. krusei ATCC 6258 und C. parapsilosis ATCC 20019 verwendet (Pfaller et al. 1994). Sie stammen ursprünglich aus der American Type Culture Collection (Manassas, Virginia, USA), sind jedoch zur Forschung im Institut für Medizinische Mikrobiologie der Georg-August-Universität Göttingen eingelagert. Mit Hilfe der Referenzstämme konnte eine gleich bleibende Qualität der beiden Testverfahren bei den unterschiedlichen Antimykotika sowie bei der Differenzierung der Hefen sichergestellt werden.

\subsection{Herkunft der Isolate}

Die insgesamt 567 getesteten Isolate gehören zu der Stammsammlung des Nationalen Referenzzentrums für Systemische Mykosen (Institut für Medizinische Mikrobiologie der Georg-August-Universität Göttingen).

Zur Erhebung epidemiologischer Daten über Fungämie bzw. Pilzseptitiden wurden seit dem Jahr 2004 diagnostisch tätige Laboratorien von Universitätskliniken, Krankenhäusern unterschiedlicher Größe sowie Laborpraxen angeschrieben und gebeten, Pilzisolate einzusenden. Teilgenommen haben in diesem Rahmen über dreißig unterschiedliche Labore aus dem ganzen Bundesgebiet. Eine Liste aller Labore, die teilgenommen haben, ist mit Adressen im Anhang aufgeführt (Tabelle 7-8, S. 103). Die Isolate stammen aus primär sterilen Materialien, die über den Zeitraum von Juli 2004 bis August 2005 entnommen wurden.

Zu jedem Isolat gehörte ein Begleitschein, der folgende Informationen enthielt:

- Labor-Nummer des Isolates

- Datum der Materialentnahme

- Herkunft des Isolates

- Initiale der Patientin / des Patienten

- Geburtsdatum und Geschlecht der Patientin / des Patienten

- Einsender 
- klinische Diagnose bzw. Verdachtsdiagnose

- ggf. Daten zur Erkrankung des Patienten/ der Patientin und zur Vortherapie

- voraussichtliche Pilzart des Isolates.

Der Einsendeschein ist in Abbildung 2-1 dargestellt.

Die Isolate wurden auf Pilztransportmedien, wie beispielsweise SabouraudSchrägagar-Röhrchen, verschickt. Im NRZSM wurden sie zunächst erneut mit der API-Methode differenziert. Ein Großteil der Isolate wurde anschließend der Empfindlichkeitstestung mit dem Etest unterzogen. Frisch kultiviert wurden alle Isolate mit Hilfe des Stammhaltungssystems Cryobank bei einer Temperatur von $-27^{\circ} \mathrm{C}$ als Reinkulturen eingelagert. Im Intervall folgte die Testung aller Isolate durch die Methode der Mikrodilution. 


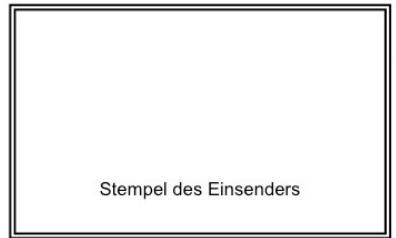

Begleitschein zur Untersuchung eines Pilz-Isolates im

NRZ für Systemische Mykosen

Nationales Referenzzentrum für Systemische Mykosen Institut für Medizinische Mikrobiologie

Universitätsklinikum Göttingen

Kreuzbergring 57

37075 Göttingen

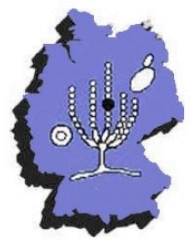

Tel. 0551/395801/ 5806 Fax 0551/ 395861 Email: ugross@gwdg.de mborg@gwdg.de

\section{Angaben zum Pilz-Isolat}

Labor-Nummer des Einsenders:

Datum der Material-Entnahme (TT/MM/JJJJ):

Herkunft des Isolates (Entnahme-Ort bzw. Art des Materials):

Blut $\square$ Liquor $\square$ ZVK-Spitze $\square$ Sonstiges $\square$

Fragestellung:

voraussichtliche Pilzart:

2. Angaben zum Patienten (verschlüsselt)

\begin{tabular}{|l|l|l|}
\hline Vorname, 2. Buchstabe: & Nachname, 3. Buchstabe: & Vom Labor auszufüllen \\
\hline Geburtsjahr & - & Patientencode: \\
\hline Geschlecht & männlich $\square$ weiblich $\square$ & \\
\hline Wohnort (PLZ, erste 3 Ziffern oder Ort) & \\
\hline stationär $\square$ & ambulant $\square$ \\
\hline Krankenhaus: & Behandelnder Arzt: \\
& \\
\hline
\end{tabular}

Klinische Diagnose / Verdacht (mehrere ankreuzen, wenn zutreffend!):

Sepsis

$\square$ Malignom/Hämatol. Erkrankung

Meningitis

HIV-Infektion/AIDS

Pneumonie

Neutropenie

Transplantation, Knochenmark

Transplantation, solides Organ

Intensivmedizinischer Aufenthalt

$\square$ Immunsuppressive Therapie

$\square$ Vorausgegangene Pilzbesiedlung

$\square$ Frühere invasive Mykose

$\square$ Unter antimykotischer Therapie

andere Erkrankung (bitte spezifizieren)

Ort

Datum

\section{Unterschrift}

Name u. Telefonnummer der Kontaktperson

im Labor für Rückfragen

\section{Abbildung 2-1: Begleitschein der Isolate}




\section{$3 \quad$ Methoden}

Die minimale Hemmkonzentration (MHK) eines Antimykotikums ist abhängig von verschiedenen Faktoren. Dazu gehören beispielsweise die Konzentration der Einsaat, die Zusammensetzung und der pH-Wert der Kulturmedien sowie Temperatur und Dauer der Inkubation (Cormican und Pfaller 1996, Rex et al. 1993). Im Folgenden werden die genauen Bedingungen beschrieben, unter denen die Tests der vorliegenden Arbeit durchgeführt wurden.

\subsection{Puffer und Kulturmedien}

\subsubsection{Physiologische Kochsalzlösung}

Die zur Testung der Isolate verwendete Kochsalzlösung wurde durch die Nährbodenküche des Institutes für medizinische Mikrobiologie der Georg-AugustUniversität Göttingen hergestellt. Für die Herstellung eines Liters wurden $9 \mathrm{~g}$ Natriumchlorid in 1 I Aqua dest. gelöst. Daraus ergab sich eine 0,9\%ige Kochsalzlösung. Die Sterilisation erfolgte durch 20minütiges Autoklavieren bei $121^{\circ} \mathrm{C}$. Die Lösung wurde bis zum Verbrauch im keimarmen Kühlraum des Institutes bei einer Temperatur von $8^{\circ} \mathrm{C}$ gelagert.

\subsubsection{Sabouraud-Agar}

Die Anzüchtung der Isolate erfolgte auf Sabouraud-Agar. Dieser wurde ebenfalls von der Nährbodenküche des Institutes angefertigt. Zur Herstellung eines Liters wurden folgende Bestandteile verwendet: $10 \mathrm{~g}$ Pepton, $40 \mathrm{~g}$ Glucose sowie $15 \mathrm{~g}$ Agar-Agar. Diese Zutaten wurden in einem Liter Aqua bidest. gelöst und bei $121^{\circ} \mathrm{C} 15 \mathrm{~min}$ lang autoklaviert. Bei $25^{\circ} \mathrm{C}$ betrug der $\mathrm{pH}$-Wert 5,6 $\pm 0,2$. Es wurde nach Abkühlung der Mischung $1 \mathrm{ml}$ Gentamycin bzw. Chloramphenicol hinzugefügt und anschließend $20 \mathrm{ml}$ des Agar-Gemisches auf eine Petrischale gegeben. Die Höhe der Agarschicht lag damit bei 4,0 $\pm 0,5 \mathrm{~mm}$. Die Lagerung erfolgte in dem genannten Kühlraum.

\subsubsection{RPMI-Medium}

Auch das RPMI-Medium für die Mikrodilution wurde von den Mitarbeitern der Nährbodenküche des Institutes angesetzt. Für die Herstellung eines Liters RPMIMedium waren 10,43g RPMI-Trockenmedium (enthält L-Glutamin und 0,2\% 
(entspricht 2g) Glucose, jedoch kein Bikarbonat) in $900 \mathrm{ml}$ Aqua bidest. zu lösen. Es wurden 34,52g Morpholinproposulfid-Säure (MOPS) hinzugefügt, so dass die Endkonzentration $0,165 \mathrm{~mol} / \mathrm{l}$ betrug. Außerdem beigefügt wurden weitere $2 \mathrm{~g}$ Glucose, so dass die Glucose-Endkonzentration im RPMI-Medium bei 0,4\% lag. Damit wurde bis auf die Verdopplung der Glucose-Konzentration streng nach den NCCLS-Standards verfahren. Anschließend wurde der pH-Wert der Lösung mit $1 \mathrm{Mol} / / \mathrm{NaOH}$ bei $25^{\circ} \mathrm{C}$ auf einen Wert von 7,0 eingestellt. Durch Zusatz von weiterem Aqua bidest. wurde auf ein Volumen von einem Liter aufgefüllt und die Lösung abschließend steril filtriert (Porengröße des Filters $22 \mu \mathrm{m}$ ). Lagerung des Mediums im keimarmen Kühlraum des Institutes.

\subsubsection{RPMI-Agar}

Die für den Etest verwendeten RPMI-Platten wurden durch die Nährbodenküche des Instituts nach folgendem Rezept hergestellt: Es wurden in einem Kolben 10,43g RPMI-Trockenmedium (enthielt L-Glutamin) mit 34,52g MOPS sowie $20 \mathrm{~g}$ Glucose in 1 Aqua dest. gelöst. $0,5 \mathrm{ml}$ Methylenblaulösung wurden hinzugefügt. Der $\mathrm{pH}-$ Wert wurde mit $\mathrm{NaOH}$ auf 7,0 bei $25^{\circ} \mathrm{C}$ eingestellt. Anschließend wurde die Lösung steril filtriert (Porengröße des Filters $22 \mu \mathrm{m}$ ) und auf $45^{\circ} \mathrm{C}$ erwärmt. In einem zweiten Kolben wurden 15g Bacto-Agar in $500 \mathrm{ml}$ Aqua dest. gelöst und danach $20 \mathrm{~min}$ bei $121^{\circ} \mathrm{C}$ autoklaviert. Diese Lösung wurde ebenfalls auf eine Temperatur von $45^{\circ} \mathrm{C}$ gebracht. Beide wurden miteinander vermischt und in Petrischalen zu einer Dicke von 4,0 $\pm 0,5 \mathrm{~mm}$ abgefült. Es fand ebenfalls bis zum Verbrauch die Lagerung im keimarmen Kühlraum des Institutes bei einer Temperatur von $8^{\circ} \mathrm{C}$ statt.

\subsubsection{Reis-Tween-Agar}

Die Anfertigung der Reis-Tween-Agarplatten für die Differenzierung der Hefeisolate erfolgte ebenso durch die Nährbodenküche. Für die Herstellung eines Liters wurden zunächst $25 \mathrm{~g}$ Reis in $750 \mathrm{ml}$ Leitungswasser 45 min lang in einem Dampftopf gekocht. Nach dem Erkalten wurde der Inhalt durch mehrere Lagen Mull filtriert. Anschließend wurde das gewonnene Reiswasser mit $25 \mathrm{~g}$ Agar-Agar versetzt und das Volumen mit Leitungswasser auf $1000 \mathrm{ml}$ aufgefüllt. Die Sterilisation erfolgte durch 20 minütiges Autoklavieren bei $121^{\circ} \mathrm{C}$. Nach Abkühlung des Gemisches auf $50^{\circ} \mathrm{C}$ wurde dem Agar $8 \mathrm{ml}$ Tween 80 (Polysorbat) 
beigemengt. Abschließend wurde der Agar zu einer dünnen Schicht in Petrischalen gegossen. Bis zum Verbrauch erfolgte die Lagerung im keimarmen Kühlraum des Institutes bei einer Temperatur von $8^{\circ} \mathrm{C}$.

\subsection{Differenzierung der Hefen}

Zur Identifizierung der eingeschickten Isolate wurde das System API-20 C AUX bzw. API-32 C AUX verwendet. Dabei wurde zunächst einmal der API-20 C AUX eingesetzt (19 Assimilationsreaktionen und eine Wachstumskontrolle ermöglichten die Identifizierung 44 unterschiedlicher Spezies). Ergaben sich unklare Ergebnisse, so wurde der API-32 C AUX-Test durchgeführt. Dieser ermöglichte die Testung der Reaktionen mit weiteren Substraten (31 Assimilationsreaktionen und eine Wachstumskontrolle, Identifizierung 62 unterschiedlicher Spezies möglich). Die Testung erfolgte an Übernachtreinkulturen, die auf Sabouraud-Agar kultiviert wurden.

Zur Durchführung des Tests wurde die Inkubationswanne zunächst durch Zugabe von etwa $5 \mathrm{ml}$ Aqua dest. zu einer feuchten Kammer umgewandelt. Der Teststreifen wurde dann in die Inkubationswanne eingebracht. In $2 \mathrm{ml}$ des API$\mathrm{NaCl}-0,85 \%-M e d i u m s$ wurde, durch Zugabe einzelner Kolonien einer Übernachtreinkultur des zu differenzierenden Isolates, eine Trübung entsprechend einem Standard-McFarland von 2 eingestellt. 100 $\mu$ l (API-20 C

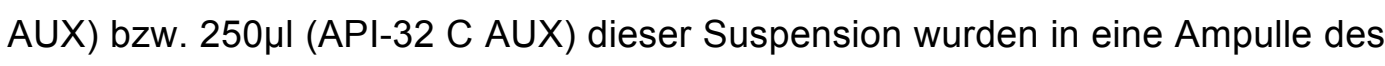
API-C-Mediums eingebracht und mit Hilfe einer Pipette vorsichtig vermischt. Die dadurch entstandene Lösung wurde, unter Vermeidung von Blasenbildung, auf die einzelnen Reaktionsbecher des API-Teststreifens verteilt. Dabei wurden diese so gefüllt, dass die Suspensionsoberfläche leicht konvex verlief. Nach Verschluss der Inkubationswanne wurde für $48-72 \mathrm{~h}$ bei $29^{\circ} \mathrm{C}$ inkubiert.

Die Hefen wuchsen nur dann, wenn sie das vorliegende Substrat verwerten konnten. Das Ablesen der Ergebnisse erfolgte durch Vergleich der Trübungen in den Reaktionsbechern mit der Wachstumskontrolle, die kein Substrat enthielt. Die Becher, die dabei nach Ablauf einer biochemischen Reaktion mit dem Substrat eine stärkere Trübung als die Wachstumskontrolle aufwiesen, konnten als positive Reaktion detektiert werden. Das Resultat wurde auf dem 
Ergebnisblatt ebenso eingetragen, wie das Vorhandensein von Hyphae (Mycel) oder Pseudohyphae (Pseudomycel). Der Nachweis des Bestands von Mycelien erfolgte nach Kultur des Hefeisolates auf Reis-Tween-Agar mikroskopisch.

Die Zuordnung des Ergebnisses zu einer bestimmten Spezies erforderte die Erstellung eines numerischen Profils des Isolates. Die unterschiedlichen Substrate waren durch den Hersteller auf dem Teststreifen jeweils zu Dreiergruppen zusammengefasst worden. Positiven Reaktionen des ersten Tests der Dreiergruppe wurde als Wert eine „Eins“ zugeordnet, dem zweiten Test eine „Zwei“ und dem dritten Test eine „Vier“. Negative Reaktionen erhielten den Wert „Null“. Durch Addition der Zahlenwerte jeder Position konnte das vollständige siebenstellige numerische Profil erstellt werden. Anhand der Profilliste des Herstellers konnte das jeweilige Ergebnis einer bestimmten Spezies zugeordnet werden.

Die Qualitätskontrolle erfolgte mit den Stämmen der American Type Culture Collection (vgl. Abschnitt 2.4).

\subsection{Lagerung der Hefeisolate}

Die Hefeisolate wurden mit Hilfe des Stammhaltungssystems Cryobank eingelagert. Das System besteht aus kleinen Kunststoffröhrchen, die jeweils 25 chemisch behandelte Glaskügelchen sowie $1 \mathrm{ml}$ einer Konservierungslösung enthalten.

Die Einlagerung erfolgte ausschließlich von Material frisch kultivierter Reinkulturen. Nach genauer Beschriftung eines Kunststoffröhrchens wurden einzelne Kolonien mit Hilfe einer Impföse eingebracht. Dies erzielte eine deutliche Trübung der Konservierungslösung, entsprechend einer StandardMcFarland-Trübungsmessung von 3-4. Nach Verschluss des Röhrchens wurde dies vorsichtig geschüttelt, um eine möglichst optimale Verteilung des Organismus in dem Röhrchen zu erzielen. Anschließend erfolgte die Entfernung der Konservierungslösung durch möglichst vollständiges Abziehen mit einer Pipette. Abschließend konnte das Röhrchen gut verschlossen in den dafür vorgesehenen Kästchen bei $-27^{\circ} \mathrm{C}$ eingelagert werden.

Bei der Rekultivierung der Organismen war darauf zu achten, dass die Röhrchen 
nicht auftauten. Daher wurden sie für die Dauer des Vorgangs auf einem Eisbett gelagert. Einzelne Kügelchen konnten mit Hilfe steriler Impfösen entnommen und auf Sabouraud-Agar ausgestrichen werden. Die Inkubation erfolgte für die Dauer von 24 bis $48 \mathrm{~h}$ bei $35^{\circ} \mathrm{C}$ im Brutraum des Instituts.

\subsection{Mikrodilutionstest}

\subsubsection{Präparation des Inokulums}

Die Präparation des Inokulums erfolgte entsprechend der NCCLS-Leitlinien M27A2 (2002). Es wurden jeweils frisch angezüchtete Reinkulturen verwendet, die bei $35^{\circ} \mathrm{C}$ inkubiert wurden und nicht älter als 24 bis $48 \mathrm{~h}$ waren. Einzelne Kolonien der Kulturen wurden in steriler Kochsalzlösung gelöst, bis die Trübung, abhängig von der Spezies, einem Standard-McFarland von 0,3 (C. glabrata) bis 0,5-1 (alle anderen Candida spp.) entsprach. Die Einsaat der Ausgangssuspension lag durch diese Methode bei $1 \times 10^{6}$ bis $5 \times 10^{6}$ Zellen $/ \mathrm{ml}$. Die Zellzahl der Suspension wurde regelmäßig überprüft (vgl. Absatz 3.4.2).

Die Ausgangssuspension wurde durch Zusatz von steriler Kochsalzlösung im Verhältnis von 1:100 und anschließend durch RPMI-Medium im Verhältnis von 1:20 weiter verdünnt. Es lag somit eine Zellkonzentration von $5 \times 10^{2}$ bis $2,5 \times 10^{3}$ Zellen/ml vor. Das Inokulum wurde unmittelbar nach der Herstellung zur Beschickung der Mikrotestplatten verwendet.

\subsubsection{Kontrolle der Einsaat}

Die Etablierung der korrekten Einstellung der Standard-McFarlandTrübungsmessung und die im Verlauf der Testungen durchgeführten stichprobenartigen Kontrollen der Quantität der Einsaat konnten folgendermaßen gewährleistet werden: Zunächst wurden jeweils zweimal $50 \mu \mathrm{l}$ des Inokulums eines Isolates entnommen und unter Zuhilfenahme eines sterilen Glasspatels auf zwei unterschiedliche Sabouraud-Platten gleichmäßig verteilt. Anschließend erfolgte eine $24-$ bis 48 -stündige Bebrütung bei $35^{\circ} \mathrm{C}$. Danach wurden die makroskopisch sichtbaren Kolonien ausgezählt und ein Mittelwert der erzielten Werte beider Platten gebildet. Durch Hochrechnung der Kolonien, die aus den 50 $\mu \mathrm{l}$ des Inokulums stammten, auf $1 \mathrm{ml}$ wurde sichergestellt, dass die Einsaat durchgehend bei $1 \times 10^{6}$ bis $5 \times 10^{6}$ Zellen $/ \mathrm{ml}$ lag . 


\subsubsection{Präparation der Antimykotika-Stammlösungen}

Verwendet wurden die sechs verschiedenen Antimykotika Fluconazol, Itraconazol und Voriconazol sowie Caspofungin, Amphotericin B und 5-Fluorcytosin. Für jedes der einzelnen Antimykotika wurde zunächst eine Stammlösung angesetzt. Die Lagerung dieser Stammlösungen erfolgte bei $-27^{\circ} \mathrm{C}$.

\subsubsection{Fluconazol}

Für die Fluconazol Stammlösung wurden 25,6mg der Fluconazol-Reinsubstanz in $1 \mathrm{ml}$ Methanol angesetzt. Die Konzentration lag damit bei $25,6 \mathrm{mg} / \mathrm{ml}$. Die Entscheidung, Fluconazol entgegen der NCCLS-Leitlinien nicht in Aqua bidest., sondern in Methanol zu lösen, beruht auf dem schlechten Lösungsverhalten in destilliertem Wasser. Das deutlich bessere Lösungsverhalten in Methanol beschrieben bereits Schmalreck und Fegeler (1996).

\subsubsection{Itraconazol, Voriconazol und Amphotericin B}

4,8 mg Reinsubstanz aller drei Antimykotika wurden jeweils in 3ml DMSO gelöst. Die Konzentration der Stammlösung entsprach damit $1600 \mu \mathrm{g} / \mathrm{ml}$. Zur Vermeidung von Konzentrationsschwankungen durch Adhäsion der lipophilen Antimykotika an Plastikoberflächen wurden bei allen Herstellungsschritten ausschließlich Glasgefäße verwendet.

\subsubsection{Caspofungin}

Für den Ansatz der Caspofungin-Stammlösung wurde Aqua bidest. verwendet. Dazu wurde 4,8mg Reinsubstanz verwendet; die Konzentration der Stammlösung lag somit bei $1600 \mu \mathrm{g} / \mathrm{ml}$. Wegen der Lichtempfindlichkeit des Stoffes Caspofungin wurden sowohl die Grundsubstanz als auch Caspofungin enthaltende Lösungen konsequent vor Licht geschützt.

\subsubsection{5-Fluorcytosin}

Für die Stammlösung des Antimykotikums 5-Fluorcytosin wurden 12,8mg Reinsubstanz in $1 \mathrm{ml}$ Aqua bidest. gelöst. Die Konzentration der Stammlösung lag damit bei $12,8 \mu \mathrm{g} / \mathrm{ml}$. 


\subsubsection{Präparation der Verdünnungsreihen}

Die Antimykotika-Suspensionen für die Verdünnungsreihe wurden nach den NCCLS-Richtlinien hergestellt (NCCLS M27-A2 2002).

Im Folgenden wird die Erstellung einer Verdünnungsreihe exemplarisch für die Bestückung einer einzelnen Mikrotestplatte beschrieben: Dazu musste von jeder gewünschten Verdünnungsstufe $1 \mathrm{ml}$ der Lösung erstellt werden. Eine Verdünnungsreihe bestand aus jeweils zehn verschiedenen Verdünnungsstufen. Die Konzentration in jeder einzelnen Position der Mikrotestplatte war dabei so zu wählen, dass sie doppelt so hoch war wie die im eigentlichen Test erwünschte Zielkonzentration, da sich durch spätere Zugabe des Inokulums die Konzentration wiederum halbierte.

Aus einer Stammlösung wurden nur zwei Verdünnungsstufen direkt erstellt. Für die weiteren Verdünnungsstufen mussten Zwischenverdünnungen mit geeignetem Verdünnungsmedium erzeugt werden, da sonst die zu pipettierenden Mengen zu klein ausgefallen wären und exaktes Arbeiten nicht garantiert gewesen wäre. Als Verdünnungsmedium diente für die in DMSO gelösten Antimykotika DMSO und für die in Methanol oder Aqua bidest. gelösten Antimykotika Aqua bidest.

Die einzelnen Verdünnungsstufen der Antimykotika wurden jeweils getrennt angesetzt und gelagert. Die Mikrotestplatten wurden zur Erleichterung des Arbeitsablaufs bereits vor der Testung mit den Verdünnungsstufen beschickt und dann bei mindestens $-27^{\circ} \mathrm{C}$ gelagert. Am Testtag konnten die Platten auf Zimmertemperatur äquilibriert und anschließend mit den frisch angefertigten Hefesuspensionen versehen werden.

\subsubsection{Fluconazol}

Für die Beschickung der Mikrotestplatten mit Fluconazol wurde dabei wie folgt vorgegangen: Fluconazol lag als Stammlösung $(25,6 \mathrm{mg} / \mathrm{ml}=25600 \mu \mathrm{g} / \mathrm{ml})$ in Methanol gelöst vor (vgl. Abschnitt 3.4.3.1).

Für die Erstellung der ersten Konzentrationsstufe $(256 \mu \mathrm{g} / \mathrm{ml})$ wurde die Stammlösung 1:100 mit Mikrodilutionsmedium verdünnt (10 $\mu$ Stammlösung in 990ml RPMI-Medium). 
Für die zweite Konzentrationsstufe $(128 \mu \mathrm{g} / \mathrm{ml})$ wurde die Stammlösung 1:200 mit Mikrodilutionsmedium verdünnt (5 $\mu$ l in 995ml RPMI-Medium).

Zur Erstellung der dritten Konzentrationsstufe $(64 \mu \mathrm{g} / \mathrm{ml})$ musste die Stammlösung zunächst 1:2 mit Aqua bidest. verdünnt werden. Diese Lösung wurde ebenfalls 1:200 mit Mikrodilutionsmedium versetzt $(5 \mu l$ in $995 \mathrm{ml}$ RPMIMedium).

Für die Fertigung der vierten Konzentrationsstufe $(32 \mu \mathrm{g} / \mathrm{ml})$ wurde die Stammlösung in einem separaten Ansatz 1:4 mit Aqua bidest. verdünnt und diese Lösung wiederum 1:200 mit Mikrodilutionsmedium verdünnt.

Die fünfte Konzentrationsstufe $(16 \mu \mathrm{g} / \mathrm{ml})$ wurde angesetzt, indem die Stammlösung 1:8 mit Aqua bidest. verdünnt und diese Lösung ebenfalls 1:200 mit Mikrodilutionsmedium ( $5 \mu$ in 995ml RPMI-Medium) weiterverdünnt wurde.

Das Verdünnungsschema 1:2, 1:4 und 1:8 mit anschließender Zugabe von Mikrodilutionsmedium blieb für die nachfolgenden Konzentrationsstufen bestehen.

$\mathrm{Ab}$ der sechsten Konzentrationsstufe $(8 \mu \mathrm{g} / \mathrm{ml})$ wurde die FluconazolStammlösung nicht mehr direkt eingebracht. Statt dessen wurde die Zwischenverdünnung, die bei der Erstellung der fünften Konzentrationsstufe entstanden war (Stammlösung 1:8 mit Aqua bidest. verdünnt), verwendet. Diese wurde im Verhältnis 1:2 mit Aqua bidest. versetzt und die neu entstandene Suspension wiederum 1:200 mit Mikrodilutionsmedium $(5 \mu \mathrm{l}$ in $995 \mathrm{ml}$ RPMIMedium) verdünnt.

Für die siebte Konzentrationsstufe $(4 \mu \mathrm{g} / \mathrm{ml})$ wurde die Zwischenverdünnung, die bei der Erstellung der fünften Konzentrationsstufe entstanden war (Stammlösung 1:8 mit Aqua bidest. verdünnt), 1:4 mit Aqua bidest. versetzt und danach 1:200 mit Mikrodilutionsmedium (5 $\mu \mathrm{l}$ in 995ml RPMI-Medium) versetzt.

Für die Fertigung der achten Konzentrationsstufe $(2 \mu \mathrm{g} / \mathrm{ml})$ wurde die Zwischenverdünnung, die bei der Erstellung der fünften Konzentrationsstufe entstanden war (Stammlösung 1:8 mit Aqua bidest. verdünnt), 1:8 mit Aqua bidest. vermischt und diese Lösung 1:200 mit Mikrodilutionsmedium weiterverdünnt. 
Zur Erstellung der neunten Konzentrationsstufe $(1 \mu \mathrm{g} / \mathrm{ml})$ wurde die bei der Erstellung der achten Konzentrationsstufe entstandene Zwischenverdünnung (Stammlösung 1:64 mit Aqua bidest. verdünnt) im Verhältnis 1:2 mit Aqua bidest.

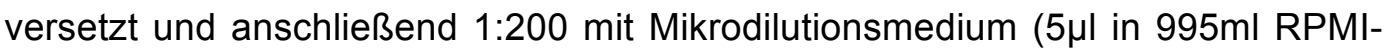
Medium) verdünnt.

Für die zehnte Konzentrationsstufe $(0,5 \mu \mathrm{g} / \mathrm{ml})$ wurde die bei der Erstellung der achten Konzentrationsstufe entstandene Zwischenverdünnung (Stammlösung 1:64 mit Aqua bidest. verdünnt), 1:4 mit Aqua bidest. versetzt und ebenfalls 1:200 im Mikrodilutionsmedium ( $5 \mu$ in 995ml RPMI-Medium) eingesetzt.

Die Endkonzentrationen nach Zugabe der Hefesuspension lagen bei $128 \mu \mathrm{g} / \mathrm{ml}$ bis $0,25 \mu \mathrm{g} / \mathrm{ml}$.

\subsubsection{Itraconazol, Voriconazol und Amphotericin B}

Die Verdünnungsreihe für diese drei Antimykotika wurde entsprechend dem Vorgehen bei Fluconazol hergestellt.

Abweichend davon wurde die jeweils doppelte Menge von AntimykotikaStammlösung verwendet, so dass sich die Antimykotika-Konzentration halbierte.

Damit lag die Verdünnung der Antimykotika-Stammlösung mit Mikrodilutionsmedium bei 1:50 für die erste Konzentrationsstufe und 1:100 für die zweite Konzentrationsstufe (anstelle von 1:100 und 1:200 für die jeweiligen Konzentrationsstufen bei Fluconazol).

Des Weiteren wurde bei schlechter Wasserlöslichkeit dieser drei Antimykotika zur Verdünnung DMSO anstelle des Aqua bidest. verwendet.

Die Endkonzentrationen nach Zugabe der Hefesuspension lagen hier bei $16 \mu \mathrm{g} / \mathrm{ml}$ bis $0,0313 \mu \mathrm{g} / \mathrm{ml}$.

\subsubsection{Caspofungin}

Die Verdünnungsreihe mit Caspofungin wurde analog dem Vorgehen bei Amphotericin B, Itraconazol und Voriconazol hergestellt. Anstelle von DMSO wurde jedoch in diesem Fall für die Verdünnungen Aqua bidest. verwendet. Wegen der Lichtempfindlichkeit des Stoffes Caspofungin wurden sowohl die Reinsubstanz als auch alle Caspofungin-enthaltenden Lösungen konsequent vor Licht geschützt. Hier lagen die Endkonzentrationen nach Zugabe der 
Hefesuspension ebenfalls bei $16 \mu \mathrm{g} / \mathrm{ml}$ bis $0,0313 \mu \mathrm{g} / \mathrm{ml}$.

\subsubsection{5-Fluorcytosin}

Die Verdünnungsreihe mit der 5-Fluorcytosin-Stammlösung entsprach dem bei Fluconazol beschriebenen Vorgehen, begann jedoch erst mit einer Konzentration der Stammlösung von $128 \mu \mathrm{g} / \mathrm{ml}$, so dass die Zielkonzentrationen nach Zugabe der Hefesuspension hier bei $64 \mu \mathrm{g} / \mathrm{ml}$ bis $0,125 \mu \mathrm{g} / \mathrm{ml}$ lagen.

\subsubsection{Beschickung der Mikrotestplatten}

Die Beschickung der Mikrotestplatten erfolgte unter sterilen Bedingungen. Einem Isolat waren dabei jeweils zwei übereinander liegende Zeilen zugeordnet, so dass ein Isolat durch zwei Testansätze immer doppelt getestet wurde. Die erste der zwölf Reihen einer Mikrotestplatte enthielt die Wachstumskontrolle, die zweite bis elfte Reihe die zehn Verdünnungsstufen der Antimykotika in absteigender Konzentration und die zwölfte Reihe enthielt die Sterilkontrolle. Die Abbildung 3-1 verdeutlicht die Anordnung der Reihen. In einem ersten Schritt erfolgte die Beschickung mit den Antimykotika-Suspensionen und dem RPMIMedium und erst zum Schluss wurde das Inokulum hinzugegeben.

\subsubsection{Antimykotika-Suspensionen und RPMI-Medium}

Zunächst wurden die Mikrotestplatten mit den unterschiedlichen Verdünnungsstufen der Antimykotika sowie mit dem Mikrodilutionsmedium beschickt.

Die Wachstumskontrolle enthielt $100 \mu \mathrm{l}$ des RPMI-Mediums, jedoch kein Antimykotikum. Die zweite Reihe erhielt die Antimykotikum-Suspension der jeweils niedrigsten Konzentrationsstufe. Die Suspensionen der unterschiedlichen Verdünnungsstufen wurden mit zunehmender Konzentration in die zweite bis elfte Reihe eingebracht. Die Sterilkontrolle enthielt $200 \mu l$ des RPMI-Mediums.

Die beschickten Mikrotestplatten wurden steril verpackt und bei einer Temperatur von $-27^{\circ} \mathrm{C}$ gelagert. Am Testtag wurden sie rechtzeitig auf Raumtemperatur gebracht, so dass sie zu Beginn der Beschickung mit dem Inokulum vollständig aufgetaut waren. 


\subsubsection{Inokulum}

Als abschließender Arbeitsschritt wurden die Pilzisolate auf die wie eben beschrieben vorbestückten Mikrotestplatten aufgebracht. Dazu wurden, nach gründlicher Durchmischung, $100 \mu l$ des frisch angesetzten Inokulums in jede der Positionen, außer in die Reihe der Sterilkontrolle, gegeben. Zuletzt enthielt jede

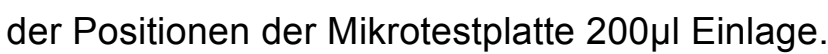

Die unterschiedlichen Konzentrationsstufen der einzelnen Antimykotika einer fertig beschickten Mikrotestplatte wird durch das nachfolgend stehende Schema der Abbildung 3-1 verdeutlicht.

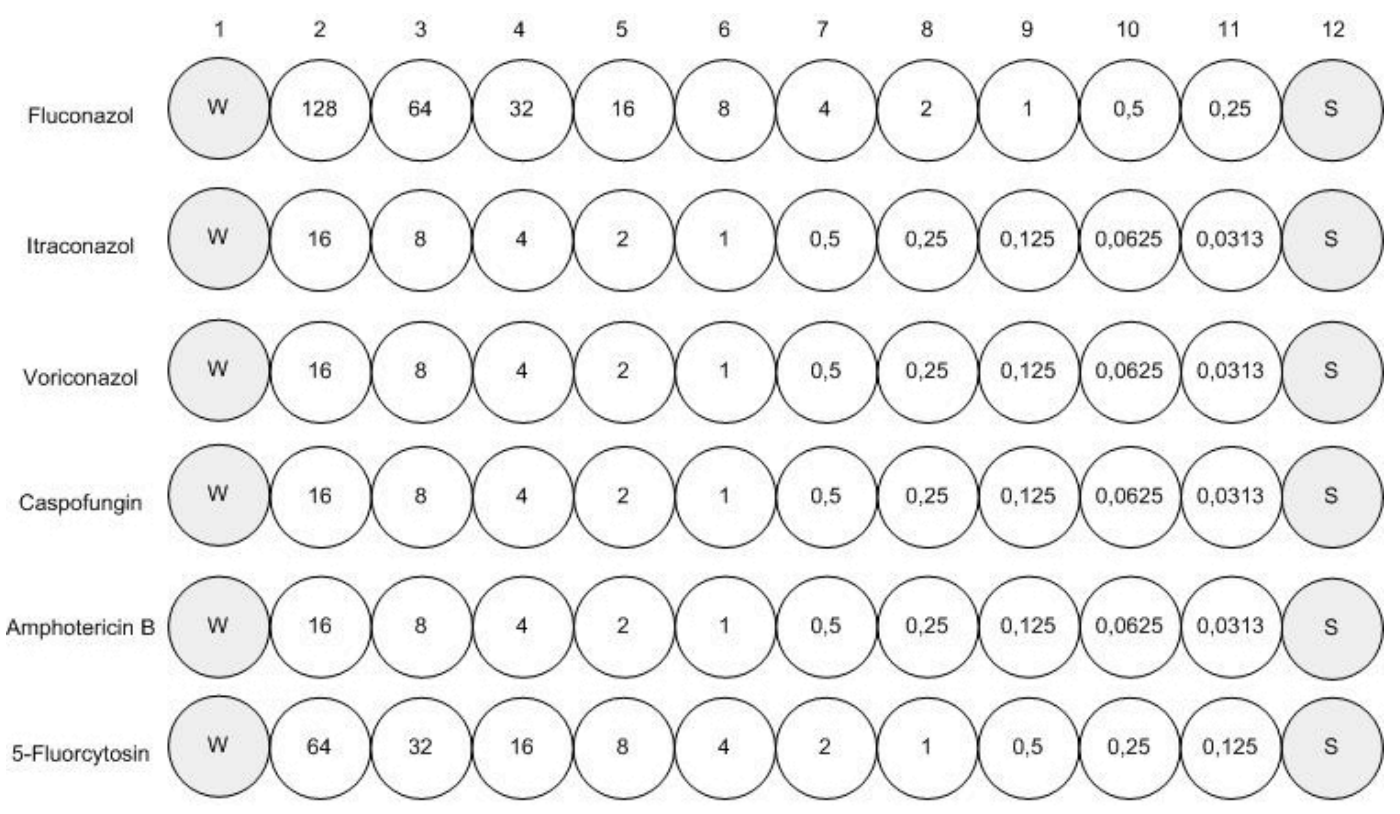

Abbildung 3-1: Endgültige Verdünnungsstufen der Antimykotika in $\mu \mathrm{g} / \mathrm{ml}$ nach Zugabe des Inokulums veranschaulicht auf einer Mikrotestplatte. Reihe 1 entspricht der Wachstumskontrolle, Reihe 12 der Sterilkontrolle.

\subsubsection{Inkubation}

Die fertig beschickten Mikrotestplatten wurden in einer keimarmen feuchten Kammer im temperaturkontrollierten Brutschrank bei $35^{\circ} \mathrm{C}$ für die Dauer von $24 \mathrm{~h}$ bzw. 48h für C. parapsilosis inkubiert (Rex et al. 2001, Lozano-Chiu et al. 1999, Revankar 1998). Die Isolate der Spezies Cr. neoformans und S. cerevisiae bedurften jedoch einer Inkubation von bis zu $72 \mathrm{~h}$, da in der Regel vorher zur Interpretation des Ergebnisses keine ausreichende optische Dichte vorlag. 


\subsubsection{Ablesen der Ergebnisse}

Die Ablesung der Mikrotestplatten erfolgte durch zwei unterschiedliche Verfahren.

\subsubsection{Makroskopisches Ablesen}

Zunächst erfolgte eine makroskopische Kontrolle des Wachstums der Hefen. Hierzu wurde der oben aufgeführte Ablesespiegel für Mikrotestplatten verwendet (vgl. Abschnitt 2.2). Jede Position wurde beurteilt und einer der Kategorien „kein Wachstum“, „geringes Wachstum", „normales bis kräftiges Wachstum“ oder „Kontamination“ zugeordnet. Diese Ergebnisse dienten dem Abgleich mit den photometrisch erhobenen Ergebnissen, waren aber nicht die Grundlage zur Ermittlung der endgültig festgehaltenen minimalen Hemmkonzentrationen, da eine genaue Bestimmung der MHK durch das Phänomen des „trailing growth“ bei fungistatischen Antimykotika schwierig ist (Davey et al. 1998). Dabei kommt es v.a. bei $C$. albicans und $C$. tropicalis trotz hoher Konzentrationen fungistatischer Antimykotika, zu geringem, aber persistierendem Wachstum des Isolates. Das Wachstum erfolgt noch vor dem Einsetzen der fungistatischen Wirkung des Antimykotikums, so dass die Wirksamkeit verfälscht wird. Eigentlich sensible Stämme können dadurch resistent erscheinen (Arikan 2007, Arthington-Skaggs et al. 2002).

\subsubsection{Photometrisches Ablesen}

In einem zweiten Schritt erfolgte die Ablesung spektrophotometrisch unter Verwendung des MR x TC Revelation Photometers mit einem Filter der Wellenlänge von 630nm. Dazu musste vor der Messung jede einzelne Position der Mikrotestplatte durchmischt werden, damit anschließend photometrisch die optische Dichte bestimmt werden konnte (Anaissie et al. 1996, Ghannoum et al. 1992). Anhand der gemessenen Werte konnte nach Bildung des Mittelwertes der beiden zu einem Isolat gehörenden Ergebnisse jeweils die minimale Hemmkonzentration ermittelt werden. Sie war definiert als diejenige Konzentration des Antimykotikums, bei der es zu einer vollständigen Inhibition des Hefewachstums im Vergleich zur Wachstumskontrolle kam. Die MHK ${ }_{50}$ bzw. $\mathrm{MHK}_{90}$ beschrieben dementsprechend eine Reduktion des Wachstums um 50\% bzw. 90\% im Vergleich zur Wachstumskontrolle. 
Für Amphotericin B wurde die MHK bei $90 \%$ oder darüber, d.h. bei der Antimykotika-Konzentration, bei der nur minimales bzw. kein Wachstum erfolgte, abgelesen.

Für 5-Fluorcytosin sowie die Azol-Antimykotika lag die abgelesene MHK bei einer Reduktion des Wachstums um 50\% (MHK 50 ).

Bei einer Reduktion des Wachstums um 50\% wurde ebenfalls die minimale Hemmkonzentration von Caspofungin abgelesen.

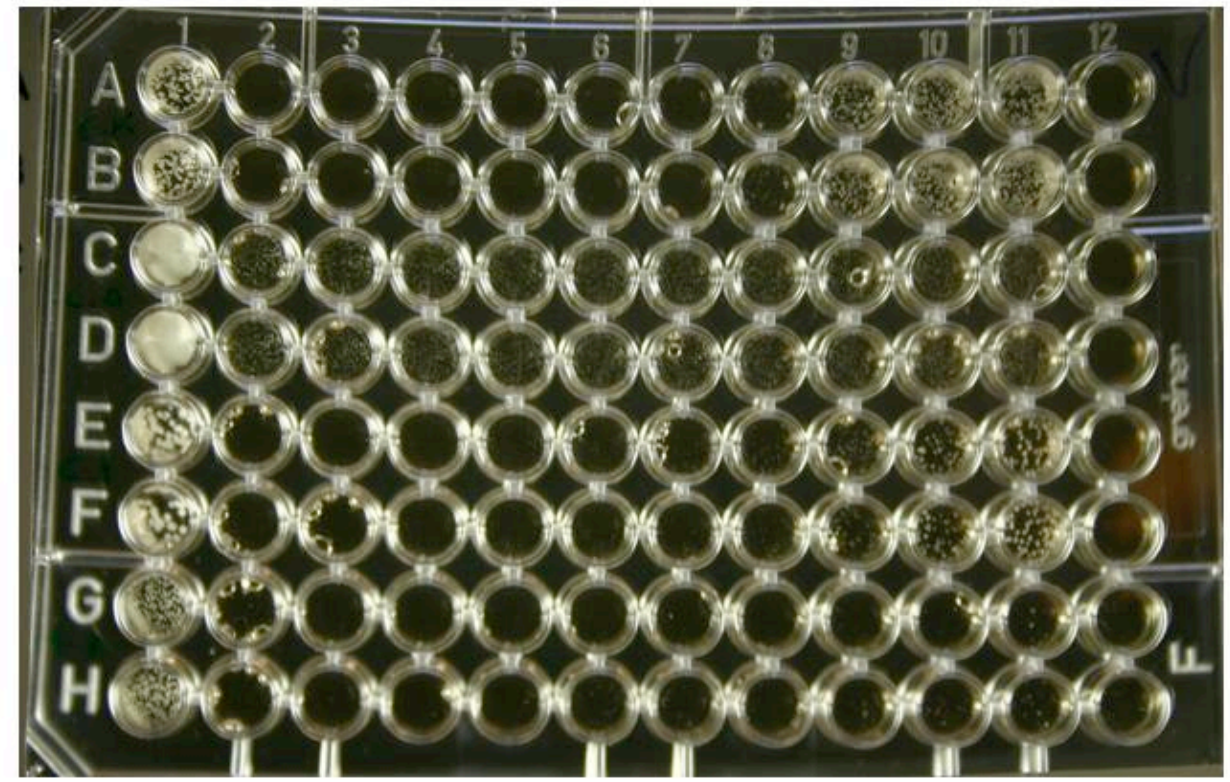

Abbildung 3-2: Mikrodilutionstest der Referenzstämme (in den Reihen A/B ATCC 6258 C. krusei, C/D ATCC 90028 C. albicans, in E/F ATCC 750 C. tropicalis und in G/H ATCC 22019 C. parapsilosis) mit Voriconazol

\subsubsection{Bewertung der Ergebnisse}

Die Ergebnisse der abgelesenen minimalen Hemmkonzentrationen der unterschiedlichen Antimykotika konnten nach den geltenden Referenzbereichen der NCCLS-Richtlinien den sogenannten „breakpoints“, den Kategorien „sensibel“, „dosisabhängig sensibel“ bzw. „intermediär“ sowie „resistent“ zugeordnet werden (NCCLS M27-A2 2002) bzw. aktueller Literatur entnommen 
werden (Pfaller et al. 2011a und 2011b). Des Weiteren wurden die $\mathrm{MHK}_{50}$ sowie $\mathrm{MHK}_{90}$, d.h. die Antimykotika-Konzentrationen, bei denen $50 \%$ bzw. $90 \%$ eine Hemmung in ihrem Wachstum aufwiesen, ermittelt.

\subsubsection{Fluconazol}

Für Fluconazol galten Isolate mit einer $\mathrm{MHK} \leq 8 \mu \mathrm{g} / \mathrm{ml}$ Antimykotikum als sensibel, mit einer MHK zwischen 16 und $32 \mu \mathrm{g} / \mathrm{ml}$ als dosisabhängig sensibel sowie $\geq 64 \mu \mathrm{g} / \mathrm{ml}$ als resistent.

\subsubsection{Itraconazol}

Für Itraconazol wurden Isolate mit einer MHK von $\leq 0,125 \mu \mathrm{g} / \mathrm{ml}$ als sensibel eingestuft, die mit einer MHK zwischen 0,25 und $0,5 \mu \mathrm{g} / \mathrm{ml}$ als dosisabhängig sensibel und die bei einer MHK $1 \mu \mathrm{g} / \mathrm{ml}$ als resistent.

\subsubsection{Voriconazol}

Die Referenzbereiche für Voriconazol, die in der aktuellen Literatur (Pfaller et al. 2006a) Verwendung fanden, lauteten wie folgt: Als sensibel eingeordnet wurden Isolate mit einer $\mathrm{MHK} \leq 1 \mu \mathrm{g} / \mathrm{ml}$, als dosisabhängig sensibel die Stämme mit einer MHK, die bei $2 \mu \mathrm{g} / \mathrm{ml}$ liegt, und als resistent Isolate mit einer $\mathrm{MHK} \geq 4 \mu \mathrm{g} / \mathrm{ml}$.

\subsubsection{Caspofungin}

Für das Antimykotikum Caspofungin lagen speziesspezifisch verschiedene Referenzbereiche vor (Pfaller et al. 2011a). C. albicans, C. tropicalis, C. krusei sowie C. dubliniensis wurden bei einer $M H K \leq 0,25 \mu \mathrm{g} / \mathrm{ml}$ als sensibel eingeordnet, bei $0,5 \mu \mathrm{g} / \mathrm{ml}$ als intermediär und bei $\geq 1 \mu \mathrm{g} / \mathrm{ml}$ als resistent. $C$. glabrata war bei $\leq 0,125 \mu \mathrm{g} / \mathrm{ml}$ sensibel, bei $0,5 \mu \mathrm{g} / \mathrm{ml}$ intermediär und bei $\geq 0,5 \mu \mathrm{g} / \mathrm{ml}$ resistent. Für $C$. parapsilosis und $C$. guillermondii galten die Referenzbereiche $\leq 2 \mu \mathrm{g} / \mathrm{ml}$ (sensibel), $4 \mu \mathrm{g} / \mathrm{ml}$ (intermediär) sowie $8 \mu \mathrm{g} / \mathrm{ml}$ (resistent). Die Isolate von C. Iusitaniae wurden mit einer $\mathrm{MHK} \leq 0,5 \mu \mathrm{g} / \mathrm{ml}$ als sensibel klassifiziert, mit $1 \mu \mathrm{g} / \mathrm{ml}$ als intermediär und mit $\geq 2 \mu \mathrm{g} / \mathrm{ml}$ als resistent. Alle anderen Spezies wurden auf Grundlage der zuvor geltenden Breakpoints mit $\leq 2 \mu \mathrm{g} / \mathrm{ml}$ als sensibel eingestuft (Pfaller et al. 2011a).

\subsubsection{Amphotericin B}

Bei Amphotericin B erfolgte lediglich eine Unterteilung in zwei Gruppen: Isolate mit einer $M H K \leq 1 \mu \mathrm{g} / \mathrm{ml}$ galten als sensibel und die mit einer $\mathrm{MHK} \geq 2 \mu \mathrm{g} / \mathrm{ml}$ als 
resistent.

\subsubsection{5-Fluorcytosin}

Für 5-Fluorcytosin galten Isolate mit einer MHK im Bereich $\leq 4 \mu \mathrm{g} / \mathrm{ml}$ als sensibel, mit einer MHK zwischen 8 und $16 \mu \mathrm{g} / \mathrm{ml}$ als intermediär sowie bei einer MHK $\geq 32 \mu \mathrm{g} / \mathrm{ml}$ als resistent.

Einen Überblick über die unterschiedlichen Konzentrationsbereiche gibt die nachstehende Tabelle 3-1.

Tabelle 3-1: Konzentrationsbereiche zur Bewertung der Wirkung der einzelnen Antimykotika

\begin{tabular}{|c|c|c|c|c|}
\hline Antimykotikum & sensibel & d-sensibel & intermediär & resistent \\
\hline Fluconazol & $\leq 8$ & $16-32$ & & $\geq 64$ \\
\hline Itraconazol & $\leq 0,125$ & $0,25-0,5$ & & $\geq 1$ \\
\hline Voriconazol & $<1$ & 2 & & $\geq 4$ \\
\hline \multicolumn{5}{|l|}{ Caspofungin } \\
\hline C. albicans & $\leq 0,25$ & & 0,5 & $\geq 1$ \\
\hline C. glabrata & $\leq 0,125$ & & 0,25 & $\geq 0,5$ \\
\hline C. parapsilosis & $\leq 2$ & & 4 & $\geq 8$ \\
\hline C. tropicalis & $\leq 0,25$ & & 0,5 & $\geq 1$ \\
\hline C. krusei & $\leq 0,25$ & & 0,5 & $>1$ \\
\hline C. guillermondii & $\leq 2$ & & 4 & $\geq 8$ \\
\hline C. dubliniensis & $\leq 0,25$ & & 0,5 & $\geq 1$ \\
\hline C. Iusitaniae & $\leq 0,5$ & & 1 & $\geq 2$ \\
\hline Andere Spezies & $\leq 2$ & & & $>2$ \\
\hline Amphotericin B & $\leq 1$ & & & $>1$ \\
\hline 5-Fluorcytosin & $\leq 4$ & & 8-16 & $\geq 32$ \\
\hline
\end{tabular}

\subsection{Etest}

Die Verdünnungsstufen des Etests der verschiedenen Antimykotika waren kleinschrittiger und wichen von den Verdünnungsstufen des Mikrodilutionstests 
ab. Die Verdünnungsstufen der Etest-Skala reichen für Fluconazol über 0,016$256 \mu \mathrm{g} / \mathrm{ml}$, für Itraconazol, Voriconazol, Caspofungin, Amphotericin B und 5 -Fluorcytosin über $0,002-32 \mu \mathrm{g} / \mathrm{ml}$.

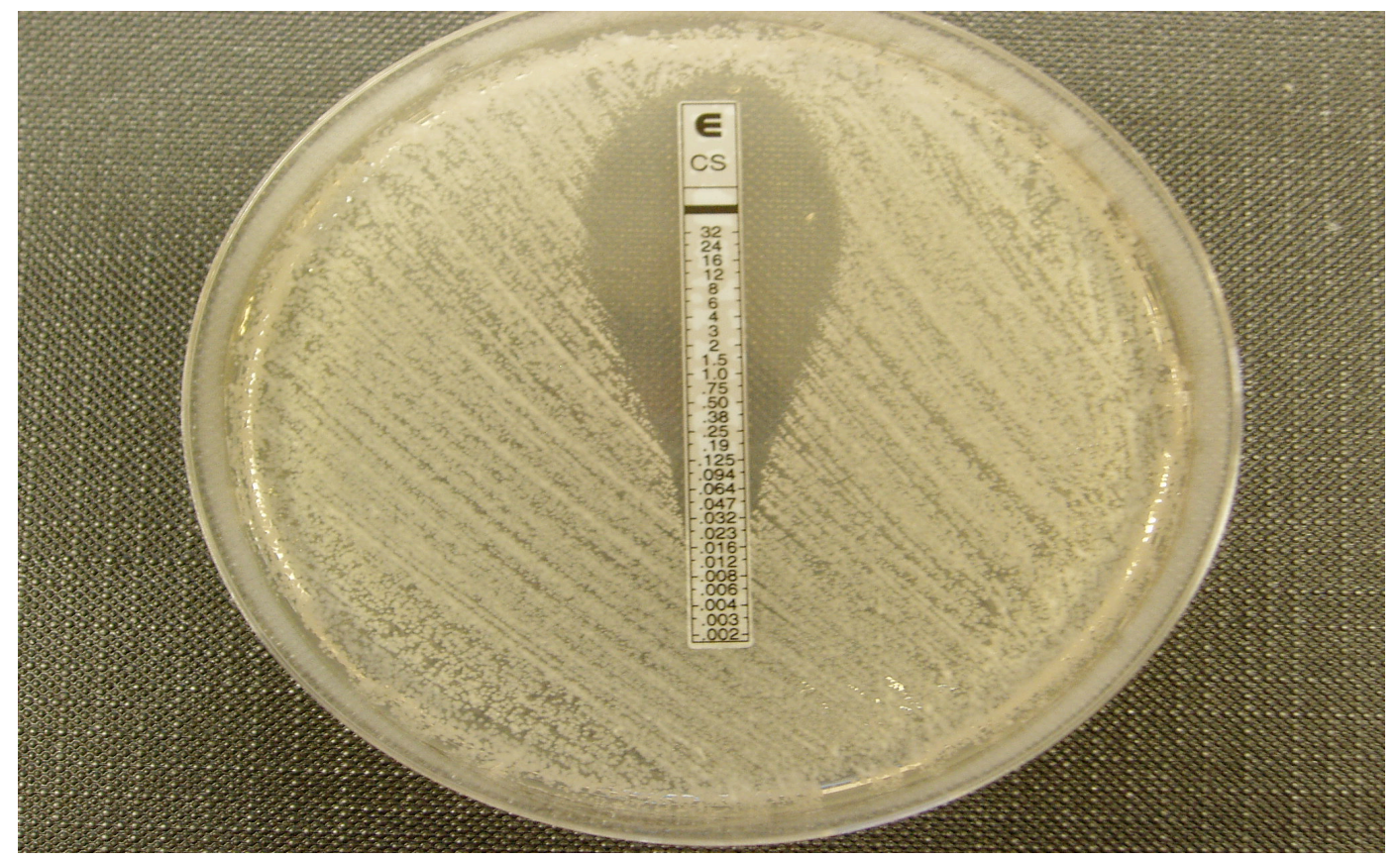

Abbildung 3-3: Etest eines untersuchten Isolates mit Caspofungin

\subsubsection{Präparation und Auftragen des Inokulums}

Die Präparation des Inokulums erfolgte streng nach den Vorgaben des Herstellers ab biodisk. Einzelne Kolonien einer Übernachtreinkultur wurden in steriler Kochsalzlösung gelöst und eine Trübung entsprechend einem StandardMcFarland von 0,5-1 für alle Isolate eingestellt. Das Inokulum wurde, unmittelbar nach der Herstellung zur Beschichtung der für den Etest verwendeten RPMIAgarplatten, verwendet. Dazu wurde ein steriler Wattetupfer in die Suspension eingetaucht und die Agar-Oberfläche vorsichtig und gleichmäßig in drei Richtungen dicht damit benetzt. Das Aufbringen des Etest-Teststreifens erfolgte erst nach vollständigem Trocknen der überschüssigen Flüssigkeit, die teilweise zuvor mit Hilfe einer Pipette abgesaugt werden musste.

\subsubsection{Aufbringen der Etest-Streifen}

Die Etest-Streifen bestanden aus Kunststoff, der für Flüssigkeiten undurchlässig war. Sie waren auf der einen Seite mit einer Skala versehen, die 15 verschiedene 
Verdünnungsstufen anzeigte. Die andere Seite war, der Skalierung folgend, mit der jeweiligen Konzentration des Antimykotikums beschichtet.

Die in Tiefkühlung gelagerten Etest-Streifen mussten zunächst auf Zimmertemperatur auftauen. Auf die getrocknete Oberfläche der Agarplatte wurde der Etest-Streifen dann mit der antimykotisch beschichteten Seite mit Hilfe einer Pinzette aufgebracht. Die Skala mit dem Konzentrationsgradienten lag dabei gut sichtbar oben und der Streifen hatte auf ganzer Länge Kontakt zu der Agarplatte. Lag der Etest-Streifen der Agarplatte einmal auf, so wurde er nicht mehr verschoben oder anders manipuliert. Die Abgabe des Antimykotikums auf die Agarplatte führte in Abhängigkeit von der Antimykotikum-Konzentration und der Sensibilität des Isolates zur Wachstumshemmung.

\subsubsection{Inkubation und Ablesen der Ergebnisse}

Die Inkubation der Testplatten erfolgte für $24-48 \mathrm{~h}$ bei einer Temperatur von $35^{\circ} \mathrm{C}$. Das Ablesen der minimalen Hemmkonzentration erfolgte dann visuell anhand der Etest-Skala. Abgelesen wurde an der Stelle der Skala, an der die Konzentration des Antimykotikums gerade noch ausreichte, um das Wachstum der Hefen zu verhindern, d.h. am unteren Pol der Ellipse. Bei grenzwertigen Ergebnissen war laut Hersteller der höhere Wert abzulesen (AB Biodisk Etest Arbeitsanleitung 3b 2000).

\subsubsection{Bewertung der Ergebnisse}

Die abgelesenen Etest-Ergebnisse der MHK wurden nach den vom Hersteller veröffentlichten Referenzbereichen, den „breakpoints“, bewertet. Diese entsprachen den für die Mikrodilution publizierten Bereichen (NCCLS M27-A2 2002; vgl. Abschnitt 3.4.8 und Tabelle 3-1). Außerdem wurden $\mathrm{MHK}_{50}$ sowie $\mathrm{MHK}_{90}$ bestimmt. 


\subsection{Qualitätskontrolle}

Zur Qualitätskontrolle des Mikrodilutionstests sowie des Etests wurden bei jeder Testung Referenzstämme mitgeführt. Hierzu wurden die zwei Referenzstämme C. parapsilosis ATCC 22019 sowie C. krusei ATCC 6258 verwendet. Die gemessene MHK dieser Isolate wurde nach jeder Testung mit den in Tabelle 3-2 und Tabelle 3-3 aufgeführten Referenzergebnissen verglichen, so dass eine gleichmäßige Inkubation unter stabilen Bedingungen gewährleistet war (Barry et al. 2000, NCCLS M27-A2 2002, Pfaller et al. 1996).

\subsubsection{Mikrodilution}

Des Weiteren fand zur Sicherung der gleichbleibenden Qualität des Mikrodilutionstests die Testung im Doppelansatz statt. Jedes Isolat wurde in zwei Reihen getestet, und erst nach Bildung des Mittelwertes wurde die MHK ermittelt. Eine weitere Maßnahme zur Qualitätssicherung stellte die regelmäßige stichprobenartige Kontrolle der Einsaat dar (vgl. Abschnitt 3.4.2).

Tabelle 3-2: Referenzbereiche der zwei verwendeten Isolate ATCC 6258 (C. krusei) und ATCC 22019 (C. parapsilosis), nach NCCLS M27-A2 2002

\begin{tabular}{|c|c|c|c|c|}
\hline \multirow[b]{2}{*}{ Antikmykotikum } & \multicolumn{2}{|c|}{ ATCC 6258 (C. krusei) } & \multicolumn{2}{|c|}{ ATCC 22019 (C. parapsilosis) } \\
\hline & $24 \mathrm{~h}$ & $48 \mathrm{~h}$ & $24 \mathrm{~h}$ & $48 \mathrm{~h}$ \\
\hline Fluconazol & $8-64$ & $16-128$ & $0,5-4$ & $1-4$ \\
\hline Itraconazol & $0,125-1$ & $0,25-1$ & $0,125-0,5$ & $0,125-0,5$ \\
\hline Voriconazol & $0,0625-0,5$ & $0,125-1$ & $0,0625-0,125$ & $0,0313-0,25$ \\
\hline Caspofungin & $0,125-1$ & $0,25-1$ & $0,25-1$ & $0,5-4$ \\
\hline Amphotericin B & $0,5-2$ & $1-4$ & $0,25-2$ & $0,5-4$ \\
\hline 5-Fluorcytosin & $4-16$ & $8-32$ & $0,0625-0,25$ & $0,125-0,5$ \\
\hline
\end{tabular}

\subsubsection{Etest}

Im Folgenden sind in Tabelle 3-3 die Ergebnisse für die Testung der Referenzstämme anhand des Etests abgebildet. 
Tabelle 3-3: Referenzbereiche der zwei verwendeten Isolate ATCC 6258 (C. krusei) und ATCC 22019 (C. parapsilosis). Die Angaben stammten vom Hersteller des Etests ab biodisk (EAS 006); * keine Empfehlung für Fluconazol und 5-Fluorcytosin

\begin{tabular}{|l|c|c|}
\hline & $\begin{array}{c}\text { ATCC } \mathbf{6 2 5 8} \text { (C. krusei) } \\
\text { Antimykotikum }\end{array}$ & $\begin{array}{c}\text { ATCC 22019 (C. parapsilosis) } \\
\mathbf{2 4} \mathbf{~ h}\end{array}$ \\
\hline Fluconazol & - & $1,0-8,0$ \\
\hline Itraconazol & $0,25-1,0$ & $0,064-0,25$ \\
\hline Voriconazol & $0,25-1,0$ & $0,016-0,064$ \\
\hline Caspofungin & $0,25-1,0$ & $0,25-2,0$ \\
\hline Amphotericin B & $0,5-2,0$ & $0,25-1,0$ \\
\hline 5-Fluorcytosin & - & $0,064-0,25$ \\
\hline
\end{tabular}

\subsection{Korrelation der Testmethoden Mikrodilution und Etest}

Die Korrelation der Ergebnisse der beiden Testmethoden erfolgte nach zwei unterschiedlichen Gesichtspunkten.

Zum Ersten wurden die numerischen Werte der MHKs der Testmethoden verglichen. Als übereinstimmend bezüglich der Konzentrationsstufen wurden solche Ergebnisse bezeichnet, die maximal zwei Konzentrationsstufen ober- bzw. unterhalb des verglichenen Ergebnisses lagen.

Zum Zweiten wurde die Übereinstimmung der Kategorien anhand der oben beschriebenen Breakpoints geprüft (vgl. Abschnitt 3.4.8 und 3.5.4). Als übereinstimmende Ergebnisse bezüglich der Kategorie galten somit Ergebnisse, die durch beide Testverfahren die gleiche Zuordnung zu den Kategorien „sensibel“, „dosisabhängig sensibel“ bzw. „intermediär“ und „resistent“ erfuhren. 


\section{$4 \quad$ Ergebnisse}

\subsection{Epidemiologischer Teil}

\subsubsection{Teilnehmende Institutionen}

Insgesamt nahmen 37 unterschiedliche Institutionen aus dem ganzen Bundesgebiet teil. Dies waren 15 Universitätskliniken, sechs mittlere bis große Krankenhäuser, sechs staatliche Institutionen sowie zehn Laborpraxisgemeinschaften.

Die Einrichtungen sind in Tabelle 4-1 gemeinsam mit Angaben über die Anzahl der eingesendeten Isolate aufgeführt. Im Anhang sind alle Einsender mit Adressen gelistet.

Die geographische Verteilung der Institutionen innerhalb Deutschlands verdeutlicht die Abbildung 4-1. Sie zeigt, dass die Institutionen im gesamten Bundesgebiet so verteilt lagen, dass eine breitflächige Datenerhebung gegeben war.

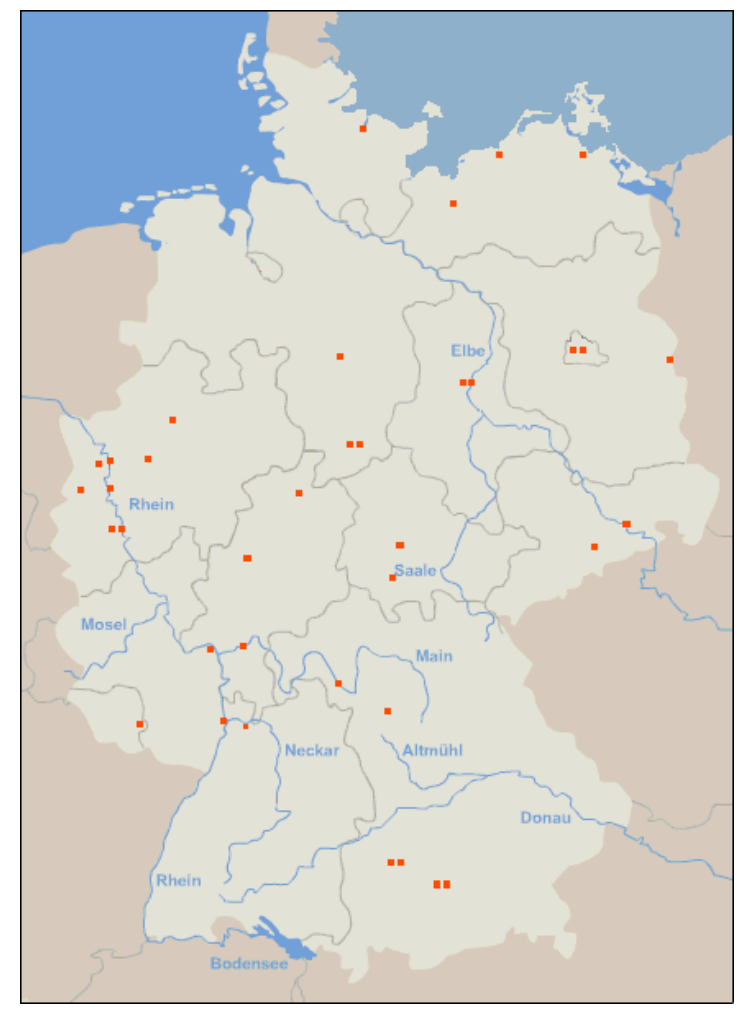

Abbildung 4-1: Verteilung der Einsender innerhalb Deutschlands 
Tabelle 4-1: Auflistung aller Einsender

\begin{tabular}{|c|c|c|}
\hline Einsender & Ort & $\mathbf{N}$ \\
\hline Inst. für Infektionsmedizin, Campus Benjamin Franklin, Charité & Berlin & 81 \\
\hline Inst. für Klinische Chemie und Labormedizin, Krankenhaus Dresden-Friedrichstadt & Dresden & 68 \\
\hline Inst. für Med. Mikrobiologie, Universitätsklinikum Mannheim & Mannheim & 45 \\
\hline Inst. für Med. Mikrobiologie, Universitätsklinikum Münster & Münster & 41 \\
\hline Inst. für Med. Mikrobiologie und Hygiene, Universität Mainz & Mainz & 35 \\
\hline Inst. für Med. Mikrobiologie, Universitätsklinikum Göttingen & Göttingen & 33 \\
\hline Inst. für Mikrobiologie und Hygiene, Universitätsklinikum des Saarlandes & Homburg / Saar & 30 \\
\hline Niedersächsisches Landesgesundheitsamt & Hannover & 29 \\
\hline Inst. für Med. Mikrobiologie, Virologie und Hygiene, Universitätsklinikum Rostock & Rostock & 25 \\
\hline Gemeinschaftspraxis Stein, Kehren, Beckers & Mönchengladbach & 21 \\
\hline Inst. für Hygiene und Laboratoriumsmedizin, Städtische Krankenhäuser Krefeld & Krefeld & 15 \\
\hline Ärztliches Labor Becker, Olgemöller \& Kollegen & München & 14 \\
\hline Inst. für Laboratoriumsmedizin, Helios Kliniken Schwerin & Schwerin & 14 \\
\hline Inst. für Med. Mikrobiologie, Universität Magdeburg & Magdeburg & 13 \\
\hline Friedrich-Loeffler-Inst. für Med. Mikrobiologie, Universität Greifswald & Greifswald & 12 \\
\hline Max von Pettenkofer-Inst. für Hygiene und Med. Mikrobiologie, Universität München & München & 12 \\
\hline Inst. für Med. Mikrobiologie und Virologie, Universitätsklinikum Schleswig-Holstein & Kiel & 11 \\
\hline Inst. für Laboratoriumsmedizin, Städtische Kliniken & Frankfurt (Main) & 11 \\
\hline Landesamt für Verbraucherschutz des Landes Sachsen-Anhalt & Magdeburg & 6 \\
\hline Inst. für Med. Mikrobiologie, Universitätsklinikum Düsseldorf & Düsseldorf & 5 \\
\hline Gemeinschaftspraxis für Laboratoriumsmedizin, Hülsmann, Baczko und Becker & Kassel & 5 \\
\hline Robert-Koch-Institut & Berlin & 5 \\
\hline Inst. für Med. Mikrobiologie, Medizinaluntersuchungsamt, Stadt Bochum & Bochum & 5 \\
\hline Landesuntersuchungsanstalt für Gesundheits- und Veterinärwesen Sachsen & Chemnitz & 3 \\
\hline Inst. für Med. Mikrobiologie und Krankenhaushygiene, Uniklinikum Marburg & Marburg & 3 \\
\hline Zentrallabor, Wedau Kliniken, Klinikum Duisburg & Duisburg & 3 \\
\hline Thüringer Landesamt für Lebensmittelsicherheit und Verbraucherschutz, Erfurt & Erfurt & 3 \\
\hline Ärztliches Labor Berthold und Kollegen & Frankfurt (Oder) & 3 \\
\hline Ärztliches Labor Wimmer und Partner & Augsburg & 3 \\
\hline Inst. für Med. Mikrobiologie, Immunologie und Hygiene, Uniklinikum Köln & Köln & 2 \\
\hline Ärztliches Labor Schottdorf und Blanke & Augsburg & 4 \\
\hline Ärztliches Labor Wagner und Partner & Göttingen & 2 \\
\hline Ärztliches Labor Wisplinghoff, Spieckermann und Co & Köln & 1 \\
\hline Ärztliches Labor Runnebaum und Partner & Eppelheim & 1 \\
\hline Ärztliches Labor Arnold & Würzburg & 1 \\
\hline Inst. für Klinische Mikrobiologie, Immunologie und Hygiene, Universität Erlangen-Nürnberg & Erlangen & 1 \\
\hline Inst. für Klinische Chemie und Laboratoriumsmedizin, Zentralklinikum Suhl & Suhl & 1 \\
\hline
\end{tabular}




\subsubsection{Klinische Angaben}

Die Angaben der Begleitscheine der Isolate (Abbildung 2-1) waren unvollständig, so dass für die Auswertung die Daten zu 394 Proben vorlagen. Der größte Teil (230 Proben) stammte von Patienten, die intensivmedizinisch behandelt wurden $(40,6 \%)$. Mit 91 Proben wurde die zweitgrößte Gruppe durch die internistisch versorgten Patienten gebildet (16\%). In der chirurgischen Abteilung wurden 43 Patienten $(7,6 \%)$ behandelt. $5,3 \%$ der Patienten wurden in den anderen Fachbereichen (Gynäkologie, Neurologie, Pädiatrie und Urologie) betreut (30 Proben). Bei 173 Proben (30,5\%) fehlten die klinischen Angaben (Abbildung 4-2). Die Behandlung wurde bei vier Patienten ambulant durchgeführt; alle anderen befanden sich in stationärer Behandlung.

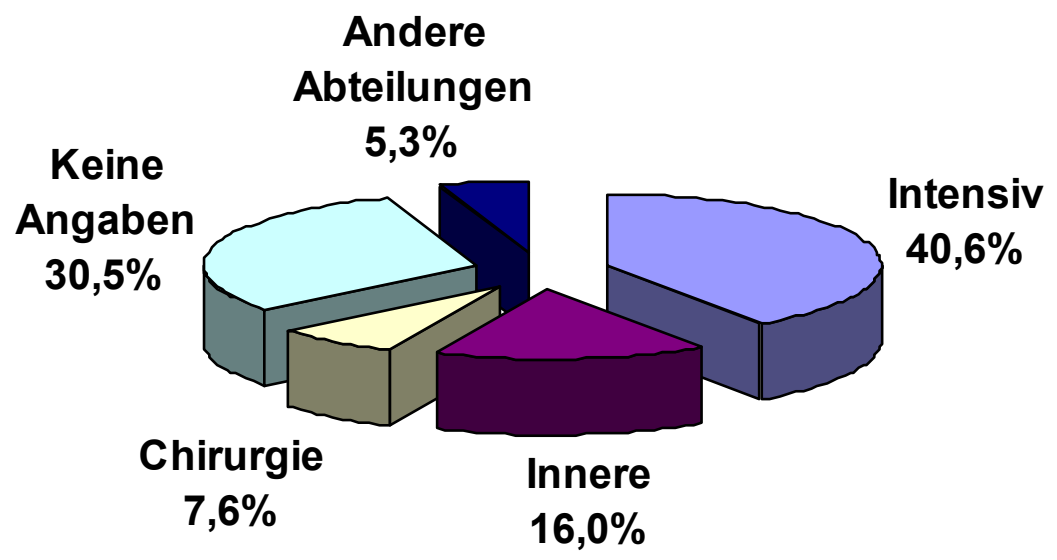

Abbildung 4-2: Verteilung der behandelnden Fachbereiche

\subsubsection{Speziesverteilung der Isolate in allen Materialien}

Die 567 eingeschickten Isolate gehörten 21 unterschiedlichen Spezies an (Tabelle 4-2). C. albicans machte den weitaus größten Anteil aus (322 Stämme, 56,8\%). Die zweitgrößte Gruppe bildete C. glabrata (105 Isolate, 18,5\%). C. parapsilosis folgte mit einem Anteil von $8,1 \%$ (46 Isolate) während C. tropicalis 
bei 7,4\% lag (42 Stämme). C. kefyr und C. krusei waren durch $11(1,9 \%)$ bzw. acht Isolate $(1,4 \%)$ vertreten. Von den Spezies $\mathrm{Cr}$. neoformans sowie $S$. cerevisiae lagen jeweils fünf Isolate vor (je 0,9\%). Die restlichen 23 Isolate $(4,1 \%)$ gehörten zu den selteneren Candida-Spezies und verteilten sich wie folgt: Vier Isolate $(0,7 \%)$ zählten zu C. guilliermondii, drei $(0,5 \%)$ zu C. dubliniensis und eines $(0,2 \%)$ zu G. capitatum, drei Stämme zu C. inconspicua $(0,5 \%)$, jeweils zwei Isolate zu C. Iusitaniae, C. norvegensis und Trichosporon vor (je 0,4\%) sowie jeweils ein Isolat zu C. catenulata, C. colliculosa, C. famata, C. intermedia, C. rugosa und C. utilis (je 0,2\%). Der Anteil der Non-albicans-Stämme lag mit 245 Stämmen bei 43,2\%. In zwei Fällen lagen gemischte Infektionen durch zwei verschiedene Candida-Spezies vor (C. albicans und C. glabrata sowie durch $C$. albicans und C. krusei). Die Isolate wurden separat getestet und ausgewertet.

Tabelle 4-2: Speziesverteilung der 567 Isolate in allen Materialien

\begin{tabular}{|l|c|c|}
\hline \multicolumn{1}{|c|}{ Spezies } & Anzahl & Anteil in Prozent \\
\hline C. albicans & 322 & 56,8 \\
\hline C. glabrata & 105 & 18,5 \\
\hline C. parapsilosis & 46 & 8,1 \\
\hline C. tropicalis & 42 & 7,4 \\
\hline C. kefyr & 11 & 1,9 \\
\hline C. krusei & 8 & 1,4 \\
\hline C. guilliermondii & 4 & 0,7 \\
\hline C. dubliniensis & 3 & 0,5 \\
\hline C. inconspicua & 3 & 0,5 \\
\hline C. lusitaniae & 2 & 0,3 \\
\hline C. norvegensis & 2 & 0,3 \\
\hline C. catenulata & 1 & 0,2 \\
\hline C. colliculosa & 1 & 0,2 \\
\hline C. famata & 1 & 0,2 \\
\hline C. intermedia & 1 & 0,2 \\
\hline C. rugosa & 1 & 0,2 \\
\hline C. utilis & 1 & 0,2 \\
\hline Cr. neoformans & 5 & 0,9 \\
\hline Geotrichum capitatum & 1 & 0,2 \\
\hline S. cerevisiae & 5 & 0,9 \\
\hline Trichosporon spp. & 2 & 0,3 \\
\hline
\end{tabular}




\subsubsection{Speziesverteilung in Bezug auf Geschlecht und Alter}

\subsubsection{Geschlecht}

Bei einem Isolat fehlte die Angabe zum Geschlecht. 310 Isolate (54,8\%) stammten von männlichen und 256 Isolate $(45,2 \%)$ von weiblichen Patienten. Das Verhältnis männlicher zu weiblicher Patienten lag somit bei $310: 256$ bzw. $1: 0,8$. Weibliche Patienten überwogen nur in den frühen sowie in den späten Lebensjahren.

Für C. albicans, C. glabrata und C. tropicalis lag der Anteil männlicher Patienten zwischen 54 bis 59\%. Alle Cr. neoformans-Isolate waren von männlichen Patienten. Die Isolate weiblicher Patienten überwogen bei den Spezies $C$. parapsilosis (67\%), C. kefyr (100\%) und G. capitatum (100\%).

\subsubsection{Alter}

Auch bezüglich des Alters fehlten bei einem Isolat die Angaben. Der älteste in die Studie einbezogene Patient war ein männlicher Patient, der im Jahre 1906 geboren wurde. Der jüngste Patient war ein im Jahr 2005 geborener weiblicher Säugling. 14 Isolate $(2,5 \%)$ stammten von Säuglingen und Kleinkindern (20002005), wobei es sich zur Hälfte um männliche Patienten handelte. Demgegenüber standen 275 Proben von über 65-jährigen Patienten. Das Durchschnittsalter lag bei 60,6 Jahren. Damit waren über zwei Drittel der Patienten über 55 Jahre alt.

Die Abbildung 4-3 zeigt das Vorkommen der Spezies C. albicans, C. glabrata, C. parapsilosis und $C$. tropicalis in acht verschiedenen Altersgruppen. $C$. parapsilosis dominierte in der Gruppe der unter 5-jährigen Patienten deutlich $(35,7 \%)$, spielte als Pathogen jedoch auch im höheren Lebensalter (66- bis 75 Jährige) wieder eine wichtige Rolle. C. albicans war in allen anderen Altersgruppen als Haupterreger vertreten. Des Weiteren wird deutlich, dass $C$. glabrata mit zunehmendem Alter der Patienten häufiger vorkommt.

In der Tabelle 7-1 (S. 95) ist die Speziesverteilung der Isolate in den verschiedenen Altersgruppen für männliche und weibliche Patienten dargestellt. 


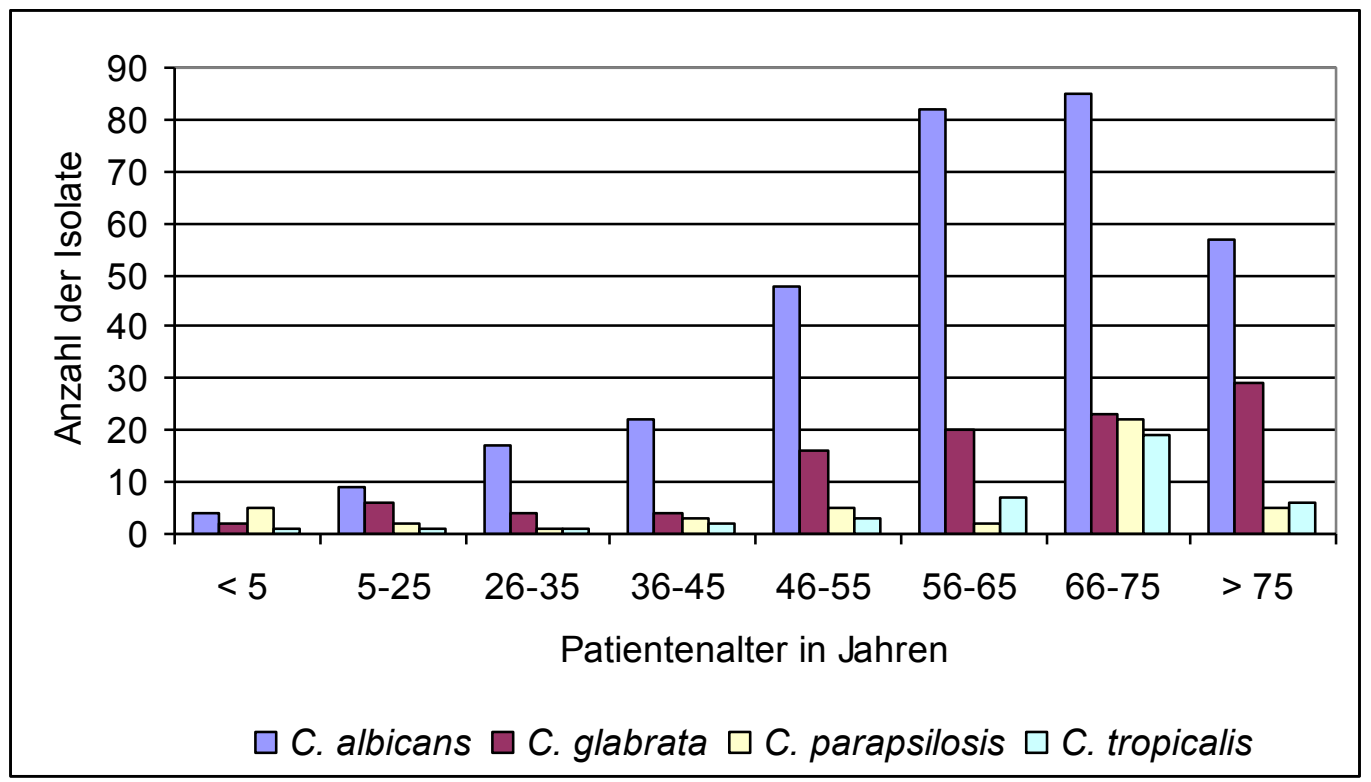

Abbildung 4-3: Vorkommen ausgewählter Candida spp. in den verschiedenen Altersgruppen

\subsubsection{Speziesverteilung der Isolate in den einzelnen Materialien}

Alle Isolate stammten aus primär sterilen Materialien (Tabelle 4-3). 438 der Isolate $(77,2 \%)$ waren aus Blutkulturen, 69 Isolate $(12,2 \%)$ aus Proben des Brustund Bauchraumes. 23 Proben $(4,1 \%)$ wurden aus ZVK-Spitzen angezüchtet. 22 Isolate $(3,9 \%)$ stammten aus Punktaten und Abstrichen anderer Körperregionen (zur näheren Definition s.u.). Neun der Isolate wurden aus Liquor-Proben gewonnen (1,6\%); die restlichen sechs Isolate aus Bronchiallavagen $(1,1 \%)$.

Tabelle 4-3: Verteilung der insgesamt 567 Isolate in Bezug auf die unterschiedlichen primär sterilen Materialien

\begin{tabular}{|l|c|c|}
\hline \multicolumn{1}{|c|}{ Material } & Anzahl & Anteil in Prozent \\
\hline Blutkulturen & 438 & 77,2 \\
\hline Proben aus Brust- und Bauchraum & 69 & 12,2 \\
\hline ZVK-Spitzen & 23 & 4,1 \\
\hline $\begin{array}{l}\text { Punktate und Proben } \\
\text { anderer Körperregionen }\end{array}$ & 22 & 3,9 \\
\hline Liquor & 9 & 1,6 \\
\hline Bronchiallavage & 6 & 1,1 \\
\hline
\end{tabular}




\subsubsection{Speziesverteilung in den Proben aus Blutkulturen}

Insgesamt wurden aus den 438 Blutkulturen 20 verschiedene Spezies differenziert. Der Anteil der Non-albicans-Stämme lag bei 43,1\%. C. albicans war mit 249 Isolaten die häufigste Spezies. Es lagen 79 C. glabrata-Stämme vor. C. parapsilosis und $C$. tropicalis rangierten mit 41 und 27 Isolaten auf dem dritten und vierten Platz.

\subsubsection{Speziesverteilung in den Proben aus Liquor}

Insgesamt neun Isolate stammten aus Liquor. Aus innen wurden vier unterschiedliche Spezies isoliert. Der Anteil der Non-albicans-Stämme lag bei $55,6 \%$. Mit vier Stämmen lag C. albicans am häufigsten vor. Es folgten $C$. glabrata und Cr. neoformans mit jeweils zwei Isolaten. C. tropicalis war mit einem Isolat vertreten. Es stammten zwei von insgesamt fünf vorkommenden $\mathrm{Cr}$. neoformans-Isolaten (40\%) aus den Liquor-Proben.

\subsubsection{Speziesverteilung in den Proben aus ZVK-Spitzen}

Aus den 23 ZVK-Proben wurden vier verschiedene Spezies differenziert. Der Anteil der Non-albicans-Isolate lag bei $27,8 \%$ und war damit niedriger als bei den anderen Materialien. Es lagen 18 C. albicans-Isolate, jeweils zwei C. glabrataund C. parapsilosis-Isolate sowie ein C. kefyr-Stamm vor.

\subsubsection{Speziesverteilung in den Proben aus Brust- und Bauchraum}

69 Isolate stammten aus Abstrichen, Punktaten und Biopsien, die aus Brust- und Bauchraum gewonnen wurden. Dazu zählten u.a. Aszites- und Pleurapunktate, Leberbiopsien, intraoperative Abstriche, Magensaftproben sowie Gallenblasenpunktate. Es wurden sieben Spezies isoliert. Der Non-albicansAnteil lag bei $55,1 \%$. Neben 31 C. albicans-Isolaten lagen 14 C. tropicalis-Isolate, zwei Stämme der Spezies C. norvegensis sowie jeweils ein Isolat von C. kefyr, $C$. krusei und C. Iusitaniae vor.

\subsubsection{Speziesverteilung in den Proben aus Bronchiallavagen}

Die sechs aus Bronchiallavagen stammenden Isolate gehörten der Spezies $C$. albicans an. Eine Kontamination ist aufgrund der Technik bei der Gewinnung der Proben möglich. In dieser Arbeit werden die Proben, die aus den Bronchiallavagen stammten, jedoch als primär steril angesehen. 


\subsubsection{Speziesverteilung in den Proben übriger Körperregionen}

22 Isolate waren aus Punktaten und Abstrichen anderer Körperregionen. Hierzu zählten verschiedene Abstriche (vordere Augenkammer, Hüftimplantat, Wundabstriche, intraoperative Abstriche), die Spitze eines Intubationstubus, sowie Punktate, z. B. primär steriler Urin von suprapubischen Blasenkathetern. Der Non-albicans-Anteil lag bei $57,1 \%$. Es lagen 14 C. albicans-Stämme, fünf $C$. glabrata- und zwei C. guilliermondii -Isolate sowie ein C. parapsilosis-Isolat vor.

Die Speziesverteilung in den einzelnen Materialien ist in Tabelle 4-4 dargestellt.

Tabelle 4-4: Verteilung der Spezies in den Untersuchungsmaterialien. Dargestellt sind die isolierten Spezies aus Blutkulturen, Liquor und Zentralen Venenkathetern (ZVK), Abstrichen aus Brust- und Bauchraum, Bronchiallavage (BAL) sowie zusammengefasst aus übrigen Materialien. Zum Vergleich ist die Gesamtheit der Materialien der jeweiligen Spezies (Gesamt) aufgelistet.

\begin{tabular}{|c|c|c|c|c|c|c|c|}
\hline & \multicolumn{7}{|c|}{ Anzahl der Isolate aus dem jeweiligen Material (\%) } \\
\hline & $\begin{array}{c}\text { Blut- } \\
\text { kulturen }\end{array}$ & Liquor & ZVK & $\begin{array}{c}\text { Proben aus Brust- } \\
\text { und Bauchraum }\end{array}$ & BAL & \begin{tabular}{|c|} 
Übrige \\
Materialien \\
\end{tabular} & Gesamt \\
\hline C. albicans & 249 & 4 & 18 & 31 & 6 & 14 & 322 \\
\hline C. glabrata & 79 & & 2 & 19 & & 5 & 105 \\
\hline C. parapsilosis & 41 & 2 & 2 & & & 1 & 46 \\
\hline C. tropicalis & 27 & 1 & & 14 & & & 42 \\
\hline C. kefyr & 9 & & 1 & 1 & & & 11 \\
\hline C. krusei & 7 & & & 1 & & & 8 \\
\hline C. guilliermondii & 2 & & & & & 2 & 4 \\
\hline C. dubliniensis & 3 & & & & & & 3 \\
\hline C. inconspicua & 3 & & & & & & 3 \\
\hline C. Iusitaniae & 1 & & & 1 & & & 2 \\
\hline C. norvegensis & & & & 2 & & & 2 \\
\hline C. catenulata & 1 & & & & & & 1 \\
\hline C. colliculosa & 1 & & & & & & 1 \\
\hline C. famata & 1 & & & & & & 1 \\
\hline C. intermedia & 1 & & & & & & 1 \\
\hline C. rugosa & 1 & & & & & & 1 \\
\hline C. utilis & 1 & & & & & & 1 \\
\hline Cr. neoformans & 3 & 2 & & & & & 5 \\
\hline G. capitatum & 1 & & & & & & 1 \\
\hline S. cerevisiae & 5 & & & & & & 5 \\
\hline Trichosporon spp. & 2 & & & & & & 2 \\
\hline Anzahl der Isolate & 438 & 9 & 23 & 69 & 6 & 22 & 567 \\
\hline Anzahl der Spezies & 20 & 4 & 4 & 7 & 1 & 4 & 21 \\
\hline$\%$ Non-albicans-Isolate & 42,7 & 55,6 & 27,8 & 55,1 & & 57,1 & 43,2 \\
\hline
\end{tabular}




\subsection{Ergebnisse des Mikrodilutionstests}

Die Ergebnisse der Mikrodilutions-Testung werden für die einzelnen Antimykotika separat abgehandelt. Die 567 Isolate wurden durch Mikrodilution gegenüber Fluconazol, Itraconazol, Voriconazol Caspofungin, Amphotericin B sowie 5-Fluorcytosin getestet.

\subsubsection{Fluconazol}

\subsubsection{C. albicans}

96,6\% (311 Isolate) der C. albicans-Isolate waren Fluconazol-empfindlich. 0,6\% (zwei Isolate) wiesen eine dosisabhängige Fluconazol-Empfindlichkeit auf, 2,8\% (neun Isolate) waren resistent. Die minimalen Hemmkonzentrationen lagen im Bereich von $<0,25 \mu \mathrm{g} / \mathrm{ml}$ bis $>32 \mu \mathrm{g} / \mathrm{ml}$. $\mathrm{MHK}_{50}$ sowie $\mathrm{MHK}_{90}$ betrugen $0,25 \mu \mathrm{g} / \mathrm{ml}$ und $1 \mu \mathrm{g} / \mathrm{ml}$ (Tabelle 4-5).

\subsubsection{C. glabrata}

Die C. glabrata-Isolate wiesen zu 80\% eine Fluconazol-Empfindlichkeit auf (84 Isolate waren empfindlich). 14,3\% (15 Stämme) waren dosisabhängig empfindlich und $5,7 \%$ (sechs Stämme) waren resistent. Die MHKs lagen im Bereich zwischen $<0,25 \mu \mathrm{g} / \mathrm{ml}$ und $>32 \mu \mathrm{g} / \mathrm{ml}$. Die $\mathrm{MHK}_{50}$ und $\mathrm{MHK}_{90}$ wurden bei $4 \mu \mathrm{g} / \mathrm{ml}$ sowie $8 \mu \mathrm{g} / \mathrm{ml}$ berechnet (Tabelle $4-5$ ).

\subsubsection{C. parapsilosis}

95,6\% (44 Isolate) der Spezies C. parapsilosis konnten als empfindlich eingeordnet werden. 4,4\% (zwei Isolate) waren dosisabhängig empfindlich. Es traten keine Resistenzen auf. Die MHKs lagen im Bereich zwischen $<0,25 \mu \mathrm{g} / \mathrm{ml}$ und $32 \mu \mathrm{g} / \mathrm{ml}$. Die $\mathrm{MHK}_{50}$ betrug $0,5 \mu \mathrm{g} / \mathrm{ml}$ und die $\mathrm{MHK}_{90} 2 \mu \mathrm{g} / \mathrm{ml}$ (Tabelle 4-5).

\subsubsection{C. tropicalis}

Die C. tropicalis-Isolate zeigten folgende Testergebnisse: 92,8\% (39 Isolate) waren empfindlich, 2,4\% (ein Isolat) dosisabhängig empfindlich, und 4,8\% (zwei Isolate) waren resistent. Die MHKs lagen innerhalb des Bereiches von 0,25 $\mathrm{gg} / \mathrm{ml}$ bis $>32 \mu \mathrm{g} / \mathrm{ml}$. $\mathrm{MHK}_{50}$ und $\mathrm{MHK}_{90}$ wurden mit $0,5 \mu \mathrm{g} / \mathrm{ml}$ sowie $2 \mu \mathrm{g} / \mathrm{ml}$ ermittelt (Tabelle 4-5). 


\subsubsection{C. kefyr}

Alle C. kefyr-Stämme waren empfindlich gegenüber Fluconazol. Die MHKs lagen im Bereich von $0,25 \mu \mathrm{g} / \mathrm{ml}$ bis $0,5 \mu \mathrm{g} / \mathrm{ml}$. $\mathrm{MHK}_{50}$ und $\mathrm{MHK}_{90}$ wurden bei jeweils $0,5 \mu \mathrm{g} / \mathrm{ml}$ ermittelt (Tabelle 4-5).

\subsubsection{C. krusei}

Nur ein Isolat (12,5\%) war empfindlich, lag aber mit einer MHK von $8 \mu \mathrm{g} / \mathrm{ml}$ an der oberen Grenze des empfindlichen Bereichs. 62,5\% (fünf Isolate) wiesen eine dosisabhängige Empfindlichkeit auf und 25\% (zwei Isolate) waren resistent. Die MHKs lagen im Bereich der Konzentrationsstufen von $8 \mu \mathrm{g} / \mathrm{ml}$ bis $>32 \mu \mathrm{g} / \mathrm{ml}$. Die $\mathrm{MHK}_{50}$ betrug $16 \mu \mathrm{g} / \mathrm{ml}$, die $\mathrm{MHK}_{90}>32 \mu \mathrm{g} / \mathrm{ml}$ (Tabelle 4-5).

\subsubsection{Andere Spezies}

Drei der S. cerevisiae-Isolate waren sensibel und zwei dosisabhängig sensibel. Drei sensible $C$. guilliermondii-Isolate standen einem dosisabhängig sensiblen Isolat gegenüber. Die drei $C$. inconspicua-Stämme waren dosisabhängig sensibel. Eines der zwei $C$. norvegensis-Isolate war sensibel, das andere Isolat war resistent. Das G. capitatum-Isolat war dosisabhängig sensibel. Die Isolate der weiteren Spezies waren durchgängig sensibel. Die MHKs lagen im Bereich von $<0,25 \mu \mathrm{g} / \mathrm{ml}$ bis $>32 \mu \mathrm{g} / \mathrm{ml}$ (Tabelle $7-2$, S. 97 ).

Die genaue Verteilung der Isolate über die unterschiedlichen Konzentrationsstufen ist Tabelle 7-2 (S. 97) zu entnehmen.

Tabelle 4-5: $\mathrm{MHK}_{50}, \mathrm{MHK}_{90}$ und die Bereiche der minimalen Hemmkonzentrationen (in $\mu \mathrm{g} / \mathrm{ml}$ ) sowie die Empfindlichkeit der häufigsten Spezies gegenüber Fluconazol

\begin{tabular}{|c|c|c|c|c|c|c|c|}
\hline \multirow[b]{2}{*}{ Fluconazol } & \multirow[b]{2}{*}{$\mathbf{N}$} & \multirow[b]{2}{*}{ MHK $_{50}$} & \multirow[b]{2}{*}{$\mathrm{MHK}_{90}$} & \multirow{2}{*}{$\begin{array}{c}\text { Bereich } \\
\text { der MHKs }\end{array}$} & \multicolumn{3}{|c|}{ N (\%) der Isolate } \\
\hline & & & & & sensibel & d-sensibel & resistent \\
\hline Gesamt & 567 & 0,5 & 8 & $<0,25->32$ & $515(90,8)$ & $32(5,6)$ & $20(3,6)$ \\
\hline C. albicans & 322 & 0,3 & 1 & $<0,25->32$ & $311(96,6)$ & $2(0,6)$ & $9(2,8)$ \\
\hline C. glabrata & 105 & 4 & 8 & $<0,25->32$ & $84(80)$ & $15(14,3)$ & $6(5,7)$ \\
\hline C. parapsilosis & 46 & 0,5 & 2 & $<0,25-32$ & $44(95,6)$ & $2(4,4)$ & - \\
\hline C. tropicalis & 42 & 0,5 & 2 & $0,25->32$ & $39(92,8)$ & $1(2,4)$ & $2(4,8)$ \\
\hline C. kefyr & 11 & 0,5 & 0,5 & $0,25-0,5$ & $11(100)$ & - & - \\
\hline C. krusei & 8 & 16 & $>32$ & $8->32$ & $1(12,5)$ & $5(62,5)$ & $2(25)$ \\
\hline
\end{tabular}




\subsubsection{Itraconazol}

\subsubsection{C. albicans}

87,9\% (322 Isolate) der C. albicans-Isolate waren empfindlich gegenüber Itraconazol. 5,9\% (19 Isolate) waren dosisabhängig empfindlich und 6,2\% (20 Isolate) waren resistent. Die Hemmkonzentrationen lagen im Bereich der Konzentrationsstufen von $0,0313 \mu \mathrm{g} / \mathrm{ml}$ bis $16 \mu \mathrm{g} / \mathrm{ml}$. $\mathrm{MHK}_{50}$ und $\mathrm{MHK}_{90}$ betrugen $0,0625 \mu \mathrm{g} / \mathrm{ml}$ sowie $0,25 \mu \mathrm{g} / \mathrm{ml}$ (Tabelle $4-6$ ).

\subsubsection{C. glabrata}

$3,8 \%$ (vier Stämme) von C. glabrata waren empfindlich und 37,1\% (39 Isolate) waren dosisabhängig empfindlich. Die Resistenzrate lag mit 62 resistenten Stämmen bei $59,1 \%$. Die MHKs lagen im Bereich von $<0,0313 \mu \mathrm{g} / \mathrm{ml}$ bis $16 \mu \mathrm{g} / \mathrm{ml}$. Die $\mathrm{MHK}_{50}$ und $\mathrm{MHK}_{90}$ wurden mit $1 \mu \mathrm{g} / \mathrm{ml}$ und $2 \mu \mathrm{g} / \mathrm{ml}$ berechnet (Tabelle 4-6).

\subsubsection{C. parapsilosis}

73,9\% (34 Stämme) der C. parapsilosis-Isolate waren Itraconazol-empfindlich. 23,9\% (elf Isolate) wiesen eine dosisabhängige Empfindlichkeit auf, 2,2\% (ein Stamm) waren resistent. Die MHKs lagen innerhalb des Bereichs von $0,0625 \mu \mathrm{g} / \mathrm{ml}$ bis $1 \mu \mathrm{g} / \mathrm{ml}$. Die $\mathrm{MHK}_{50}$ betrug $0,125 \mu \mathrm{g} / \mathrm{ml}$ und die $\mathrm{MHK}_{90} 0,25 \mu \mathrm{g} / \mathrm{ml}$ (Tabelle 4-6).

\subsubsection{C. tropicalis}

Mit 21 Stämmen waren $50 \%$ der C. tropicalis-Isolate empfindlich gegenüber Itraconazol. 18 Isolate $(42,9 \%)$ waren dosisabhängig empfindlich. Vollständig resistent waren drei Isolate $(7,1 \%)$. Die Hemmkonzentrationen reichten von $0,0313 \mu \mathrm{g} / \mathrm{ml}$ bis $16 \mu \mathrm{g} / \mathrm{ml}$. MHK ${ }_{50}$ und $\mathrm{MHK}_{90}$ betrugen $0,125 \mu \mathrm{g} / \mathrm{ml}$ und $0,5 \mu \mathrm{g} / \mathrm{ml}$ (Tabelle 4-6).

\subsubsection{C. kefyr}

Drei der C. kefyr-Isolate $(27,3 \%)$ waren empfindlich gegenüber Itraconazol. Demgegenüber standen acht Isolate $(72,7 \%)$, die dosisabhängig empfindlich waren. Vollständige Resistenzen gegenüber Itraconazol traten nicht auf. Die MHKs lagen zwischen $0,0625 \mu \mathrm{g} / \mathrm{ml}$ und $0,25 \mu \mathrm{g} / \mathrm{ml}$. Die $\mathrm{MHK}_{50} \mathrm{bzw}$. MHK 90 betrugen jeweils $0,25 \mu \mathrm{g} / \mathrm{ml}$ (Tabelle 4-6). 


\subsubsection{C. krusei}

Keines der C. krusei-Isolate war sensibel. Drei Stämme $(37,5 \%)$ waren dosisabhängig sensibel. Mit fünf Stämmen (62,5\%) war der größte Teil Itraconazolresistent. Die minimalen Hemmkonzentrationen lagen innerhalb des Bereichs von $0,25 \mu \mathrm{g} / \mathrm{ml}$ bis $1 \mu \mathrm{g} / \mathrm{ml}$. $\mathrm{MHK}_{50}$ und $\mathrm{MHK}_{90}$ betrugen jeweils $1 \mu \mathrm{g} / \mathrm{ml}$ (Tabelle 4-6).

\subsubsection{Andere Spezies}

Einer der Cr. neoformans-Stämme war empfindlich, die anderen Isolate waren dosisabhängig empfindlich. Unter den $S$. cerevisiae-Isolaten befand sich ein dosisabhängig empfindlicher Stamm. Die anderen vier Isolate waren resistent gegenüber Itraconazol. Drei der C. guilliermondii-Isolate waren dosisabhängig empfindlich; das vierte Isolat wies eine Itraconazol-Resistenz auf. Die $C$. dubliniensis-Isolate waren empfindlich; ebenso die Isolate der Spezies $C$. catenulata, C. colliculosa C. intermedia und Trichosporon. Unter den C. inconspicua-Isolaten befanden sich zwei dosisabhängig empfindliche Isolate sowie ein resistentes Isolat. Ein C. Iusitaniae-Isolat war empfindlich, das andere dosisabhängig empfindlich. Die Isolate der Spezies $C$. norvegensis und $C$. famata waren dosisabhängig empfindlich. Die Stämme der Spezies $C$. rugosa, $C$. utilis und G. capitatum wiesen eine Itraconazol-Resistenz auf. Die MHKs lagen im Bereich zwischen 0,0313 $\mu \mathrm{g} / \mathrm{ml}$ bis $16 \mu \mathrm{g} / \mathrm{ml}$ (Tabelle 7-3, S. 98).

Die genaue Verteilung der Isolate über die unterschiedlichen Konzentrationsstufen ist Tabelle 7-3 (S. 98) zu entnehmen.

Tabelle 4-6: $\mathrm{MHK}_{50}, \mathrm{MHK}_{90}$ und die Bereiche der minimalen Hemmkonzentrationen (in $\mu \mathrm{g} / \mathrm{ml}$ ) sowie die Empfindlichkeit der häufigsten Spezies gegenüber Itraconazol

\begin{tabular}{|l|c|c|c|c|c|c|c|}
\hline & & & & \multirow{2}{*}{\begin{tabular}{c} 
Bereich \\
\cline { 5 - 8 }
\end{tabular}} & & & \multicolumn{3}{|c|}{ N (\%) der Isolate } \\
\cline { 5 - 9 } Itraconazol & $\mathbf{N}$ & MHK $_{\mathbf{5 0}}$ & MHK $_{\mathbf{9 0}}$ & der MHKs & sensibel & d-sensibel & resistent \\
\hline Gesamt & 567 & 0,1 & 1 & $<0,0313-16$ & $357(63)$ & $109(19,2)$ & $101(17,8)$ \\
\hline C. albicans & 322 & 0,1 & 0,25 & $0,0313-16$ & $283(87,9)$ & $19(5,9)$ & $20(6,2)$ \\
\hline C. glabrata & 105 & 1 & 2 & $<0,0313-16$ & $4(3,8)$ & $39(37,1)$ & $62(59,1)$ \\
\hline C. parapsilosis & 46 & 0,1 & 0,25 & $0,0625-1$ & $34(73,9)$ & $11(23,9)$ & $1(2,2)$ \\
\hline C. tropicalis & 42 & 0,1 & 0,5 & $0,0313-16$ & $21(50)$ & $18(42,9)$ & $3(7,1)$ \\
\hline C. kefyr & 11 & 0,3 & 0,25 & $0,0625-0,25$ & $3(27,3)$ & $8(72,7)$ & - \\
\hline C. krusei & 8 & 1 & 1 & $0,25-1$ & - & $3(37,5)$ & $5(62,5)$ \\
\hline
\end{tabular}




\subsubsection{Voriconazol}

\subsubsection{C. albicans}

Mit 96,9\% (312 Stämme) waren die C. albicans-Isolate überwiegend empfindlich gegenüber Voriconazol. 0,3\% (ein Isolat) waren dosisabhängig empfindlich, die restlichen neun Isolate $(2,8 \%)$ waren resistent. Die MHKs lagen im Bereich der Konzentrationsstufen von $<0,0313$ bis $16 \mu \mathrm{g} / \mathrm{ml}$. Die $\mathrm{MHK}_{50}$ betrug $<0,0313 \mu \mathrm{g} / \mathrm{ml}$ und die $\mathrm{MHK}_{90} 0,0625 \mu \mathrm{g} / \mathrm{ml}$ (Tabelle 4-7).

\subsubsection{C. glabrata}

$87,6 \%$ (92 Stämme) von C. glabrata waren empfindlich. 8,6\% (neun Isolate) waren dosisabhängig empfindlich und $3,8 \%$ (vier Isolate) waren resistent. Die MHKs lagen innerhalb des Konzentrationsbereichs von $<0,0313 \mu \mathrm{g} / \mathrm{ml} \mathrm{bis} 4 \mu \mathrm{g} / \mathrm{ml}$. Die $\mathrm{MHK}_{50}$ und $\mathrm{MHK}_{90}$ betrugen $0,125 \mu \mathrm{g} / \mathrm{ml}$ und $1 \mu \mathrm{g} / \mathrm{ml}$ (Tabelle 4-7).

\subsubsection{C. parapsilosis}

$97,8 \%$ (45 Stämme) der C. parapsilosis-Isolate waren sensibel. Ein Stamm $(2,2 \%)$ war resistent. Die MHKs lagen im Bereich zwischen $<0,0313 \mu \mathrm{g} / \mathrm{ml}$ und $16 \mu \mathrm{g} / \mathrm{ml}$. Die $\mathrm{MHK}_{50}$ lag bei $0,0625 \mu \mathrm{g} / \mathrm{ml}$ und die $\mathrm{MHK}_{90}$ bei $0,125 \mu \mathrm{g} / \mathrm{ml}$ (Tabelle 4-7).

\subsubsection{C. tropicalis}

$85,7 \%$ (36 Isolate) der C. tropicalis-Isolate waren empfindlich gegenüber Voriconazol. 14,3\% (sechs Isolate) wiesen eine Voriconazol-Resistenz auf. Die minimalen Hemmkonzentrationen lagen im Bereich zwischen <0,0313 und $16 \mu \mathrm{g} / \mathrm{ml}$. $\mathrm{MHK}_{50}$ und $\mathrm{MHK}_{90}$ betrugen $0,0313 \mu \mathrm{g} / \mathrm{ml}$ und $16 \mu \mathrm{g} / \mathrm{ml}$. (Tabelle 4-7).

\subsubsection{C. kefyr}

Die C. kefyr-Isolate waren durchgängig empfindlich gegenüber Voriconazol. Die MHKs lagen im Bereich von $<0,0313$ bis $0,0625 \mu \mathrm{g} / \mathrm{ml}$. Die $\mathrm{MHK}_{50}$ und $\mathrm{MHK}_{90}$ wurden bei $0,0313 \mu \mathrm{g} / \mathrm{ml}$ und $0,0625 \mu \mathrm{g} / \mathrm{ml}$ ermittelt (Tabelle $4-7$ ).

\subsubsection{C. krusei}

Sechs $(75 \%)$ der C. krusei-Isolate waren empfindlich. Zwei Isolate (25\%) wiesen eine dosisabhängige Empfindlichkeit auf. Die MHKs lagen im Konzentrationsbereich von $0,0625 \mu \mathrm{g} / \mathrm{ml}$ bis $1 \mu \mathrm{g} / \mathrm{ml}$. $\mathrm{MHK}_{50}$ und $\mathrm{MHK}_{90}$ betrugen $0,25 \mu \mathrm{g} / \mathrm{ml}$ sowie $1 \mu \mathrm{g} / \mathrm{ml}$ (Tabelle $4-7$ ). 


\subsubsection{Andere Spezies}

Drei der C. guilliermondii-Isolate waren empfindlich, der vierte Stamm dosisabhängig empfindlich. Eines der beiden C. norvegensis-Isolate war empfindlich, das andere dosisabhängig empfindlich. Der C. rugosa-Stamm war resistent. Die Isolate der weiteren Spezies, so auch $\mathrm{Cr}$. neoformans, waren durchgängig empfindlich (Tabelle 7-4, S. 99).

Die genaue Verteilung der Isolate über die unterschiedlichen Konzentrationsstufen ist Tabelle 7-4 (S. 99) zu entnehmen.

Tabelle 4-7: $\mathrm{MHK}_{50}, \mathrm{MHK}_{90}$ und die Bereiche der minimalen Hemmkonzentrationen (in $\mu \mathrm{g} / \mathrm{ml}$ ) sowie die Empfindlichkeit der häufigsten Spezies gegenüber Voriconazol

\begin{tabular}{|c|c|c|c|c|c|c|c|}
\hline \multirow[b]{2}{*}{ Voriconazol } & \multirow[b]{2}{*}{$\mathbf{N}$} & \multirow[b]{2}{*}{$\mathrm{MHK}_{50}$} & \multirow[b]{2}{*}{$\mathrm{MHK}_{90}$} & \multirow{2}{*}{$\begin{array}{c}\text { Bereich } \\
\text { der MHKs }\end{array}$} & \multicolumn{3}{|c|}{$\mathbf{N}(\%)$ der Isolate } \\
\hline & & & & & sensibel & d-sensibel & resistent \\
\hline Gesamt & 567 & 0 & 0,25 & $<0,0313-16$ & $535(94,4)$ & $13(2,3)$ & $19(3,3)$ \\
\hline C. albicans & 322 & $<0,0313$ & 0,0625 & $<0,0313-16$ & $312(96,9)$ & $1(0,3)$ & $9(2,8)$ \\
\hline C. glabrata & 105 & 0,1 & 1 & $<0,0313-4$ & $92(87,6)$ & $9(8,6)$ & $4(3,8)$ \\
\hline C. parapsilosis & 46 & 0,1 & 0,125 & $<0,0313-16$ & $45(97,8)$ & - & $1(2,2)$ \\
\hline C. tropicalis & 42 & 0 & 16 & $<0,0313-16$ & $36(85,7)$ & - & $6(14,3)$ \\
\hline C. kefyr & 11 & 0 & 0,0625 & $<0,0313-0,0625$ & $11(100)$ & - & - \\
\hline C. krusei & 8 & 0,3 & 1 & $0,0625-1$ & $6(75)$ & $2(25)$ & - \\
\hline
\end{tabular}

\subsubsection{Kreuzresistenzen der Azole}

Zwischen den Azolen Fluconazol, Itraconazol und Voriconazol lagen Kreuzresistenzen (dosisabhängig empfindlich oder resistent) vor. Davon betroffen waren insgesamt 53 Isolate.

\section{Fluconazol und Itraconazol}

34 Isolate, bei denen Fluconazol nicht effektiv wirkte, wiesen auch herabgesetzte Empfindlichkeit gegenüber Itraconazol auf, ohne jedoch gegenüber Voriconazol resistent zu sein. In sieben dieser Fälle handelte es sich um eine vollständige Resistenz; dazu zählten jeweils ein Isolat der Spezies C. albicans, C. glabrata, C. 
tropicalis, G. capitatum und S. cerevisiae sowie zwei Isolate von C. krusei. Auffällig war, dass sieben der insgesamt acht vorkommenden C. krusei-Isolate sowie alle drei $C$. inconspicua-Isolate eine herabgesetzte Empfindlichkeit gegenüber den zwei Azolen aufwiesen. Voriconazol, als Azol der jüngeren Generation, war jedoch gegenüber den gesamten 34 Isolaten effektiv wirksam.

\section{Itraconazol und Voriconazol}

Acht Isolate wiesen eine herabgesetzte Empfindlichkeit gegenüber Itraconazol und Voriconazol auf. Es handelte sich um vollständige Resistenzen gegenüber beiden Azolen. Ausnahme war ein C. tropicalis-Isolat, das gegenüber Itraconazol dosisabhängig empfindlich war. Die restlichen sieben Isolate setzten sich aus einem weiteren $C$. tropicalis-Stamm sowie fünf $C$. albicans-Isolaten und einem $C$. rugosa-Isolat zusammen. Es bestand jedoch bei allen acht Stämmen Empfindlichkeit gegenüber Fluconazol.

\section{Dreifachresistenzen}

In elf Fällen lag eine herabgesetzte Empfindlichkeit gegenüber allen drei Azolen vor. Dabei handelte es sich um $C$. albicans (drei dreifach vollständig resistente Isolate), C. glabrata (zwei dreifach vollständig resistente, zwei gegenüber Fluconazol und Itraconazol resistente jedoch Voriconazol gegenüber dosisabhängig empfindliche Isolate, ein Fluconazol und Voriconazol-resistentes Isolat mit dosisabhängiger Empfindlichkeit gegenüber Itraconazol sowie ein gegenüber Voriconazol vollständig resistentes Isolat, das gegenüber Fluconazol und Itraconazol eine dosisabhängige Empfindlichkeit aufwies) sowie $C$. guilliermondii (ein Isolat mit vollständiger Resistenz gegenüber Itraconazol und dosisabhängiger Empfindlichkeit gegenüber Fluconazol und Voriconazol) und $C$. tropicalis (ein dreifach vollständig resistentes Isolat).

Die Abbildung 4-4 zeigt die Anteile der Spezies an den Kreuzresistenzen zwischen den verschiedenen Azolen. 


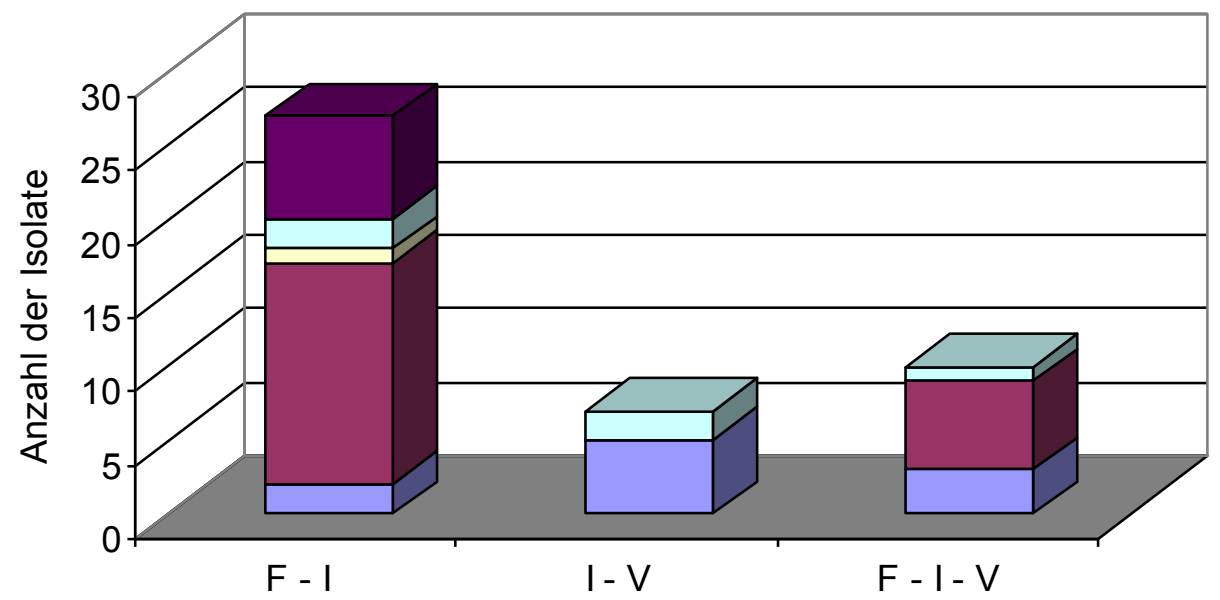

$\square$ C. albicans $\square$ C. glabrata $\square$ C. parapsilosis $\square$ C. tropicalis $\square$ C. krusei

Abbildung 4-4: Kreuzresistenzen zwischen Fluconazol und Itraconazol (F-I), Itraconazol und Voriconazol (I-V) sowie Dreifachresistenzen (F-I-V)

\subsubsection{Besonderheiten der Spezies C. glabrata gegenüber den getesteten Antimykotika}

Der Anteil von C. glabrata an den 53 Isolaten mit Kreuzresistenzen, war mit 21 Stämmen am größten (39,6\%). Daher wird diese Gruppe einer separaten Analyse unterzogen. Interessant war dabei, inwieweit die Isolate, die gegenüber Fluconazol durch eine herabgesetzte Empfindlichkeit (MHK $\geq 16 \mu \mathrm{g} / \mathrm{ml}$ ) auffielen, den anderen Antimykotika gegenüber empfindlich waren.

Gegenüber 5-Fluorcytosin waren alle Isolate empfindlich. Gegenüber Itraconazol war jedoch keines der Isolate empfindlich. Alle anderen Isolate wiesen eine herabgesetzte Empfindlichkeit auf (sieben waren dosisabhängig empfindlich und 14 resistent). Die Empfindlichkeit gegenüber Voriconazol war mit elf empfindlichen, sechs dosisabhängig empfindlichen und vier resistenten Isolaten besser. Die MHKs von Caspofungin lagen mit einem Maximum von 0,5 $\mu \mathrm{g} / \mathrm{ml}$ in einem niedrigen Bereich, so dass von Empfindlichkeit ausgegangen werden kann. Gegenüber Amphotericin B waren alle Isolate empfindlich.

Die genauen Ergebnisse der MHKs sind für die fünf Antimykotika in der Tabelle 4-8 aufgeführt. 
Tabelle 4-8: Anzahl der Isolate mit der jeweiligen MHK (in $\mu \mathrm{g} / \mathrm{ml}$ ) der fünf verschiedenen Antimykotika für die Isolate, die in der Gruppe der kreuzresistenten C. glabrata-Stämme durch herabgesetzte Empfindlichkeit gegenüber Fluconazol auffielen. Grau hinterlegt ist jeweils der dosisabhängig empfindliche/intermediäre Bereich der Verdünnungsstufen; bei Amphotericin B ist der empfindliche von dem resistenten Bereich der Verdünnungsstufen durch den schwarzen Balken getrennt

\begin{tabular}{|l|c|c|c|c|c|c|c|c|c|c|c|c|c|}
\hline & \multicolumn{8}{|c|}{ Anzahl der Isolate mit einer MHK von } \\
\cline { 2 - 16 } & $\mathbf{0} \mathbf{0 , 0 3 1 3}$ & $\mathbf{0 , 0 3 1 3}$ & $\mathbf{0 , 0 6 2 5}$ & $\mathbf{0 , 1 2 5}$ & $\mathbf{0 , 2 5}$ & $\mathbf{0 , 5}$ & $\mathbf{1}$ & $\mathbf{2}$ & $\mathbf{4}$ & $\mathbf{8}$ & $\mathbf{1 6}$ & $>\mathbf{1 6}$ \\
\hline Itraconazol & & & & & 1 & 6 & 5 & 3 & & 6 & \\
\hline Voriconazol & 1 & 1 & 1 & 2 & 4 & 2 & 4 & 2 & 4 & & & \\
\hline Caspofungin & 1 & 10 & 8 & 1 & & 1 & & & & & \\
\hline Amphotericin B & & & & & & 6 & 15 & & & & \\
\hline 5-Fluorcytosin & & & 20 & & 1 & & & & & & \\
\hline
\end{tabular}

\section{Zusammenfassung}

Die C. albicans-Isolate wiesen vor allem Kreuzresistenzen zwischen Itraconazol und Voriconazol auf. Sieben der insgesamt acht C. krusei-Isolate $(87,5 \%)$ wiesen eine Kreuzresistenz zwischen Fluconazol und Itraconazol auf. C. tropicalis zeigte bei allen Azolen gleichmäßig geringe Resistenzen. Eindeutige Trends waren jedoch nicht zu erkennen. Die Spezies C. glabrata kam mit einem Anteil von über einem Drittel $(39,6 \%)$ der Isolate, bei denen eine Kreuzresistenz vorlag, am häufigsten vor. Diese Isolate waren gleichzeitig gegenüber 5-Fluorcytosin und Amphotericin B empfindlich. Die MHKs bezüglich Caspofungin waren $\leq 0,5 \mu \mathrm{g} / \mathrm{ml}$. Gegenüber den anderen Azolen, insbesondere gegenüber Itraconazol, bestanden jedoch zahlreiche Resistenzen.

\subsubsection{Caspofungin}

\subsubsection{C. albicans}

Alle 322 Isolate waren empfindlich $(\leq 0,25 \mu \mathrm{g} / \mathrm{ml})$ gegenüber Caspofungin. Die MHKs lagen im Bereich der Konzentrationsstufen von $<0,0313 \mu \mathrm{g} / \mathrm{ml}$ bis $0,25 \mu \mathrm{g} / \mathrm{ml}$. Die $\mathrm{MHK}_{50}$ wurde mit $0,0313 \mu \mathrm{g} / \mathrm{ml}$ und die $\mathrm{MHK}_{90}$ mit $0,0625 \mu \mathrm{g} / \mathrm{ml}$ ermittelt (Tabelle 4-9). 


\subsubsection{C. glabrata}

99 Isolate $(94,3 \%)$ waren empfindlich $(\leq 0,125 \mu \mathrm{g} / \mathrm{ml})$, fünf Isolate $(4,8 \%)$ waren intermediär empfindlich $(0,25 \mu \mathrm{g} / \mathrm{ml})$ und ein Isolat $(0,9 \%)$ war resistent $(\geq 0,5 \mu \mathrm{g} / \mathrm{ml})$. Die MHKs lagen im Konzentrationsbereich von $<0,0313 \mu \mathrm{g} / \mathrm{ml}$ bis $0,5 \mu \mathrm{g} / \mathrm{ml}$. Die $\mathrm{MHK}_{50}$ betrug $0,0313 \mu \mathrm{g} / \mathrm{ml}$ und die $\mathrm{MHK}_{90} 0,0625 \mu \mathrm{g} / \mathrm{ml}$ (Tabelle 4-9).

\subsubsection{C. parapsilosis}

Die 46 Isolate waren sensibel $(\leq 2 \mu \mathrm{g} / \mathrm{ml})$. Die MHKs lagen im Bereich der Konzentrationsstufen zwischen $0,0313 \mu \mathrm{g} / \mathrm{ml}$ und $0,5 \mu \mathrm{g} / \mathrm{ml}$. Die $\mathrm{MHK}_{50}$ betrug $0,25 \mu \mathrm{g} / \mathrm{ml}$ und die $\mathrm{MHK}_{90} 0,5 \mu \mathrm{g} / \mathrm{ml}$ (Tabelle 4-9).

\subsubsection{C. tropicalis}

Auch alle 42 C. tropicalis-Stämme waren empfindlich $(\leq 0,25 \mu \mathrm{g} / \mathrm{ml})$. Die MHKs $\operatorname{der}$ C. tropicalis-Isolate lagen im Konzentrationsbereich zwischen $<0,0313 \mu \mathrm{g} / \mathrm{ml}$ und $0,25 \mu \mathrm{g} / \mathrm{ml}$. Die $\mathrm{MHK}_{50}$ betrug $0,0313 \mu \mathrm{g} / \mathrm{ml}$, die $\mathrm{MHK}_{90} 0,125 \mu \mathrm{g} / \mathrm{ml}$ (Tabelle 49).

\subsubsection{C. kefyr}

Es lag durchgängige Empfindichkeit $(\leq 2 \mu \mathrm{g} / \mathrm{ml})$ der 11 Isolate vor. Die MHKs der C. kefyr-Isolate lagen im Bereich der Konzentrationsstufen von $<0,0313 \mu \mathrm{g} / \mathrm{ml}$ bis $0,0625 \mu \mathrm{g} / \mathrm{ml}$. Die $\mathrm{MHK}_{50}$ sowie die $\mathrm{MHK}_{90}$ betrugen jeweils $0,0625 \mu \mathrm{g} / \mathrm{ml}$ (Tabelle 4-9).

\subsubsection{C. krusei}

Auch die C. krusei-Stämme waren durchgängig empfindlich $(\leq 0,25 \mu \mathrm{g} / \mathrm{ml})$. Die MHKs der C. krusei-Isolate lagen im Konzentrationsbereich zwischen $0,0625 \mu \mathrm{g} / \mathrm{ml}$ und $0,25 \mu \mathrm{g} / \mathrm{ml}$. $\mathrm{MHK}_{50}$ und $\mathrm{MHK}_{90}$ betrugen $0,125 \mu \mathrm{g} / \mathrm{ml}$ und $0,25 \mu \mathrm{g} / \mathrm{ml}$ (Tabelle 4-9).

\subsubsection{Andere Spezies}

Gegenüber den fünf $\mathrm{Cr}$. neoformans-Stämmen war Caspofungin nicht wirksam (100\% Resistenz bei MHKs von 8 bis $16 \mu \mathrm{g} / \mathrm{ml})$. Dies galt auch für die $C$. guilliermondii-Isolate (MHKs von $16 \mu \mathrm{g} / \mathrm{ml}$ ), das Geotrichum-Isolat (MHK von $8 \mu \mathrm{g} / \mathrm{ml}$ sowie die zwei Trichosporon-Stämme (MHK von 8 und $16 \mu \mathrm{g} / \mathrm{m}$ ). Die weiteren Spezies wiesen MHKs $\leq 1 \mu \mathrm{g} / \mathrm{ml}$ auf und waren somit sensibel (Tabelle 
7-5, S. 100).

Die genaue Verteilung der Isolate über die unterschiedlichen Konzentrationsstufen ist Tabelle 7-5 (S. 100) zu entnehmen.

Tabelle 4-9: $\mathrm{MHK}_{50}, \mathrm{MHK}_{90}$ und die Bereiche der minimalen Hemmkonzentrationen (in $\mu \mathrm{g} / \mathrm{ml}$ ) sowie die Empfindlichkeit der häufigsten Spezies gegenüber Caspofungin

\begin{tabular}{|c|c|c|c|c|c|c|c|}
\hline \multirow[b]{2}{*}{ Caspofungin } & \multirow[b]{2}{*}{$\mathbf{N}$} & \multirow[b]{2}{*}{$\mathrm{MHK}_{50}$} & \multirow[b]{2}{*}{ MHK $_{90}$} & \multirow{2}{*}{$\begin{array}{c}\text { Bereich } \\
\text { der MHKs }\end{array}$} & \multicolumn{3}{|c|}{ N (\%) der Isolate } \\
\hline & & & & & sensibel & intermediär & resistent \\
\hline Gesamt & 567 & 0 & 0,25 & $<0,0313-16$ & $549(96,8)$ & $5(1)$ & $13(2,3)$ \\
\hline C. albicans & 322 & 0 & 0,0625 & $<0,0313-0,25$ & $322(100)$ & - & - \\
\hline C. glabrata & 105 & 0 & 0,0625 & $<0,0313-0,5$ & $99(94,3)$ & $5(4,8)$ & $1(0,9)$ \\
\hline C. parapsilosis & 46 & 0,3 & 0,5 & $0,0313-0,5$ & $46(100)$ & - & - \\
\hline C. tropicalis & 42 & 0 & 0,125 & $<0,0313-0,25$ & $42(100)$ & - & - \\
\hline C. kefyr & 11 & 0,1 & 0,0625 & $<0,0313-0,0625$ & $11(100)$ & - & - \\
\hline C. krusei & 8 & 0,1 & 0,25 & $0,0625-0,25$ & $8(100)$ & - & - \\
\hline
\end{tabular}

\subsubsection{Amphotericin B}

\subsubsection{C. albicans}

Alle C. albicans-Isolate waren Amphotericin-B-empfindlich. Die MHKs lagen im Bereich der Konzentrationsstufen zwischen $0,0625 \mu \mathrm{g} / \mathrm{ml}$ und $1 \mu \mathrm{g} / \mathrm{ml}$. Die $\mathrm{MHK}_{50}$ und $\mathrm{MHK}_{90}$ betrugen $0,5 \mu \mathrm{g} / \mathrm{ml}$ und $1 \mu \mathrm{g} / \mathrm{ml}$ (Tabelle $\left.4-10\right)$.

\subsubsection{C. glabrata}

Die C. glabrata-Isolate waren durchgängig empfindlich. Die MHKs lagen innerhalb der Konzentrationsbereiche von $0,25 \mu \mathrm{g} / \mathrm{ml}$ bis $1 \mu \mathrm{g} / \mathrm{ml}$. Die $\mathrm{MHK}_{50}$ und MHK $K_{90}$ betrugen jeweils $1 \mu \mathrm{g} / \mathrm{ml}$ (Tabelle $\left.4-10\right)$.

\subsubsection{C. parapsilosis}

Auch alle C. parapsilosis-Isolate waren Amphotericin-B-empfindlich. Die MHKs lagen im Konzentrationsbereich von $0,25 \mu \mathrm{g} / \mathrm{ml}$ bis $1 \mu \mathrm{g} / \mathrm{ml}$. Die $\mathrm{MHK}_{50}$ bzw. $\mathrm{MHK}_{90}$ wurden jeweils bei $1 \mu \mathrm{g} / \mathrm{ml}$ ermittelt (Tabelle 4-10). 


\subsubsection{C. tropicalis}

Die MHKs der ebenfalls empfindlichen C. tropicalis-Isolate lagen im Bereich zwischen 0,25 und $1 \mu \mathrm{g} / \mathrm{ml}$. Die $\mathrm{MHK}_{50}$ und $\mathrm{MHK}_{90}$ lagen bei $1 \mu \mathrm{g} / \mathrm{ml}$ (Tabelle 410).

\subsubsection{C. kefyr}

Alle C. kefyr-Isolate waren empfindlich gegenüber Amphotericin B. Die MHKs lagen im Bereich der Konzentrationsstufen von $0,5 \mu \mathrm{g} / \mathrm{ml}$ und $1 \mu \mathrm{g} / \mathrm{ml}$. Sowohl $\mathrm{MHK}_{50}$ als auch $\mathrm{MHK}_{90}$ betrugen $1 \mu \mathrm{g} / \mathrm{ml}$ (Tabelle 4-10).

\subsubsection{C. krusei}

Die C. krusei-Isolate waren ebenso durchgängig empfindlich. Die MHKs lagen innerhalb des Bereichs von $0,5 \mu \mathrm{g} / \mathrm{ml}$ bis $1 \mu \mathrm{g} / \mathrm{ml}$. Die $\mathrm{MHK}_{50}$ und $\mathrm{MHK}_{90}$ betrugen in beiden Fällen $1 \mu \mathrm{g} / \mathrm{ml}$ (Tabelle 4-10).

\subsubsection{Andere Spezies}

Auch alle Isolate der anderen Spezies waren Amphotericin-B-empfindlich Die MHKs lagen innerhalb des Bereichs von $0,0625 \mu \mathrm{g} / \mathrm{ml}$ bis $1 \mu \mathrm{g} / \mathrm{ml}$ (Tabelle $7-6$, S. 101).

Die genaue Verteilung der Isolate über die unterschiedlichen Konzentrationsstufen ist Tabelle 7-6 (S. 101) zu entnehmen.

Tabelle 4-10: $\mathrm{MHK}_{50}, \mathrm{MHK}_{90}$ und die Bereiche der minimalen Hemmkonzentrationen (in $\mu \mathrm{g} / \mathrm{ml}$ ) sowie die Empfindlichkeit der häufigsten Spezies gegenüber Amphotericin B

\begin{tabular}{|c|c|c|c|c|c|c|}
\hline \multirow[b]{2}{*}{ Amphotericin B } & \multirow[b]{2}{*}{$\mathbf{N}$} & \multirow[b]{2}{*}{$\mathrm{MHK}_{50}$} & \multirow[b]{2}{*}{$\mathrm{MHK}_{90}$} & \multirow{2}{*}{$\begin{array}{c}\text { Bereich } \\
\text { der MHKs }\end{array}$} & \multicolumn{2}{|c|}{$\mathbf{N}(\%)$ der Isolate } \\
\hline & & & & & sensibel & resistent \\
\hline Gesamt & 567 & 0,5 & 1 & $0,0625-1$ & $567(100)$ & - \\
\hline C. albicans & 322 & 0,5 & 1 & $0,0625-1$ & $322(100)$ & - \\
\hline C. glabrata & 105 & 1 & 1 & $0,25-1$ & $105(100)$ & - \\
\hline C. parapsilosis & 46 & 1 & 1 & $0,25-1$ & $46(100)$ & - \\
\hline C. tropicalis & 42 & 1 & 1 & $0,25-1$ & $42(100)$ & - \\
\hline C. kefyr & 11 & 1 & 1 & $0,5-1$ & $11(100)$ & - \\
\hline C. krusei & 8 & 1 & 1 & $0,5-1$ & $8(100)$ & - \\
\hline
\end{tabular}




\subsubsection{5-Fluorcytosin}

\subsubsection{C. albicans}

Die 322 C. albicans-Isolate waren durchgängig empfindlich gegenüber 5 -Fluorcytosin. Die minimalen Hemmkonzentrationen lagen im Bereich von $<0,125 \mu \mathrm{g} / \mathrm{ml}$ bis $4 \mu \mathrm{g} / \mathrm{ml}$. Die $\mathrm{MHK}_{50}$ wurde mit $<0,125 \mu \mathrm{g} / \mathrm{ml}$, die $\mathrm{MHK}_{90}$ mit $0,125 \mu \mathrm{g} / \mathrm{ml}$ ermittelt (Tabelle 4-11).

\subsubsection{C. glabrata}

Auch alle 105 C. glabrata-Isolate waren empfindlich gegenüber 5-Fluorcytosin. Die Hemmkonzentrationen lagen im Bereich zwischen $<0,125 \mu \mathrm{g} / \mathrm{ml}$ bis $2 \mu \mathrm{g} / \mathrm{ml}$. $\mathrm{MHK}_{50}$ und die $\mathrm{MHK}_{90}$ betrugen jeweils $<0,125 \mu \mathrm{g} / \mathrm{ml}$ (Tabelle 4-11).

\subsubsection{C. parapsilosis}

Die C. parapsilosis-Isolate (46 Stämme) waren ebenfalls empfindlich gegenüber 5-Fluorcytosin. Die minimalen Hemmkonzentrationen lagen im Bereich von $<0,125 \mu \mathrm{g} / \mathrm{ml}$ bis $2 \mu \mathrm{g} / \mathrm{ml}$. Die $\mathrm{MHK}_{50}$ wurde mit $<0,125 \mu \mathrm{g} / \mathrm{ml}$ und die $\mathrm{MHK}_{90}$ mit $0,25 \mu \mathrm{g} / \mathrm{ml}$ berechnet (Tabelle $4-11$ ).

\subsubsection{C. tropicalis}

Die Empfindlichkeit von C. tropicalis (42 Isolate) unterschied sich deutlich von den bisher beschriebenen Spezies. Nur 16 Isolate $(38,1 \%)$ waren empfindlich und ein Isolat war intermediär empfindlich $(2,4 \%)$ gegenüber 5-Fluorcytosin. Eine Resistenz wiesen 25 Isolate (59,5\%) auf. Die minimalen Hemmkonzentrationen lagen innerhalb des Bereichs von $<0,125 \mu \mathrm{g} / \mathrm{ml}$ und $>16 \mu \mathrm{g} / \mathrm{ml}$. Die $\mathrm{MHK}_{50}$ und $\mathrm{MHK}_{90}$ wurden jeweils bei $>16 \mu \mathrm{g} / \mathrm{ml}$ ermittelt (Tabelle 4-11).

\subsubsection{C. kefyr}

Die elf C. kefyr-Isolate waren 5-Fluorcytosin-empfindlich. Die minimalen Hemmkonzentrationen lagen dabei im Bereich von $<0,125 \mu \mathrm{g} / \mathrm{ml}$ bis $4 \mu \mathrm{g} / \mathrm{ml}$. Die $\mathrm{MHK}_{50}$ bzw. MHK $\mathrm{M}_{90}$ betrugen jeweils $4 \mu \mathrm{g} / \mathrm{ml}$ (Tabelle 4-11).

\subsubsection{C. krusei}

Nur eins der acht $C$. krusei-Isolate war empfindlich gegenüber 5-Fluorcytosin. Die weiteren sieben Isolate wurden als intermediär empfindlich klassifiziert $(87,5 \%)$. Die minimalen Hemmkonzentrationen lagen im Bereich von $4 \mu \mathrm{g} / \mathrm{ml}$ bis $16 \mu \mathrm{g} / \mathrm{ml}$. Die $\mathrm{MHK}_{50}$ wurde bei $8 \mu \mathrm{g} / \mathrm{ml}$ und die $\mathrm{MHK}_{90}$ bei $16 \mu \mathrm{g} / \mathrm{ml}$ berechnet (Tabelle 4- 
11).

\subsubsection{Andere Spezies}

Zwei C. inconspicua-Isolate waren empfindlich, das dritte Isolat wies eine intermediäre Empfindlichkeit auf. Ein Trichosporon-Stamm war empfindlich, das andere Isolat war resistent. Das C. utilis-Isolat war intermediär empfindlich. Die Isolate der weiteren Spezies waren durchgängig 5-Fluorcytosin-empfindlich. Die MHKs lagen in dem Bereich von $<0,125 \mu \mathrm{g} / \mathrm{ml}$ bis $>16 \mu \mathrm{g} / \mathrm{ml}$ (Tabelle 7-7, S. 102).

Die genaue Verteilung der Isolate über die unterschiedlichen Konzentrationsstufen ist Tabelle 7-7 (S. 102) zu entnehmen.

Tabelle 4-11: $\mathrm{MHK}_{50}, \mathrm{MHK}_{90}$ und die Bereiche der minimalen Hemmkonzentrationen (in $\mu \mathrm{g} / \mathrm{ml}$ ) sowie die Empfindlichkeit der häufigsten Spezies gegenüber 5-Fluorcytosin

\begin{tabular}{|c|c|c|c|c|c|c|c|}
\hline \multirow[b]{2}{*}{ 5-Fluorcytosin } & \multirow[b]{2}{*}{$\mathbf{N}$} & \multirow[b]{2}{*}{$\mathrm{MHK}_{50}$} & \multirow[b]{2}{*}{$\mathrm{MHK}_{90}$} & \multirow{2}{*}{$\begin{array}{c}\text { Bereich } \\
\text { der MHKs }\end{array}$} & \multicolumn{3}{|c|}{$\mathbf{N}(\%)$ der Isolate } \\
\hline & & & & & sensibel & intermed. & resistent \\
\hline Gesamt & 567 & $<0,125$ & 1 & $<0,125->16$ & $531(93,6)$ & $10(1,8)$ & $26(4,6)$ \\
\hline C. albicans & 322 & $<0,125$ & 0,125 & $<0,125-4$ & $322(100)$ & - & - \\
\hline C. glabrata & 105 & $<0,125$ & $<0,125$ & $<0,125-2$ & $105(100)$ & - & - \\
\hline C. parapsilosis & 46 & $<0,125$ & 0,25 & $<0,125-2$ & $46(100)$ & - & - \\
\hline C. tropicalis & 42 & $>16$ & $>16$ & $<0,125->16$ & $16(38,1)$ & $1(2,4)$ & $25(59,5)$ \\
\hline C. kefyr & 11 & 4 & 4 & $<0,125-4$ & $11(100)$ & - & - \\
\hline C. krusei & 8 & 8 & 16 & $4-16$ & $1(12,5)$ & $7(87,5)$ & - \\
\hline
\end{tabular}

\subsubsection{Zusammenfassung aller Mikrodilutions-Ergebnisse}

Alle 567 Isolate wurden der Mikrodilutionsmethode gegenüber den sechs Antimykotika unterzogen. Der Anteil gegenüber Fluconazol empfindlicher Isolate lag bei 90,8\%. Die Isolate, die dosisabhängig oder resistent waren, gehörten vor allem zu den Spezies C. glabrata und C. krusei. Die Resistenzrate von Itraconazol lag mit 17,8\% deutlich über der der anderen Antimykotika. Den größten Anteil machten dabei die Spezies C. glabrata und C. krusei aus. Neben diesen Spezies war auch der Anteil der C. tropicalis-Isolate mit herabgesetzter 
Empfindlichkeit bemerkenswert. Gegenüber Voriconazol waren $94,4 \%$ der Isolate empfindlich. Erwähnenswert war dabei der hohe Anteil von resistenten $C$. tropicalis-Isolaten (14,3\%). Der größte Teil der Isolate war empfindlich gegenüber Caspofungin (96,8\%), wobei der Anteil von Isolaten mit herabgesetzter Sensibilität bei C. glabrata am höchsten war (4,8\% intermediäre und $0,9 \%$ resistente Isolate). Gegenüber Amphotericin B waren alle Isolate empfindlich. Es zeigte sich für 5-Fluorcytosin ein Anteil von 93,7\% empfindlicher Isolate. Für die Resistenzrate von 4,6\% waren vor allem die C. tropicalis-Stämme verantwortlich (25 C. tropicalis-Isolate von insgesamt 26 resistenten Isolaten). Eine Übersicht der Ergebnisse der sechs Antimykotika zeigt die Tabelle 4-12.

Tabelle 4-12: Vergleich der $\mathrm{MHK}_{50}$ und $\mathrm{MHK}_{90}$, den Bereichen der MHKs (in $\mu \mathrm{g} / \mathrm{ml}$ ) sowie der kategorialen Bewertung aller Isolate gegenüber den sechs verwendeten Antimykotika

\begin{tabular}{|l|c|c|c|c|c|c|c|}
\hline & \multirow{2}{*}{} & & & & & \multicolumn{3}{|c|}{ Anzahl (\%) der Isolate } \\
\cline { 6 - 9 } & & & & & & intermediärl & \\
Antimykotikum & $\mathbf{N}$ & MHK $_{\mathbf{5 0}}$ & MHK $_{\mathbf{9 0}}$ & Bereich der MHKs & sensibel & d-sensibel & resistent \\
\hline Fluconazol & 567 & 0,5 & 8 & $<0,25->32$ & $515(90,8)$ & $32(5,6)$ & $20(3,6)$ \\
\hline Itraconazol & 567 & 0,125 & 1 & $<0,0313-16$ & $357(63)$ & $109(19,2)$ & $101(17,8)$ \\
\hline Voriconazol & 567 & 0,0313 & 0,25 & $<0,0313-16$ & $535(94,4)$ & $13(2,3)$ & $19(3,3)$ \\
\hline Caspofungin & 567 & 0,0313 & 0,25 & $<0,0313-16$ & $549(96,8)$ & $5(1)$ & $13(2,3)$ \\
\hline Amphotericin B & 567 & 0,5 & 1 & $<0,0625-1$ & $567(100)$ & - & \\
\hline 5-Fluorcytosin & 567 & $<0,125$ & 1 & $<0,125->16$ & $531(93,6)$ & $10(1,8)$ & $26(4,6)$ \\
\hline
\end{tabular}

\subsection{Ergebnisse des Etests}

Wie im Abschnitt 4.2 werden auch die Ergebnisse des Etests für die einzelnen Antimykotika separat dargestellt. Mit dem Etest wurden alle 567 Isolate gegenüber Fluconazol und 5-Fluorcytosin getestet. Stämme, die hierbei eine herabgesetzte Empfindlichkeit aufwiesen, sowie alle C. glabrata-Isolate, wurden auch gegenüber den anderen vier Antimykotika getestet.

Die Bewertung der Etest-Ergebnisse basierte auf den vom Hersteller empfohlenen Breakpoints der NCCLS-Leitlinien (vgl. Abschnitt 3.5.4). 


\subsubsection{Fluconazol}

\subsubsection{C. albicans}

Alle 322 C. albicans-Isolate wurden mit dem Etest gegenüber Fluconazol getestet. Die MHKs der C. albicans-Isolate lagen im Konzentrationsbereich von $<0,25 \mu \mathrm{g} / \mathrm{ml}$ bis $>32 \mu \mathrm{g} / \mathrm{ml}$. Die $\mathrm{MHK}_{50}$ und $\mathrm{MHK}_{90}$ wurden mit $0,5 \mu \mathrm{g} / \mathrm{ml}$ und $1 \mu \mathrm{g} / \mathrm{ml}$ berechnet. 97,5\% (314 Stämme) waren empfindlich gegenüber Fluconazol. 0,6\% (zwei Isolate) waren dosisabhängig empfindlich und 1,9\% (sechs Isolate) waren resistent (Tabelle 4-13).

\subsubsection{C. glabrata}

Es wurden 105 C. glabrata-Stämme dem Etest unterzogen. Die MHKs lagen innerhalb des Bereichs von $0,25 \mu \mathrm{g} / \mathrm{ml}$ bis $>32 \mu \mathrm{g} / \mathrm{ml}$. Die $\mathrm{MHK}_{50}$ betrug $16 \mu \mathrm{g} / \mathrm{ml}$ während die $\mathrm{MHK}_{90}>32 \mu \mathrm{g} / \mathrm{ml}$ betrug. 34,3\% (36 Isolate) waren empfindlich. $51,4 \%$ (54 Stämme) waren nur dosisabhängig empfindlich gegenüber Fluconazol und bei 14,3\% (15 Stämme) lag eine Resistenz vor (Tabelle 4-13).

\subsubsection{C. parapsilosis}

Es wurden 46 C. parapsilosis-Isolate getestet. Die MHKs lagen im Konzentrationsbereich von $0,25 \mu \mathrm{g} / \mathrm{ml}$ bis $32 \mu \mathrm{g} / \mathrm{ml}$. Die $\mathrm{MHK}_{50}$ und $\mathrm{MHK}_{90}$ betrugen $0,5 \mu \mathrm{g} / \mathrm{ml}$ und $2 \mu \mathrm{g} / \mathrm{ml}$. 95,7\% (44 Stämme) waren empfindlich und 4,4\% (zwei Isolate) waren dosisabhängig empfindlich gegenüber Fluconazol (Tabelle 4-13).

\subsubsection{C. tropicalis}

42 C. tropicalis-Stämme wurden getestet. Die MHKs lagen im Bereich der Konzentrationsstufen zwischen $0,25 \mu \mathrm{g} / \mathrm{ml}$ und $>32 \mu \mathrm{g} / \mathrm{ml}$. $\mathrm{MHK}_{50}$ und $\mathrm{MHK}_{90}$ betrugen $1 \mu \mathrm{g} / \mathrm{ml}$ respektive $2 \mu \mathrm{g} / \mathrm{ml}$. 97,6\% (41 Isolate) waren empfindlich. Demgegenüber stand ein resistentes Isolat (2,4\%, Tabelle 4-13).

\subsubsection{C. kefyr}

Es wurden elf C. kefyr-Isolate getestet. Die MHKs lagen im Konzentrationsbereich zwischen $<0,25 \mu \mathrm{g} / \mathrm{ml}$ und $1 \mu \mathrm{g} / \mathrm{ml}$. Die $\mathrm{MHK}_{50} \mathrm{bzw}$. MHK $\mathrm{M}_{90}$ wurden bei $0,25 \mu \mathrm{g} / \mathrm{ml}$ und $1 \mu \mathrm{g} / \mathrm{ml}$ ermittelt. Alle elf Isolate waren empfindlich (Tabelle 4-13). 


\subsubsection{C. krusei}

Es wurden acht C. krusei-Isolate mit dem Etest getestet. Die MHKs erstreckten sich über den Konzentrationsbereich von $16 \mu \mathrm{g} / \mathrm{ml}$ bis $>32 \mu \mathrm{g} / \mathrm{ml}$. Die $\mathrm{MHK}_{50}$ und $\mathrm{MHK}_{90}$ betrugen $32 \mu \mathrm{g} / \mathrm{ml}$ und $>32 \mu \mathrm{g} / \mathrm{ml}$. Keines der Isolate war empfindlich. Jeweils die Hälfte (vier Isolate) war dosisabhängig empfindlich und resistent (Tabelle 4-13).

Die genaue Verteilung der Isolate über die unterschiedlichen Konzentrationsstufen ist Tabelle 7-2 (S. 97) zu entnehmen.

Tabelle 4-13: $\mathrm{MHK}_{50}$, $\mathrm{MHK}_{90}$ und die Bereiche der minimalen Hemmkonzentrationen (in $\mu \mathrm{g} / \mathrm{ml}$ ) sowie die Empfindlichkeit der häufigsten Spezies gegenüber Fluconazol

\begin{tabular}{|c|c|c|c|c|c|c|c|}
\hline \multirow[b]{2}{*}{ Fluconazol } & \multirow[b]{2}{*}{$\mathbf{N}$} & \multirow[b]{2}{*}{$\mathrm{MHK}_{50}$} & \multirow[b]{2}{*}{$\mathrm{MHK}_{90}$} & \multirow{2}{*}{$\begin{array}{c}\text { Bereich } \\
\text { der MHKs }\end{array}$} & \multicolumn{3}{|c|}{ N (\%) der Isolate } \\
\hline & & & & & sensibel & d-sensibel & resistent \\
\hline Gesamt & 567 & 0,5 & 16 & $<0,25->32$ & $466(82,2)$ & $69(12,2)$ & $32(5,6)$ \\
\hline C. albicans & 322 & 0,5 & 1 & $<0,25->32$ & $314(97,5)$ & $2(0,6)$ & $6(1,9)$ \\
\hline C. glabrata & 105 & 16 & $>32$ & $0,25->32$ & $36(34,3)$ & $54(51,4)$ & $15(14,3)$ \\
\hline C. parapsilosis & 46 & 0,5 & 2 & $0,25-32$ & $44(95,7)$ & $2(4,3)$ & - \\
\hline C. tropicalis & 42 & 1 & 2 & $0,25->32$ & $41(97,6)$ & - & $1(2,4)$ \\
\hline C. kefyr & 11 & 0,3 & 1 & $<0,25-1$ & $11(100)$ & - & - \\
\hline C. krusei & 8 & 32 & $>32$ & $16->32$ & - & $4(50)$ & $4(50)$ \\
\hline
\end{tabular}

\subsubsection{Itraconazol}

\subsubsection{C. albicans}

Es wurden 97 C. albicans-Isolate mit dem Etest getestet. Der Bereich der Konzentrationsstufen, in dem die MHKs lagen, erstreckte sich von $<0,0313 \mu \mathrm{g} / \mathrm{ml}$ bis $>16 \mu \mathrm{g} / \mathrm{ml}$. Die $\mathrm{MHK}_{50}$ betrug $0,0313 \mu \mathrm{g} / \mathrm{ml}$ und die $\mathrm{MHK}_{90} 0,125 \mu \mathrm{g} / \mathrm{ml}$. 92,8\% (90 Isolate) waren empfindlich. 2,1\% (zwei Isolate) waren dosisabhängig empfindlich. 5,1\% (fünf Stämme) wiesen eine Itraconazol-Resistenz auf (Tabelle 4-14). 


\subsubsection{C. glabrata}

Es wurden 104 C. glabrata-Isolate getestet. Die MHKs lagen innerhalb des Konzentrationsbereichs von $<0,0313 \mu \mathrm{g} / \mathrm{ml}$ bis $>16 \mu \mathrm{g} / \mathrm{ml}$. Die $\mathrm{MHK}_{50}$ und $\mathrm{MHK}_{90}$ betrugen $16 \mu \mathrm{g} / \mathrm{ml}$ und $>16 \mu \mathrm{g} / \mathrm{ml}$. Mit 94,2\% (98 Stämmen) wiesen die Isolate überwiegend eine Itraconazol-Resistenz auf. Nur 3,8\% (vier Stämme) waren empfindlich gegenüber Itraconazol und 1,9\% (zwei Isolate) waren dosisabhängig empfindlich (Tabelle 4-14).

\subsubsection{C. parapsilosis}

Alle 46 C. parapsilosis-Isolate wurden mit dem Etest getestet. Die MHKs lagen im Bereich der Konzentrationsstufen von $<0,0313 \mu \mathrm{g} / \mathrm{ml}$ bis $1 \mu \mathrm{g} / \mathrm{ml}$. Die $M \mathrm{~K}_{50}$ und $\mathrm{MHK}_{90}$ betrugen $0,0625 \mu \mathrm{g} / \mathrm{ml}$ und $0,125 \mu \mathrm{g} / \mathrm{ml}$. 91,3\% (42 Stämme) der Isolate waren empfindlich. Ein Isolat erwies sich als Itraconazol-resistent $(2,2 \%)$. Drei Isolate (6,5\%) waren dosisabhängig empfindlich (Tabelle 4-14).

\subsubsection{C. tropicalis}

42 C. tropicalis-Stämme wurden dem Etest unterzogen. Die MHKs lagen im Konzentrationsbereich zwischen $<0,0313 \mu \mathrm{g} / \mathrm{ml}$ und $>16 \mu \mathrm{g} / \mathrm{ml}$. Die $\mathrm{MHK}_{50}$ und $\mathrm{MHK}_{90}$ betrugen $0,0625 \mu \mathrm{g} / \mathrm{ml}$ und $1 \mu \mathrm{g} / \mathrm{ml}$. 64,3\% (27 Stämme) waren empfindlich, 21,4\% (neun Isolate) dosisabhängig empfindlich sowie 14,3\% (sechs Stämme) resistent (Tabelle 4-14).

\subsubsection{C. kefyr}

Es wurden sieben $C$. kefyr-Isolate getestet. Die MHKs lagen im Bereich von $<0,0313 \mu \mathrm{g} / \mathrm{ml}$ bis $0,0625 \mu \mathrm{g} / \mathrm{ml}$. Die $\mathrm{MHK}_{50}$ und $\mathrm{MHK}_{90}$ wurden jeweils bei $0,0625 \mu \mathrm{g} / \mathrm{ml}$ bestimmt. Die Isolate waren durchgängig Itraconazol-empfindlich (Tabelle 4-14).

\subsubsection{C. krusei}

Es wurden sieben C. krusei-Isolate getestet. Die MHKs lagen innerhalb des Bereichs der Konzentrationsstufen von $0,125 \mu \mathrm{g} / \mathrm{ml}$ bis $8 \mu \mathrm{g} / \mathrm{ml}$. Die $\mathrm{MHK}_{50}$ betrug $0,5 \mu \mathrm{g} / \mathrm{ml}$, die $\mathrm{MHK}_{90} 8 \mu \mathrm{g} / \mathrm{ml}$. 28,6\% (zwei Isolate) von C. krusei waren empfindlich. Mit einem Anteil von 42,8\% waren drei der Stämme dosisabhängig empfindlich. Zwei Isolate waren resistent (28,6\%, Tabelle 4-14).

Die genaue Verteilung der Isolate über die unterschiedlichen 
Konzentrationsstufen ist Tabelle 7-3 (S. 98) zu entnehmen.

Tabelle 4-14: $\mathrm{MHK}_{50}, \mathrm{MHK}_{90}$ und die Bereiche der minimalen Hemmkonzentrationen (in $\mu \mathrm{g} / \mathrm{ml}$ ) sowie die Empfindlichkeit der häufigsten Spezies gegenüber Itraconazol

\begin{tabular}{|l|c|c|c|c|c|c|c|}
\hline & & & & \multirow{2}{*}{\begin{tabular}{c} 
Bereich \\
\cline { 5 - 8 }
\end{tabular}} & & & \multicolumn{3}{|c|}{$\mathbf{N}(\%)$ der Isolate } \\
\hline Itraconazol & $\mathbf{N}$ & $\mathbf{M H K}_{\mathbf{5 0}}$ & $\mathbf{M H K}_{\mathbf{9 0}}$ & der MHKs & sensibel & d-sensibel & resistent \\
\hline Gesamt & 319 & 0,1 & $>16$ & $<0,0313->16$ & $176(55,2)$ & $25(7,8)$ & $118(37)$ \\
\hline C. albicans & 97 & 0 & 0,125 & $<0,0313->16$ & $90(92,8)$ & $2(2,1)$ & $5(5,1)$ \\
\hline C. glabrata & 104 & 16 & $>16$ & $<0,0313->16$ & $4(3,8)$ & $2(1,9)$ & $98(94,2)$ \\
\hline C. parapsilosis & 46 & 0,1 & 0,125 & $<0,0313-1$ & $42(91,3)$ & $3(6,5)$ & $1(2,2)$ \\
\hline C. tropicalis & 42 & 0,1 & 1 & $<0,0313->16$ & $27(64,3)$ & $9(21,4)$ & $6(14,3)$ \\
\hline C. kefyr & 7 & 0,1 & 0,0625 & $<0,0313-0,0625$ & $7(100)$ & - & - \\
\hline C. krusei & 7 & 0,5 & 8 & $0,125-8$ & $2(28,6 \%)$ & $3(42,8)$ & $2(28,6)$ \\
\hline
\end{tabular}

\subsubsection{Voriconazol}

\subsubsection{C. albicans}

Es wurden 98 C. albicans-Isolate mit dem Etest gegenüber Voriconazol getestet. Die MHKs lagen im Bereich der Konzentrationsstufen von $<0,0313 \mu \mathrm{g} / \mathrm{ml}$ bis $4 \mu \mathrm{g} / \mathrm{ml}$. Die $\mathrm{MHK}_{50}$ betrug $<0,0313 \mu \mathrm{g} / \mathrm{ml}$ und die $\mathrm{MHK}_{90} 0,0313 \mu \mathrm{g} / \mathrm{ml}$. 96 der Isolate (98\%) waren empfindlich. Jeweils ein Isolat (1\%) war den Kategorien dosisabhängig empfindlich sowie resistent zuzuordnen (Tabelle 4-15).

\subsubsection{C. glabrata}

Es wurden 103 C. glabrata-Stämme getestet. Die Hemmkonzentrationen lagen im Konzentrationsbereich zwischen $<0,0313 \mu \mathrm{g} / \mathrm{ml}$ und $>16 \mu \mathrm{g} / \mathrm{ml}$. Die $\mathrm{MHK}_{50}$ und MHK $_{90}$ betrugen $0,25 \mu \mathrm{g} / \mathrm{ml}$ und $2 \mu \mathrm{g} / \mathrm{ml}$. $79,6 \%$ (82 Stämme) waren empfindlich. $11,7 \%$ (zwölf Isolate) waren dosisabhängig empfindlich. Bei 8,7\% der Stämme (neun Isolaten) lag eine Voriconazol-Resistenz vor (Tabelle 4-15).

\subsubsection{C. parapsilosis}

Es wurden 45 C. parapsilosis-Isolate getestet. Die MHKs lagen innerhalb des Konzentrationsbereichs von $<0,0313 \mu \mathrm{g} / \mathrm{ml}$ bis $0,125 \mu \mathrm{g} / \mathrm{ml}$. Die $\mathrm{MHK}_{50}$ wurde bei einer Konzentration von $<0,0313 \mu \mathrm{g} / \mathrm{ml}$ und die $\mathrm{MHK}_{90}$ bei $0,0313 \mu \mathrm{g} / \mathrm{ml}$ bestimmt. Die Isolate waren durchgängig empfindlich (Tabelle 4-15). 


\subsubsection{C. tropicalis}

42 C. tropicalis-Isolate wurden getestet. Die MHKs lagen im Konzentrationsbereich von $<0,0313 \mu \mathrm{g} / \mathrm{ml}$ und $>16 \mu \mathrm{g} / \mathrm{ml}$. $\mathrm{MHK}_{50}$ und $\mathrm{MHK}_{90}$ betrugen $0,0313 \mu \mathrm{g} / \mathrm{ml}$ respektive $0,125 \mu \mathrm{g} / \mathrm{ml}$. Nur ein Stamm war Voriconazolresistent (2,4\%). Die anderen Isolate reagierten sensibel (97,6\%, Tabelle 4-15).

\subsubsection{C. kefyr}

Es wurden sieben C. kefyr-Isolate getestet. Die MHKs lagen dabei zwischen den Konzentrationsstufen $<0,0313 \mu \mathrm{g} / \mathrm{ml}$ und $0,0313 \mu \mathrm{g} / \mathrm{ml}$. Die $\mathrm{MHK}_{50}$ und $\mathrm{MHK}_{90}$ betrugen $<0,0313 \mu \mathrm{g} / \mathrm{ml}$ und $0,0313 \mu \mathrm{g} / \mathrm{ml}$. Alle Isolate waren empfindlich (Tabelle 4-15).

\subsubsection{C. krusei}

Acht C. krusei-Stämme wurden dem Etest unterzogen. Die MHKs lagen innerhalb des Konzentrationsbereichs $0,125 \mu \mathrm{g} / \mathrm{ml}$ bis $0,5 \mu \mathrm{g} / \mathrm{ml}$. $\mathrm{MHK}_{50}$ und $\mathrm{MHK}_{90}$ wurden bei $0,25 \mu \mathrm{g} / \mathrm{ml}$ sowie $0,5 \mu \mathrm{g} / \mathrm{ml}$ bestimmt. Alle Isolate waren Voriconazolempfindlich (Tabelle 4-15).

Die genaue Verteilung der Isolate über die unterschiedlichen Konzentrationsstufen ist Tabelle 7-4 (S. 99) zu entnehmen.

Tabelle 4-15: $\mathrm{MHK}_{50}$, $\mathrm{MHK}_{90}$ und die Bereiche der minimalen Hemmkonzentrationen (in $\mu \mathrm{g} / \mathrm{ml}$ ) sowie die Empfindlichkeit der häufigsten Spezies gegenüber Voriconazol

\begin{tabular}{|c|c|c|c|c|c|c|c|}
\hline \multirow[b]{2}{*}{ Voriconazol } & \multirow[b]{2}{*}{$\mathbf{N}$} & \multirow[b]{2}{*}{$\mathrm{MHK}_{50}$} & \multirow[b]{2}{*}{ MHK $_{90}$} & \multirow{2}{*}{$\begin{array}{c}\text { Bereich } \\
\text { der MHKs }\end{array}$} & \multicolumn{3}{|c|}{ N (\%) der Isolate } \\
\hline & & & & & sensibel & d-sensibel & resistent \\
\hline Gesamt & 319 & 0 & 0,5 & $<0,0313->16$ & $295(92,5)$ & $13(4,1)$ & $11(3,4)$ \\
\hline C. albicans & 98 & $<0,0313$ & 0,0313 & $<0,0313-4$ & $96(98)$ & $1(1)$ & $1(1)$ \\
\hline C. glabrata & 103 & 0,3 & 2 & $<0,0313->16$ & $82(79,6)$ & $12(11,7)$ & $9(8,7)$ \\
\hline C. parapsilosis & 45 & $<0,0313$ & 0,0313 & $<0,0313-0,125$ & $45(100)$ & - & - \\
\hline C. tropicalis & 42 & 0 & 0,125 & $<0,0313->16$ & $41(97,6)$ & - & $1(2,4)$ \\
\hline C. kefyr & 7 & $<0,0313$ & 0,0313 & $<0,0313-0,0313$ & $7(100)$ & - & - \\
\hline C. krusei & 8 & 0,3 & 0,5 & $0,125-0,5$ & $8(100)$ & - & - \\
\hline
\end{tabular}




\subsubsection{Caspofungin}

\subsubsection{C. albicans}

100 C. albicans-Isolate wurden dem Etest unterzogen; davon waren 99 Isolate $(99 \%)$ sensibel $(\leq 0,25 \mu \mathrm{g} / \mathrm{ml})$ und ein Isolat $(1 \%)$ intermediär sensibel $(0,5 \mu \mathrm{g} / \mathrm{ml})$. Die MHKs lagen im Konzentrationsbereich von $<0,0313 \mu \mathrm{g} / \mathrm{ml}$ bis $0,5 \mu \mathrm{g} / \mathrm{ml}$. Die $\mathrm{MHK}_{50}$ und $\mathrm{MHK}_{90}$ betrugen jeweils $0,125 \mu \mathrm{g} / \mathrm{ml}$ (Tabelle 4-16).

\subsubsection{C. glabrata}

Es wurden 101 C. glabrata-Isolate getestet. 100 Isolate (99\%) waren empfindlich $(\leq 0,125 \mu \mathrm{g} / \mathrm{ml})$, nur 1 Isolat $(1 \%)$ war intermediär empfindlich $(0,25 \mu \mathrm{g} / \mathrm{ml})$. Die MHKs lagen innerhalb des Bereichs der Konzentrationsstufen zwischen $<0,0313 \mu \mathrm{g} / \mathrm{ml}$ und $0,25 \mu \mathrm{g} / \mathrm{ml}$. Die $\mathrm{MHK}_{50}$ betrug $0,0625 \mu \mathrm{g} / \mathrm{ml}$ und die $\mathrm{MHK}_{90}$ $0,125 \mu \mathrm{g} / \mathrm{ml}$ (Tabelle 4-16).

\subsubsection{C. parapsilosis}

Alle 46 getesteten $C$. parapsilosis-Isolate waren empfindlich $(\leq 2 \mu \mathrm{g} / \mathrm{ml})$. Die MHKs lagen $\mathrm{im}$ Konzentrationsbereich von $0,125 \mu \mathrm{g} / \mathrm{ml}$ bis $2 \mu \mathrm{g} / \mathrm{ml}$. Die $\mathrm{MHK}_{50}$ und $\mathrm{MHK}_{90}$ betrugen $0,5 \mu \mathrm{g} / \mathrm{ml}$ und $1 \mu \mathrm{g} / \mathrm{ml}$ (Tabelle 4-16).

\subsubsection{C. tropicalis}

Es wurden 40 C. tropicalis-Isolate getestet. Ein Isolat $(2,5 \%)$ war intermediär empfindlich $(0,5 \mu \mathrm{g} / \mathrm{ml})$, alle anderen $(97,5 \%)$ waren empfindlich $(\leq 0,25 \mu \mathrm{g} / \mathrm{ml})$. Die MHKs lagen im Konzentrationsbereich von $<0,0313 \mu \mathrm{g} / \mathrm{ml}$ bis $0,5 \mu \mathrm{g} / \mathrm{ml}$. $\mathrm{MHK}_{50}$ und $\mathrm{MHK}_{90}$ wurden mit 0,0625 $\mathrm{gg} / \mathrm{ml}$ bzw. 0,125 $\mu \mathrm{g} / \mathrm{ml}$ berechnet (Tabelle 4-16).

\subsubsection{C. kefyr}

Die sieben $C$. kefyr-lsolate waren sensibel $(\leq 2 \mu \mathrm{g} / \mathrm{ml})$ gegenüber Caspofungin. Sie wiesen durchgängig eine $\mathrm{MHK}$ von $<0,0313 \mu \mathrm{g} / \mathrm{ml}$ auf. Die $\mathrm{MHK}_{50}$ und $\mathrm{MHK}_{90}$ betrugen damit ebenfalls jeweils $<0,0313 \mu \mathrm{g} / \mathrm{ml}$ (Tabelle 4-16).

\subsubsection{C. krusei}

Es wurden acht C. krusei-lsolate getestet, die alle Empfindlichkeit $(\leq 0,25 \mu \mathrm{g} / \mathrm{ml})$ zeigten. Die MHKs lagen im Bereich der Konzentrationsstufen zwischen $<0,0313 \mu \mathrm{g} / \mathrm{ml}$ bis $0,25 \mu \mathrm{g} / \mathrm{ml}$. $\mathrm{MHK}_{50}$ und $\mathrm{MHK}_{90}$ betrugen $0,125 \mu \mathrm{g} / \mathrm{ml}$ und $0,25 \mu \mathrm{g} / \mathrm{ml}$ (Tabelle 4-16).

Die genaue Verteilung der Isolate über die unterschiedlichen 
Konzentrationsstufen ist Tabelle 7-5 (S. 100) zu entnehmen.

Tabelle 4-16: $\mathrm{MHK}_{50}, \mathrm{MHK}_{90}$ und die Bereiche der minimalen Hemmkonzentrationen (in $\mu \mathrm{g} / \mathrm{ml}$ ) sowie die Empfindlichkeit der häufigsten Spezies gegenüber

Caspofungin

\begin{tabular}{|c|c|c|c|c|c|c|c|}
\hline \multirow[b]{2}{*}{ Caspofungin } & \multirow[b]{2}{*}{$\mathbf{N}$} & \multirow[b]{2}{*}{$\mathrm{MHK}_{50}$} & \multirow[b]{2}{*}{$\mathrm{MHK}_{90}$} & \multirow{2}{*}{$\begin{array}{c}\text { Bereich } \\
\text { der MHKs }\end{array}$} & \multicolumn{3}{|c|}{ N (\%) der Isolate } \\
\hline & & & & & sensibel & intermediär & resistent \\
\hline Gesamt & 318 & 0,1 & 0,5 & $<0,0313->16$ & $309(98,1)$ & $3(1)$ & $6(1,9)$ \\
\hline C. albicans & 100 & 0,1 & 0,125 & $<0,0313-0,5$ & $99(99)$ & $1(1)$ & - \\
\hline C. glabrata & 101 & 0,1 & 0,125 & $<0,0313-0,25$ & $100(99)$ & $1(1)$ & - \\
\hline C. parapsilosis & 46 & 0,5 & 1 & $0,125-2$ & $46100)$ & - & - \\
\hline C. tropicalis & 40 & 0,1 & 0,125 & $<0,0313-0,5$ & $39(97,5)$ & $1(2,5)$ & - \\
\hline C. kefyr & 7 & $<0,0313$ & $<0,0313$ & $<0,0313$ & $7(100)$ & - & - \\
\hline C. krusei & 8 & 0,1 & 0,25 & $<0,0313-0,25$ & $8(100)$ & - & - \\
\hline
\end{tabular}

\subsubsection{Amphotericin B}

\subsubsection{C. albicans}

Es sind 95 C. albicans-Isolate getestet worden. Die MHKs lagen im Bereich der Konzentrationsstufen zwischen $<0,0313 \mu \mathrm{g} / \mathrm{ml}$ und $0,25 \mu \mathrm{g} / \mathrm{ml}$. Die $\mathrm{MHK}_{50}$ und MHK $_{90}$ betrugen $0,0313 \mu \mathrm{g} / \mathrm{ml}$ bzw. $0,125 \mu \mathrm{g} / \mathrm{ml}$. Die Isolate waren durchgehend empfindlich gegenüber Amphotericin B (Tabelle 4-17).

\subsubsection{C. glabrata}

Es wurden 104 C. glabrata-Stämme mit dem Etest getestet. Die MHKs lagen im Bereich der Konzentrationsstufen zwischen $<0,0313 \mu \mathrm{g} / \mathrm{ml}$ und $1 \mu \mathrm{g} / \mathrm{ml}$. $\mathrm{MHK}_{50}$ und $\mathrm{MHK}_{90}$ lagen bei $0,0313 \mu \mathrm{g} / \mathrm{ml}$ und bei $0,125 \mu \mathrm{g} / \mathrm{ml}$. Die Isolate waren durchgängig empfindlich (Tabelle 4-17).

\subsubsection{C. parapsilosis}

46 C. parapsilosis-Isolate wurden getestet. Die MHKs lagen im Konzentrationsbereich zwischen $<0,0313 \mu \mathrm{g} / \mathrm{ml}$ bis $0,125 \mu \mathrm{g} / \mathrm{ml}$. Die $\mathrm{MHK}_{50}$ und $\mathrm{MHK}_{90}$ betrugen $0,0313 \mu \mathrm{g} / \mathrm{ml}$ und $0,125 \mu \mathrm{g} / \mathrm{ml}$. Alle Stämme waren AmphotericinB-empfindlich (Tabelle 4-17). 


\subsubsection{C. tropicalis}

42 C. tropicalis-Stämme wurden dem Etest unterzogen. Die MHKs lagen innerhalb des Bereichs von $<0,0313 \mu \mathrm{g} / \mathrm{ml}$ bis $0,5 \mu \mathrm{g} / \mathrm{ml}$. $\mathrm{MHK}_{50}$ und $\mathrm{MHK}_{90}$ wurden mit $<0,0313 \mu \mathrm{g} / \mathrm{ml}$ und $0,125 \mu \mathrm{g} / \mathrm{ml}$ bestimmt. Die Isolate waren durchgängig empfindlich (Tabelle 4-17).

\subsubsection{C. kefyr}

Sieben C. kefyr-Isolate wurden mit dem Etest getestet. Die MHKs lagen im Bereich von $0,125 \mu \mathrm{g} / \mathrm{ml}$ bis $0,25 \mu \mathrm{g} / \mathrm{ml}$. Sowohl die $\mathrm{MHK}_{50}$ als auch die $\mathrm{MHK}_{90}$ lagen bei $0,25 \mu \mathrm{g} / \mathrm{ml}$. Die Stämme waren alle empfindlich (Tabelle 4-17).

\subsubsection{C. krusei}

Acht C. krusei-Stämme wurden getestet. Die MHKs lagen im Bereich der Konzentrationsstufen zwischen $<0,0313 \mu \mathrm{g} / \mathrm{ml}$ und $0,25 \mu \mathrm{g} / \mathrm{ml}$. Die $\mathrm{MHK}_{50}$ und $\mathrm{MHK}_{90}$ betrugen $0,0625 \mu \mathrm{g} / \mathrm{ml}$ und $0,25 \mu \mathrm{g} / \mathrm{ml}$. Sie wiesen durchgängige Empfindlichkeit gegenüber Amphotericin B auf (Tabelle 4-17).

Die genaue Verteilung der Isolate über die unterschiedlichen Konzentrationsstufen ist Tabelle 7-6 (S. 101) zu entnehmen.

Tabelle 4-17: $\mathrm{MHK}_{50}, \mathrm{MHK}_{90}$ und die Bereiche der minimalen Hemmkonzentrationen (in $\mu \mathrm{g} / \mathrm{ml}$ ) sowie die Empfindlichkeit der häufigsten Spezies gegenüber

Amphotericin B

\begin{tabular}{|l|c|c|c|c|c|c|}
\hline & & & & \multicolumn{2}{|c|}{ Bereich } & \multicolumn{2}{|c|}{ N (\%) der Isolate } \\
\cline { 5 - 7 } Amphotericin B & N & MHK $_{\mathbf{5 0}}$ & MHK $_{\mathbf{9 0}}$ & der MHKs & sensibel & resistent \\
\hline Gesamt & 318 & 0 & 0,125 & $<0,0313-1$ & $318(100)$ & - \\
\hline C. albicans & 95 & 0 & 0,125 & $<0,0313-0,25$ & $95(100)$ & - \\
\hline C. glabrata & 104 & 0 & 0,125 & $<0,0313-1$ & $104(100)$ & - \\
\hline C. parapsilosis & 46 & 0 & 0,125 & $<0,0313-0,125$ & $46(100)$ & - \\
\hline C. tropicalis & 42 & $<0,0313$ & 0,125 & $<0,0313-0,5$ & $42(100)$ & - \\
\hline C. kefyr & 7 & 0,3 & 0,25 & $0,125-0,25$ & $7(100)$ & - \\
\hline C. krusei & 8 & 0,1 & 0,25 & $<0,0313-0,25$ & $8(100)$ & - \\
\hline
\end{tabular}




\subsubsection{5-Fluorcytosin}

\subsubsection{C. albicans}

Alle 322 C. albicans-Isolate wurden mit dem Etest gegenüber 5-Fluorcytosin getestet. Die MHKs lagen im Bereich von $<0,125 \mu \mathrm{g} / \mathrm{ml}$ bis $>16 \mu \mathrm{g} / \mathrm{ml}$. Die $\mathrm{MHK}_{50}$ betrug $<0,125 \mu \mathrm{g} / \mathrm{ml}$ und die $\mathrm{MHK}_{90} 0,125 \mu \mathrm{g} / \mathrm{ml}$. 99,4\% (515 Isolate) von $C$. albicans waren empfindlich. $0,6 \%$ (zwei Isolate) erwiesen sich als resistent (Tabelle 4-18).

\subsubsection{C. glabrata}

Es wurden 105 C. glabrata-Stämme getestet. Die MHKs lagen innerhalb des Bereichs der Konzentrationsstufen von $<0,125 \mu \mathrm{g} / \mathrm{ml}$ bis $8 \mu \mathrm{g} / \mathrm{ml}$. Die $\mathrm{MHK}_{50}$ und $\mathrm{MHK}_{90}$ betrugen $<0,125 \mu \mathrm{g} / \mathrm{ml}$ sowie $0,125 \mu \mathrm{g} / \mathrm{ml}$. Die Stämme waren mit $99,1 \%$ (104 Isolate) fast durchgehend empfindlich. Nur ein Stamm (0,9\%) war intermedär empfindlich (Tabelle 4-18).

\subsubsection{C. parapsilosis}

Es wurden 46 C. parapsilosis-Isolate getestet. Die MHKs lagen im Konzentrationsbereich von $<0,125 \mu \mathrm{g} / \mathrm{ml}$ bis $8 \mu \mathrm{g} / \mathrm{ml}$. Die $\mathrm{MHK}_{50}$ wurde bei $<0,125 \mu \mathrm{g} / \mathrm{ml}$ und die $\mathrm{MHK}_{90}$ bei $0,25 \mu \mathrm{g} / \mathrm{ml}$ ermittelt. $97,8 \%$ (45 Isolate) waren empfindlich, und 2,2\% (ein Isolat) waren intermediär empfindlich (Tabelle 4-18).

\subsubsection{C. tropicalis}

42 C. tropicalis-Stämme wurden der Testung unterzogen. Die MHKs lagen im Bereich von $<0,125 \mu \mathrm{g} / \mathrm{ml}$ bis $>16 \mu \mathrm{g} / \mathrm{ml}$. Sowohl die $\mathrm{MHK}_{50}$ als auch die $\mathrm{MHK}_{90}$ wurden mit $>16 \mu \mathrm{g} / \mathrm{ml}$ berechnet. 59,5\% (25 Isolate) waren resistent. Demgegenüber standen 17 (40,5\%) empfindliche Stämme (Tabelle 4-18).

\subsubsection{C. kefyr}

Elf C. kefyr-Isolate wurden dem Etest unterzogen. Die MHKs lagen im Konzentrationsbereich zwischen $<0,125 \mu \mathrm{g} / \mathrm{ml}$ bis $>16 \mu \mathrm{g} / \mathrm{ml}$. Die $\mathrm{MHK}_{50}$ bzw. $\mathrm{MHK}_{90}$ betrugen $16 \mu \mathrm{g} / \mathrm{ml}$ respektive $>16 \mu \mathrm{g} / \mathrm{ml} .36,4 \%$ der Isolate (vier Stämme) waren empfindlich. Als intermediär empfindlich waren $27,2 \%$ (drei Stämme) und als resistent 36,4\% (vier Stämme) einzuordnen (Tabelle 4-18). 


\subsubsection{C. krusei}

Acht C. krusei-Stämme wurden mit dem Etest getestet. Die MHKs lagen im Bereich der Konzentrationsstufen zwischen $0,125 \mu \mathrm{g} / \mathrm{ml}$ und $>16 \mu \mathrm{g} / \mathrm{ml}$. Sowohl die $\mathrm{MHK}_{50}$ als auch die $\mathrm{MHK}_{90}$ betrugen $>16 \mu \mathrm{g} / \mathrm{ml}$. Nur ein Isolat war empfindlich $(12,5 \%)$. Die restlichen sieben Isolate $(87,5 \%)$ waren durchweg resistent (Tabelle 4-18).

Die genaue Verteilung der Isolate über die unterschiedlichen Konzentrationsstufen ist Tabelle 7-7 (S. 102) zu entnehmen.

Tabelle 4-18: $\mathrm{MHK}_{50}, \mathrm{MHK}_{90}$ und die Bereiche der minimalen Hemmkonzentrationen (in $\mu \mathrm{g} / \mathrm{ml}$ ) sowie die Empfindlichkeit der häufigsten Spezies gegenüber 5Fluorcytosin

\begin{tabular}{|c|c|c|c|c|c|c|c|}
\hline \multirow[b]{2}{*}{ 5-Fluorcytosin } & \multirow[b]{2}{*}{$\mathbf{N}$} & \multirow[b]{2}{*}{$\mathrm{MHK}_{50}$} & \multirow[b]{2}{*}{$\mathrm{MHK}_{90}$} & \multirow{2}{*}{$\begin{array}{c}\text { Bereich } \\
\text { der MHKs }\end{array}$} & \multicolumn{3}{|c|}{ N (\%) der Isolate } \\
\hline & & & & & sensibel & intermed. & resistent \\
\hline Gesamt & 567 & $<0,125$ & 2 & $<0,125->16$ & $515(90,8)$ & $6(1,1)$ & $46(8,1)$ \\
\hline C. albicans & 322 & $<0,125$ & 0,125 & $<0,125->16$ & $320(99,4)$ & - & $2(0,6)$ \\
\hline C. glabrata & 105 & $<0,125$ & 0,125 & $<0,125-8$ & $104(99,1)$ & $1(0,9)$ & - \\
\hline C. parapsilosis & 46 & $<0,125$ & 0,25 & $<0,125-8$ & $45(97,8 \%)$ & $1(2,2 \%)$ & - \\
\hline C. tropicalis & 42 & $>16$ & $>16$ & $<0,125->16$ & $17(40,5)$ & - & $25(59,5)$ \\
\hline C. kefyr & 11 & 16 & $>16$ & $<0,125->16$ & $4(36,4 \%)$ & $3(27,2 \%)$ & $4(36,4)$ \\
\hline C. krusei & 8 & $>16$ & $>16$ & $0,125->16$ & $1(12,5)$ & - & $7(87,5)$ \\
\hline
\end{tabular}

\subsubsection{Zusammenfassung aller Etest-Ergebnisse}

Gegenüber Fluconazol und 5-Fluorcytosin wurden alle Isolate mit dem Etest getestet. Des Weiteren wurden die Isolate, die hierbei durch herabgesetzte Empfindlichkeit auffielen, sowie alle C. glabrata-Isolate gegenüber den anderen vier Antimykotika getestet. Gegenüber Fluconazol und insbesondere Itraconazol standen die Spezies C. glabrata und $C$. kusei unter den Isolaten mit herabgesetzter Empfindlichkeit im Vordergrund. Der Anteil der Isolate mit herabgesetzter Empfindlichkeit war bei Voriconazol deutlich niedriger als bei den anderen Azolen. C. glabrata wies dennoch die höchste Resistenzrate auf. Die 
Wirksamkeit von Caspofungin war gegenüber der großen Mehrzahl der Isolate hervorragend. Ausnahme war nur ein C. glabrata-Isolat und die selteneren Spezies Cr. neoformans, G. capitatum sowie Trichosporon spp.. Alle getesteten Isolate waren gegenüber Amphotericin $B$ empfindlich. Bei insgesamt hoher Empfindlichkeit von $90,8 \%$ gegenüber 5-Fluorcytosin wiesen die Isolate der Spezies C. krusei und C. tropicalis hohe Resistenzraten auf.

Die Etest-Ergebnisse für die einzelnen Antimykotika werden in der nachstehenden Tabelle 4-19 noch einmal für die jeweils getesteten Isolate aufgeführt.

Tabelle 4-19: Vergleich der $\mathrm{MHK}_{50}$ und $\mathrm{MHK}_{90}$, den Bereichen der MHKs (in $\mu \mathrm{g} / \mathrm{ml}$ ) sowie der kategorialen Bewertung gegenüber den sechs verwendeten Antimykotika, angegeben für alle Isolate, die dem Etest unterzogen wurden

\begin{tabular}{|l|c|c|c|c|c|c|c|}
\hline & & & & & \multicolumn{3}{|c|}{ Anzahl (\%) der Isolate } \\
\cline { 6 - 9 } & & & & & & intermediär/ & \\
Antimykotikum & $\mathbf{N}$ & MHK $_{50}$ & MHK $_{90}$ & Bereich der MHKs & sensibel & d-sensibel & resistent \\
\hline Fluconazol & 567 & 0,5 & 16 & $<0,25->32$ & $466(82,2)$ & $69(12,2)$ & $32(5,6)$ \\
\hline Itraconazol & 319 & 0,125 & $>16$ & $<0,0313->16$ & $176(55,2)$ & $25(7,8)$ & $118(37)$ \\
\hline Voriconazol & 319 & 0,0313 & 0,5 & $<0,0313->16$ & $295(92,5)$ & $13(4,1)$ & $11(3,4)$ \\
\hline Caspofungin & 318 & 0,125 & 0,5 & $<0,0313->16$ & $309(98,1)$ & $3(1)$ & $6(1,9)$ \\
\hline Amphotericin B & 318 & 0,0313 & 0,125 & $<0,0313-1$ & $318(100)$ & - & \\
\hline 5-Fluorcytosin & 567 & $<0,125$ & 2 & $<0,125->16$ & $515(90,8)$ & $6(1,1)$ & $46(8,1)$ \\
\hline
\end{tabular}

\subsection{Vergleich von Mikrodilutionstest und Etest}

Die Korrelation der Ergebnisse der beiden Testmethoden erfolgte wie im Abschnitt 3.7 beschrieben. Die im folgenden Abschnitt als "höher“ bezeichneten Ergebnisse haben eine höhere MHK und sind somit als weniger empfindlich einzustufen. Umgekehrtes gilt für Ergebnisse, die als „niedriger“ bezeichnet werden.

Analog zu den vorherigen Abschnitten werden für die verschiedenen 
Antimykotika jeweils die Ergebnisse der Übereinstimmung der sechs häufigsten Spezies einzeln sowie die aller getesteten Isolate gemeinsam aufgelistet. Die selteneren Spezies sind nicht separat aufgeführt.

Dabei ist zu beachten, dass, wie im Abschnitt 4.3 beschrieben, alle 567 Isolate mit dem Etest gegenüber Fluconazol und 5-Fluorcytosin getestet wurden. Da nur Isolate, die hierbei eine herabgesetzte Empfindlichkeit aufwiesen sowie alle $C$. glabrata-Isolate, auch gegenüber den anderen vier Antimykotika getestet wurden, liegt somit nur dieser Anteil der Isolate zum Vergleich der beiden Testmethoden vor.

\subsubsection{Fluconazol}

\subsubsection{C. albicans}

Für die Korrelation lagen 322 C. albicans-Isolate vor. Die Übereinstimmung der Ergebnisse der Konzentrationsstufen lag bei 92,5\%. 98,5\% wurden durch beide Testverfahren der gleichen Kategorie zugeordnet (Tabelle 4-20).

\subsubsection{C. glabrata}

Es lagen 105 C. glabrata-Isolate für die Korrelation vor. Die Übereinstimmung der Konzentrationsstufen war mit $86,7 \%$ am niedrigsten im Vergleich zu den anderen Spezies. Die kategorialen Ergebnisse deckten sich in nur 43,8\% (Tabelle 4-20).

\subsubsection{C. parapsilosis}

Die Übereinstimmung wurde für 46 C. parapsilosis-Isolate ermittelt. Die Konzentrationsstufen stimmten zu 95,6\% überein. Hinsichtlich der Kategorien stimmten die Ergebnisse in allen Fällen überein (Tabelle 4-20).

\subsubsection{C. tropicalis}

Die Korrelation erfolgte für 42 C. tropicalis-Stämme. Die Übereinstimmung bezüglich der Konzentrationsstufen lag bei 90,5\%. Die kategoriale Übereinstimmung war mit 95,2\% höher (Tabelle 4-20).

\subsubsection{C. kefyr}

Es lagen elf $C$. kefyr-Isolate für die Korrelation vor. Die Ergebnisse stimmten sowohl bezüglich der Konzentrationsstufen als auch der Kategorien in allen Fällen überein (Tabelle 4-20). 


\subsubsection{C. krusei}

Die Korrelation erfolgte für acht C. krusei-Isolate. Während die Ergebnisse der Konzentrationsstufen vollständige Deckung aufwiesen, stimmten die Ergebnisse der Kategorisierung nur in 37,5\% der Fälle überein (Tabelle 4-20).

\subsubsection{Zusammenfassung}

Die Korrelation wurde für die gesamten 567 Isolate bestimmt. Die Übereinstimmung der Konzentrationsstufen lag bei 91,4\%. Die Übereinstimmung bezüglich der Kategorien war mit $85,2 \%$ geringer.

Die Übereinstimmung war bei C. glabrata sowohl bezüglich der Konzentrationsstufen als auch der Kategorien am niedrigsten. Bei abweichenden Ergebnissen lieferte der Etest höhere Ergebnisse als die Mikrodilution (Tabelle 420).

Verglich man die Korrelation der Ergebnisse aller empfindlichen Isolate mit der Korrelation aller Isolate mit herabgesetzter Empfindlichkeit, so fiel eine deutliche Diskrepanz auf. Die empfindlichen Isolate zeichneten sich durch eine hohe Übereinstimmung von $94,1 \%$ bei den Konzentrationsstufen sowie $100 \%$ in den Kategorien aus. Die resistenten Stämme dagegen wiesen eine deutlich schlechtere Deckung von 80,9\% bezüglich der Konzentrationsstufen und lediglich $25,5 \%$ bezüglich der Kategorien auf.

Tabelle 4-20: Prozentuale Korrelation der beiden Testmethoden bezüglich der Konzentrationsstufen (MHK-Stufen) und der Bewertungskategorie sowie der Anteil der diskrepanten Ergebnisse mit höherem Etest-Ergebnis (E>M) gegenüber Fluconazol

\begin{tabular}{|l|c|c|c|c|}
\hline \multirow{2}{*}{ Fluconazol } & & \multicolumn{2}{|c|}{ Korrelation in \% } & \multirow{2}{*}{ N (\%) } \\
\cline { 3 - 4 } & N & MHK & Kategorie & E>M \\
\hline Gesamt & 567 & 91,4 & 85,2 & $32(5,6)$ \\
\hline C. albicans & 322 & 92,5 & 98,5 & $14(4,3)$ \\
\hline C. glabrata & 105 & 86,7 & 43,8 & $12(11,4)$ \\
\hline C. parapsilosis & 46 & 95,6 & 100 & - \\
\hline C. tropicalis & 42 & 90,5 & 95,2 & $2(4,8)$ \\
\hline C. kefyr & 11 & 100 & 100 & - \\
\hline C. krusei & 8 & 100 & 37,5 & - \\
\hline
\end{tabular}




\subsubsection{Itraconazol}

\subsubsection{C. albicans}

Die Übereinstimmung wurde für 97 C. albicans-Isolate bestimmt. Bezüglich der Konzentrationsstufen lag die Deckung bei 60,2\%. Die kategoriale Übereinstimmung lag bei 68,4\% (Tabelle 4-21).

\subsubsection{C. glabrata}

Die Korrelation wurde für 104 C. glabrata-Isolate ermittelt. Die Übereinstimmung bezüglich der Konzentrationsstufen lag bei 25,7\%. Dies war im Vergleich zu den anderen Spezies das niedrigste Ergebnis. Die kategoriale Deckung war in 59\% gegeben (Tabelle 4-21).

\subsubsection{C. parapsilosis}

Grundlage für die Korrelation waren 46 C. parapsilosis-Isolate. Die Übereinstimmung bezüglich der Konzentrationsstufen lag bei $89,1 \%$. Hinsichtlich der Kategorien zeigte sich eine Deckung von 76,1\% (Tabelle 4-21).

\subsubsection{C. tropicalis}

Die Korrelation wurde für 42 C. tropicalis-Stämmen bestimmt. Übereinstimmende Ergebnisse lagen in $69,1 \%$ vor. Die kategoriale Deckung wurde mit 57,1\% bestimmt (Tabelle 4-21).

\subsubsection{C. kefyr}

Sieben C. kefyr-Isolate wurden mit beiden Testverfahren getestet. Eine Übereinstimmung der Konzentrationsstufen lag in 57,1\% vor. In Hinblick auf die Kategorien stimmten die Ergebnisse der beiden Testmethoden in keinem Fall überein. Die Mikrodilution erbrachte in allen Fällen höhere Ergebnisse als der Etest (Tabelle 4-21).

\subsubsection{C. krusei}

Die Korrelation wurde für sieben C. krusei-Isolate ermittelt. In 71,4\% deckten sich die Ergebnisse der beiden Testverfahren bezüglich der Konzentrationsstufen. Die kategoriale Übereinstimmung lag bei 42,9\% (Tabelle 4-21).

\subsubsection{Zusammenfassung}

319 Isolate dienten als Grundlage der Korrelation. Bezüglich der Kategorien lag die Übereinstimmung bei $63,3 \%$ und bezüglich der Konzentrationsstufen bei 
55,5\%. Hervorzuheben ist im Hinblick auf die Konzentrationsstufen die niedrige Korrelation bei C. glabrata $(25,7 \%)$. Bei $C$. kefyr $(57,1 \%)$ und C. albicans $(60,2 \%)$ war die Deckung höher. Mit $89,1 \%$ wurde die beste Übereinstimmung bei $C$. parapsilosis erzielt. Die Übereinstimmung bei Itraconazol ist niedriger als bei den anderen Antimykotika. Auch bei Itraconazol erbrachte der Etest bei abweichenden Ergebnissen häufiger höhere Ergebnisse als die Mikrodilution (Tabelle 4-21).

Tabelle 4-21: Prozentuale Korrelation der beiden Testmethoden bezüglich der Konzentrationsstufen (MHK-Stufen) und der Bewertungskategorie sowie der Anteil der diskrepanten Ergebnisse mit höherem Etest-Ergebnis $(E>M)$ gegenüber Itraconazol, angegeben für alle Isolate der häufigsten Spezies, die dem Etest unterzogen wurden

\begin{tabular}{|l|c|c|c|c|}
\hline \multirow{2}{*}{ Itraconazol } & \multirow{2}{*}{ N } & \multicolumn{2}{|c|}{ Korrelation in \% } & \multirow{2}{*}{ N (\%) } \\
\cline { 3 - 4 } Gesamt & 319 & 55,5 & Kategorie & E>M \\
\hline C. albicans & 97 & 60,2 & 63,3 & $83(26)$ \\
\hline C. glabrata & 104 & 25,7 & 68,4 & $5(5,1)$ \\
\hline C. parapsilosis & 46 & 89,1 & 59 & $74(70,5)$ \\
\hline C. tropicalis & 42 & 69,1 & 76,1 & - \\
\hline C. kefyr & 7 & 57,1 & 57,1 & $1(2,4)$ \\
\hline C. krusei & 7 & 71,4 & - & - \\
\hline
\end{tabular}

\subsubsection{Voriconazol}

\subsubsection{C. albicans}

Die Korrelation wurde für 98 C. albicans-Isolate bestimmt. Die Übereinstimmung in Hinblick auf die Konzentrationsstufen lag bei $79 \%$. Die kategoriale Übereinstimmung war mit 83\% etwas höher (Tabelle 4-22).

\subsubsection{C. glabrata}

Grundlage für die Korrelation waren 103 C. glabrata-Isolate. Hinsichtlich der Konzentrationsstufen stimmten die Ergebnisse in $85,4 \%$ überein. Eine kategoriale Übereinstimmung lag bei $88,3 \%$ der Fälle vor (Tabelle 4-22). 


\subsubsection{C. parapsilosis}

45 C. parapsilosis-Stämme wurden beiden Testverfahren unterzogen. Vollständige Deckung der Ergebnisse lag sowohl bezüglich der Konzentrationsstufen als auch der Kategorien vor (Tabelle 4-22).

\subsubsection{C. tropicalis}

Die Korrelation wurde für 42 C. tropicalis-Stämme ermittelt. Die Ergebnisse der Konzentrationsstufen stimmten zu 85,7\% überein. Die Deckung der Ergebnisse hinsichtlich der Kategorien lag bei 88,1\% (Tabelle 4-22).

\subsubsection{C. kefyr}

Sieben $C$. kefyr-Isolate wurden für die Korrelation herangezogen. Alle Ergebnisse sowohl im Hinblick auf die Konzentrationsstufen als auch auf die Kategorien stimmten überein (Tabelle 4-22).

\subsubsection{C. krusei}

Die Korrelation wurde für acht $C$. krusei-Stämme bestimmt. Es wurde eine Deckung der Konzentrationsstufen in $87,5 \%$ beobachtet. Hinsichtlich der Kategorien war eine vollständige Übereinstimmung gegeben (Tabelle 4-22).

\subsubsection{Zusammenfassung}

Die Korrelation wurde bezüglich Voriconazol für 319 Isolate durchgeführt. Die Deckung der Ergebnisse der Kategorien lag für alle Isolate bei $90 \%$ und bezüglich der Konzentrationsbereiche bei $86,2 \%$. Für C. albicans war die Korrelation am schlechtesten. Bei Abweichungen der Ergebnisse waren häufiger höhere Ergebnisse der Mikrodilution als des Etests zu vermerken (Tabelle 4-22). 
Tabelle 4-22: Prozentuale Korrelation der beiden Testmethoden bezüglich der Konzentrationsstufen (MHK-Stufen) und der Bewertungskategorie sowie der Anteil der diskrepanten Ergebnisse mit höherem Etest-Ergebnis $(E>M)$ gegenüber Voriconazol, angegeben für alle Isolate der häufigsten Spezies, die dem Etest unterzogen wurden

\begin{tabular}{|l|c|c|c|c|}
\hline \multirow{2}{*}{ Voriconazol } & N & \multicolumn{2}{|c|}{ Korrelation in \% } & \multirow{2}{*}{ N (\%) } \\
\cline { 3 - 4 } & EHK & Kategorie & $16(5)$ \\
\hline Gesamt & 319 & 86,2 & 90 & $2(2)$ \\
\hline C. albicans & 98 & 79 & 83 & $12(11,6)$ \\
\hline C. glabrata & 103 & 85,4 & 88,3 & - \\
\hline C. parapsilosis & 45 & 100 & 100 & $1(2,4)$ \\
\hline C. tropicalis & 42 & 85,7 & 88,1 & - \\
\hline C. kefyr & 7 & 100 & 100 & - \\
\hline C. krusei & 8 & 87,5 & 100 & \\
\hline
\end{tabular}

\subsubsection{Caspofungin}

\subsubsection{C. albicans}

Es lagen 100 C. albicans-Isolate zur Korrelation vor. Die Übereinstimmung hinsichtlich der Konzentrationsstufen lag bei $75,5 \%$. Diese Korrelation ist im Vergleich zu allen anderen Spezies am niedrigsten. Die kategoriale Übereinstimmung war mit 99\% hervorragend (Tabelle 4-23).

\subsubsection{C. glabrata}

Die Korrelation wurde für 101 C. glabrata-lsolate bestimmt. Eine Übereinstimmung der MHK-Ergebnisse lag in 95,1\% und der kategorialen Ergebnisse in $94,1 \%$ vor (Tabelle 4-23).

\subsubsection{C. parapsilosis}

Es wurden 46 C. parapsilosis-Isolate beiden Testverfahren unterzogen. Die MHK-Ergebnisse waren zu 93,5\% identisch, kategorial zu 100\% (Tabelle 4-23).

\subsubsection{C. tropicalis}

Die Korrelation wurde bei 40 C. tropicalis-Stämmen durchgeführt. Die Ergebnisse in Bezug auf die Konzentrationsstufen stimmten in $85 \%$ der Fälle überein, in Bezug auf die Kategorien in 97,5\% (Tabelle 4-23). 


\subsubsection{C. kefyr}

Sieben C. kefyr-Isolate wurden mit beiden Testverfahren getestet. Die Korrelation betrug in beiden Fällen 100\% (Tabelle 4-23).

\subsubsection{C. krusei}

Es lagen acht C. krusei-Stämme zur Korrelation vor. Die Deckung der MHKErgebnisse war in $87,5 \%$ der Fälle gegeben, die der kategorialen Ergebnisse in 100\% der Fälle (Tabelle 4-23).

\subsubsection{Zusammenfassung}

Die Korrelation der Ergebnisse wurde für 318 Isolate bestimmt, wobei die Übereinstimmung hinsichtlich der MHKs bei $87,4 \%$ lag und in Bezug auf die Kategorien 97,5\%. Bei Abweichungen der Ergebnisse der Konzentrationsstufen war auch hier zu beobachten, dass der Etest häufig höhere Konzentrationsbereiche maß als die Mikrodilution (Tabelle 4-23).

Tabelle 4-23: Prozentuale Korrelation der beiden Testmethoden bezüglich der Konzentrationsstufen (MHK-Stufen) und der Bewertungskategorie sowie der Anteil der diskrepanten Ergebnisse mit höherem Etest-Ergebnis $(E>M)$ gegenüber Caspofungin, angegeben für alle Isolate der häufigsten Spezies, die dem Etest unterzogen wurden

\begin{tabular}{|l|c|c|c|c|}
\hline \multirow{2}{*}{ Caspofungin } & \multirow{2}{*}{$\mathbf{N}$} & \multicolumn{2}{|c|}{ Korrelation in \% } & N (\%) \\
\cline { 3 - 5 } & 318 & MHK & Kategorie & E>M \\
\hline Gesamt & 100 & 75,4 & 97,5 & $33(10,4)$ \\
\hline C. albicans & 101 & 95,1 & 99 & $24(24,2)$ \\
\hline C. glabrata & 46 & 93,5 & 94,1 & $2(2)$ \\
\hline C. tropicalis & 40 & 85 & 100 & $3(6,5)$ \\
\hline C. kefyr & 7 & 100 & 97,5 & $4(10)$ \\
\hline C. krusei & 8 & 87,5 & 100 & - \\
\hline
\end{tabular}




\subsubsection{Amphotericin B}

Für Amphotericin B wurde nur die Übereinstimmung der Kategorien geprüft, da eine Kumulation der Ergebnisse der Mikrodilution bei wenigen Konzentrationsstufen bekannt ist, die des Etests jedoch eine größere Bandbreite aufweisen (NCCLS M27-A2 2002, Park BJ et al. 2006, Rex et al. 1995, vgl. auch Abschnitt 4.2.7 sowie 4.3.6).

\subsubsection{C. albicans}

Es wurden 95 Isolate beiden Testverfahren unterzogen. In allen Fällen lag eine vollständige Deckung vor (Tabelle 4-24).

\subsubsection{C. glabrata}

104 Isolate wurden getestet. In allen Fällen lag eine vollständige Deckung vor (Tabelle 4-24).

\subsubsection{C. parapsilosis}

46 Stämme wurden beiden Testverfahren unterzogen. Auch hier lag eine 100\%ige Übereinstimmung der Ergebnisse vor (Tabelle 4-24).

\subsubsection{C. tropicalis}

42 C. tropicalis-Isolate waren Grundlage der Korrelation. Eine vollständige Deckung der Ergebnisse war in allen Fällen gegeben (Tabelle 4-24).

\subsubsection{C. kefyr}

Sieben Stämme von C. kefyr wurden getestet. Beide Testverfahren erzielten in allen Fällen dieselben Ergebnisse (Tabelle 4-24).

\subsubsection{C. krusei}

Acht C. krusei-Isolate wurden beiden Testverfahren unterzogen. Alle Ergebnisse stimmten überein (Tabelle 4-24).

\subsubsection{Zusammenfassung}

Es wurden insgesamt 318 Isolate beiden Testverfahren unterzogen. Alle Ergebnisse stimmten für Amphotericin B überein (Tabelle 4-24). 
Tabelle 4-24: Prozentuale Korrelation der beiden Testmethoden bezüglich der Bewertungskategorie gegenüber Amphotericin B, angegeben für alle Isolate der häufigsten Spezies, die dem Etest unterzogen wurden

\begin{tabular}{|l|c|c|}
\hline Amphotericin B & N & $\begin{array}{c}\text { Korrelation in \% } \\
\text { Kategorie }\end{array}$ \\
\hline Gesamt & 318 & 100 \\
\hline C. albicans & 100 & 100 \\
\hline C. glabrata & 101 & 100 \\
\hline C. parapsilosis & 46 & 100 \\
\hline C. tropicalis & 40 & 100 \\
\hline C. kefyr & 7 & 100 \\
\hline C. krusei & 8 & 100 \\
\hline
\end{tabular}

\subsubsection{5-Fluorcytosin}

\subsubsection{C. albicans}

322 C. albicans-Isolate wurden beiden Testmethoden unterzogen. Die Ergebnisse stimmten bezüglich der Konzentrationsstufen in $96,6 \%$ überein. In 99,1\% stimmte auch die Zuordnung zu den Kategorien empfindlich, intermediär sowie resistent überein (Tabelle 4-25).

\subsubsection{C. glabrata}

Die Korrelation erfolgte anhand 105 C. glabrata-Isolaten. Den gleichen Konzentrationsstufen wurden $96,2 \%$ zugeordnet. Nur bei einem Isolat lag keine kategoriale Übereinstimmung vor (99,1\% Übereinstimmung, Tabelle 4-25).

\subsubsection{C. parapsilosis}

Es wurden 46 C. parapsilosis-Isolate zur Korrelation herangezogen. Alle Ergebnisse der Konzentrationsstufen stimmten überein. Bezüglich der Kategorien lag die Übereinstimmung bei 97,8\% (Tabelle 4-25).

\subsubsection{C. tropicalis}

Zur Korrelation wurden 42 C. tropicalis-Stämme betrachtet. Die Übereinstimmung bezüglich der Konzentrationsstufen lag bei 92,9\%. Die kategoriale Übereinstimmung lag bei 90,5\% (Tabelle 4-25). 


\subsubsection{C. kefyr}

Für die Korrelation der C. kefyr-Isolate lagen die Ergebnisse von elf Isolaten vor. Bezüglich der Konzentrationsstufen stimmten die Ergebnisse in 18,2\% (zwei Isolate) überein. In $36,4 \%$ (vier Stämme) deckte sich die kategoriale Zuordnung (Tabelle 4-25).

\subsubsection{C. krusei}

Die Korrelation wurde für acht C. krusei-Isolate ermittelt. Die Übereinstimmung der Konzentrationsstufen lag bei $87,5 \%$. Eine kategoriale Übereinstimmung war in $25 \%$ gegeben (Tabelle $4-25$ ).

\subsubsection{Zusammenfassung}

Die Korrelation wurde für die gesamten 567 Isolate ermittelt. Die Übereinstimmung der Konzentrationsstufen war mit 94,9\% höher als die kategoriale Übereinstimmung (93,8\%). In den Fällen, in denen die Ergebnisse voneinander abwichen, waren die Etest-Ergebnisse höher als die Ergebnisse der Mikrodilution. Am geringsten war die Übereinstimmung der Ergebnisse bei $C$. kefyr (36,4\%, Tabelle 4-25).

Tabelle 4-25: Prozentuale Korrelation der beiden Testmethoden bezüglich der Konzentrationsstufen (MHK-Stufen) und der Bewertungskategorie sowie der Anteil der diskrepanten Ergebnisse mit höherem Etest-Ergebnis $(E>M)$ gegenüber 5-Fluorcytosin

\begin{tabular}{|l|c|c|c|c|}
\hline \multirow{2}{*}{ 5-Fluorcytosin } & \multirow{2}{*}{$\mathbf{N}$} & \multicolumn{2}{|c|}{ Korrelation in \% } & \multirow{2}{*}{ N (\%) } \\
\cline { 3 - 4 } Gesamt & 567 & 93,8 & Kategorie & E>M \\
\hline C. albicans & 322 & 96,6 & 94,9 & $30(5,3)$ \\
\hline C. glabrata & 105 & 96,2 & 99,1 & $10(3,1)$ \\
\hline C. parapsilosis & 46 & 100 & 99,1 & $4(3,8)$ \\
\hline C. tropicalis & 42 & 92,9 & 97,8 & - \\
\hline C. kefyr & 11 & 18,2 & 90,5 & $2(4,8)$ \\
\hline C. krusei & 8 & 87,5 & 36,4 & $7(63,6)$ \\
\hline
\end{tabular}




\subsubsection{Zusammenfassung der Korrelation beider Testmethoden}

Die Korrelation der Testmethoden für Fluconazol wurde für alle 567 Isolate ermittelt. Die Übereinstimmung hinsichtlich der Konzentrationsstufen von 91,4\% sowie der Kategorien von 85,2\% können als zufriedenstellend eingeordnet werden. Als problematisch erwiesen sich die C. glabrata-Isolate, die im Etest durch hohe Anteile dosisabhängig empfindlicher oder resistenter Stämme auffielen (vgl. Abschnitt 4.3.2). Dies stellt eine mögliche Erklärung dafür dar, dass ein deutlicher Unterschied zwischen der Übereinstimmung der Testmethoden bei den empfindlichen und den resistenten Isolaten vorzuliegen scheint. Die Korrelation der Ergebnisse für Itraconazol sowohl hinsichtlich der Konzentrationsstufen als auch der Kategorie war mit 55,5\% bzw. 63,3\% niedriger als für alle anderen Antimykotika. Auch hier stach C. glabrata als Problemkeim hervor. Die Korrelation der Testmethoden für Voriconazol lag in einem guten Bereich (für Kategorien bei $90 \%$ und für Konzentrationsstufen $86,2 \%$ ). C. parapsilosis hob sich durch vollständige Übereinstimmung ab. Für Caspofungin war die Korrelation der MHK-Ergebnisse für C. albicans am schlechtesten (75,5\%), insgesamt aber zufriedenstellend (87,4\%). In Bezug auf die Kategorien war die Übereinstimmung mit 97,5\% deutlich besser. Für Amphotericin B war hinsichtlich der Kategorien eine Übereinstimmung in allen Fällen zu verzeichnen. Den Angaben für 5-Fluorcytosin lagen Testungen an allen 567 Isolaten zugrunde. Die Übereinstimmung der Konzentrationsstufen von 94,9\% sowie der Kategorien von $93,8 \%$ kann als gut bezeichnet werden. Bei allen Antimykotika fiel auf, dass bei Abweichungen der Ergebnisse der Etest häufiger höhere Konzentrationsbereiche (d.h. geringere Empfindlichkeit) angab, als die Mikrodilution.

Die Ergebnisse der Korrelation für die sechs Antimykotika im Vergleich werden in der nachstehenden Tabelle 4-26 für die getesteten Isolate gezeigt. 
Tabelle 4-26: Prozentuale Korrelation der beiden Testmethoden bezüglich der Konzentrationsstufen (MHK-Stufen) und der Bewertungskategorie sowie der Anteil der diskrepanten Ergebnisse mit höherem Etest-Ergebnis $(E>M)$ gegenüber den sechs Antimykotika, angegeben für alle Isolate (Fluconazol und 5-Fluorcytosin) bzw. alle Isolate der häufigsten Spezies, die dem Etest unterzogen wurden (andere Antimykotika)

\begin{tabular}{|c|c|c|c|c|c|c|}
\hline \multirow[b]{2}{*}{ Antimykotikum } & \multirow[b]{2}{*}{$\mathbf{N}$} & \multicolumn{2}{|c|}{ Übereinstimmung in \% } & \multirow{2}{*}{$\begin{array}{c}\text { Anzahl } \\
\begin{array}{c}(\%) \\
\text { E>M }\end{array}\end{array}$} & \multirow{2}{*}{\multicolumn{2}{|c|}{$\begin{array}{l}\text { Anzahl (\%) } \\
\text { resistenter } \\
\text { Isolate }\end{array}$}} \\
\hline & & Stufen & Kategorie & & & \\
\hline \multirow[b]{2}{*}{ Fluconazol } & \multirow[b]{2}{*}{567} & \multirow[b]{2}{*}{91,4} & \multirow[b]{2}{*}{85,2} & \multirow[b]{2}{*}{$32(5,6)$} & $\mathrm{M}$ & $20(3,6)$ \\
\hline & & & & & $\mathrm{E}$ & $32(5,7)$ \\
\hline \multirow[b]{2}{*}{ Itraconazol } & \multirow[b]{2}{*}{319} & \multirow[b]{2}{*}{55,5} & \multirow[b]{2}{*}{63,3} & \multirow[b]{2}{*}{$83(26)$} & $M$ & $101(17,8)$ \\
\hline & & & & & $\mathrm{E}$ & $118(37)$ \\
\hline \multirow[b]{2}{*}{ Voriconazol } & \multirow[b]{2}{*}{319} & \multirow[b]{2}{*}{86,2} & \multirow[b]{2}{*}{90} & \multirow[b]{2}{*}{$16(5)$} & $\mathrm{M}$ & $19(3,3)$ \\
\hline & & & & & $\mathrm{E}$ & $11(3,4)$ \\
\hline \multirow[b]{2}{*}{ Caspofungin } & \multirow[b]{2}{*}{318} & \multirow[b]{2}{*}{87,4} & \multirow[b]{2}{*}{97,5} & \multirow[b]{2}{*}{$33(10,4)$} & $M$ & $13(2,3)$ \\
\hline & & & & & E & $6(1,9)$ \\
\hline \multirow[b]{2}{*}{ Amphotericin B } & \multirow[b]{2}{*}{318} & \multirow[b]{2}{*}{-} & \multirow[b]{2}{*}{100} & \multirow[b]{2}{*}{-} & $\mathrm{M}$ & - \\
\hline & & & & & $E$ & - \\
\hline \multirow[b]{2}{*}{ 5-Fluorcytosin } & \multirow[b]{2}{*}{567} & \multirow[b]{2}{*}{93,8} & \multirow[b]{2}{*}{94,9} & \multirow[b]{2}{*}{$30(5,3)$} & $\mathrm{M}$ & $26(4,6)$ \\
\hline & & & & & $E$ & $46(8,1)$ \\
\hline
\end{tabular}




\section{$5 \quad$ Diskussion}

In der vorliegenden Arbeit wurden vor dem Hintergrund der steigenden Inzidenz über die letzten 20 Jahre (Pfaller und Diekema 2007) und der hohen Mortalität systemischer Pilzinfektionen (Garbino et al. 2002, Edmond et al. 1999, Wey et al. 1988) Epidemiologie und Resistenzlage anhand einer repräsentativen Stichprobe von Hefepilzen aus dem gesamten Bundesgebiet untersucht. Teile der Ergebnisse unserer Untersuchungen publizierten wir 2007 (Borg-von Zepelin et al.).

Bis dahin gab es keine bundesweite Übersicht über die Epidemiologie und Resistenzlage systemischer Mykosen in Deutschland. Vorherige Studien aus Deutschland blieben regional, zeitlich eng begrenzt oder hatten Stichproben kleineren Umfangs (Borg-von Zepelin et al. 1993, Büchner et al. 2002, Glasmacher et al. 1998, Knoke et al. 1997, Rüchel et al. 2002). Im Rahmen der weltweiten SENTRY-Studie (Pfaller et al. 2001) wurden zu einem geringen Anteil auch deutsche Daten ausgewertet (zwei deutsche Kliniken unter 74 teilnehmenden Instituten). Eine Studie der European Confederation of Medical Mycology (ECMM) erhob erstmals großflächig Daten aus den Jahren 1997 bis 1999 aus sieben verschiedenen europäischen Ländern, darunter Deutschland (Tortorano et al. 2004); hier waren unter 106 teilnehmenden Institutionen 13 deutsche Kliniken, so dass die Studie mehrere Regionen Deutschlands abbildete. In ihrer Dissertation untersuchte Kumm (2008) die deutschen und österreichischen Isolate der ECMM-Studie erneut hinsichtlich Resistenzen. Im Rahmen der bisher größten ARTEMIS (Advanced Research Testbed for Medical Informatics) -Studie wurden weltweit über 250.000 Candida-Isolate über den Zeitraum von 10,5 Jahren (1997 bis 2007) gesammelt (Pfaller et al. 2010). Auch hier war nur ein kleiner Anteil deutscher Isolate vertreten. In den genannten Studien wurden große geographische Unterschiede sowohl in der Speziesverteilung als auch hinsichtlich der Resistenzlage deutlich.

Seit 2004 sammelt das Nationale Referenzzentrum für Systemische Mykosen mit Hilfe des bundesweiten Labornetzwerks MykolabNet-D Isolate aus Deutschland, die seitdem regelmäßig und systematisch hinsichtlich Epidemiologie und 
Resistenzlage untersucht werden. Die von Juli 2004 bis August 2005 eingesandten Isolate sind wie beschrieben Grundlage für die vorliegende Arbeit gewesen.

Besonders gefährdet für systemische Pilzinfektionen sind immunsupprimierte und/oder hospitalisierte Patienten, die sich auf dem Boden einer schwerwiegenden Grunderkrankung invasiven Prozeduren oder Therapien unterziehen müssen. Breitspektrum-Antibiose, Kortikoidtherapie, das Vorhandensein zentraler Venenkatheter, parenterale Ernährung oder große chirurgische, insbesondere abdominale, Eingriffe sowie intensivmedizinische Betreuung stellen wichtige Risikofaktoren für nosokomiale Infektionen dar (Geffers et al. 2004, Odds et al. 2007). 42\% der von uns untersuchten Isolate stammten von intensivpflichtigen Patienten. Ähnliche Anteile publizierten Garbino et al. (2002) und Kibbler et al. (2003) mit 38-45,5\%. Die uns vorliegenden Angaben zu den Daten für Patienten auf internistischen und chirurgischen Stationen (16\% und 7,6\%) zeigen Diskrepanzen zu Daten der anderen Autoren (Garbino et al. 25\% und 21\%) und sind sicherlich begrenzt aussagekräftig, da bei über $30 \%$ unserer Isolate diese klinischen Angaben fehlten.

Eindeutig erkranken Männer häufiger an systemischen Mykosen als Frauen. Der Anteil männlicher Patienten lag unseren Ergebnissen nach bei 54,2\%. Dies entsprach Angaben zahlreicher Publikationen weltweit. Hajjeh et al. (2004) publizierten einen Anteil von 54\% für die USA, McMullan et al. (2002) 52\% für Irland. Einen größeren Anteil männlicher Patienten beobachteten andere Studien aus Dänemark (56\%), den USA (59\%), der Schweiz (67,3\%), Finnland (59-60\%) und Frankreich (68\%, Arendrup et al. 2005, Diekema et al. 2002, Garbino et al. 2002, Lyytikäinen et al. 2002, Poikonen et al. 2003 und Richet et al. 2002).

Die Inzidenz der systemischen Candidämie steigt mit zunehmendem Alter an. Diesem Muster folgend, stammten über zwei Drittel unserer Proben von Patienten, die älter als 55 Jahre alt waren (durchschnittliches Alter 60,6 Jahre). Dieses Ergebnis wurde bereits vielfach reproduziert. Hajjeh et al. (2004) gaben an, dass $72 \%$ der Patienten über 45 Jahre bzw. 45\% über 65 Jahre alt waren. Diekema et al. (2002) berichteten von einem 67\%igen Anteil über 50jähriger Patienten. Der C. glabrata-Anteil war in diesem Patientenkollektiv mit 51,2\% 
besonders hoch. Die Arbeitsgruppe beschrieb einen kontinuierlichen Anstieg von C. glabrata mit zunehmendem Alter. Der Anteil lag, unseren Daten nach, bei den über 65-Jährigen mit 9\% deutlich darunter. Die C. glabrata-Zunahme mit steigendem Alter wurde jedoch ebenfalls deutlich (Abbildung 4-3). In der Gruppe der ältesten Patienten, überwogen die Proben der Patientinnen (Tabelle 7-1, S. 95). Eine mögliche Erklärung hierfür ist die längere Lebenserwartung von Frauen.

Eine weitere Risikogruppe für die systemische Candidämie sind die Neugeborenen. Bei unreifen Frühgeborenen sind invasive Candidainfektionen die dritthäufigste Ursache von Infektionen (Chapman 2007, Laupland et al. 2005). Roilides et al. (2004) beschrieben C. albicans (65,5\%), C. parapsilosis (15,5\%) sowie C. tropicalis $(7 \%)$ als die drei Haupterreger dieser Altersgruppe. Unsere Ergebnisse zeigten mit einem Anteil von knapp 36\% eine klare Dominanz von C. parapsilosis in der Gruppe der Säuglinge und Kleinkinder; C. albicans folgt mit 29\% (ähnliche Daten von Odds. et al. 2007). Auch andere Veröffentlichungen hoben C. parapsilosis als wichtigstes Pathogen unter den Non-albicans-Erregern für diese Altersgruppe hervor (Levy et al. 1998, Kao et al. 1999, Rangel-Frausto 1999, Sandven 2000).

Weltweit wurden bisher über 30 verschiedene humanpathogene Candidaspezies isoliert (Pfaller et al. 2010). Für etwa 92\% der systemischen Candidamykosen sind die fünf Spezies C. albicans, C. glabrata, C. parapsilosis, C. tropicalis und $C$. krusei verantwortlich (Pfaller et al. 2010).

C. albicans ist weltweit die am häufigsten vorkommende Candida-Spezies. Der Anteil variiert jedoch stark nach Land und Region. Unsere Auswertungen ergaben einen C. albicans-Anteil von 56,8\%. Dies entspricht dem von Tortorano et al. (2004) im Rahmen der ECCM-Studie publizierten westeuropäischen Durchschnitt $(56,4 \%)$. Ähnlich waren die Angaben aus der SENTRY-Studie für die USA (56,2\%, Pfaller et al. 2001). Die höchsten Anteile gaben die Autoren für Italien (88\%), die Schweiz (80\%) und Frankreich (73\%) an, während Deutschland mit 21\% neben Brasilien (24\%) und Spanien (29\%) den niedrigsten C. albicansAnteil der gesamten Studie aufwies. Trotz der großen geographischen Schwankungen ist $C$. albicans unbestritten die weltweit dominierende CandidaSpezies (Pfaller et al. 2010, Tortorano et al. 2006). Dies wurde in einer Vielzahl 
europäischer sowie außereuropäischer Studien reproduziert (Ásmundsdóttir et al. 2002, Boo et al. 2005, Chakrabarti et al. 1992, Chen et al. 1997, Kibbler et al. 2003, Klingspor et al. 2004, Luzzati et al. 2000, Odds et al. 2007, Poikonen et al. 2003, Yapar et al. 2006).

Die zweithäufigste Spezies ist in der von uns untersuchten Stichprobe C. glabrata $(18,5 \%)$. Dazu kongruent sind die us-amerikanischen Ergebnisse von Pfaller et al. (18\%, 1999). Der westeuropäische Durchschnitt wurde in der ECCM-Studie mit $13,6 \%$ beziffert (Tortorano et al. 2004). Die Häufigkeit von C. glabrata betrug anderen Arbeiten zufolge $12 \%$ in Island, Frankreich und Norwegen, 15\% in Schweden und der Schweiz sowie 21\% in Schottland (Ásmundsdóttir et al. 2002, Klingspor et al. 2004, Marchetti et al. 2004, Martin et al. 2005, Odds et al. 2007, Sandven et al. 1998).

Viel diskutiert wurde die Zunahme der Non-albicans-Isolate im Verhältnis zu den C. albicans-Isolaten und mögliche Gründe hierfür. Während die Arbeitsgruppen von Garbino (2002) und Marchetti (2004) in der Schweiz über den Beobachtungszeitraum von 10 Jahren keinen Speziesshift feststellten, gab es zahlreiche Publikationen, die dies für andere Länder belegten. Die ARTEMISStudie wies weltweit eine Abnahme der C. albicans-Stämme um 5\% über den Studienzeitraum (1997-2007) sowie eine zeitgleiche Zunahme von C. glabrata um 1,5\% und von C. tropicalis um 2,6\% nach (Pfaller et al. 2010). Unsere Daten zeigten eine ähnliche Entwicklung für das Klinikum der Georg-August-Universität Göttingen. Rüchel et al. gaben 2002 einen C. albicans-Anteil von 80,2\% für das Klinikum an, während der von uns erhobene Anteil für die Jahre 2004/2005 bei $69,7 \%$ (nur Göttingen) lag, so dass sich ein Unterschied von 10,5\% ergab. Zahlreiche andere Publikationen beobachteten den gleichen Trend (Diekema et al. 2002, Richet et al. 2002, Trick et al. 2002, Viscoli et al. 1999). Parallel zum C. albicans-Rückgang, stieg C. glabrata an. Die ECCM-Studie berichtete von einem C. glabrata-Anteil von 13,6\% unter den deutschen Isolaten aus den Jahren 1997 bis 1999 (Tortorano et al. 2004). Unsere Ergebnisse lagen um 5\% höher und zeigten somit einen klaren Trend. Auch mehrere Autoren aus den USA (Abi-Said et al. 1997) sowie aus Europa (Coleman et al. 1998, Sandven 2000) beschrieben einen deutlichen C. glabrata-Zuwachs. Für diese Zunahme wurde vor allem der 
breite Einsatz von Azolen, insbesondere zur Prophylaxe bei immunsupprimierten Patienten, verantwortlich gemacht (Almirante et al. 2005). Eine Infektion mit einem Non-albicans-Isolat ist wahrscheinlicher, wenn eine vorherige antimykotische Prophylaxe durchgeführt wurde (Nguyen et al. 1996). Die Mechanismen hierfür sind vielfältig. Eine Erklärung für die Resistenzentwicklung von C. glabrata unter Azolexposition ist beispielsweise die Hochregulation von Efflux-Pumpen, die zum verstärkten Ausschleusen des Antimykotikums aus der Zelle führt (Sanglard und Odds 2002).

C. parapsilosis war mit einem Anteil von 8,1\% unter den Candida-Isolaten die dritthäufigste Spezies. Veröffentlichungen aus den USA und aus Europa berichteten von ähnlichen Verteilungen (Kibbler et al. 2003, Nguyen et al. 1996, Pfaller et al. 1999). Die im Rahmen der ECCM-Studie ermittelten durchschnittlichen westeuropäischen Anteile lagen für C. parapsilosis mit 13,3\% etwas höher (Tortorano et al. 2004). Deutlich höhere Anteile wurden in mehreren einzelnen europäischen Studien beschrieben, so aus Spanien und Italien (jeweils 23\%, Almirante et al. 2005, Cuenca-Estrella et al. 2005, Luzzati et al. 2000), Dänemark (20\%, Arendrup et al. 2005) sowie aus der Slowakei (20-40\%, Krcmery und Barnes 2002). C. parapsilosis gilt als wichtigster Erreger von Candidämien bei Säuglingen (Levy et al. 1998, Rangel-Frausto 1999, Roilides et al. 2004, Sandven 2000). Eine mögliche Erklärung des geringeren C. parapsilosis-Anteils bei unseren Daten könnte der insgesamt niedrige Anteil an Proben von Säuglingen und Kleinkindern sein.

In der vorliegenden Studie rangierte die Spezies $C$. tropicalis mit 7,4\% auf dem vierten Platz. Die ECCM-Studie ergab für Westeuropa einen ähnlichen $C$. tropicalis-Anteil von 7,2\% (Tortorano et al. 2004). Davon abweichende Berichte über $C$. tropicalis als zweithäufigste Spezies stammen sowohl aus Lateinamerika, als auch aus China und der Türkei (Chen et al. 1997, Colombo et al. 1999, Godoy et al. 2003, Yapar et al. 2006).

C. kefyr belegt mit einem Anteil von 1,9\% den fünften Platz. Die Angaben anderer Publikationen reichen hierzu passend von 0,1-0,6\% (Hajjeh et al. 2004, Pfaller et al. 2006b, Tortorano et al. 2004) bis zu 2,2\% (Eloy et al. 2006).

C. krusei rangiert auf dem sechsten Platz bei einem Anteil von 1,4\%. Dies deckt 
sich mit Angaben von Vorkommen zwischen 1\% und 2\% der beiden großen ECCM- und SENTRY-Studien sowie auch zahlreicher weiterer Veröffentlichungen (Fleck et al. 2007, Hajjeh et al. 2004, Marchetti et al. 2004, Pfaller et al. 1999, Pfaller et al. 2001).

Mit dem vermehrten Auftreten von Resistenzen und der Einführung neuerer Antimykotika-Gruppen hat die Empfindlichkeitstestung bei Pilzisolaten in den letzten Jahren zunehmend an Bedeutung gewonnen. Goldstandard und Referenzmethode der Resistenztestung von Candida ist die Mikrodilution. Bereits 1982 begann das "National Committee for Clinical Laboratory Standards“ (NCCLS), später umbenannt in "Clinical and Laboratory Standards Institute" (CLSI) mit der Entwicklung von standardisierten Testmethoden zur reproduzierbaren Resistenzbestimmung von Pilzen, die insbesondere zuverlässige internationale Vergleiche von Ergebnissen ermöglichen sollte (Arikan 2007, Rex et al. 1997). 1992 erschien erstmalig eine detaillierte Arbeitsanleitung für die Testung von Hefen, die in den Jahren 1997 und 2002 überarbeitet wurde und auf der die vorliegende Arbeit basiert (NCCLS M27-A2 2002, Sanglard und Odds 2002). Diese Version wurde seit Fertigstellung der Laborarbeiten für diese Arbeit erneut aktualisiert und 2008 erstmals mit Breakpoints für Echinocandine publiziert (CLSI M27-A3 2008).

Mit dem Etest wurde ein kommerziell erwerblicher Test eingeführt, der im Vergleich zur Mikrodilution deutlich einfacher in der Handhabe ist, so dass Arbeits-, Zeit- und damit auch Personalaufwand geringer sind. Zahlreiche Untersuchungen wiesen eine zufriedenstellende Übereinstimmung zu den Ergebnissen im Vergleich zum Goldstandard nach, so dass der Etest bereits lange Einzug in den klinischen Alltag gefunden hat und als Alternativmethode weitestgehend akzeptiert ist (Alexander et al. 2007, Arikan 2007, Colombo et al. 1995, Espinel-Ingroff et al. 1996, Pfaller et al. 1996).

Die Azole wurden bereits Ende der 60iger Jahre entdeckt. Wirkmechanismus dieser Gruppe ist die Hemmung der Ergosterolsynthese durch Blockade Cytochrom-P450-abhängiger Enzyme in der Zellwand des Pilzes, so dass eine fungistatische, in hoher Dosis auch fungizide, Wirkung erzielt wird (Georgeopapadakou und Walsh 1996). Das Wirkspektrum ist breit und erfasst 
nahezu alle Pilzspezies. Fluconazol ist seit 1990 im klinischen Einsatz; Itraconazol und Voriconazol sind Weiterentwicklungen des Fluconazol (Pfaller et al. 2006c, Rex et al. 2000).

Bei über $90 \%$ aller durch Mikrodilution getesteten Isolate lag eine gute Wirksamkeit gegenüber Fluconazol vor. Die herabgesetzte Empfindlichkeit lag vor allem bei den Spezies C. glabrata (20\%) und C. krusei $(87,5 \%)$ vor. Bei beiden Spezies ist eine intrinsische Resistenz gegenüber Azolen bekannt (Eggimann et al. 2003, Lamping et al. 2009, Martin et al. 2005). Im Rahmen der us-amerikanischen EIEIO (Emerging Infections and the Epidemiology of IOWA Organisms)-Studie beschrieben Diekema et al. (2002) einen ähnlich hohen Anteil C. glabrata-Isolate mit herabgesetzter Empfindlichkeit (10\% resistent und 10\% dosisabhängig sensibel). Bei der SENTRY-Studie wurden FluconazolResistenzen im Bereich von $17 \%$ bis $52 \%$ ermittelt (Pfaller et al. 2001). C. krusei war durchgehend resistent (ebenda). Zahlreiche andere Autoren beschrieben dieselbe Problematik (Fleck et al. 2007, Kibbler et al. 2003, Krcmery und Barnes 2002). Für die C. tropicalis-Isolate lag nach unseren Daten eine FluconazolResistenz von knapp $5 \%$ vor. Dies stimmte überein mit mehreren Studien aus den USA (Hajjeh et al. 2004, Pfaller und Diekema 2007). Die Spezies C. inconspicua und $C$. norvegensis weisen ebenfalls eine intrinsische Resistenz gegenüber Fluconazol auf (Tortorano et al. 2003). Analog dazu stellten auch wir eine herabgesetzte Sensibilität fest. Die Publikationen von Pfaller und Diekema (2007) sowie Sandven et al. (1997) bestätigten diese Beobachtungen. Trotz der mangelhaften Wirksamkeit bei $C$. glabrata und $C$. krusei sowie den klinisch weniger häufig relevanten $C$. inconspicua und $C$. norvegensis liegt für den größten Teil der Candida spp. noch immer eine gute Wirksamkeit von Fluconazol vor.

Bei der Testung mit dem Etest war der Anteil der sensiblen Isolate mit $82 \%$ geringer. Dabei stach auch bei dieser Methode C. krusei durch eine 100\%ige Resistenz hervor. Die C. glabrata-Isolate waren zu über zwei Dritteln in ihrer Empfindlichkeit herabgesetzt. Die intrinsische Resistenz der genannten Spezies kommt somit durch den Etest genauso zum Tragen wie bei der Mikrodilution. Diese Beobachtungen teilten zahlreiche Autoren (Chryssanthou 2001, Guinea et 
al. 2006, Lamping et al. 2009).

Als eine der ersten Forschungsgruppen untersuchten Sewell et al. (1994) die Korrelation zwischen dem damals neu entwickelten Etest und der Mikrodilution anhand von Fluconazol. Seither folgten viele Studien, die die Methoden miteinander verglichen (Maxwell et al. 2003, Posteraro et al. 2000, Warnock et al. 1998). Unser Ergebnis $(91,4 \%)$ lag im Bereich der von den anderen Autoren veröffentlichten Daten $(79,4 \%$ - 96\%, ebenda) und ist somit für den klinischen Alltag als akzeptabel einzustufen. Als problematisch eingestuft wurde die Korrelation bei C. glabrata und C. krusei, da das Ablesen des Etests durch Wachstum einzelner Kolonien in die Inhibitionszone erschwert wurde (Koç et al. 2000, Schmalreck et al. 1996). Eine von Morace et al. (2002) publizierte Studie ergab eine Korrelation für C. glabrata und C. krusei von $68,4 \%$ bzw. $58,3 \%$, wobei unsere Ergebnisse hinsichtlich der Konzentrationsstufen mit 86,7\% und $100 \%$ in einem deutlich besseren Bereich liegen. Alexander et al. (2007) berichteten sogar von nur 40\%iger Übereinstimmung bei $C$. krusei. Matar et al. (2003) beschrieben eine höhere Übereinstimmung bei den sensiblen Isolaten als bei den resistenten Isolaten. Dies entspricht unseren Ergebnissen. Die Korrelation lag bei den in der Referenzmethode sensiblen Stämmen bei 94,1\%; bei den in der Referenzmethode als resistent getesteten Stämmen bei nur 80,9\%. Des Weiteren beschrieben Pfaller et al. (2003a) eine schlechtere Übereinstimmung bezüglich der Kategorien als bezüglich der Konzentrationsstufen. Sie erklärten dies durch kleinere Abweichungen der MHKs im oberen Empfindlichkeitsbereich, durch die viele Isolate als „dosisabhängig sensibel“ anstatt "sensibel“ kategorisiert wurden. Dieses Phänomen trat auch bei unseren Testungen auf.

Mit der Einführung der Arbeitsanleitung für die Mikrodilutionstestung wurden durch NCCLS auch Breakpoints zur Bewertung der MHKs veröffentlicht. Die zugrunde liegenden Daten für Fluconazol basierten zu etwa $80 \%$ auf Studien zu Schleimhaut-Candidosen und beinhalteten Isolate mit überwiegend niedrigen MHKs (Rex et al. 1997). Da die Hefen im klinischen Altag häufig aus Blutkulturen stammen und beispielsweise nach Fluconazol-Prophylaxe höhere MHKs aufweisen, wurde hinterfragt, ob die Bewertungskriterien auf dieses Spektrum 
von Hefen genauso anwendbar wären. Um dies zu untersuchen, werteten Pfaller et al. (2006c) zusätzlich mehrere Studien invasiver Candidosen aus und konnten die vorgeschlagenen Breakpoints auch für diese Hefen bestätigen - eine Korrelation zum klinischen Outcome konnte nachgewiesen werden. Zusammenfassend wird Fluconazol sicherlich auch in naher Zukunft weiterhin die Rolle des in Deutschland am häufigsten eingesetzten Antimykotikums beibehalten. Es sollte allerdings immer die Differenzierung der Isolate angestrebt werden, so dass eine antimykotische Therapie durchgeführt werden kann, die die Resistenzlage bestimmter Spezies miteinbezieht (Arikan 2007, Oxman et al. 2010).

Itraconazol wies im Vergleich zu den anderen Antimykotika die niedrigste Wirksamkeit auf $(63 \%$ sensible, $19,2 \%$ intermediäre und $17,8 \%$ resistente Isolate). Dazu trug die ungenügende Wirksamkeit der Triazole gegenüber $C$. glabrata und C. krusei stark bei. Unsere C. glabrata-Isolate wiesen eine Resistenzrate von 59,1\% auf. Die $\mathrm{MHK}_{90}$ lag für alle Isolate bei $1 \mu \mathrm{g} / \mathrm{ml}$; Glasmacher et al. (1999) gaben die durchschnittlich in vivo erzielten niedrigsten Serumspiegel mit $0,5-0,67 \mu \mathrm{g} / \mathrm{ml}$ bei systemischer Therapie an. Diese wäre für eine effektive antimykotische Therapie ausreichend. Hohe Resistenzraten fanden sich auch nach neueren Daten von Pfaller et al. (39\%, 2011b), von Metin et al. $(54,5 \%, 2011)$ sowie nach Odds et al. (herabgesetzte Empfindlichkeit bei über $70 \%, 2007)$. Andere Untersuchungen von Diekema et al. (2002) ergaben einen Resistenzanteil von 53\%. Für C. krusei publizierten Pfaller et al. (1998b) Resistenzen von über $66 \%$. Unsere Ergebnisse lagen mit $87,5 \%$ jedoch noch höher. Angesichts dieser Ergebnisse sollten vor allem Daten zur in vivoWirksamkeit und dem klinischem Outcome berücksichtigt und weiterhin gesammelt werden (Glasmacher et al. 1999 und 1998). Zufriedenstellende Wirksamkeit war somit nur bei $C$. albicans festzustellen $(87,9 \%)$, wobei der Etest eine Wirksamkeit in $92,8 \%$ detektierte. Des Weiteren zeigten die EtestErgebnisse für Itraconazol passend $z u$ den beschriebenen Daten für die Mikrodilution ebenfalls erhebliche Resistenzen bei den Problemkeimen $C$. glabrata (96\% herabgesetzte Empfindlichkeit) und C. krusei $(71 \%$ herabgesetzte Empfindlichkeit). In der Arbeit von Metin et al. (2011) wird die durch den Etest detektierte Resistenzrate bei C. glabrata mit $75 \%$ angegeben. Empfindlichkeit 
konnte mit dem Etest nur für 55,2\% nachgewiesen werden.

Die Korrelation der Testmethoden war für Itraconazol insgesamt mäßig (55,5\% +/- zwei Konzentrationsstufen, 63,3\% kategorial). Von ähnlich geringen Übereinstimmungen berichteten Negri et al. (2009, 64\% abhängig von den Konzentrationsstufen) und Chryssanthou (abhängig von Konzentrationsstufen $72 \%$ für C. albicans bzw. $60 \%$ für die Non-albicans-Spezies, 2001). Auf alle Spezies bezogen publizierten Alexander et al. (2007) mit $80 \%$ kategorialer und 95\% Übereinstimmung hinsichtlich der MHK-Stufen deutlich bessere Ergebnisse. Doch auch bei dieser Arbeitsgruppe stachen C. glabrata, C. krusei und auch $C$. tropicalis aufgrund der deutlich schlechteren Übereinstimmungen im Vergleich zu den anderen Spezies hervor. Vor allem die kategoriale Übereinstimmung war mit $0 \%$ für C. krusei und $62 \%$ für C. tropicalis sehr gering (unsere Ergebnisse $43 \%$ und 57\%). Bei der Mehrzahl der abweichenden Konzentrationsstufen war das Etest-Ergebnis höher als das der Mikrodilution; dies ist anhand unserer Ergebnisse nicht nachzuvollziehen (in nur 26\% höheres Etest- als MikrodilutionsErgebnis). Da die Empfindlichkeit bei beiden Methoden so niedrig im Vergleich zu den anderen Antimykotika war, sollten die klinische Anwendung von Itraconazol bei den gegebenen Breakpoints, sowie die Testung per Etest bei nicht ausreichender Korrelation zur Mikrodilution kritisch gesehen werden.

Voriconazol gehört zur neueren Generation der Azole und gilt als eines der effektivsten in dieser Gruppe (Hoban et al. 1999, Kibbler et al. 2003). Im Vergleich mit den anderen Azolen war Voriconazol auch bei unseren Testungen das potenteste Antimykotikum. Während die anderen Azole hohe Resistenzraten bei C. glabrata und C. krusei aufwiesen, hemmte Voriconazol $87 \%$ bzw. $86 \%$ effektiv in ihrem Wachstum. Unseren ähnliche Resistenzraten beschrieben Odds et al. (2007) in einer regionalen Studie für Schottland. Dies wird erklärt durch die höhere Bindungsfähigkeit des Voriconazols an das Cytochom P450 im Vergleich zu Fluconazol (Pfaller und Diekema 2007). Problematisch waren in der Testung C. tropicalis-Isolate (14,3\% resistent). Neuere Daten aus einer regionalen Studie aus Spanien kommen zu einem ähnlich hohen Ergebnis (17,6\%, Guinea et al. 2010). Die herabgesetzte Empfindlichkeit dieser Isolate könnte auf dem „trailing growth"-Phänomen basieren, durch das einige Isolate fälschlicherweise als 
resistent eingestuft werden können (vgl. Abschnitt 3.4.7.1).

Die mit dem Etest untersuchte Empfindlichkeit gegenüber Voriconazol lag bei 98$100 \%$, wobei die C. glabrata-Isolate mit $79,6 \%$ geringere Sensibilität aufwiesen. Insgesamt wurde überwiegend von einer anhaltend guten Wirksamkeit von Voriconazol insbesondere bei C. glabrata berichtet (Fleck et al. 2007, Metin et al. 2011).

Die Korrelation der beiden Testmethoden ergab für Voriconazol eine Übereinstimmung der Konzentrationsstufen von 86,2\%. Mehrere Studien erzielten mit 87 bis $98 \%$ höhere Übereinstimmungen (Fleck et al. 2007, Matar et al. 2003, Pfaller et al. 2000). Matar et al. (2003) prüften die Korrelation für die durch die Referenzmethode sensibel und resistent getesteten Stämme separat. Die resistenten Isolate wiesen dabei eine deutlich schlechtere Übereinstimmung $(\leq 83 \%)$. auf. Dieses Phänomen ist auch anhand unserer Ergebnisse nachzuvollziehen.

Da die verschiedenen Azole alle an dem oben beschriebenen Wirkprinzip ansetzen, können sich zwischen den Azolen Kreuzresistenzen ausbilden. Unter der Gruppe der Isolate, die eine herabgesetzte Empfindlichkeit gegenüber Fluconazol aufwiesen, wurde C. glabrata separat betrachtet, um bei dem Problemkeim die Wirksamkeit der anderen Antimykotika zu beurteilen. Itraconazol war dabei keine Alternative, Voriconazol dennoch in über der Hälfte der Fälle gut wirksam. Ähnliches berichteten Diekema et al. (2002). Tortorano et al. (2003) schilderten, dass Kreuzresistenzen zu den neueren Azolen selten sind, so dass die Anwendung von Voriconazol meist möglich ist. Cuenca-Estrella et al. (2005) veröffentlichten Daten, die entgegengesetzt dazu eine herabgesetzte Wirksamkeit von Voriconazol beschrieben, wenn bereits Fluconazol und Itraconazol nicht mehr wirksam waren. Das Auftreten der Kreuzresistenzen verdeutlicht die Notwendigkeit des sorgfältigen Umgangs mit den Azolen, um weitere Resistenzbildungen nicht zu fördern (Pfaller et al. 2001). Nach anhaltender oraler Fluconazol-Prophylaxe bei AIDS-Patienten verdoppelte sich die MHK gegenüber Fluconazol der von diesen Patienten stammenden CandidaIsolate im Durchschnitt alle 31 Tage (Bennett et al. 2004). Pfaller et al. (2006a) beschrieben bereits einen deutlichen Unterschied des Auftretens der 
Kreuzresistenzen bei den unterschiedlichen Spezies und hoben aufgrund der oben beschriebenen intrinsischen Resistenz gegenüber Azolen C. glabrata und C. krusei als problematisch hervor.

Besonderes Interesse verdient Caspofungin, das als erstes Echinocandin seit 2002 für den klinischen Einsatz zugelassen wurde. Es wirkt über eine Synthesehemmung des $(1,3)$ ß-D-Glukans, dem Hauptbestandteil der Pilzzelle, konzentrationsabhängig fungizid auf Candida und Aspergillus (Douglas et al. 1997, Odds et al. 2004). Da (1,3)ß-D-Glukan in der Zellwand des Menschen nicht vorkommt, ist das Nebenwirkungsprofil günstig. Nachteilig sind unzureichende Wirkspiegel in ZNS und Urin.

Erst 2008 veröffentlichte das CLSI „breakpoints“ für Caspofungin, wobei festgelegt wurde, welche Isolate sensibel wären und welche nicht mehr in den sensiblen Bereich fielen, ohne Letztere als resistent zu bezeichnen, da zu jenem Zeitpunkt keine Resistenzen gegenüber Caspofungin bekannt waren (Pfaller et al. 2008a). Es wurden somit die Kategorien "sensibel“ und „nicht-sensibel“ unterschieden und die Empfehlung ausgesprochen, klinisch resistente Isolate in Referenzlabors auf Resistenzmechanismen untersuchen zu lassen. Kürzlich wurde jedoch der Tatsache Rechnung getragen, dass im Verlauf klinisch relevante Resistenzen auftraten, dabei die MHKs aber nicht die vorgeschlagene Bewertungsgrenze von $\leq 2 \mu \mathrm{g} / \mathrm{ml}$ überstiegen. CLSI publizierte neue, diesmal speziesspezifische Breakpoints. Die Arbeitsgruppe um Pfaller et al. werteten systematisch vorhandene Studien zu Molekulargenetik, Pharmakodynamik und -kinetik und klinischen Verläufen in Bezug auf Echinocandine aus und bestätigten, dass die speziesspezifischen Bewertungskriterien besser in der Lage sind, klinisch resistente Isolate zu detektieren (Pfaller et al. 2011a). So sind mit klinischer Resistenz beispielsweise Mutationen zweier die $(1,3)$ ß-DGlukansynthetase codierender Gene (fks1 und fks2) verbunden, die mit einer erhöhten MHK des Isolates einhergehen, die aber nicht unbedingt über den alten Breakpoint von $\leq 2 \mu \mathrm{g} / \mathrm{ml}$ hinausgingen und somit fälschlich für sensibel klassifiziert worden wären (ebenda). Sicherlich werden zukünftig analog zu Caspofungin auch speziesspezifische Breakpoints für die anderen Antimykotika entwickelt werden. Unsere Isolate von C. albicans, C. parapsilosis und auch die 
oft problematischen Spezies C. glabrata, C. tropicalis und C. krusei ließen sich in ihrem Wachstum durch Caspofungin effektiv hemmen (94,3-100\% Sensibilität). Lediglich ein C. glabrata-Isolat war resistent und fünf Isolate dieser Spezies waren intermediär sensibel. Eine verminderte Wirksamkeit lag bei $\mathrm{Cr}$. neoformans, C. guilliermondii, sowie Trichosporon spp. vor. Mehrere Studien fügen sich gut in unsere Beobachtungen ein (Arendrup et al. 2005, Barchiesi et al. 2006, Bartizal und Odds 2003, Cuenca-Estrella et al. 2005, Fleck et al. 2007, Pfaller und Diekema 2007, Pfaller et al. 2003b und 2008b). Die vorliegenden Ergebnisse heben die gute Wirksamkeit von Caspofungin gegenüber dem großen Spektrum von Candida spp. hervor (Pfaller et al. 2003b). Auf die oben beschriebene Gruppe der C. glabrata-Isolate mit herabgesetzter Empfindlichkeit gegenüber den Azolen bezogen, ist anzumerken, dass diese durchgehend niedrige MHKs $(\leq 0,5 \mu \mathrm{g} / \mathrm{ml})$ gegenüber Caspofungin aufwiesen. Somit stellt Caspofungin bei dieser Gruppe eine effektive Alternative dar. Hof (2008) stellte ebenfalls fest, dass Caspofungin-Resistenzen bisher selten sind und die primär oder sekundär azol-resistenten Hefen mit diesem Antimykotikum therapiert werden können.

Die für die Mikrodilution beschriebenen Ergebnisse treffen auch für den Etest zu. Von einer guten Empfindlichkeit ist auszugehen; die $\mathrm{MHK}_{90}$ liegt für alle Isolate bei $0,5 \mu \mathrm{g} / \mathrm{ml}$. Andere Arbeitsgruppen bestätigten diese Etest-Ergebnisse (Alexander et al. 2007, Fleck et al. 2007). Bisher lag nur eine Arbeit vor, die die neuesten Breakpoints für Caspofungin anwendete (Axner-Elings et al. 2011). Es wurde von guter Wirksamkeit berichtet. Nur zwei (von fünf) C. krusei-Isolate befanden sich im intermediären Wirkbereich und ein C. tropicalis-Stamm (von acht) war resistent. Bei diesem C. tropicalis-Stamm konnte per Gensequenzanalyse eine Mutation der fks-1-Region nachgewiesen werden, die die Caspofungin-Resistenz vermittelt (Garcia-Effron et al. 2008). Alle unsere $C$. krusei-Isolate waren empfindlich, ein $C$. tropicalis-lsolat war jedoch nur intermediär empfindlich.

Die Korrelation der Testmethoden hinsichtlich Konzentrationsstufen lag für Caspofungin insgesamt nur bei $87 \%$, wobei die schlechteste Übereinstimmung, anders als von anderen Autoren beschrieben, vor allem bei $C$. albicans vorlag. 
Weitere Spezies mit Übereinstimmungen zwischen 85 und $88 \%$ waren $C$. tropicalis und C. krusei. Dieses Muster geht auch aus der Arbeit von Guinea et al. (2010) hervor. Chryssanthou und Cuenca-Estrella (2002) erzielten wie wir eine Gesamtübereinstimmung von $87 \%$. Die meisten Arbeitsgruppen publizierten jedoch über 90\%ige Übereinstimmungen zwischen den Testmethoden (Alexander et al. 2007: 92\%, Fleck et al. 2007: 91\%-98\%, Laverdiere et al. 2002: 81\%-97\%). Hinsichtlich der Kategorien lag auch unsere Korrelation für alle Isolate bei $97,5 \%$.

Amphotericin B ist ein von Streptomyces produziertes Polyen, das 1956 entdeckt wurde und somit zu den ältesten Antimykotika gehört. Es entfaltet seine fungizide Wirkung dadurch, dass es sich mit dem Sterin der zytoplasmatischen Pilzzellmembran verbindet, die Membranpermeabilität erhöht und schließlich zum Zelltod führt (Georgeopapadakou und Walsh 1996). Das Spektrum ist breit und beinhaltet, ausser der Dermatophyten, alle Pilze. Aufgrund des ungünstigen Nebenwirkungsprofils, insbesondere der ausgeprägten Nephro- und Hepatotoxizität ist es aber im klinischen Alltag fast nur noch als Kombinationspräparat im Einsatz.

Alle per Mikrodilution gegenüber Amphotericin B getesteten Isolate wiesen durchgängige Sensibilität auf. Dies entspricht einer Reihe anderer Veröffentlichungen (Arendrup et al. 2005, Cuenca-Estrella et al. 2005, Fleck et al. 2007, Hajjeh et al. 2004, Marchetti et al. 2004). 92\% unserer Isolate kumulierten bei einer MHK von 0,5 und $1 \mu \mathrm{g} / \mathrm{ml}$. Eine solche Kumulation wurde auch von Diekema et al. (2002 und 2009) geschildert. Berichte über eine zunehmende Resistenzbildung von C. glabrata gegenüber Amphotericin B (Rex et al. 2000), C. krusei (Kao et al. 1999) oder das Vorliegen einer intrinsischen Resistenz bei $C$. Iusitaniae (Pfaller et al. 1994) können anhand unserer Ergebnisse nicht bestätigt werden. Auch Diekema et al. konnten in ihrer 2009 veröffentlichten Arbeit über seltenere Candida-Spezies keine primäre Resistenz von C. Iusitaniae gegenüber Amphotericin B feststellen, wiesen jedoch darauf hin, dass sich unter der Therapie nicht selten sekundäre Resistenzen entwickeln.

Auch die von uns getesteten Isolate waren gegenüber dem Amphotericin-B-Etest durchgehend sensibel $\left(\mathrm{MHK}_{90}\right.$ von $0,125 \mu \mathrm{g} / \mathrm{ml}$ ). Ähnliche Ergebnisse wurden im 
Rahmen der europäischen ECCM-Studie erhoben (durchgehende $\mathrm{MHK}_{90}$ von $\leq 0,5 \mu \mathrm{g} / \mathrm{ml}$, Tortorano et al. 2003).

Die Übereinstimmung der Ergebnisse der Testmethoden wurde aufgrund der oben beschriebenen Kumulation der Isolate nur hinsichtlich der Kategorien beurteilt und war in allen Fällen übereinstimmend. Warnock et al. (1998) beobachteten bezüglich der Korrelation der Testmethoden höhere EtestErgebnisse. Dies konnten wir aufgrund der beschriebenen Kumulation der Isolate bei $0,5 \mu \mathrm{g} / \mathrm{ml}$ und $1 \mu \mathrm{g} / \mathrm{ml}$ bei der Mikrodilution nicht nachvollziehen. Alle von uns erhobenen Etest-Ergebnisse waren deutlich niedriger (ähnlich bei Fleck et al. 2007). Mehrere Autoren sahen aufgrund der kumulierenden MHKs in der Mikrodilution keine adäquate Methode zur Detektion Amphotericin-B-resistenter Isolate (Nguyen et al. 1998, Park JY et al. 2008, Wanger et al. 1995). Auch unsere Ergebnisse zeigten eine breitere Verteilung der MHKs beim Etest $(<0,0313-1 \mu \mathrm{g} / \mathrm{ml})$ im Vergleich zur Mikrodilution $(0,0625-1 \mu \mathrm{g} / \mathrm{ml})$. Bei beiden Methoden wurden jedoch keine Resistenzen nachgewiesen.

Das Basenanalogon 5-Fluorcytosin wird statt Cytosin in die Pilzzelle eingeschleust. Dort wird es in 5-Fluoruracil umgewandelt und hemmt u.a. die Nukleinsäuresynthese, wodurch es fungistatisch wirkt. 5-Fluorcytosin wird heute nur selten in der Monotherapie eingesetzt, da sich im Verlauf der Therapie häufig sekundäre Resistenzen entwickeln (Cuenca-Estrella et al. 2001). In der Kombinationstherapie beispielsweise mit den besser verträglichen Azolen oder mit Amphotericin B ist es jedoch ein wertvolles Medikament gegenüber Candida, Cryptococcus und Aspergillus geblieben (Odds et al. 2003, Viviani 1995).

Die Empfindlichkeit der von uns getesteten Isolate gegenüber 5-Fluorcytosin ist hoch (93,7\% sensible Isolate). Ähnliche Ergebnisse (95\%) erhielten Pfaller et al. (2002) bei der Testung von über 8000 weltweit generierten Isolaten. Dies bestätigt, wie zahlreiche andere Studien, die anhaltend gute Wirksamkeit von 5Fluorcytosin (Ásmundsdóttir et al. 2002, Cuenca-Estrella et al. 2001, Hajjeh et al. 2004, Kibbler et al. 2003, Tortorano et al. 2006). Anhand unserer Daten zeigt sich allerdings eine verminderte Wirksamkeit bei $C$. tropicalis $\left(\mathrm{MHK}_{90}>16 \mu \mathrm{g} / \mathrm{ml}\right.$, $59,5 \%$ resistente Isolate). Diese wurde für andere europäische Länder nicht beschrieben und könnte eine deutsche Besonderheit sein. Fleck et al. (2007) 
veröffentlichten für die Universitätsklinik Mannheim ebenfalls eine Resistenzrate von $58,3 \%$ für $C$. tropicalis. Für die deutschen Isolate der ECMM-Isolate beschrieb Kumm (2008) eine Resistenzrate von 41,2\%, des Weiteren waren $11,7 \%$ intermediär empfindlich. Zusätzlich fällt in unseren Daten C. krusei durch herabgesetzte Empfindlichkeit (87\%) auf. Dies wurde durch die genannte Arbeit von Fleck et al. bestätigt (Resistenzrate bei $84,2 \%$ ), aber als Trend auch außerhalb Deutschlands beschrieben (Cuenca-Estrella et al. 2005, Tortorano et al. 2003). Primäre Resistenzen gegenüber 5-Fluorcytosin bei Cr. neoformans (Cuenca-Estrella et al. 2001) können anhand unserer Ergebnisse nicht nachvollzogen werden.

Mit dem Etest wurden $91 \%$ der Isolate sensibel gegenüber 5-Fluorcytosin getestet. Resistenzen traten wie bei der Mikrodilution vor allem bei $C$. tropicalis $(59,5 \%)$ und C. krusei $(87,5 \%)$ auf. Chryssanthou (2001) beschrieb für C. krusei sogar eine durchgehende Resistenz gegenüber 5-Fluorcytosin. Auffallend war, dass der Etest bei C. kefyr eine herabgesetzte Wirksamkeit von 5-Fluorcytosin nachwies (63\% herabgesetzte Empfindlichkeit), die nicht durch die Mikrodilution detektiert wurde (100\% empfindliche Stämme). Andere Autoren scheinen diese Beobachtungen nicht gemacht zu haben. Monotherapien sollten aufgrund der raschen Ausbildung von sekundären Resistenzen in jedem Fall vermieden werden.

Die von uns erzielte Korrelation der Testmethoden lag bei $94 \%$ für 5 -Fluorcytosin und war somit gut. Problematisch war die Übereinstimmung bei C. krusei. Trotz guter quantitativer Ergebnisse, lag die qualitative, d.h. kategoriale Korrelation bei nur 25\%, da durch die Mikrodilution 87,5\% der Isolate als intermediär klassifiziert wurden, durch den Etest jedoch diese $87,5 \%$ als resistent eingestuft. Alexander et al. (2007) berichteten von der gleichen Problematik und erzielten eine kategoriale Übereinstimmung von nur $20 \%$.

Diese Arbeit hat einen für Deutschland repräsentativen Überblick über die Epidemiologie von Hefen bezogen auf den Zeitraum 2004/2005 gegeben. Hinsichtlich der Resistenzlage gab es einige deutsche Besonderheiten; im wesentlichen entsprachen die Ergebnisse aber europäischen und außereuropäischen Publikationen. Bis auf Itraconazol war die Empfindlichkeit 
gegenüber den Antimykotika gut. Bei den Azolen Fluconazol und Itraconazol war die Testung für die Spezies mit bekannter primärer bzw. sekundärer Resistenz schwierig, dies galt insbesondere für den Etest, dessen Reliabilität in diesen Fällen schlecht war. Diese Daten tragen dazu bei, die Kenntnisse von Epidemiologie und Resistenzlage von Hefen für Deutschland zu präzisieren. Weitere nationale Überwachungsstudien sind unentbehrlich, um neue Tendenzen von Resistenzbildungen rasch zu entdecken, deren Mechanismen besser zu verstehen und die neuerworbenen Kenntnisse schnellstmöglich in der Klinik zu implementieren. 


\section{$6 \quad$ Zusammenfassung}

Systemische Pilzinfektionen stellen durch steigende Inzidenz und hohe Mortalität ein relevantes Problem unseres Gesundheitssystems dar. Die vorhandene Forschung zur Epidemiologie und Resistenzlage in Deutschland ist bisher wenig umfangreich und untersucht nicht das gesamte Bundesgebiet.

Diese Arbeit hat insgesamt 567 Isolate der Stammsammlung des Nationalen Referenzzentrums für systemische Mykosen in Göttingen aus dem Zeitraum Juli 2004 bis August 2005 untersucht. Die Isolate stammten aus ganz Deutschland, so dass für Deutschland repräsentative Auswertungen vorliegen.

Ziel der Arbeit war a) einen Überblick über die Epidemiologie systemischer Pilzinfektionen in Deutschland zu gewinnen; b) mittels Mikrodilutionstest als Referenzmethode die Resistenzlage gegenüber den sechs häufig eingesetzten Antimykotika Fluconazol, Itraconazol, Voriconazol, Caspofungin, Amphotericin B sowie 5-Fluorcytosin zu untersuchen sowie c) den Etest mit der Referenzmethode zu vergleichen.

Die untersuchten Pilze waren aus primär sterilen Materialien isoliert worden. $40,6 \%$ der Isolate stammten von Patienten und Patientinnen, die intensivmedizinisch behandelt wurden, weitere $16 \%$ wurden internistisch und $7,6 \%$ chirurgisch behandelt. Aus den Isolaten wurden insgesamt 21 verschiedene Spezies isoliert. Wichtigste Erreger einer systemischen Pilzinfektion sind die Spezies C. albicans $(56,8 \%)$, C. glabrata $(18,5 \%)$, C. parapsilosis $(8,1 \%)$, C. tropicalis $(7,4 \%)$ sowie C. kefyr $(1,9 \%)$ und C. krusei $(1,4 \%)$. Der Non-albicansAnteil lag bei $43,2 \%$. Mehr Isolate wurden von männlichen als von weiblichen Patienten gewonnen; das Verhältnis lag bei $1: 0,8$. Verschiedene Altersgruppen wiesen jedoch Besonderheiten in der Speziesverteilung auf. Beispielsweise war C. parapsilosis in der Altersgruppe der unter 5-Jährigen sowie in der Gruppe der 66- bis 75-Jährigen prädominant.

Die Resistenztestung aller in die Untersuchung eingeschlossenen Pilzisolate mittels Mikrodilutionstest nach CLSI gegenüber den sechs Antimykotika zeigte die folgenden Ergebnisse: Gegenüber Fluconazol waren 90,8\% der Isolate empfindlich. Bei Itraconazol lag der Anteil empfindlicher Isolate bei $63 \%$. Im 
Besonderen resistent waren die Spezies C. glabrata (Anteil empfindlicher Isolate $3,8 \%$ ) und C. krusei (Anteil empfindlicher Isolate 0\%). Für Voriconazol ergaben die Auswertungen eine Empfindlichkeit von 94,4\%. Zur Bewertung der Caspofungin-Ergebnisse wurden die kürzlich publizierten Breakpoints herangezogen. 96,8\% der Isolate waren empfindlich. Alle Isolate waren sensibel gegenüber Amphotericin B. 93,6\% der Isolate waren empfindlich gegenüber 5-Fluorcytosin.

Der Vergleich des Etests mit der Referenzmethode anhand einer ausgewählten Anzahl an Pilzisolaten zeigte die folgenden Ergebnisse: $82 \%$ der per Etest gegenüber Fluconazol getesteten Isolate wurden als empfindlich eingestuft. Übereinstimmung zur Referenzmethode in Bezug auf die Zuordnung zu den Kategorien „sensibel“, „intermediär“ oder „resistent“ (kategoriale Übereinstimmung) lag in $85,2 \%$ vor; hinsichtlich der minimalen Hemmkonzentrationen (MHK) lag eine Übereinstimmung innerhalb von zwei Konzentrationsstufen von 91,4\% vor. Die durch den Etest gegenüber Itraconazol untersuchten Isolate waren in 55,2\% empfindlich. Die Korrelation in Bezug auf die Zuordnung zu den Kategorien lag bei $63,3 \%$, die hinsichtlich der MHK bei $55,5 \%$. Mit dem Etest wurden $92,5 \%$ der Isolate gegenüber Voriconazol als empfindlich getestet. Die Übereinstimmung hinsichtlich der Kategorien lag bei 90\%, die hinsichtlich der MHK bei 86,2\%. Zur Bewertung der CaspofunginErgebnisse wurden wiederum die kürzlich publizierten Breakpoints herangezogen. Der Etest wies eine Empfindlichkeit in 98,1\% der Isolate nach. Die kategoriale Übereinstimmung lag bei $97,5 \%$, die der MHK bei $87,4 \%$. Auch im Etest waren alle Isolate sensibel gegenüber Amphotericin B. Die kategoriale Übereinstimmung lag bei 100\%. 90,8\% der durch Etest gegenüber 5-Fluorcytosin getesteten Isolate waren sensibel. Die kategoriale Korrelation lag bei $94,9 \%$, die in Bezug auf die MHK bei 93,8\%. Hervorzuheben war die hohe Resistenzrate gegenüber 5-Fluorcytosin bei der Spezies C. tropicalis mit $59,5 \%$ in beiden Testmethoden.

Diese Arbeit gibt somit einen relevanten Überblick über die Epidemiologie systemischer Pilzinfektionen in Deutschland. Außerdem wurde die Resistenzlage der isolierten Pilze bei systemischen Mykosen durch zwei Testverfahren ermittelt. 
Vor dem Hintergrund der hohen Mortalität bei diesen Infektionen ist die Fortführung von Überwachungsstudien wie dieser unerlässlich für die Anpassung von Therapieregimen an Veränderungen von Speziesvorkommen und Resistenzen. Ziel ist eine raschere, effektivere und besser verträgliche Therapie für Patientinnen und Patienten. 


\section{$7 \quad$ Anhang}

Tabelle 7-1: Speziesverteilung der Isolate in den verschiedenen Altersgruppen bei männlichen ( $\mathrm{m}$ ) und weiblichen (w) Patienten

\begin{tabular}{|c|c|c|c|c|c|c|c|c|c|c|}
\hline & & $\begin{array}{l}\text { Alle } \\
\text { rgänge }\end{array}$ & $\begin{array}{c}2000- \\
2005\end{array}$ & $\begin{array}{c}1980- \\
1999\end{array}$ & $\begin{array}{c}1970- \\
1979\end{array}$ & $\begin{array}{c}1960- \\
1969\end{array}$ & $\begin{array}{l}1950- \\
1959\end{array}$ & $\begin{array}{c}1940- \\
1949\end{array}$ & $\begin{array}{c}1930- \\
1939\end{array}$ & $\begin{array}{l}1906 \\
1929\end{array}$ \\
\hline \multirow{3}{*}{ Gesamt } & & 566 & 14 & 21 & 26 & 37 & 75 & 118 & 171 & 104 \\
\hline & $\mathrm{m}$ & 310 & 7 & 8 & 12 & 22 & 42 & 71 & 99 & 49 \\
\hline & w & 256 & 7 & 13 & 14 & 15 & 33 & 47 & 72 & 55 \\
\hline \multirow{2}{*}{ C. albicans } & $\mathrm{m}$ & 188 & 3 & 7 & 7 & 11 & 29 & 49 & 54 & 28 \\
\hline & $w$ & 137 & 1 & 2 & 10 & 11 & 19 & 34 & 31 & 29 \\
\hline \multirow{2}{*}{ C. glabrata } & $\mathrm{m}$ & 56 & 1 & - & 1 & 3 & 7 & 12 & 16 & 16 \\
\hline & w & 48 & 1 & 6 & 3 & 1 & 9 & 8 & 7 & 13 \\
\hline \multirow[t]{2}{*}{ C. parapsilosis } & $\mathrm{m}$ & 15 & 2 & 1 & 1 & 2 & 3 & 1 & 5 & - \\
\hline & w & 30 & 3 & 1 & - & 1 & 2 & 1 & 17 & 5 \\
\hline \multirow[t]{2}{*}{ C. tropicalis } & $\mathrm{m}$ & 25 & - & - & 1 & 1 & 1 & 4 & 16 & 2 \\
\hline & $w$ & 15 & 1 & 1 & - & 1 & 2 & 3 & 3 & 4 \\
\hline \multirow{2}{*}{ C. kefyr } & $\mathrm{m}$ & - & & & & & & - & - & - \\
\hline & $w$ & 11 & & & & & & 1 & 8 & 2 \\
\hline \multirow[t]{2}{*}{ C. krusei } & $\mathrm{m}$ & 4 & - & - & & & 1 & 1 & 2 & \\
\hline & $w$ & 4 & 1 & 1 & & & - & - & 2 & \\
\hline \multirow[t]{2}{*}{ C. guilliermondii } & $\mathrm{m}$ & 4 & & & & 1 & & 2 & & 1 \\
\hline & w & - & & & & - & & - & & - \\
\hline \multirow[t]{2}{*}{ C. dubliniensis } & $\mathrm{m}$ & 2 & & & 1 & - & & 1 & & \\
\hline & w & 1 & & & - & 1 & & - & & \\
\hline \multirow[t]{2}{*}{ C. inconspicua } & $\mathrm{m}$ & 2 & & & & & & & 2 & \\
\hline & w & 1 & & & & & & & 1 & \\
\hline \multirow[t]{2}{*}{ C. Iusitaniae } & $\mathrm{m}$ & 1 & & & & & 1 & & & \\
\hline & w & - & & & & & - & & & \\
\hline \multirow[t]{2}{*}{ C. norvegensis } & $\mathrm{m}$ & 1 & & & & & - & & 1 & \\
\hline & w & 1 & & & & & 1 & & - & \\
\hline \multirow[t]{2}{*}{ C. catenulata } & $\mathrm{m}$ & 1 & & & & & & & 1 & \\
\hline & w & - & & & & & & & - & \\
\hline \multirow[t]{2}{*}{ C. colliculosa } & $\mathrm{m}$ & - & & & & & & & - & \\
\hline & w & 1 & & & & & & & 1 & \\
\hline \multirow[t]{2}{*}{ C. famata } & $\mathrm{m}$ & 2 & 1 & & & & & 1 & & \\
\hline & w & - & - & & & & & - & & \\
\hline \multirow[t]{2}{*}{ C. intermedia } & $\mathrm{m}$ & - & & - & & & & & & \\
\hline & w & 1 & & 1 & & & & & & \\
\hline \multirow[t]{2}{*}{ C. rugosa } & $\mathrm{m}$ & - & & & - & & & & & \\
\hline & w & 1 & & & 1 & & & & & \\
\hline \multirow[t]{2}{*}{ C. utilis } & $\mathrm{m}$ & - & & & & & & & & - \\
\hline & w & 1 & & & & & & & & 1 \\
\hline \multirow[t]{2}{*}{ Cr. neoformans } & $\mathrm{m}$ & 5 & & & 1 & 3 & & & 1 & \\
\hline & w & - & & & - & - & & & - & \\
\hline \multirow[t]{2}{*}{ G. capitatum } & $\mathrm{m}$ & - & & - & & & & & & \\
\hline & w & 1 & & 1 & & & & & & \\
\hline \multirow[t]{2}{*}{ S. cerevisiae } & $\mathrm{m}$ & 2 & & & & 1 & & & - & 1 \\
\hline & w & 3 & & & & - & & & 2 & 1 \\
\hline Trichosporon & $\mathrm{m}$ & 2 & & & & & & & 1 & 1 \\
\hline spp. & w & - & & & & & & & - & - \\
\hline
\end{tabular}


Tabelle 7-2 bis 7-7: Darstellung der erhaltenen Mikrodilutions- (M) und Etest- (E) Ergebnisse in jeder MHK-Stufe (in $\mu \mathrm{g} / \mathrm{ml}$ ) für die sechs Antimykotika als Anzahl der Isolate mit Angabe der Bewertungskategorien sensibel, dosisabhängig sensibel (dsensibel) und resistent. Für Caspofungin sind die intermediären Konzentrationsbereiche speziesabhängig grau hinterlegt (soweit vorhanden) bzw. sind die Bereiche sensibel und resistent mit schwarzem Balken voneinander getrennt. 
Tabelle 7-2: Fluconazol

\begin{tabular}{|c|c|c|c|c|c|c|c|c|c|c|c|c|}
\hline \multirow[b]{3}{*}{ Fluconazol } & \multirow{3}{*}{$\stackrel{\oplus}{\mathscr{g}}$} & \multicolumn{10}{|c|}{ Anzahl der Isolate mit der jeweiligen MHK } & \multirow[b]{3}{*}{$\mathbf{N}$} \\
\hline & & \multicolumn{7}{|c|}{ sensibel } & \multicolumn{2}{|c|}{ d-sensibel } & \multirow{2}{*}{$\begin{array}{c}\text { resistent } \\
>32\end{array}$} & \\
\hline & & $<0,25$ & 0,25 & 0,5 & 1 & 2 & 4 & 8 & 16 & 32 & & \\
\hline \multirow[b]{2}{*}{ Gesamt } & $\mathbf{M}$ & 71 & 152 & 113 & 55 & 34 & 62 & 28 & 23 & 9 & 20 & 567 \\
\hline & $E$ & 32 & 117 & 150 & 106 & 16 & 14 & 31 & 52 & 17 & 32 & 567 \\
\hline \multirow[b]{2}{*}{ C. albicans } & $\mathbf{M}$ & 62 & 130 & 65 & 36 & 9 & 4 & 5 & 1 & 1 & 9 & 322 \\
\hline & $\mathbf{E}$ & 26 & 98 & 114 & 65 & 7 & 3 & 2 & 2 & & 5 & 322 \\
\hline \multirow[b]{2}{*}{ C. glabrata } & $\mathbf{M}$ & 3 & 2 & 1 & & 10 & 49 & 19 & 12 & 3 & 6 & 105 \\
\hline & $E$ & & 3 & 2 & & 1 & 6 & 24 & 42 & 12 & 15 & 105 \\
\hline \multirow[b]{2}{*}{ C. parapsilosis } & $\mathbf{M}$ & 2 & 2 & 24 & 8 & 6 & 1 & 1 & 1 & 1 & & 46 \\
\hline & $\mathbf{E}$ & 1 & 6 & 17 & 18 & 3 & & & 1 & 1 & & 46 \\
\hline \multirow[b]{2}{*}{ C. tropicalis } & $M$ & & 12 & 12 & 8 & 6 & 1 & & & 1 & 2 & 42 \\
\hline & $E$ & & 3 & 17 & 16 & 3 & 2 & & & & 1 & 42 \\
\hline \multirow[b]{2}{*}{ C. kefyr } & $\mathbf{M}$ & & 3 & 8 & & & & & & & & 11 \\
\hline & $E$ & 3 & 6 & & 2 & & & & & & & 11 \\
\hline \multirow[b]{2}{*}{ C. krusei } & $M$ & & & & & & & 1 & 4 & 1 & 2 & 8 \\
\hline & $E$ & & & & & & & & 3 & 1 & 4 & 8 \\
\hline \multirow[b]{2}{*}{ C. guillierm. } & $\mathbf{M}$ & & & & & 1 & 2 & & 1 & & & 4 \\
\hline & $\mathbf{E}$ & & & & 1 & 1 & 1 & 1 & & & & 4 \\
\hline \multirow[b]{2}{*}{ C. dubliniensis } & $M$ & 2 & 1 & & & & & & & & & 3 \\
\hline & $\mathbf{E}$ & 1 & 1 & & 1 & & & & & & & 3 \\
\hline \multirow[b]{2}{*}{ C. inconspicua } & $\mathbf{M}$ & & & & & & & & 3 & & & 3 \\
\hline & $E$ & & & & & & & & 1 & 1 & 1 & 3 \\
\hline \multirow[b]{2}{*}{ C. Iusitaniae } & $\mathbf{M}$ & & 1 & & 1 & & & & & & & 2 \\
\hline & $\mathbf{E}$ & & & & 2 & & & & & & & 2 \\
\hline \multirow[b]{2}{*}{ C. norvegensis } & $\mathbf{M}$ & & & & 1 & & & & & & 1 & 2 \\
\hline & $E$ & & & & & & & & 2 & & & 2 \\
\hline & $M$ & 1 & & & & & & & & & & 1 \\
\hline C. catenulata & $E$ & 1 & & & & & & & & & & 1 \\
\hline & $M$ & 1 & & & & & & & & & & 1 \\
\hline C. colliculosa & $E$ & & 1 & & & & & & & & & 1 \\
\hline & $\mathbf{M}$ & & & & 1 & & & & & & & 1 \\
\hline C. famata & $\mathbf{E}$ & & & & & & 1 & & & & & 1 \\
\hline & $M$ & & 1 & & & & & & & & & 1 \\
\hline C. intermedia & $E$ & & & 1 & & & & & & & & 1 \\
\hline & $\mathbf{M}$ & & & 1 & & & & & & & & 1 \\
\hline C. rugosa & $E$ & & & & & & & 1 & & & & 1 \\
\hline & $M$ & & & & & & 1 & & & & & 1 \\
\hline C. utilis & $E$ & & & & & & & 1 & & & & 1 \\
\hline & $\mathbf{M}$ & & & & & 2 & 2 & 1 & & & & 5 \\
\hline Cr. neoformans & $E$ & & & & & & 1 & 1 & & 2 & 1 & 5 \\
\hline & $M$ & & & & & & & & & 1 & & 1 \\
\hline G. capitatum & E & & & & & & & & 1 & & & 1 \\
\hline & $M$ & & & & & & 2 & 1 & 1 & 1 & & 5 \\
\hline S. cerevisiae & $E$ & & & & & & & 1 & & & 4 & 5 \\
\hline & $\mathbf{M}$ & & & 1 & & 1 & & & & & & 2 \\
\hline Trichosp. spp. & $E$ & & & & 1 & 1 & & & & & & 2 \\
\hline
\end{tabular}


Tabelle 7-3: Itraconazol

\begin{tabular}{|c|c|c|c|c|c|c|c|c|c|c|c|c|c|c|}
\hline \multirow[b]{3}{*}{ Itraconazol } & \multirow{3}{*}{ 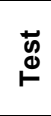 } & \multicolumn{12}{|c|}{ Anzahl der Isolate mit der jeweiligen MHK } & \multirow[b]{3}{*}{$\mathbf{N}$} \\
\hline & & \multicolumn{4}{|c|}{ sensibel } & \multicolumn{2}{|c|}{ d-sensibel } & \multicolumn{6}{|c|}{ resistent } & \\
\hline & & $<0,0313$ & 0,0313 & 0,0625 & 0,125 & 0,25 & 0,5 & 1 & 2 & 4 & 8 & 16 & $>16$ & \\
\hline \multirow[b]{2}{*}{ Gesamt } & M & 1 & 48 & 143 & 165 & 58 & 51 & 63 & 5 & 4 & & 29 & & 567 \\
\hline & $\mathbf{E}$ & 38 & 70 & 40 & 28 & 11 & 14 & 13 & 7 & 16 & 19 & 10 & 53 & 319 \\
\hline \multirow[b]{2}{*}{ C. albicans } & $\mathbf{M}$ & & 45 & 127 & 111 & 15 & 4 & 1 & & 2 & & 17 & & 322 \\
\hline & $\mathrm{E}$ & 25 & 45 & 15 & 6 & 1 & 1 & & & 1 & 2 & & 2 & 97 \\
\hline \multirow[b]{2}{*}{ C. glabrata } & $M$ & 1 & 1 & 2 & & 7 & 32 & 49 & 4 & 1 & & 8 & & 105 \\
\hline & $E$ & 1 & 2 & 1 & & & 2 & 5 & 7 & 14 & 16 & 10 & 46 & 104 \\
\hline \multirow[b]{2}{*}{ C. parapsilosis } & $M$ & & & 5 & 29 & 9 & 2 & 1 & & & & & & 46 \\
\hline & $E$ & 6 & 8 & 12 & 16 & 2 & 1 & 1 & & & & & & 46 \\
\hline \multirow[b]{2}{*}{ C. tropicalis } & $M$ & & 1 & 5 & 15 & 13 & 5 & & & 1 & & 2 & & 42 \\
\hline & $E$ & 5 & 12 & 6 & 4 & 5 & 4 & 5 & & & & & 1 & 42 \\
\hline \multirow[b]{2}{*}{ C. kefyr } & $M$ & & & 1 & 2 & 8 & & & & & & & & 11 \\
\hline & $E$ & 1 & 2 & 4 & & & & & & & & & & 7 \\
\hline \multirow[b]{2}{*}{ C. krusei } & $\mathbf{M}$ & & & & & 1 & 2 & 5 & & & & & & 8 \\
\hline & $E$ & & & & 2 & & 3 & 1 & & & 1 & & & 7 \\
\hline \multirow[b]{2}{*}{ C. guillierm. } & $M$ & & & & & 2 & 1 & & 1 & & & & & 4 \\
\hline & $E$ & & & & & & & & & & & & & - \\
\hline \multirow[b]{2}{*}{ C. dubliniensis } & $M$ & & & 3 & & & & & & & & & & 3 \\
\hline & $E$ & & & & & & & & & & & & & - \\
\hline \multirow[b]{2}{*}{ C. inconspicua } & $\mathbf{M}$ & & & & & 1 & 1 & 1 & & & & & & 3 \\
\hline & $E$ & & & & & 1 & 1 & 1 & & & & & & 3 \\
\hline \multirow[b]{2}{*}{ C. Iusitaniae } & $M$ & & & & 1 & & 1 & & & & & & & 2 \\
\hline & $E$ & & & & & & & & & & & & & - \\
\hline & $M$ & & & & & 1 & 1 & & & & & & & 2 \\
\hline C. norvegensis & $E$ & & & & & 1 & 1 & & & & & & & 2 \\
\hline & $M$ & & & & 1 & & & & & & & & & 1 \\
\hline C. catenulata & $E$ & & & & & & & & & & & & & - \\
\hline & $M$ & & & & 1 & & & & & & & & & 1 \\
\hline C. colliculosa & $E$ & & & & & & & & & & & & & - \\
\hline & $\mathbf{M}$ & & & & & 1 & & & & & & & & 1 \\
\hline C. famata & $E$ & & & & & & & & & & & & & - \\
\hline & $\mathbf{M}$ & & & & 1 & & & & & & & & & 1 \\
\hline C. intermedia & $E$ & & & & & & & & & & & & & - \\
\hline & $\mathbf{M}$ & & & & & & & & & & & 1 & & 1 \\
\hline C. rugosa & $E$ & & & & & & & & & & & & & - \\
\hline & $\mathbf{M}$ & & & & & & & 1 & & & & & & 1 \\
\hline C. utilis & $E$ & & & & & & & & & 1 & & & & 1 \\
\hline & $\mathbf{M}$ & & & & 2 & 2 & 1 & & & & & & & 5 \\
\hline Cr. neoformans & $E$ & & 1 & 1 & 1 & 1 & & & & & & & & 4 \\
\hline & M & & & & & & & 1 & & & & & & 1 \\
\hline G. capitatum & $E$ & & & 1 & & & & & & & & & & 1 \\
\hline & $M$ & & & & & & 1 & 3 & & & & 1 & & 5 \\
\hline S. cerevisiae & $E$ & & & & & & & & & & & & 4 & 4 \\
\hline & $M$ & & 1 & & 1 & & & & & & & & & 2 \\
\hline Trichosp. spp. & $E$ & & & & & & 1 & & & & & & & 1 \\
\hline
\end{tabular}


Tabelle 7-4: Voriconazol

\begin{tabular}{|c|c|c|c|c|c|c|c|c|c|c|c|c|c|c|}
\hline \multirow[b]{3}{*}{ Voriconazol } & \multirow{3}{*}{$\underset{\mathscr{d}}{\stackrel{4}{㇒}}$} & \multicolumn{12}{|c|}{ Anzahl der Isolate mit der jeweiligen MHK } & \multirow[b]{3}{*}{$\mathbf{N}$} \\
\hline & & \multicolumn{6}{|c|}{ sensibel } & \multicolumn{2}{|c|}{ d-sensibel } & \multicolumn{4}{|c|}{ resistent } & \\
\hline & & $<0,0313$ & 0,0313 & 0,0625 & 0,125 & 0,25 & 0,5 & 1 & 2 & 4 & 8 & 16 & $>16$ & \\
\hline \multirow[b]{2}{*}{ Gesamt } & $\mathbf{M}$ & 247 & 115 & 74 & 62 & 23 & 14 & 10 & 3 & 5 & 1 & 13 & & 567 \\
\hline & $\mathbf{E}$ & 138 & 36 & 20 & 52 & 29 & 20 & 8 & 5 & 4 & 4 & & 3 & 319 \\
\hline \multirow[b]{2}{*}{ C. albicans } & $\mathbf{M}$ & 215 & 70 & 14 & 7 & 3 & 3 & 1 & & 1 & & 8 & & 322 \\
\hline & $\mathbf{E}$ & 83 & 10 & 1 & 1 & 1 & & 1 & & 1 & & & & 98 \\
\hline \multirow[b]{2}{*}{ C. glabrata } & $\mathbf{M}$ & 3 & 2 & 18 & 48 & 15 & 6 & 7 & 2 & 4 & & & & 105 \\
\hline & $\mathbf{E}$ & 3 & 2 & 3 & 36 & 21 & 17 & 7 & 5 & 3 & 4 & & 2 & 103 \\
\hline \multirow[b]{2}{*}{ C. parapsilosis } & $\mathbf{M}$ & 11 & 9 & 21 & 2 & 2 & & & & & & 1 & & 46 \\
\hline & $\mathbf{E}$ & 36 & 6 & 2 & 1 & & & & & & & & & 45 \\
\hline \multirow[b]{2}{*}{ C. tropicalis } & M & 5 & 19 & 8 & 1 & 1 & 2 & & & & 1 & 5 & & 42 \\
\hline & $E$ & 9 & 16 & 10 & 5 & 1 & & & & & & & 1 & 42 \\
\hline \multirow[b]{2}{*}{ C. kefyr } & $\mathbf{M}$ & 5 & 1 & 5 & & & & & & & & & & 11 \\
\hline & $\mathbf{E}$ & 6 & 1 & & & & & & & & & & & 7 \\
\hline \multirow[b]{2}{*}{ C. krusei } & $\mathbf{M}$ & & & 1 & 2 & 2 & 1 & 2 & & & & & & 8 \\
\hline & $E$ & & & & 3 & 4 & 1 & & & & & & & 8 \\
\hline \multirow[b]{2}{*}{ C. guillierm. } & $\mathbf{M}$ & & 1 & 2 & & & & & 1 & & & & & 4 \\
\hline & $E$ & & & & & & & & & & & & & - \\
\hline \multirow[b]{2}{*}{ C. dubliniensis } & $\mathbf{M}$ & 3 & & & & & & & & & & & & 3 \\
\hline & $\mathbf{E}$ & & & & & & & & & & & & & - \\
\hline \multirow[b]{2}{*}{ C. inconspicua } & $\mathbf{M}$ & 1 & & 1 & 1 & & & & & & & & & 3 \\
\hline & $\mathbf{E}$ & & & & 2 & 1 & & & & & & & & 3 \\
\hline \multirow[b]{2}{*}{ C. Iusitaniae } & M & 1 & & 1 & & & & & & & & & & 2 \\
\hline & $\mathbf{E}$ & & & & & & & & & & & & & - \\
\hline & $\mathbf{M}$ & & & & & & 1 & 1 & & & & & & 2 \\
\hline C. norvegensis & E & & & & 1 & 1 & & & & & & & & 2 \\
\hline & $\mathbf{M}$ & & 1 & & & & & & & & & & & 1 \\
\hline C. catenulata & E & & & & & & & & & & & & & - \\
\hline & $\mathbf{M}$ & & 1 & & & & & & & & & & & 1 \\
\hline C. colliculosa & $\mathbf{E}$ & & & & & & & & & & & & & - \\
\hline & $M$ & & & 1 & & & & & & & & & & 1 \\
\hline C. famata & $\mathbf{E}$ & & & & & & & & & & & & & - \\
\hline & $\mathbf{M}$ & 1 & & & & & & & & & & & & 1 \\
\hline C. intermedia & $\mathbf{E}$ & & & & & & & & & & & & & - \\
\hline & $\mathbf{M}$ & & & & & & & & & & & 1 & & 1 \\
\hline C. rugosa & $\mathbf{E}$ & & & & & & & & & & & & & - \\
\hline & $\mathbf{M}$ & & & & & & 1 & & & & & & & 1 \\
\hline C. utilis & $E$ & & & & & & 1 & & & & & & & 1 \\
\hline & $\mathbf{M}$ & & 4 & 1 & & & & & & & & & & 5 \\
\hline Cr. neoformans & $\mathbf{E}$ & 1 & & 1 & 1 & & 1 & & & & & & & 4 \\
\hline & M & & & & & & 1 & & & & & & & 1 \\
\hline G. capitatum & $\mathbf{E}$ & & 1 & & & & & & & & & & & 1 \\
\hline & M & & 5 & & & & & & & & & & & 5 \\
\hline S. cerevisiae & $\mathbf{E}$ & & & 2 & 2 & & & & & & & & & 4 \\
\hline & M & & 2 & & & & & & & & & & & 2 \\
\hline Trichosp. spp. & $\mathbf{E}$ & & & 1 & & & & & & & & & & 1 \\
\hline
\end{tabular}


Tabelle 7-5: Caspofungin

\begin{tabular}{|c|c|c|c|c|c|c|c|c|c|c|c|c|c|c|}
\hline \multirow[b]{2}{*}{ Caspofungin } & \multirow{2}{*}{$\begin{array}{l}\mathbf{w} \\
\stackrel{0}{\circ} \\
\end{array}$} & \multicolumn{12}{|c|}{ Anzahl der Isolate mit der jeweiligen MHK } & \multirow[b]{2}{*}{$\mathbf{N}$} \\
\hline & & $<0,0313$ & 0,0313 & 0,0625 & 0,125 & 0,25 & 0,5 & 1 & 2 & 4 & 8 & 16 & $>16$ & \\
\hline \multirow[b]{2}{*}{ Gesamt } & M & 137 & 238 & 96 & 28 & 36 & 17 & 3 & & & 6 & 6 & & 567 \\
\hline & $\mathbf{E}$ & 60 & 28 & 34 & 127 & 31 & 26 & 5 & 1 & & & & 6 & 318 \\
\hline \multirow[b]{2}{*}{ C. albicans } & $\mathbf{M}$ & 120 & 143 & 50 & 7 & 2 & & & & & & & & 322 \\
\hline & $\mathbf{E}$ & 11 & 5 & 19 & 55 & 9 & 1 & & & & & & & 100 \\
\hline \multirow[b]{2}{*}{ C. glabrata } & M & 3 & 66 & 28 & 2 & 5 & 1 & & & & & & & 105 \\
\hline & $E$ & 27 & 16 & 8 & 49 & 1 & & & & & & & & 101 \\
\hline \multirow[b]{2}{*}{ C. parapsilosis } & $\mathbf{M}$ & & 1 & 1 & 6 & 25 & 13 & & & & & & & 46 \\
\hline & $E$ & & & & 1 & 15 & 24 & 5 & 1 & & & & & 46 \\
\hline \multirow[b]{2}{*}{ C. tropicalis } & $\mathbf{M}$ & 9 & 22 & 6 & 4 & 1 & & & & & & & & 42 \\
\hline & $E$ & 9 & 7 & 6 & 14 & 3 & 1 & & & & & & & 40 \\
\hline \multirow[b]{2}{*}{ C. kefyr } & M & 4 & 1 & 6 & & & & & & & & & & 11 \\
\hline & $\mathbf{E}$ & 7 & & & & & & & & & & & & 7 \\
\hline \multirow[b]{2}{*}{ C. krusei } & $M$ & & & 2 & 5 & 1 & & & & & & & & 8 \\
\hline & $E$ & 1 & & & 5 & 2 & & & & & & & & 8 \\
\hline \multirow[b]{2}{*}{ C. guillierm. } & $\mathbf{M}$ & & & & & & & & & & & 4 & & 4 \\
\hline & $E$ & & & & & & & & & & & & & - \\
\hline \multirow[b]{2}{*}{ C. dubliniensis } & $M$ & 1 & 1 & & 1 & & & & & & & & & 3 \\
\hline & $E$ & & & & & & & & & & & & & - \\
\hline \multirow[b]{2}{*}{ C. inconspicua } & $\mathbf{M}$ & & & 1 & & & 2 & & & & & & & 3 \\
\hline & $E$ & 2 & & & 1 & & & & & & & & & 3 \\
\hline \multirow[b]{2}{*}{ C. lusitaniae } & $\mathbf{M}$ & & & & 1 & 1 & & & & & & & & 2 \\
\hline & $E$ & & & & & & & & & & & & & - \\
\hline \multirow[b]{2}{*}{ C. norvegensis } & $M$ & & & 1 & 1 & & & & & & & & & 2 \\
\hline & $E$ & 2 & & & & & & & & & & & & 2 \\
\hline \multirow[b]{2}{*}{ C. catenulata } & $M$ & & & & & & & 1 & & & & & & 1 \\
\hline & $E$ & & & & & & & & & & & & & - \\
\hline \multirow[b]{2}{*}{ C. colliculosa } & $\mathbf{M}$ & & & & & 1 & & & & & & & & 1 \\
\hline & $E$ & & & & & & & & & & & & & - \\
\hline \multirow[b]{2}{*}{ C. famata } & $M$ & & & & & & & 1 & & & 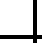 & & & 1 \\
\hline & $\mathbf{E}$ & & & & & & & & & & & & & - \\
\hline & $M$ & & & & & & 1 & & & & & & & 1 \\
\hline C. intermedia & $E$ & & & & & & & & & & & & & - \\
\hline & $\mathbf{M}$ & & & & & & & 1 & & & & & & 1 \\
\hline C. rugosa & $E$ & & & & & & & & & & & & & - \\
\hline & $\mathbf{M}$ & & & & 1 & & & & & & 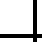 & & & 1 \\
\hline C. utilis & $E$ & 1 & & & & & & & & & & & & 1 \\
\hline & $M$ & & & & & & & & & & 4 & 1 & & 5 \\
\hline Cr. neoformans & $E$ & & & & & & & & & & & & 4 & 4 \\
\hline & $M$ & & & & & & & & & & 1 & & & 1 \\
\hline G. capitatum & $E$ & & & & & & & & & & & & 1 & 1 \\
\hline & $\mathbf{M}$ & & 4 & 1 & & & & & & & & & & 5 \\
\hline S. cerevisiae & $E$ & & & 1 & 2 & 1 & & & & & & & & 4 \\
\hline & $M$ & & & & & & & & & & 1 & 1 & & 2 \\
\hline Trichosp. spp. & $E$ & & & & & & & & & & & & 1 & 1 \\
\hline
\end{tabular}


Tabelle 7-6: Amphotericin B

\begin{tabular}{|c|c|c|c|c|c|c|c|c|c|c|c|c|c|}
\hline \multirow[b]{3}{*}{ Amphotericin B } & \multirow{3}{*}{$\stackrel{\ddot{g}}{\stackrel{H}{r}}$} & \multicolumn{11}{|c|}{ Anzahl der Isolate mit der jeweiligen MHK } & \multirow[b]{3}{*}{$\mathbf{N}$} \\
\hline & & \multicolumn{7}{|c|}{ sensibel } & \multicolumn{4}{|c|}{ resistent } & \\
\hline & & $<0,0313$ & 0,0313 & 0,0625 & 0,13 & 0,25 & 0,5 & 1 & 2 & 4 & 8 & 16 & \\
\hline \multirow[b]{2}{*}{ Gesamt } & $\mathbf{M}$ & & & 3 & 6 & 32 & 292 & 234 & & & & & 567 \\
\hline & $\mathbf{E}$ & 120 & 79 & 57 & 45 & 13 & 1 & 3 & & & & & 318 \\
\hline \multirow[b]{2}{*}{ C. albicans } & $M$ & & & 1 & 5 & 18 & 224 & 74 & & & & & 322 \\
\hline & $E$ & 46 & 23 & 16 & 8 & 2 & & & & & & & 95 \\
\hline \multirow[b]{2}{*}{ C. glabrata } & $\mathbf{M}$ & & & & & 2 & 25 & 78 & & & & & 105 \\
\hline & $\mathbf{E}$ & 27 & 29 & 21 & 19 & 5 & & 3 & & & & & 104 \\
\hline \multirow[b]{2}{*}{ C. parapsilosis } & $M$ & & & & & 1 & 18 & 27 & & & & & 46 \\
\hline & $\mathbf{E}$ & 14 & 10 & 13 & 9 & & & & & & & & 46 \\
\hline \multirow[b]{2}{*}{ C. tropicalis } & $\mathbf{M}$ & & & & & 4 & 11 & 27 & & & & & 42 \\
\hline & $\mathbf{E}$ & 21 & 13 & 3 & 4 & & 1 & & & & & & 42 \\
\hline \multirow[b]{2}{*}{ C. kefyr } & $M$ & & & & & & 1 & 10 & & & & & 11 \\
\hline & $\mathbf{E}$ & & & & 3 & 4 & & & & & & & 7 \\
\hline \multirow[b]{2}{*}{ C. krusei } & $M$ & & & & & & 3 & 5 & & & & & 8 \\
\hline & $E$ & 2 & & 3 & 1 & 2 & & & & & & & 8 \\
\hline \multirow[b]{2}{*}{ C. guillierm. } & $M$ & & & & & & 2 & 2 & & & & & 4 \\
\hline & $\mathbf{E}$ & & & & & & & & & & & & - \\
\hline \multirow[b]{2}{*}{ C. dubliniensis } & $M$ & & & & & 3 & & & & & & & 3 \\
\hline & $\mathbf{E}$ & & & & & & & & & & & & - \\
\hline \multirow[b]{2}{*}{ C. inconspicua } & $\mathbf{M}$ & & & 1 & & & 2 & & & & & & 3 \\
\hline & $\mathbf{E}$ & 2 & & & 1 & & & & & & & & 3 \\
\hline \multirow[b]{2}{*}{ C. Iusitaniae } & $M$ & & & & & & 1 & 1 & & & & & 2 \\
\hline & $\mathbf{E}$ & & & & & & & & & & & & - \\
\hline \multirow[b]{2}{*}{ C. norvegensis } & $\mathbf{M}$ & & & & & 1 & 1 & & & & & & 2 \\
\hline & $\mathbf{E}$ & & 2 & & & & & & & & & & 2 \\
\hline \multirow[b]{2}{*}{ C. catenulata } & $M$ & & & & & & & 1 & & & & & 1 \\
\hline & $E$ & & & & & & & & & & & & - \\
\hline & $\mathbf{M}$ & & & & & 1 & & & & & & & 1 \\
\hline C. colliculosa & $\mathbf{E}$ & & & & & & & & & & & & - \\
\hline & $M$ & & & & & & & 1 & & & & & 1 \\
\hline C. famata & $\mathbf{E}$ & & & & & & & & & & & & - \\
\hline & M & & & & & & 1 & & & & & & 1 \\
\hline C. intermedia & $\mathbf{E}$ & & & & & & & & & & & & - \\
\hline & $\mathbf{M}$ & & & & & & & 1 & & & & & 1 \\
\hline C. rugosa & $\mathbf{E}$ & & & & & & & & & & & & - \\
\hline & $M$ & & & & 1 & & & & & & & & 1 \\
\hline C. utilis & $\mathbf{E}$ & 1 & & & & & & & & & & & 1 \\
\hline & $\mathbf{M}$ & & & & & & & 5 & & & & & 5 \\
\hline Cr. neoformans & $\mathbf{E}$ & 2 & 2 & & & & & & & & & & 4 \\
\hline & $\mathbf{M}$ & & & & & & & 1 & & & & & 1 \\
\hline G. capitatum & $\mathbf{E}$ & 1 & & & & & & & & & & & 1 \\
\hline & $\mathbf{M}$ & & & 1 & & 2 & 2 & & & & & & 5 \\
\hline S. cerevisiae & $E$ & 4 & & & & & & & & & & & 4 \\
\hline & $\mathbf{M}$ & & & & & & 1 & 1 & & & & & 2 \\
\hline Trichosp. spp. & $\mathbf{E}$ & & & 1 & & & & & & & & & 1 \\
\hline
\end{tabular}


Tabelle 7-7: 5-Fluorcytosin

\begin{tabular}{|c|c|c|c|c|c|c|c|c|c|c|c|c|}
\hline \multirow[b]{3}{*}{ 5-Fluorcytosin } & \multirow{3}{*}{$\stackrel{\ddot{g}}{\mathscr{E}}$} & \multicolumn{10}{|c|}{ Anzahl der Isolate mit der jeweiligen MHK } & \multirow[b]{3}{*}{$\mathbf{N}$} \\
\hline & & \multicolumn{7}{|c|}{ sensibel } & \multicolumn{2}{|c|}{ intermediär } & \multirow{2}{*}{$\begin{array}{c}\text { resistent } \\
>16\end{array}$} & \\
\hline & & $<0,125$ & 0,125 & 0,25 & 0,5 & 1 & 2 & 4 & 8 & 16 & & \\
\hline \multirow[b]{2}{*}{ Gesamt } & $\mathbf{M}$ & 407 & 63 & 24 & 13 & 6 & 8 & 10 & 7 & 3 & 26 & 567 \\
\hline & $\mathbf{E}$ & 391 & 74 & 16 & 19 & 9 & 4 & 2 & 3 & 3 & 46 & 567 \\
\hline \multirow[b]{2}{*}{ C. albicans } & $M$ & 258 & 37 & 15 & 11 & & & 1 & & & & 322 \\
\hline & $E$ & 240 & 56 & 8 & 11 & 2 & 3 & & & & 2 & 322 \\
\hline \multirow[b]{2}{*}{ C. glabrata } & $\mathbf{M}$ & 97 & 5 & 2 & & & 1 & & & & & 105 \\
\hline & E & 90 & 6 & 3 & 4 & & & 1 & 1 & & & 105 \\
\hline \multirow[b]{2}{*}{ C. parapsilosis } & $M$ & 23 & 16 & 4 & & 2 & 1 & & & & & 46 \\
\hline & $E$ & 33 & 6 & 3 & 2 & 1 & & & 1 & & & 46 \\
\hline \multirow[b]{2}{*}{ C. tropicalis } & $M$ & 14 & 2 & & & & & & & 1 & 25 & 42 \\
\hline & $E$ & 13 & & 1 & 1 & 2 & & & & & 25 & 42 \\
\hline \multirow[b]{2}{*}{ C. kefyr } & $M$ & 1 & & & & & 4 & 6 & & & & 11 \\
\hline & $\mathbf{E}$ & 1 & 1 & & 1 & 1 & & & & 3 & 4 & 11 \\
\hline \multirow[b]{2}{*}{ C. krusei } & $\mathbf{M}$ & & & & & & & 1 & 5 & 2 & & 8 \\
\hline & $E$ & & 1 & & & & & & & & 7 & 8 \\
\hline \multirow[b]{2}{*}{ C. guillierm. } & $M$ & 4 & & & & & & & & & & 4 \\
\hline & $E$ & 4 & & & & & & & & & & 4 \\
\hline \multirow[b]{2}{*}{ C. dubliniensis } & $\mathbf{M}$ & 3 & & & & & & & & & & 3 \\
\hline & $E$ & 3 & & & & & & & & & & 3 \\
\hline \multirow[b]{2}{*}{ C. inconspicua } & $M$ & & & & & 2 & & & 1 & & & 3 \\
\hline & $\mathbf{E}$ & & & & & 1 & & & 1 & & 1 & 3 \\
\hline \multirow[b]{2}{*}{ C. Iusitaniae } & $M$ & 1 & & 1 & & & & & & & & 2 \\
\hline & $E$ & 2 & & & & & & & & & & 2 \\
\hline \multirow[b]{2}{*}{ C. norvegensis } & $\mathbf{M}$ & & & & 1 & & & 1 & & & & 2 \\
\hline & $E$ & & & & & & 1 & & & & 1 & 2 \\
\hline & $M$ & & & 1 & & & & & & & & 1 \\
\hline C. catenulata & $E$ & & & 1 & & & & & & & & 1 \\
\hline & $\mathbf{M}$ & & 1 & & & & & & & & & 1 \\
\hline C. colliculosa & $E$ & & 1 & & & & & & & & & 1 \\
\hline & $M$ & 1 & & & & & & & & & & 1 \\
\hline C. famata & $E$ & 1 & & & & & & & & & & 1 \\
\hline & $\mathbf{M}$ & 1 & & & & & & & & & & 1 \\
\hline C. intermedia & $\mathbf{E}$ & & & & & 1 & & & & & & 1 \\
\hline & $\mathbf{M}$ & & 1 & & & & & & & & & 1 \\
\hline C. rugosa & $\mathbf{E}$ & & 1 & & & & & & & & & 1 \\
\hline & $M$ & & & & & & & & 1 & & & 1 \\
\hline C. utilis & $E$ & & & & & & & & & & 1 & 1 \\
\hline & $\mathbf{M}$ & & & & 1 & 2 & 2 & & & & & 5 \\
\hline Cr. neoformans & $E$ & & & & & 1 & & & & & 4 & 5 \\
\hline & $\mathbf{M}$ & 1 & & & & & & & & & & 1 \\
\hline G. capitatum & $E$ & 1 & & & & & & & & & & 1 \\
\hline & $\mathbf{M}$ & 3 & 1 & 1 & & & & & & & & 5 \\
\hline S. cerevisiae & $E$ & 3 & 2 & & & & & & & & & 5 \\
\hline & $M$ & & & & & & & 1 & & & 1 & 2 \\
\hline Trichosp. spp. & $E$ & & & & & & & 1 & & & 1 & 2 \\
\hline
\end{tabular}


Tabelle 7-8: Tabellarische Darstellung aller Einsender mit Adressen

\begin{tabular}{|c|c|}
\hline $\begin{array}{l}\text { Institut für Mikrobiologie und Hygiene } \\
\text { Universitätsmedizin Charité } \\
\text { Charitéplatz } 1 \\
10117 \text { Berlin }\end{array}$ & $\begin{array}{l}\text { Institut für Medizinische Mikrobiologie } \\
\text { Universitätsklinikum Carl Gustav Carus an } \\
\text { der Technischen Universität Dresden } \\
\text { Fetscherstraße } 74 \\
01307 \text { Dresden }\end{array}$ \\
\hline $\begin{array}{l}\text { Institut für Medizinische Mikrobiologie } \\
\text { Universitätsklinikum Medizinische Fakultät } \\
\text { Mannheim der Universität Heidelberg } \\
\text { Theodor-Kutzer-Ufer 1-3 } \\
68167 \text { Mannheim }\end{array}$ & $\begin{array}{l}\text { Institut für Medizinische Mikrobiologie } \\
\text { Westfälische Wilhelms-Universität Münster } \\
\text { Schlossplatz } 2 \\
48149 \text { Münster }\end{array}$ \\
\hline $\begin{array}{l}\text { Institut für Medizinische Mikrobiologie und } \\
\text { Hygiene } \\
\text { Klinikum der Johannes Gutenberg- } \\
\text { Universität Mainz } \\
\text { Langenbeckstraße } 1 \\
55131 \text { Mainz }\end{array}$ & $\begin{array}{l}\text { Institut für Mikrobiologie und Hygiene } \\
\text { Universitätsklinikum des Saarlandes } \\
\text { Kirrbergerstraße } \\
66421 \text { Homburg/Saar }\end{array}$ \\
\hline $\begin{array}{l}\text { Niedersächsisches Landesgesundheitsamt } \\
\text { Hannover } \\
\text { Roesebeckstraße 4-6 } \\
30449 \text { Hannover }\end{array}$ & $\begin{array}{l}\text { Institut für Medizinische Mikrobiologie } \\
\text { Universitätsklinikum Rostock } \\
\text { Schillingallee } 35 \\
18057 \text { Rostock }\end{array}$ \\
\hline $\begin{array}{l}\text { Labor Stein + Kollegen } \\
\text { Gemeinschaftspraxis für Labormedizin, } \\
\text { Mikrobiologie und Humangenetik } \\
\text { Wallstraße } 10 \\
41061 \text { Mönchengladbach }\end{array}$ & $\begin{array}{l}\text { Institut für Hygiene und } \\
\text { Laboratoriumsmedizin } \\
\text { Klinikum Krefeld } \\
\text { Lutherplatz } 40 \\
47805 \text { Krefeld }\end{array}$ \\
\hline $\begin{array}{l}\text { Institut für Laboratoriumsmedizin } \\
\text { Helios Kliniken Schwerin } \\
\text { Wismarsche Straße 393-397 } \\
19055 \text { Schwerin }\end{array}$ & $\begin{array}{l}\text { Institut für Medizinische Mikrobiologie } \\
\text { Universitätsklinikum der Otto-von-Guericke- } \\
\text { Universität Magdeburg } \\
\text { Leipziger Straße } 44 \\
39120 \text { Magdeburg }\end{array}$ \\
\hline
\end{tabular}




\begin{tabular}{|c|c|}
\hline $\begin{array}{l}\text { Medizinaluntersuchungsamt und } \\
\text { Krankenhaushygiene } \\
\text { Universitätsklinikum Schleswig-Holstein, } \\
\text { Kiel } \\
\text { Brunswiker Straße } 4 \\
24105 \text { Kiel }\end{array}$ & $\begin{array}{l}\text { Institut für Laboratoriumsmedizin } \\
\text { Städtische Kliniken Franfurt a.M.-Höchst } \\
\text { Gotenstraße 6-8 } \\
65929 \text { Frankfurt am Main }\end{array}$ \\
\hline $\begin{array}{l}\text { Landesamt für Verbraucherschutz des } \\
\text { Landes Sachsen-Anhalt } \\
\text { Wallonerberg 2-3 } \\
39104 \text { Magdeburg }\end{array}$ & $\begin{array}{l}\text { Institut für Medizinische Mikrobiologie } \\
\text { Universitätsklinikum Düsseldorf } \\
\text { Moorenstraße } 5 \\
40225 \text { Düsseldorf }\end{array}$ \\
\hline $\begin{array}{l}\text { Robert Koch-Institut } \\
\text { Nordufer } 20 \\
13353 \text { Berlin }\end{array}$ & $\begin{array}{l}\text { Institut für Medizinische Mikrobiologie der } \\
\text { Stadt Bochum } \\
\text { Medizinaluntersuchungsamt, } \\
\text { Universitätsinstitut } \\
\text { Westring } 28-30 \\
44777 \text { Bochum }\end{array}$ \\
\hline $\begin{array}{l}\text { Landesuntersuchungsanstalt für das } \\
\text { Gesundheits- und Veterinärwesen Sachsen } \\
\text { (LUA) } \\
\text { Standort Chemnitz } \\
\text { Zschopauer Straße } 87 \\
09111 \text { Chemnitz }\end{array}$ & $\begin{array}{l}\text { Institut für Medizinische Mikrobiologie und } \\
\text { Krankenhaushygiene } \\
\text { Universitätsklinikum Gießen und Marburg } \\
\text { Hans-Meerweinstraße } \\
35043 \text { Marburg }\end{array}$ \\
\hline $\begin{array}{l}\text { Klinikum Duisburg } \\
\text { Zu den Rehwiesen } 9 \\
47055 \text { Duisburg }\end{array}$ & $\begin{array}{l}\text { Thüringer Landesamt für } \\
\text { Lebensmittelsicherheit und } \\
\text { Verbraucherschutz } \\
\text { Tennstedter Straße 8-9 } \\
99947 \text { Bad Langensalza }\end{array}$ \\
\hline $\begin{array}{l}\text { Institut für Medizinische Diagnostik } \\
\text { Oderland Dr. Berthold und Kollegen } \\
\text { Am Kleistpark } 1 \\
15230 \text { Frankfurt (Oder) }\end{array}$ & $\begin{array}{l}\text { Synlab } \\
\text { Dr. med. Wimmer und Partner } \\
\text { Leitershofer Straße } 25 \\
86157 \text { Augsburg }\end{array}$ \\
\hline
\end{tabular}




\begin{tabular}{|c|c|}
\hline $\begin{array}{l}\text { Gemeinschaftspraxis Dr. med. Bernd } \\
\text { Schottdorf u.a. } \\
\text { August-Wessels-Straße } 5 \\
86154 \text { Augsburg }\end{array}$ & $\begin{array}{l}\text { Institut für Medizinische Mikrobiologie, } \\
\text { Immunologie und Hygiene } \\
\text { Uniklinik Köln } \\
\text { Goldenfelsstraße } 21 \\
50935 \text { Köln }\end{array}$ \\
\hline $\begin{array}{l}\text { Labor Dr. Wagner und Partner } \\
\text { Ärztliche Partnerschaft } \\
\text { Werner-von-Siemens-Straße 8-10 } \\
37077 \text { Göttingen }\end{array}$ & $\begin{array}{l}\text { Laboratoriumsmedizin Köln } \\
\text { Dres. med. Wisplinghoff und Kollegen } \\
\text { Classen-Kappelmann-Straße } 24 \\
50931 \text { Köln }\end{array}$ \\
\hline $\begin{array}{l}\text { Laborarztpraxis Dr. Runnebaum \& Partner } \\
\text { Wasserturmstraße } 71 \\
69214 \text { Eppelheim }\end{array}$ & $\begin{array}{l}\text { Dr. Arnold und Dr. Reinhold } \\
\text { Haugerkirchgasse } 7 \\
97070 \text { Würzburg }\end{array}$ \\
\hline $\begin{array}{l}\text { Institut für Klinische Mikrobiologie, } \\
\text { Immunologie und Hygiene } \\
\text { Universitätsklinikum Erlangen } \\
\text { Wasserturmstraße 3-5 } \\
91054 \text { Erlangen }\end{array}$ & $\begin{array}{l}\text { SRH Zentralklinikum Suhl } \\
\text { Albert-Schweitzer-Straße } 2 \\
98527 \text { Suhl }\end{array}$ \\
\hline $\begin{array}{l}\text { Gemeinschaftspraxis für } \\
\text { Laboratoriumsmedizin Hülsmann, Baczko, } \\
\text { Becker } \\
\text { Druseltalstrasse } 61 \\
34131 \text { Kassel }\end{array}$ & $\begin{array}{l}\text { Institut für Medizinische Mikrobiologie } \\
\text { Georg-August-Universität Göttingen } \\
\text { Robert-Koch-Straße } 40 \\
37075 \text { Göttingen }\end{array}$ \\
\hline
\end{tabular}




\section{$8 \quad$ Literaturverzeichnis}

Abi-Said D, Anaissie E, Uzun O, Raad I, Pinzcowski H, Vartivarian S (1997): The epidemiology of hematogenous candidiasis caused by different Candida species. Clin Infect Dis 24: 1122-8

Alexander BD, Byrne TC, Smith KL, Hanson KE, Anstrom KJ, Perfect JR, Reller LB (2007): Comparative evaluation of Etest and sensititre yeastone panels against the Clinical and Laboratory Standards Institute M27-A2 reference broth microdilution method for testing Candida susceptibility to seven antifungal agents. J Clin Microbiol 45: 698-706

Almirante B, Rodríguez D, Park BJ, Cuenca-Estrella M, Planes AM, Almela M, Mensa J, Sanchez F, Ayats J, Gimenez M (2005): Epidemiology and Predictors of Mortality in Cases of Candida Bloodstream Infection: Results from PopulationBased Surveillance, Barcelona, Spain, from 2002 to 2003. J Clin Microbiol 43: 1829-35

Anaissie EJ, Paetznick VL, Ensign LG, Espinel-Ingroff A, Galgiani JN, Hitchcock CA, LaRocco M, Patterson T, Pfaller MA, Rex JH, Rinaldi MG (1996): Microdilution antifungal susceptibility testing of Candida albicans and Cryptococcus neoformans with and without agitation: an eight-center collaborative study. Antimicrob Agents Chemother 40: 2387-91

Arendrup MV, Fuursted K, Gahrn-Hansen B, Jensen IM, Knudsen JD, Lundgren B, Schonheyder HC, Tvede M (2005): Seminational Surveillance of fungemia in Denmark: notably high rates of fungemia and numbers of isolates with reduced azole susceceptibility. J Clin Microbiol 43: 4434-40

Arikan S (2007): Current status of antifungal susceptibility testing methods. Med Mycol 45: 569-87

Arthington-Skaggs BA, Lee-Yang W, Ciblak MA, Frade JP, Brandt ME, Hajjeh RA, Harrison LH, Sofair AN, Warnock DW (2002): Comparison of visual and spectrophotometric methods of broth microdilution MIC end point determination and evaluation of a sterol quantitation method for in vitro susceptibility testing of fluconazole and itraconazole against trailing and nontrailing Candida isolates. 
Antimicrob Agents Chemother 46: 2477-81

Ásmundsdóttir LR, Erlendsdóttir H, Gottfredsson M (2002): Increasing Incidence of Candidemia: Results from a 20 -Year Nationwide Study in Iceland. J Clin Microbiol 40: 3489-92

Axner-Elings M, Botero-Kleiven S, Jensen RH, Arendrup MV (2011): Echinocandin Susceptibility Testing of Candida Isolates Collected during a 1Year Period in Sweden. J Clin Microbiol 49: 2516-21

Barchiesi F, Spreghini E, Tomassetti S, Della Vittoria A, Arzeni D, Manso E, Scalise G (2006): Effects of caspofungin against Candida guilliermondii and Candida parapsilosis. Antimicrob Agents Chemother 50: 2719-27

Barry AL, Pfaller MA, Brown SD, Espinel-Ingroff A, Ghannoum MA, Knapp C, Rennie RP, Rex JH, Rinaldi MG (2000): Quality Control Limits for Broth Microdilution Susceptibility Tests of Ten Antifungal Agents. J Clin Microbiol 38: $3457-9$

Bartizal C, Odds FC (2003): Influence of Methodological Variables on Susceptibility Testing of Caspofungin against Candida Species and Aspergillus fumigatus. Antimicrob Agents Chemother 47: 2100-7

Bennett JE, Izumikawa K, Marr KA (2004): Mechanism of Increased Fluconazole Resistance in Candida glabrata during Prophylaxis. Antimicrob Agents Chemother 48: 1773-7

Boo TW, O'Reilly B, O'Leary J, Cryan B (2005): Candidaemia in an Irish tertiary referral hospital: epidemiology and prognostic factors. Mycoses 48: 251-9

Borg-von Zepelin M, Eiffert H, Kann M, Rüchel R (1993): Changes in the spectrum of fungal isolates: results from clinical specimens gathered in 1987/88 compared with those in 1991/92 in the University Hospital Göttingen, Germany. Mycoses 36: 247-53

Borg-von Zepelin M, Kunz L, Rüchel R, Reichard U, Weig M, Gross U (2007): Epidemiology and antifungal susceptibilities of Canida spp. to six antiungal agents: results from a surveillance study on fungeamia in Germany from July 2004 to August 2005. J Antimicrob Chemother 60: 424-8 
Büchner T, Fegeler W, Bernhardt H, Brockmeyer N, Duswald KH, Herrmann M, Heuser D, Jehn U, Just-Nübling G, Karthaus M (2002): Treatment of Severe Candida Infections in High-Risk Patients in Germany: Consensus Formed by a Panel of Interdisciplinary Investigators. Eur J Clin Microbiol Infect Dis 21: 337-52

Chakrabarti A, Chander J, Kasturi P, Panigrahi D Candidaemia (1992): A 10-year study in an Indian teaching hospital. Mycoses 35: 47-51

Chapman RL (2007): Prevention and treatment of Candida infections in neonates. Semin Perinatol 31: 39-46

Chen YC, Chang SC, Sun CC, Yang LS, Hsieh WC, Luh KT (1997): Secular trends in the epidemiology of nosocomial fungal infections at a teching hospital in Taiwan, 1981 to 1993. Infect Control Hosp Epidemiol 18: 369-75

Chryssanthou E (2001): Trends in antifungal susceptibility among Swedish Candida species bloodstream isolates from 1994 to 1998: comparison E-test and the Sensititre YeastOne Colometric Antifungal Panel with NCCLS 27-A2 reference method. J Clin Microbiol 39: 4181-3

Chryssanthou E, Cuenca-Estrella M (2002): Comparison of the Antifungal Susceptibility Testing Subcommittee of the European Committee on Antibiotic Susceptibility Testing Proposed Standard and the E-Test with NCCLS Broth Microdilution Method for Voriconazole and Caspofungin Susceptibility Testing of Yeast Species. J Clin Microbiol 10: 3841-4

CLSI M27-A3: Reference Method for Broth Dilution Antifungal Susceptibility Testing of Yeasts: Approved Standard M27-A Third Edition. Clinical and Laboratory Standards Institute, Wayne/PA, USA 2008

Coleman DC, Rinaldi MG, Haynes KA, Rex JH, Summerbell RC, Anaissie EJ, Li A, Sullivan DJ (1998): Importance of Candida species other than Candida albicans as opportunistic pathogens. Med Mycol 36: 156-65

Colombo AL, Barchiesi F, McGough DA, Rinaldi MG (1995): Comparison of Etest and National Committee for Clinical Laboratory Standards broth macrodilution method for azole antifungal susceptibility testing. J Clin Microbiol 33: 535-40 
Colombo AL, Nucci M, Salomao R, Branchini ML, Richtmann R, Derossi A, Wey SB (1999): High rate of non-albicans candidemia in Brazilian tertiary care hospitals. Diagn Microbiol Infect Dis 34: 281-6

Cormican MG, Pfaller MA (1996): Standardization of antifungal susceptibility testing. J Antimicrob Chemother 38: 561-78

Cuenca-Estrella M, Díaz-Guerra TM, Mellado E, Rodriguez-Tudela JL (2001): Flucytosine Primary Resistance in Candida Species an Cryptococus neoformans. Eur J Clin Microbiol Infect Dis 20: 276-9

Cuenca-Estrella M, Rodriguez D, Almirante B, Morgan J, Planes AM, Almela M, Mensa J, Sanchez F, Ayats J, Gimenez M (2005): In vitro susceptibilities of bloodstream isolates of Candida species to six antifungal agents: results from a population-based active surveillance programme, Barcelona, Spain, 2002-2003 J Antimicrob Chemother 55: 194-9

Davey KG, Szekely A, Johnson EM, Warnock DW (1998): Comparison of a new commercial colorimetric microdilution method with a standart method for in-vitro susceptibility testing of Candida spp. and Cryptococcus neoformans. J Antimicrob Chemother 42: 439-44

Diekema DJ, Messer SA, Brueggemann AB, Coffman SL, Doern GV, Herwaldt LA, Pfaller MA (2002): Epidemiology of Candidemia: 3-Year Results from the Emerging Infections and the Epidemiology of lowa Organisms Study. J Clin Microbiol 40: 1298-302

Diekema DJ, Messer SA, Hollis RJ, Boyken L, Tendolkar S, Kroeger J, Jones RN, Pfaller MA (2009): A global evaluation of voriconazole activity tested against recent clinical isolates of Candida spp. Diagn Microbiol Infect Dis 63: 233-6

Douglas CM, D'Ippolito JA, Shei GJ, Meinz M, Onishi J, Marrinan JA, Li W, Abruzzo GK, Flattery A, Bartizal K (1997): Identification of the FKS1 gene of Candida albicans as the essential target of 1,3-beta-D-glucan synthase inhibitors. Antimicrob Agents Chemother 41: 2471-9

Edmond MB, Wallace SE, McClish DK, Pfaller MA, Jones RN, Wenzel RP (1999): Nosocomial Bloodstream Infections in United States Hospitals: A ThreeYear Analysis. Clin Infect Dis 29: 239-44 
Eggimann P, Garbino J, Pittet D (2003): Epidemiology of Candida species infections in critically ill non-immunosuppressed patients. Lancet Infect Dis 3: 685-702

Eloy O, Blanc V, Pina P, Gaudart A, Bressolle ML, Plainvert C, Decousser JW, Pangon $\mathrm{B}$, Allouch $\mathrm{PY}$; le Collège de bactériologie virologie hygiène (ColBVH) (2006): Epidemiology of candidemia: results of a one month French hospitalsbased surveillance study in 2004. Pathol Biol (Paris) 54: 523-30

Engel C, Brunkhorst FM, Bone HG, Bunkhorst R, Gerlach H, Gromd S, Gruendling M, Huhle G, Jaschinski U, John S (2007): Epidemiology of sepsis in Germany: results from a national prospective multicenter study. Intensive Care Med 33: 606-18

Espinel-Ingroff A, Pfaller M, Erwin ME, Jones RN (1996): Interlaboratory evaluation of method for testing antifungal susceptibilities of pathogenic yeasts to five antifungal agents by using Casitone agar and solidified RPMI 1640 medium with 2\% glucose. J Clin Microbiol 34: 848-52

Etest Arbeitsanleitung 3b, AB Biodisk, Solna, Schweden 2000

Fleck R, Dietz A, Hof H (2007): In vitro susceptibility of Candida species to five antifungal agents in a German university hospital assessed by the reference broth microdilution method and Etest. J Antimicrob Chemother 59: 767-71

Garbino J, Kolarova L, Rohner P, Lew D, Pichna P, Pittet D (2002): Secular Trends of Candidemia Over 12 Years in Adult Patients at a Tertiary Care Hospital. Medicine 81: 425-33

Garcia-Effron G, Kontoyiannis DP, Lewis RE, Perlin DS (2008): Caspofunginresistant Candida tropicalis strains causing breakthrough fungemia in patients at high risk for hematologic malignancies. Antimicrob Agents Chemother 52: 4181-3

Geffers C, Zuschneid I, Sohr D, Rüden H, Gastmeier (2004): Microbiological Isolates Associated with Nosocomial Infections in Intensive Care Units: Data of 274 Intensive Care Units Participating in the German Nosocomial Infections Surveillance System (KISS). Anästhesiol Intensivmed Notfallmed Schmerzther 39: $15-9$

Georgeopapadakou NH, Walsh TJ (1996): Antifungal Agents: Chemotherapeutic Targets and Immunologic Strategies. Antimicrob Agents Chemother 40: 279-91 
Ghannoum MA, Ibrahim AS, Fu Y, Shafiq MC, Edwards JE Jr, Criddle RS (1992): Susceptibility testing of Cryptococcus neoformans: a microdilution technique. $J$ Clin Microbiol 30: 2881-6

Glasmacher A, Molitor E, Hahn C, Bomba K, Ewig S, Leutner C, Wardelmann E, Schmidt-Wolf IG, Mezger J, Marklein G, Sauerbruch T (1998): Antifungal prophylaxis with itraconazole in neutropenic patients with acute leukaemia. Leukemia 12: 1338-43

Glasmacher A, Hahn C, Molitor E, Marklein G, Sauerbruch T, Schmidt-Wolf IG (1999): Itraconazole through concentrations in antifungal prophylaxis with six different dosing regimes using hydroxypopyl-beta-cyclodextrin oral solution or coated-pellet capsules. Mycoses 42: 591-600

Godoy P, Tiraboschi IN, Severo LC, Bustamante B, Calvo B, Almeida LP, da Matta DA, Colombo AL (2003): Species distribution and antifungal susceptibility profile of Candida spp. bloodstream isolates from Latin American hospitals. Mem Inst Oswaldo Cruz 98: 401-5

Guinea J, Sanchez-Somolinos M, Cueavas O, Pelaez T, Bouza E (2006): Fluconazole resistance mechanisms in candida krusei: the contribution of effluxpumps. Med Mycol 44: 575-8

Guinea J, Recio S, Escribano P, Torres-Narbona M, Peláez T, Sánchez-Carillo C, Rodríguez-Créixems M, Bouza E (2010): Rapid Antifungal Susceptibility Determination for Yeast Isolates by Use of Etest Directly on Blood Samples from Patients with Fungemia. J Clin Microbiol 48: 2205-12

Hajjeh RA, Sofair AN, Harrison LH, Lyon GM, Arthington-Skaggs BA, Mirza SA, Phelan M, Morgan J, Lee-Yang W, Ciblak MA (2004): Incidence of Bloodstream Infections Due to Candida Species and in Vitro Susceptibilities of Isolates Collected from 1998 - 2000 in a Population-Based Active Surveillance Program. J Clin Microbiol 42: 1519-27

Hoban DJ, Zhanel GG, Karlowsky JA (1999): In vitro susceptibilities of Candida and Cryptococcus neoformans isolates from blood cultures of neutropenic patients. Antimicrob Agents Chemother 43: 1463-4 
Hof $H$ (2008): Developments in the epidemiology of invasive fungal infections implications for the empiric and targeted antifungal therapy. Mycoses 51: 1-6

Kao AS, Brandt ME, Pruitt WR, Conn LA, Perkins BA, Stephens DS, Baughman WS, Reingold AL, Rothrock G, Pfaller MA (1999): The Epidemiology of Candidemia in Two United States Cities: Results of a Population-Based Aktive Surveillance. Clin Infect Dis 29: 1164-70

Kibbler CC, Seaton S, Barnes RA, Gransden WR, Holliman RE, Johnson EM, Perry JD, Sullivan DJ, Wilson JA (2003): Management and outcome of bloodstream infections due to Candida species in England and Wales. J Hosp Infect 54: 18-24

Klingspor L, Tornqvist E, Johansson A, Petrini B, Forsum U, Hedin G (2004): A prospective epidemiological survey of candidaemia in Sweden. Scand J Infect Dis 36: $52-5$

Knoke M, Schulz K, Bernhardt H (1997): Dynamics of Candida isolations from humans from 1992-1995 in Greifswald, Germany. Mycoses 40: 105-10

Koç AN, Gökahmetòğlu S, Oğuzkaya M (2000): Comparison of Etest with the broth microdilution method in susceptibility testing of yeast isolates against four antifungals. Mycoses 43: 293-7

Kumm K: Candida-Blutkulturisolate in Deutschland und Österreich. Spektrum, Klinik und Empfindlichkeit gegenüber sechs ausgewählten Antimykotika. Med. Diss. Göttingen 2008

Krcmery V, Barnes AJ (2002): Non-albicans Candida spp. causing fungaeamia: pathogenicity and antifungal resistance. J Hosp Infect 50: 243-60

Lamping E, Ranchod A, Nakamura K, Tyndall JD, Niimi K, Holmes AR, Niimi M, Cannon RD (2009): Abc1p is a multidrug efllux transporter that tips the balance in favor of innate azole resistance in Candida krusei. Antimicrob Agents Chemother 53: $354-69$

Laupland KB, Gregson DB, Church DL, Ross T, Elsayed S (2005): Invasive Candida species infections: a 5 year populationbased assessment. J Antimicrob Chemother 56: 532-7 
Laverdiere M, Restieri C, Habel F (2002): Evaluation of the in vitro activity of caspofungin against bloodstream isolates of Candida species from cancer patients: comparison of Etest and NCCLS reference methods. Int J Antimicrob Agents 20: 468-71

Levy I, Rubin LG, Vashishtha S, Tucci V, Sood SK (1998): Emergence of Candida parapsilosis as the predominant species causing candidemia in children. Clin Infect Dis 26: 1086-8

Lozano-Chiu M, Arikan S, Paetznick VL, Anaissie EJ, Rex JH (1999): Optimizing voriconazole susceptibility testing of Candida: effects of incubation time, endpoint rule, species of Candida, and level of fluconazole susceptibility. J Clin Micobiol 37: $2755-9$

Luzzati R, Amalfitano G, Lazzarini L, Soldani F, Bellino S, Solbiati M, Danzi MC, Vento S, Todeschini G, Vivenza C (2000): Nosocomial candidemia in nonneutropenic patients at an Italian tertiary care hospital. Eur J Clin Microbiol Infect Dis 19: $602-7$

Lyytikäinen O, Lumio J, Sarkkinen H, Kolho E, Kostiala A, Ruutu P, Hospital Infection Surveillance Team (2002): Nosocomial Bloodstream Infections in Finnish Hospitals during 1999-2000. Clin Infect Dis 35: e14-9

Marchetti O, Bille J, Fluckiger U, Eggimann P, Ruef C, Garbino J, Calandra T, Glauser MP, Täuber MG, Pittet D (2004): Epidemiology of Candidemia in Swiss Tertiary Care Hospitals: Secular Trends, 1991-2000. Clin Infect Dis 38: 311-20

Martin D, Persat F, Piens MA, Picot S (2005): Candida species distribution in bloodstream cultures in Lyon, France, 1998-2001. Eur J Clin Microbiol Infect Dis 24: 329-33

Matar MJ, Ostrosky-Zeichner L, Paetznick VL, Rodriguez JR, Chen E, Rex JH (2003): Correlation between E-Test, Disk Diffusion and Microdilution Methods for Antifungal Susceptibility Testing of Fluconazole and Voriconazole. Antimicrob Agents Chemother 47: 1647-51

Maxwell MJ, Messer SA, Hollis RJ, Boyken L, Tendolkar S, Diekema DJ, Pfaller MA (2003): Evaluation of Etest method for determining fluconazole and voriconazole MICs for 279 clinical isolates of Candida species infrequently isolated from blood J Clin Microbiol 41: 1087-90 
McMullan R, McClurg R, Xu J, Moore JE, Millar BC, Crowe M, Hedderwick S (2002): Trends in the Epidemiology of Candida Bloodstream Infections in Northern Ireland between January 1984 and December 2000. J Infect 45: 25-8

Metin DY, Hilmioglu-Polat S, Samlioglu P, Doganay-Oflazoglu B, Inci R, Tumbay E (2011): Evaluation of Antifungal Susceptibility Testing with Microdilution and Etest Methods of Candida Blood Isolates. Mycopathologia 172: 187-99

Morace G, Amato G, Bistoni F, Fadda G, Marone P, Montagna MT, Oliveri S, Polonelli L, Rigoli R, Mancuso I (2002): Multicenter comparative evaluation of six commercial systems and the national committee for clinical laboratory standards m27-a broth microdilution method for fluconazole susceptibility testing of Candida species. J Clin Microbiol 40: 2953-8

NCCLS M27-A2: Reference Method for Broth Dilution Antifungal Susceptibility Testing of Yeasts: Approved Standard M27-A Second Edition. National Committee for Clinical Laboratory Standards, Wayne/PA, USA 2002

Negri M, Henriques M, Svidzinski TI, Paula CR, Oliveira R (2009): Correlation between Etest, disk diffusion, and microdilution methods for antifungal susceptibility testing of Candida species from infection and colonization. J Clin Lab Anal 23: 324-30

Nguyen MH, Peacock JE Jr, Morris AJ, Tanner DC, Nguyen ML, Snydman DR, Wagener MM, Rinaldi MG, Yu VL (1996): The changing face of candidemia: Emergence of non-Candida albicans species and antifungal resistance. Am J Med 100: 617-23

Nguyen MH, Clancy CJ, Yu VL, Yu YC, Morris AJ, Snydman DR, Sutton DA, Rinaldi MG (1998): Do in vitro susceptibility data predict the microbiologic response to amphotericin $b$ ? Results of a prospective study of patients with Candida fungemia. J Infect Dis 177: 425-30

Odds FC, Brown AJ, Gow NA (2003): Antifungal agents: mechanisms of action. Trends Microbiol 11: 272-9

Odds FC, Motyl M, Andrade R, Bille J, Cantón E, Cuenca-Estrella M, Davidson A, Durussel C, Ellis D, Foraker E (2004): Interlaboratory Comparison of Results of Susceptibility Testing with Caspofungin against Candida and Aspergillus Species. 
J Clin Microbiol 42: 3475-82

Odds FC, Hanson MF, Davidson AD, Jacobsen MD, Wright P, Whyte JA, Gow NA, Jones BL (2007): One year prospective survey of Candida bloodstream infections in Scotland. J Med Microbiol 56: 1066-75

Oxman DA, Chow JK, Frendl G, Hadley S, Hershkovitz S, Ireland P, McDermott LA, Tsai K, Marty FM, Kontoyiannis DP, Golan Y (2010): Candidaemia associated with decreased in vitro fluconazole susceptibility: is Candida speciation predictive of the susceptibility pattern? J Antimicrob Chemother 65: 1460-5

Park BJ, Arthington-Skaggs BA, Hajjeh RA, lqbal N, Ciblak MA, Lee-Yang W, Hairston MD, Phelan M, Plikaytis BD, Sofair AN, Harrison LH, Fridkin SK, Warnock DW (2006): Evaluation of amphotericin B interpretive breakpoints for Candida bloodstream isolates by correlation with therapeutic outcome. Antimicrob Agents Chemother 50: 1287-92

Park JY, shin JH, Uh Y, Kim EC, Kee SJ, Kim SH, Shin MG, Suh SP, Ryang DW (2008): In vitro amphotericin B susceptibility of korean bloodstream yeast isolates assessed by the CLSI broth microdilution method, Etest, and Minimum fungicidal concentration test. Korean J Lab Med 28: 346-52

Pfaller MA, Diekema DJ (2002): Role of Sentinel Surveillance of Candidemia: Trends in Species Distribution and Antifungal Susceptibility. J Clin Microbiol 40: 3551-7

Pfaller MA, Diekema DJ (2007): Epidemiology of invasive candidiasis: a persistent public health problem. Clin Microbiol Rev 20: 133-63

Pfaller MA, Messer SA, Hollis RJ (1994): Strain delineation and antifungal susceptibilities of epidemiologically related and unrelated isolates of Candida lusitaniae. Diagn Microbiol Infect Dis 20: 127-33

Pfaller MA, Messer SA, Bolmström A, Odds FC, Rex JH (1996): Multisite reproducibility of the Etest MIC method for antifungal susceptibility testing of yeast isolates. J Clin Microbiol 34: 1691-3

Pfaller MA, Jones RN, Messer SA, Edmond MB, Wenzel RP (1998a): National Surveillance of Nosocomial Blood Stream Infection Due to Species of Candida other than Candida albicans: Frequency of Occurrence and Antifungal 
Susceptibility in the SCOPE Program. Diagn Microbiol Infect Dis 30: 121-9

Pfaller MA, Jones RN, Doern GV, Sader HS, Hollis RJ, Messer SA for the Sentry Participant Group (1998b): International Surveillance of Bloodstream Infections Due to Candida Species: Frequency of Occurence and Antifungal Susceptibilities of Isolates Collected in 1997 in the United States, Canada, and South America for the SENTRY Program. J Clin Microbiol 36: 1886-89

Pfaller MA, Messer SA, Hollis RJ, Jones RN, Doern GV, Brandt ME, Hajjeh RA (1999): Trends in Species Distribution and Susceptibility to Fluconazole among Blood Stream Isolates of Candida Species in the United States. Diagn Microbiol Infect Dis 33: 217-22

Pfaller MA, Messer SA, Houston A, Mills K, Bolmstrom A, Jones RN (2000): Evaluation of the Etest method for determining voriconazole susceptibilities of 312 clinical isolates of Candida species by using three different agar media. $J$ Clin Microbiol 38: 3715-7

Pfaller MA, Diekema DJ, Jones RN, Sader HS, Fluit AC, Hollis RJ, Messer SA, The Sentry Participant Group (2001): International Surveillance of Bloodstream Infections Due to Candida Species: Frequency of Occurrence and In Vitro Susceptibilities to Fluconazol, Ravuconazole, and Voriconazole of Isolates Collected from 1997 through 1999 in the SENTRY Antimicrobial Surveillance Program. J Clin Microbiol 39: 3254-9

Pfaller MA, Messer SA, Boyken L, Huynh RJ, Hollis RJ, Diekema DJ (2002): In Vitro Activities of 5-Fluorocytosine against 8,803 Clinical isolates of Candida spp.: Global Assessment of Primary Resistance Using National Committee for Clinical Laboratory Standards Susceptibility Testing Methods. Antimicrob Agents Chemother 46: 3518-21

Pfaller MA, Diekema DJ, Boyken L, Messer SA, Tendolkar S, Hollis RJ (2003a): Evaluation of the Etest and Disk Diffusion Methods for Determining Susceptibilities of 235 Bloodstream Isolates of Candida glabrata to Fluconazole and Voriconazole. J Clin Microbiol 41: 1875-80

Pfaller MA, Diekema DJ, Messer SA, Hollis RJ, Jones RN (2003b): In Vitro Activities of Caspofungin Compared with Those of Fluconazole and Itraconazole against 3,959 Clinical Isolates of Candida spp., Including 157 Fluconazole- 
Resitant Isolates. Antimicrob Agents Chemother 47: 1068-71

Pfaller MA, Diekema DJ, Rex JH, Espinel-Ingroff A, Johnson EM, Andes D, Chaturvedi V, Ghannoum M, Odds FC, Rinaldi MG (2006a): Correlation of MIC with Outcome for Candida Species Tested against Voriconazole: Analysis and Proposal for Interpretive Breakpoints. J Clin Microbiol 44: 819-26

Pfaller MA, Boyken L, Hollis RJ, Messer SA, Tendolkar S, Diekema DJ (2006b): Global surveillance of in vitro activity of micafungin against Candida: a comparison with caspofungin by CLSI-recommended methods. J Clin Microbiol 44: $3533-8$

Pfaller MA, Diekema DJ, Sheehan DJ (2006c): Interpretive Beakpoints for Fluconazole and Candida Revisited: a Blueprint for the Future of Antifungal Susceptibility Testing. Clin Microbiol Rev 19: 435-47

Pfaller MA, Diekema DJ, Ostrosky-Zeichner L, Rex JH, Alexander BD, Andes D, Brown SD, Chaturvedi V, Ghannoum MA, Knapp CC (2008a): Correlation of MIC with outcome for Candida species tested against Caspofungin, anidulafungin, and micafungin: analysis and proposal for interpretive MIC breakpoints. J Clin Microbiol 46: 2620-9

Pfaller MA, Boyken L, Hollis RJ, Kroeger J, Messer SA, Tendolkar S, Diekema DJ (2008b): In Vitro Susceptibility of Invasive Isolates of Candida spp. to Anidulafungin, Caspofungin, and Micafungin: Six Years of Global Surveillance. J Clin Microbiol 46: 150-6

Pfaller MA, Diekema DJ, Gibbs DL, Newell VA, Ellis D, Tullio V, Rodloff A, Fu W, Ling TA and the Global Antifungal Surveillance Group (2010): Results from ARTEMIS DISK Global Antifungal Surveillance Study, 1997 to 2007: a 10.5-Year Analysis of Susceptibilities of Candida Species to Fluconazole and Voriconazole as Determined by CLSI Standardized Disk Diffusion. J Clin Microbiol 48: 1366-77

Pfaller MA, Diekema DJ, Andes D, Arendrup MC, Brown SD, Lockhart SR, Motyl M, Perlin DS, the CLSI Subcommittee for Antifungal Testing (2011a): Clinical breakpoints for the echinocandins and Candida revisited: Integration of molecular, clinical, and microbiological data to arrive at species-specific interpretive criteria. Drug Resist Updat 14: 164-76 
Pfaller MA, Castanheira M, Messer SA, Moet GJ, Jones RN (2011b): Echinocandin and triazole antifungal susceptibility profiles for Candida spp., Cryptococcus neoformans, and Aspergillus fumigatus: cutoff values to characterize resistance in the SENTRY Antimicrobial Surveillance Program (2009). Diagn Microbiol Infect Dis 69: 45-50

Pittet D, Li N, Woolson RF, Wenzel RP (1997): Microbiological factors influencing the outcome of nosocomial bloodstream infections: a 6-year validated, population-based model. Clin Infect Dis 24: 1068-78

Poikonen E, Lyytikäinen O, Anttila VJ, Ruutu P (2003): Candidemia in Finland, 1995-1999. Emerg Infect Dis 9: 985-90

Posteraro B, Romano L, Sanguinetti M, Masucci L, Morace G, Fadda G (2000): Commercial systems for fluconazole susceptibility testing of yeasts: comparison with the broth microdilution method. Diagn Microbiol Infect Dis. 38: 29-36

Rangel-Frausto MS, Wiblin T, Blumberg HM, Saiman L, Patterson J, Rinaldi M, Pfaller MA, Edwards Jr JE, Jarvis W, Dawson J (1999): National Epidemiology of Mycoses Survey (NEMIS): Variations in Rates of Bloodstream Infections Due to Candida Species in Seven Surgical Intensive Care Units and Six Neonatal Intensive Care Units. Clin Infect Dis 29: 253-58

Revankar SG, Kirkpatrick WR, McAtee RK, Fothergill AW, Redding SW, Rinaldi MG, Patterson TF (1998): Interpretation of trailing endpoints in antifungal susceptibility testing by the National Committee for Clinical Laboratory Standards method. J Clin Microbiol 36: 153-6

Rex JH, Pfaller MA, Rinaldi MG, Polak A, Galgiani JN (1993): Antifungal Susceptibility Testing. Clin Microbiol Rev 6: 367-81

Rex JH, Cooper CR Jr, Merz WG, Galgiani JN, Anaissie EJ (1995): Detection of amphotericin B-resistant Candida isolates in a broth-based system. Antimicrob Agents Chemother 39: 906-9

Rex JH, Pfaller MA, Galgiani JN, Bartlett MS, Espinel-Ingroff A, Ghannoum MA, Lancaster M, Odds FC, Rinaldi MG, Walsh TJ (1997): Development of Interpretive Breakpoints for Antifungal Susceptibility Testing: Conceptual Framework and Analysis of In Vitro-In Vivo Correlation Data for Fuconazole, Itraconazole and Candida Infections. Clin Infect Dis 24: 235-47 
Rex JH, Walsh TJ, Sobel JD, Filler SG, Pappas PG, Dismukes WE, Edwards JE (2000): Practice guidelines for the treatment of candidiasis. Clin Infect Dis 30: $662-78$

Rex JH, Pfaller MA, Walsh TJ, Chaturvedi V, Espinel-Ingroff A, Ghannoum MA, Gosey LL, Odds FC, Rinaldi MG, Sheehan DJ (2001): Antifungal Susceptibility Testing: Practical Aspects and Current Challenges. Clin Microbiol Rev 14: 643-58

Richet H, Roux P, Des Champs C, Esnault Y, Andremont A (2002): Candidemia in French hospitals: incidence rates and characteristics. Clin Microbiol Infect 8: $405-12$

Roilides E, Farmaki E, Evdoridou J, Dotis J, Hatziioannidis E, Tsivitanidou M, Bibashi E, Filioti I, Sofianou D, Gil-Lamaignere C (2004): Neonatal candidiasis: analysis of epidemiology, drug susceptibility, and molecular typing of causative isolates. Eur J Clin Microbiol Infect Dis 23: 745-50

Rüchel R, Kellner S, Schaffrinski M (2002): Renewed increase in Candida albicans among yeast isolates from the Göttingen university hospital. Mycoses 45: $109-10$

Sandven P (2000): Epidemiology of Candidemia. Rev Iberoam Micol 17: 73-81

Sandven P, Nilsen K, Digranes A, Tjade T, Lassen J (1997): Candida norvegensis: a fluconazole-resistant species. Antimicrob Agents Chemother 41: $1375-6$

Sandven P, Bevanger L, Digranes A, Gaustad P, Haukland HH, Steinbakk M (1998): Constant low rate of fungemia in norway, 1991 to 1996. The Norwegian Yeast Studay Group. J Clin Microbiol 36: 3455-9

Sanglard D, Odds FC (2002): Resistance of Candida species to antifungal agents: molecular mechanisms and clinical consequences. Lancet Infect Dis 2: 73-85

Schmalreck AF, Fegeler W (1996): Criteria for a microdilution susceptibility testing method of fluconazole: proposal of a standardized testing method for yeasts. Mycoses 39: 12-6

Schmalreck AF, Kottmann I, Reiser A, Ruffer U, Vanca E (1996): Susceptibility testing of yeasts against fluconazole: comparison of the Etest method with 
microdilution and agar dilution. Mycoses 39: 31-8

Schwesinger G, Junghans D, Schröder G, Bernhardt H, Knoke M (2005):

Candidosis and aspergillosis as autopsy findings from 1994 to 2003. Mycoses 48: $176-80$

Sewell DL, Pfaller MA, Barry AL (1994): Comparison of broth macrodilution, broth microdilution, and $E$ test antifungal susceptibility tests for fluconazole. J Clin Microbiol 32: 2099-102

Tortorano AM, Rigoni AL, Biraghi E, Prigitano A, Viviani MA (2003): The European Confederation of Medical Mycology (ECMM) survey of candidaemia in Italy: antifungal susceptibility patterns of 261 non-albicans Candida isolates from blood. J Antimicrob Chemother 52: 679-82

Tortorano AM, Peman J, Bernhardt H, Klingspor L, Kibbler CC, Faure O, Biraghi E, Canton E, Zimmermann K, Seaton S (2004): Epidemiology of Candidaemia in Europe: Results of 28-Month European Confederation of Medical Mycology (ECMM) Hospital-Based Surveillance Study. Eur J Clin Microbiol Infect Dis 23: $317-22$

Tortorano AM, Kibbler C, Peman J, Bernhardt H, Klingspor L, Grillot R (2006): Candidaemia in Europe: Epidemiology and Resistance. Int $\mathrm{J}$ Antimicrob Agents 27: $359-66$

Trick WE, Fridkin JR, Edwards JR, Hajjeh RA, Gaynes RP, National Nosocomial Infections Surveillance System Hospitals (2002): Secular Trend of HospitalAcquired Candidemia among Intensive Care Unit Patients in the United States during 1989-1999. Clin Infect Dis 35: 627-30

Viscoli C, Girmenia C, Marinus A, Collette L, Martino P, Vadercam B, Doyen C, Lebeau B, Spence D, Krcmery V (1999): Candidemia in cancer patients: a prospective, multicenter surveillance study by the Invasive Fungal Infection Group (IFIG) of the European Organization for Research and Treatment of Cancer (EORTC). Clin Infect Dis 28: 1071-9

Viviani MA (1995): Flucytosine - what is its future? J Antimicrob Chemother 35: 241-4

Wanger A, Mills K, Nelson PW, Rex JH (1995): Comparison of Etest and National Committee for Clinical Laboratory Standards broth macrodilution method for 
antifungal susceptibility testing: enhanced ability to detect amphotericin Bresistant Candida isolates. Antimicrob Agents Chemother 39: 2520-2

Warnock DW, Johnson EM, Rogers TR (1998): Multi-centre evaluation of the Etest method for antifungal drug susceptibility testing of Candida spp. and Cryptococcus neoformans. J Antimicrob Chemother 42: 321-31

Wenzel RP, Edmond MB (2001): The Impact of Hospital-Acquired Bloodstream Infections. Emerg Infect Dis 7: 174-7

Wey SB, Mori M, Pfaller MA, Woolson RF, Wenzel RP (1988): Hospital-Acquired Candidemia: the Attributable Mortality and Excess Length of Stay. Arch Intern Med 148: $2642-5$

Yapar N, Uysal U, Yucesoy M, Cakir N, Yuce A (2006): Nosocomial bloodsteam infections associated with Candida Species in a Turkish University Hospital. Mycoses 49: 134-8 


\section{Danksagung}

Mein Dank gilt Prof. Dr. Margarete Borg-von Zepelin für die Vergabe des Dissertationsthemas sowie die persönliche, umfassende und anhaltende Betreuung.

Ich danke Kerstin für die bereichernde Zusammenarbeit und gegenseitige Unterstützung. 